\title{
Material Metodológico \\ Guía de práctica clínica sobre glaucoma de ángulo abierto
}

GUÍAS DE PRÁCTICA CLÍNICA EN EL SNS

MINISTERIO DE SANIDAD, SERVICIOS SOCIALES E IGUALDAD 


\section{Índice}

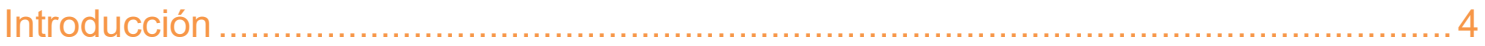

1. Clasificación de la calidad de la evidencia y graduación de la fuerza de las

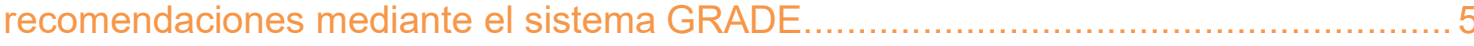

1.1.Elaboración de las preguntas y definición de los desenlaces de interés .............5 5

1.2.Clasificación de la calidad de la evidencia .................................................. 5

1.3.Graduación de la fuerza de las recomendaciones ............................................... 7

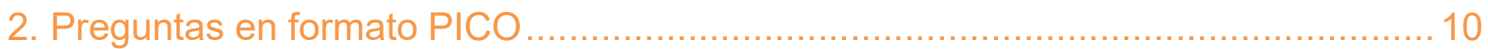

2.1. Factores de riesgo, despistaje y pruebas mínimas para el diagnóstico del

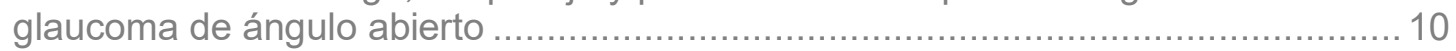

2.2. Tratamiento del glaucoma de ángulo abierto .............................................. 11

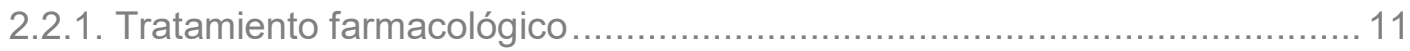

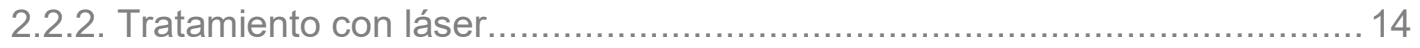

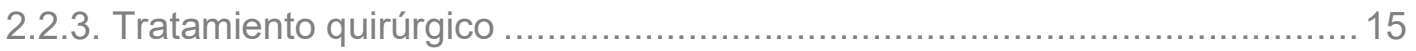

2.3. Anestesia en cirugía penetrante y no penetrante de glaucoma ....................... 18

2.4. Control evolutivo del paciente adulto con GAA ............................................. 19

3. Estrategias de búsqueda .............................................................................. 20

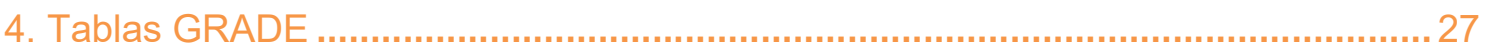

4.1.1 Factores de riesgo para el desarrollo del glaucoma de ángulo abierto ........ 27

4.1.2 Pruebas de cribado del glaucoma de ángulo abierto................................ 34

4.2.Tratamiento farmacológico del glaucoma de ángulo abierto .............................55

4.2.1. Opciones farmacológicas en el tratamiento del glaucoma de ángulo abierto

4.2.1.1. Parasimpaticomiméticos frente betabloqueadores............................. 55

4.2.1.2. Inhibidores de la anhidrasa carbónica frente a betabloqueadores ......56 56

4.2.1.3. Análogos de las prostaglandinas frente a betabloqueadores ..............57

4.2.1.4. Análogos de prostaglandinas frente a inhibidor de la anhidrasa

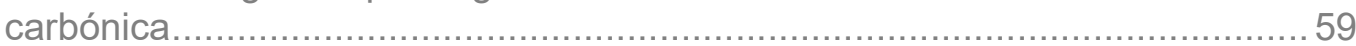

4.2.1.5. Betabloqueadores frente a agonistas alfa selectivos ....................... 61

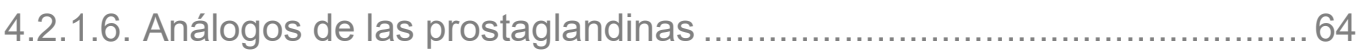

4.2.1.7. Análogos de prostaglandinas frente a agonista alfa selectivos ...........68

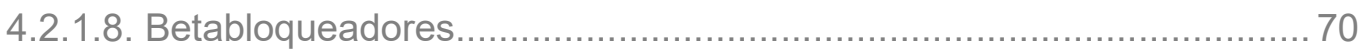

4.2.2. Uso de combinaciones fijas de fármacos ................................................. 73

4.2.3. Uso de fármacos libres de preservantes ................................................ 75

4.3. Tratamiento con láser del glaucoma de ángulo abierto................................... 80 
4.3.1. Uso de diferentes tipos de láser en el tratamiento del glaucoma de ángulo abierto

4.3.1.1. Uso de láser diodo respecto al láser argón ...................................... 80

4.3.1.2. Uso de láser selectivo respecto al láser argon ................................. 86

4.3.2. Trabeculoplastia láser respecto al tratamiento farmacológico en el glaucoma de ángulo abierto.

4.3.3. Trabeculoplastia con láser asociada al tratamiento farmacológico respecto al tratamiento farmacológico en el glaucoma de ángulo abierto.

4.3.4. Trabeculoplastia con láser frente trabeculectomía en el tratamiento del

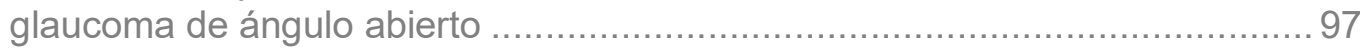

4.4 Tratamiento quirúrgico en el glaucoma de ángulo abierto ............................... 98

4.4.1 Trabeculectomía en el tratamiento del glaucoma de ángulo abierto............98

4.4.1.1.Trabeculectomía respecto al tratamiento farmacológico ..................... 98

4.4.1.2. Trabeculectomía asociada a antimetabolitos en comparación a trabeculectomía sola ....................................................................... 101

4.4.2. Cirugía no penetrante en el tratamiento del glaucoma de ángulo abierto 108

4.4.2.1. Cirugía no penetrante respecto a la trabeculectomía....................... 108

4.4.2.2.Uso de antimetabolitos en la cirugía no penetrante ......................... 119

4.4.2.3. Uso de implantes en la cirugía no penetrante ................................. 123

4.4.3 Uso de otras técnicas quirúrgicas/dispositivos en el tratamiento del glaucoma

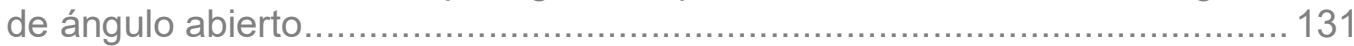

4.4.3.1 Uso del implante Ex-PRESS en el tratamiento del glaucoma de ángulo abierto

4.4.3.2. Uso de dispositivos de drenaje (valvulados o no) en el tratamiento del glaucoma de ángulo

4.4.3.3. Uso de la microtrabeculectomía en el tratamiento del glaucoma de ángulo abierto

4.5. Anestesia locoregional en la cirugía del glaucoma de ángulo abierto .............. 143

4.5.1. Anestesia tópica respecto a anestesia retrobulbar. ...............................143

4.5.2. Anestesia tópica respecto a anestesia subtenoniana .............................. 149

4.5.3. Anestesia tópica respecto a anestesia peribulbar ................................... 152

4.5.4. Anestesia de contacto vs. otras tecnicas anestésicas ............................. 154

4.5.5. Anestesia subtenoniana vs. anestesia retrobulbar ................................. 160 


\section{Introducción}

En este documento se describen de forma metodológica las etapas y procedimientos utilizados para elaborar la Guía de práctica clínica sobre glaucoma de ángulo abierto. Para ello, se han utilizado los principios metodológicos del programa de Guías de Práctica Clínica (GPC) en el Sistema Nacional de Salud, recogidos en el manual metodológico de elaboración de GPC de GuiaSalud.

En el caso de esta guía, se ha optado por utilizar el sistema GRADE (Grading of Recommendations Assessment, Development and Evaluation) para sintetizar y evaluar la evidencia a cada pregunta clínica, así como para formular las recomendaciones. 


\section{Clasificación de la calidad de la evidencia y graduación de la fuerza de las recomendaciones mediante el sistema GRADE}

Para clasificar la calidad de la evidencia y graduar la fuerza de las recomendaciones de esta Guía de Práctica Clínica (GPC), y según el manual metodológico, se han seguido las directrices del grupo de trabajo GRADE (Grading of Recommendations of AssessmentDevelopment and Evaluation) (1-3). Las principales etapas que conforman el sistema GRADE son las que se detallan en los siguientes subapartados.

\subsection{Elaboración de las preguntas y definición de los desenlaces de interés}

Las preguntas clínicas suelen estructurarse en forma de PICO, es decir, incorporan una población $(\mathbf{P})$, una intervención que hay que evaluar (I), un comparador (C) y unos desenlaces (resultados, outcomes [0]) de interés (como por ejemplo la progresión de la enfermedad, la mortalidad o eventos adversos). El grupo elaborador define los desenlaces de interés que se van a evaluar y los clasifica en no importantes, importantes pero no clave para la toma de decisiones o desenlaces clave para la toma de decisiones. GRADE propone una escala de nueve puntos para realizar esta clasificación: de 1 a 3, desenlaces no importantes; de 4 a 6, desenlaces importantes pero no clave, y de 7 a 9 desenlaces clave para la toma de decisiones. Los desenlaces importantes y clave determinan la calidad global de la evidencia y el balance entre los beneficios y riesgos de las intervenciones evaluadas.

\subsection{Clasificación de la calidad de la evidencia}

La clasificación de la calidad de la evidencia se realiza para cada uno de los desenlaces de interés relevantes en la toma de decisiones $\mathrm{y}$, por tanto, para una pregunta clínica es posible que diferentes desenlaces presenten distintas calidades (1). La evaluación de la calidad de la evidencia y su clasificación nos permiten conocer el grado de confianza que tenemos en los resultados de la literatura.

GRADE propone clasificar la calidad de la evidencia como alta, moderada, baja o muy baja (1). GRADE considera que los ECA tiene una "calidad alta" y que los estudios observacionales tienen una "calidad baja". No obstante, GRADE sugiere una serie de aspectos que pueden reducir o aumentar esta calidad. Los aspectos que pueden reducir la calidad son las limitaciones de los estudios (en el diseño y ejecución [riesgo de sesgo]), los resultados inconsistentes o imprecisos, la ausencia de evidencia directa y el sesgo de publicación. Los aspectos que pueden aumentar la calidad son la fuerza de la asociación, el gradiente dosis-respuesta y si la consideración de otros 
factores de confusión potenciales que no se han tenido en cuenta en el estudio, si se presentasen, reforzarían sus resultados. La calidad de la evidencia para cada uno de los desenlaces de esta GPC, se presenta en el margen derecho del texto de síntesis de la evidencia.

A continuación se describen los aspectos mencionados (1):

- Limitaciones en el diseño o en la ejecución de los estudios (riesgo de sesgo): como la ausencia de ocultamiento de la secuencia de aleatorización, el enmascaramiento inadecuado, las pérdidas importantes, la ausencia de análisis por intención de tratar o la finalización del estudio antes de lo previsto por razones de beneficio, entre otros.

- Resultados inconsistentes: estimaciones discrepantes del efecto de un tratamiento (heterogeneidad o variabilidad en los resultados) en los estudios disponibles sugieren diferencias reales en dichas estimaciones. Estas pueden deberse a diferencias en la población, en la intervención, en los desenlaces o en la calidad de los estudios. Cuando existe heterogeneidad sin una potencial explicación razonable, la calidad disminuye.

- Ausencia de evidencia científica directa: en el caso de ausencia de comparaciones directas entre dos tratamientos (comparación de cada tratamiento frente a placebo, pero no entre ellos) o de encontrarse estudios realizados en poblaciones diferentes a la población objeto de la guía, se reduce la calidad. En el caso de la presente GPC, en donde se suelen localizar muy pocos estudios que evaluaron la progresión de la enfermedad (desenlace clave), la presión intraocular (PIO) se ha considerado evidencia indirecta de la progresión. Por tanto, se redujo la calidad de la evidencia.

- Imprecisión: cuando los estudios disponibles incluyen pocos eventos y pocos pacientes y presentan intervalos de confianza amplios, la calidad se reduce.

- Sesgo de notificación: la calidad puede disminuir si se tiene una duda razonable sobre si los autores han incluido todos los estudios (por ejemplo, el sesgo de publicación en el contexto de una RS) o si los autores han incluido todas las variables relevantes de resultado (outcome reporting bias).

Por otro lado, en situaciones poco frecuentes, cuando se han evaluado estudios observacionales y existe un bajo riesgo de sesgo, existen diversos factores que pueden aumentar su calidad: 
- Efecto importante: cuando el efecto observado muestra una asociación fuerte $(R R>2 \circ<0,5)$ o muy fuerte $(R R>5 \circ<0,2)$ y consistente, basada en estudios sin factores de confusión. En estas ocasiones, la calidad se puede considerar moderada o alta.

- La presencia de un gradiente dosis-respuesta.

- Situaciones en las que todos los posibles factores de confusión podrían haber reducido la asociación observada. En los casos en los que los pacientes que reciben la intervención de interés presentan un peor pronóstico y, aun así, mejores resultados que el grupo control, aumentan nuestra confianza en que el efecto potencial sea real.

Tras la clasificación de la calidad de la evidencia para cada uno de los desenlaces de interés, GRADE propone una evaluación de la calidad global. Para ello se sugiere tomar la calidad más baja de los desenlaces considerados críticos e importantes para la toma de decisiones (1). La calidad global para cada una de las preguntas se presenta en el apartado "De la evidencia a la recomendación".

\subsection{Graduación de la fuerza de las recomendaciones}

La fuerza de las recomendaciones nos informa de en qué medida el hecho de llevar a cabo una recomendación conlleva más beneficios que daños en los pacientes. GRADE propone varios factores que hay que tener en cuenta en la graduación de las recomendaciones $(2,4)$. Los principales son:

- Calidad de la evidencia: Si la calidad de la evidencia no es alta aunque la magnitud sea importante, debería reducirse la confianza y, por tanto, la fuerza con la que se lleve a cabo una recomendación.

- Valores y preferencias de los pacientes: Para incorporar la visión de los pacientes en la presente GPC, se integró a un representante de la AGAF en el grupo elaborador. Asimismo, se realizó una búsqueda de la literatura de estudios sobre valores y preferencias de los pacientes con glaucoma. Para cada una de las preguntas incluidas en la presente GPC, los principales hallazgos fueron descritos narrativamente en el apartado del texto "Valores y preferencias de los pacientes" y considerados en el apartado correspondiente de la sección "De la evidencia a la recomendación" de la pregunta.

- Balance entre beneficios y riesgos: Para realizar el balance entre los beneficios y los riesgos de las intervenciones evaluadas, deben considerarse las diferencias que existen entre los estimadores del efecto de los desenlaces considerados 
como claves e importantes. Si al realizar este balance existe una gran diferencia entre los beneficios (por ejemplo, disminución de la progresión de la enfermedad) y los riesgos (por ejemplo, efectos adversos), es más probable realizar una recomendación fuerte (a favor o en contra). Si la diferencia es pequeña, será más probable hacer una recomendación débil.

- Costes: A diferencia de otras variables de resultados, los costes son más variables en el tiempo, en distintas áreas geográficas y en función de diversas implicaciones. Así, aunque un coste elevado reduce la probabilidad de realizar una recomendación fuerte, el contexto en el que se realiza la recomendación será crítico en la valoración final de los mismos. En la presente GPC los autores han evaluado las preguntas y han decidido cuáles eran especialmente sensible a los costes. De un total de 31 comparaciones incluidas en la GPC, mediante la encuesta a los autores se evaluó la priorización de 24 de ellas. De las seis comparaciones no evaluadas, cinco se consideraron no sensibles a los costes (por ejemplo, factores de riesgo o fármacos de la misma familia) y una por considerarla sensible a costes (cribado). Finalmente, se realizó una búsqueda de la literatura de estudios de costes para 13 comparaciones. La síntesis de los resultados de los estudios económicos encontrados se incluyó en el apartado "Uso de recursos y costes" del texto de cada una de las preguntas y se consideró en el apartado de la sección "De la evidencia a la recomendación".

En la graduación de la fuerza de las recomendaciones, GRADE considera dos categorías: recomendaciones fuertes y débiles. En las recomendaciones fuertes, el grupo elaborador confía en que los efectos beneficiosos superan a los perjudiciales o viceversa, que los daños superan a los beneficios. En el primer caso, la recomendación es fuerte a favor. En el segundo, es fuerte en contra. Estas recomendaciones incluyen expresiones enfáticas del tipo "Se recomienda".

Las recomendaciones débiles también pueden ser a favor o en contra. Una recomendación es débil a favor cuando el grupo elaborador concluye que los efectos beneficiosos de llevar a cabo la recomendación superan los perjudiciales, aunque no está completamente seguro. En cambio, la recomendación es débil en contra cuando los efectos adversos probablemente superan a los beneficiosos. La recomendación se redacta con la expresión "Se puede valorar" o "Se sugiere".

En el apartado de la evidencia a la recomendación de cada una de las preguntas incluidas en esta GPC, se puede encontrar cómo el grupo elaborador ha tenido en cuenta estos aspectos en la elaboración de las recomendaciones. Para las 
intervenciones de las que no se dispone de evidencia, el grupo elaborador ha realizado recomendaciones de investigación con el fin de resaltar la importancia de realizar proyectos en áreas específicas.

Puede encontrarse información más detallada sobre la metodología GRADE en la web http: //www.gradeworkinggroup.org. Existen también otras publicaciones en castellano para usuarios de GPC: Alonso-Coello 2013a (5) y Alonso-Coello 2013b (6). 


\section{Preguntas en formato PICO}

\subsection{Factores de riesgo, despistaje y pruebas mínimas para el diagnóstico del glaucoma de ángulo abierto}

\begin{tabular}{|l|l|}
\hline \multicolumn{1}{|c|}{1.} & $\begin{array}{l}\text { En pacientes adultos, ¿cuáles son los factores de riesgo para } \\
\text { desarrollar glaucoma de ángulo abierto? }\end{array}$ \\
\hline \multirow{2}{*}{$\begin{array}{l}\text { Factor de } \\
\text { riesgo }\end{array}$} & Edad \\
\cline { 2 - 2 } & Sexo \\
\hline & Raza \\
\hline & PIO \\
\hline & Miopía \\
\hline & Diabetes \\
\hline & Migraña \\
\hline & Antecedentes familiares de glaucoma \\
\hline & Hábito tabáquico \\
\hline & Antecedentes de bypass de arteria coronaria o cirugía cardiovascular \\
\hline \multirow{2}{*}{ Comparación } & Población adulta \\
\hline Desenlaces & Desarrollo de glaucoma \\
\cline { 2 - 2 } & Progresión de la disminución del campo visual \\
\hline & Daño en el nervio óptico \\
\hline & Presión intraocular \\
\hline
\end{tabular}

2.

Espectro de pacientes

Prueba
En personas con factores de riesgo de glaucoma, ¿qué prueba o pruebas de cribado y con qué frecuencia se les deben realizar para diagnosticar precozmente el glaucoma de ángulo abierto?

Pacientes adultos con sospecha de GAA

-Oftalmoscopia

-Fotografía del disco óptico

-Valoración de la capa de fibras nerviosas mediante fotografía

-Tomografía retinal de Heidelberg II

-Perimetrías (perimetría de duplicación de frecuencia c-201-1 y c-20-5, perimetríaoculocinética, perimetría automatizada convencional supra umbral y umbral)

-Tonómetro de aplanación Goldmann

-Consenso de expertos:

Tonometría de no contacto (TNC) + SAP (múltiple stimuli) + HRT realizadas por un técnico/optometrista en la consulta de optometría Tonómetro ICare ${ }^{\circledR}$ + FDT + fotografía del disco óptico: realizadas por un técnico en la consulta de optometría

Tonometría (ICare ${ }^{\circledR}$ o Tono-pen o TNC) + FDT realizadas por un técnico en centros de cribado visual

TAG + SAP + fotografía del disco óptico realizadas por un técnico en 


\begin{tabular}{|c|c|}
\hline & $\begin{array}{l}\text { centros de cribado visual } \\
\text { Tonometría + imágenes reproducibles rápidamente o prueba de } \\
\text { función visual: realizadas por un técnico en centros de cribado visual y } \\
\text { en población con resultados de riesgo (risk scores) } \\
\text { TNC+ FDT + HRT o polarimetría con láser de barrido (GDx) realizadas } \\
\text { por un técnico en centros de cribado visual } \\
\text {-Otras técnicas: } \\
\text { Tomografía de coherencia óptica (OCT) } \\
\text { Polarimetría láser de barrido (GDx) } \\
\text { Polarimetría láser de barrido con compensación corneal variable (GDx- } \\
\text { VCC) } \\
\text { Analizador de grosor retiniano (RTA) } \\
\text { Campimetría automática de longitud de onda corta (SWAP) } \\
\text { Perimetría pulsar (PULSAR) } \\
\text { Umbrales de detección de movimiento (MDP) } \\
\text { Tonopen }\end{array}$ \\
\hline $\begin{array}{l}\text { Patrón de } \\
\text { referencia }\end{array}$ & Seguimiento clínico \\
\hline Desenlaces & $\begin{array}{l}\text { Variables de rendimiento diagnóstico: sensibilidad, especificidad, valor } \\
\text { predictivo positivo, valor predictivo negativo, DOR }\end{array}$ \\
\hline
\end{tabular}

3.

En personas con sospecha de glaucoma, ¿qué pruebas mínimas deberían realizarse para diagnosticar y clasificar su tipo de glaucoma?

\subsection{Tratamiento del glaucoma de ángulo abierto}

\subsubsection{Tratamiento farmacológico}

4. En los pacientes adultos, ¿qué tratamiento farmacológico se recomienda para el tratamiento del glaucoma de ángulo abierto?

\begin{tabular}{|c|c|}
\hline 5. & $\begin{array}{l}\text { En los pacientes adultos, ¿cuál de los siguientes fármacos: } \\
\text { parasimpaticomiméticos frente a betabloqueantes se recomienda } \\
\text { para el tratamiento del glaucoma de ángulo abierto? }\end{array}$ \\
\hline \multirow{2}{*}{$\begin{array}{l}\text { Población } \\
\text { Intervención/ } \\
\text { comparación }\end{array}$} & Paciente adulto con GAA \\
\hline & Parasimpáticomiméticos/betabloqueadores \\
\hline \multirow[t]{3}{*}{ Desenlaces } & Progresión del deterioro del campo visual \\
\hline & Presión intraocular \\
\hline & Efectos indeseables \\
\hline
\end{tabular}


6.

\section{Población}

Intervención/comparación

Desenlaces
En los pacientes adultos, ¿cuál de los siguientes fármacos: inhibidores de la anhidrasa carbónica frente a betabloqueantes se recomienda, para el tratamiento del glaucoma de ángulo abierto?

Paciente adulto con GAA

Inhibidores de la anhidrasa carbónica/betabloqueadores

Progresión del deterioro del campo visual

Presión intraocular

Efectos indeseables

\begin{tabular}{|c|c|}
\hline 7. & $\begin{array}{l}\text { En los pacientes adultos, ¿cuál de los siguientes } \\
\text { fármacos: análogos de las prostaglandinas frente a } \\
\text { betabloqueantes, se recomienda para el tratamiento } \\
\text { del glaucoma de ángulo abierto? }\end{array}$ \\
\hline Población & Paciente adulto con GAA \\
\hline Intervención/comparación & Análogos de las prostaglandinas/betabloqueadores \\
\hline \multirow[t]{3}{*}{ Desenlaces } & Progresión del deterioro del campo visual \\
\hline & Presión intraocular \\
\hline & Efectos indeseables \\
\hline
\end{tabular}

8.

Población

Intervención/

comparación

Desenlaces
En los pacientes adultos, ¿cuál de los siguientes fármacos: análogos de prostaglandinas frente a inhibidores de la anhidrasa carbónica, se recomienda para el tratamiento del glaucoma de ángulo abierto?

Paciente adulto con GAA

Análogos de las prostaglandinas/inhibidores de la anhidrasa carbónica

Progresión del deterioro del campo visual

Presión intraocular

Efectos indeseables
9.

Población

Intervención/comparación

Desenlaces
En los pacientes adultos, ¿cuál de los siguientes fármacos: betabloqueantes frente a agonista alfa2 selectivos, se recomienda para el tratamiento del glaucoma de ángulo abierto?

Paciente adulto con GAA

Betabloqueadores/agonistas alfa selectivos

Progresión del deterioro del campo visual

Presión intraocular

Efectos indeseables 
10.

Población

Intervención/comparación

Desenlaces
En los pacientes adultos, ¿cuál de los siguientes fármacos análogos de las prostaglandinas se recomienda para el tratamiento del glaucoma de ángulo abierto?

\section{Paciente adulto con GAA}

Análogos de las prostaglandinas/análogos de las prostaglandinas

Progresión del deterioro del campo visual

Presión intraocular

Efectos indeseables
11.

Población

Intervención/comparación

Desenlaces
En los pacientes adultos, ¿cuál de los siguientes fármacos: análogos de prostaglandinas frente a agonistas alfa2 selectivos se recomienda para el tratamiento del glaucoma de ángulo abierto?

Paciente adulto con GAA

Análogos de las prostaglandinas/agonistas alfa selectivos

Progresión del deterioro del campo visual

Presión intraocular

Efectos indeseables
12.

\section{Población}

Intervención/comparación

Desenlaces
En los pacientes adultos, ¿cuál de los siguientes fármacos betabloqueantes se recomienda para el tratamiento del glaucoma de ángulo abierto?

Paciente adulto con GAA

Betabloqueadores/betabloqueadores

Progresión del deterioro del campo visual

Presión intraocular

Efectos indeseables
13.

\section{Población \\ Intervención \\ Comparación \\ Desenlaces}

En los pacientes adultos, ¿se recomienda el uso de combinaciones fijas de fármacos frente el uso de combinaciones no fijas de estos fármacos, para el tratamiento del glaucoma de ángulo abierto?

Paciente adulto con GAA

Combinaciones fijas de dos fármacos

Fármacos tópicos en administración individual

Progresión del deterioro del campo visual

Presión intraocular

Adherencia al tratamiento

Efectos indeseables 
14.

En los pacientes adultos, ¿se recomienda el uso de fármacos libres de conservantes frente el uso de fármacos con conservantes, para el tratamiento del glaucoma de ángulo abierto?

Población Paciente adulto con GAA

Intervención

Preparados tópicos con uno o más fármacos sin conservantes

Comparación

Preparados tópicos con uno o más fármacos (combinación fija) con conservantes

Desenlaces Progresión del deterioro del campo visual

Presión intraocular

Efectos indeseables

\subsubsection{Tratamiento con láser}

15.

En los pacientes adultos, ¿se recomienda la trabeculoplastia con láser para el glaucoma de ángulo abierto?

16.

En pacientes adultos, ¿se recomienda la trabeculoplastia con láser diodo respecto a la trabeculoplastia con láser argón para el tratamiento del glaucoma de ángulo abierto?

Población Paciente adulto con GAA

Intervención

Trabeculoplastia con láser diodo

Comparación Trabeculoplastia con láser argón

Desenlaces Progresión del deterioro del campo visual

Presión intraocular

Efectos indeseables

17.

En pacientes adultos, ¿se recomienda la trabeculoplastia con láser selectivo respecto a la trabeculoplastia con láser argón para el tratamiento del glaucoma de ángulo abierto?

Población Paciente adulto con GAA

Intervención/ Trabeculoplastia con láser selectivo

Comparación

Desenlaces

Trabeculoplastia con láser argón

Progresión del deterioro del campo visual

Presión intraocular

Efectos indeseables 
18.

En pacientes adultos, ¿se recomienda la trabeculoplastia con láser respecto al tratamiento farmacológico para el tratamiento del glaucoma de ángulo abierto?

Población Paciente adulto con GAA

Intervención

Comparación

Trabeculoplastia con láser (argón, selectivo y diodo)

Tratamiento farmacológico

Desenlace

Progresión del deterioro del campo visual

Presión intraocular

Efectos indeseables

19.

En los pacientes adultos, ¿se recomienda la trabeculoplastia con láser asociada a tratamiento farmacológico respecto al tratamiento farmacológico para el tratamiento del glaucoma de ángulo abierto?

Población Paciente adulto con GAA

Intervención

Trabeculoplastia con láser más tratamiento farmacológico

Comparación

Tratamiento farmacológico

Desenlaces

Progresión del deterioro del campo visual

Presión intraocular

Efectos indeseables

20.

En los pacientes adultos, ¿se recomienda la trabeculoplastia con láser respecto a la trabeculectomía para el tratamiento del glaucoma de ángulo abierto?

Población $\quad$ Paciente adulto con GAA

Intervención Trabeculoplastia con láser (argón, selectivo y diodo)

Comparación Trabeculectomía

Desenlaces Progresión del deterioro del campo visual

Presión intraocular

Efectos indeseables

\subsubsection{Tratamiento quirúrgico}

21.

En los pacientes adultos, ¿qué tratamiento quirúrgico se recomienda para el tratamiento glaucoma de ángulo abierto? 
22.

En pacientes adultos, ¿se recomienda la trabeculectomía respecto a tratamiento farmacológico para el tratamiento del glaucoma de ángulo abierto?

Población Paciente adulto con GAA

Intervención Trabeculectomía

Comparación

Desenlaces

Tratamiento farmacológico

Progresión del deterioro del campo visual

Presión intraocular

Efectos indeseables

23.

En pacientes adultos, ¿se recomienda la trabeculectomía asociada a antimetabolito respecto a la trabeculectomía sola, para el tratamiento del glaucoma de ángulo abierto?

Población Paciente adulto con GAA

Intervención

Trabeculectomía más antimetabolitos

Comparación

Desenlaces

Trabeculectomía

Progresión del deterioro del campo visual

Presión intraocular

Efectos indeseables

24.

En pacientes adultos, ¿se recomienda la cirugía no penetrante respecto a la trabeculectomía, para el tratamiento del glaucoma de ángulo abierto?

Población Paciente adulto con GAA

Intervención

Cirugía no penetrante (esclerectomía profunda y viscocanalostomía)

Comparación

Trabeculectomía

Desenlaces

Progresión del deterioro del campo visual

Presión intraocular

Efectos indeseables

25.

En pacientes adultos, ¿se recomienda la cirugía no penetrante asociada a antimetabolitos respecto a la cirugía no penetrante sola, para el tratamiento del glaucoma de ángulo abierto?

Población Paciente adulto con GAA

Intervención

Cirugía no penetrante más antimetabolitos

Comparación

Desenlaces

Cirugía no penetrante aislada

Progresión del deterioro del campo visual

Presión intraocular

Efectos indeseables 


\begin{tabular}{|l|l|}
\hline \multicolumn{1}{|c|}{26.} & $\begin{array}{l}\text { En los pacientes adultos, ¿se recomienda el uso de } \\
\text { viscocanalostomía frente a la esclerectomía profunda, para el } \\
\text { tratamiento del glaucoma de ángulo abierto? }\end{array}$ \\
\hline Población & Paciente adulto con GAA \\
\hline Intervención & Viscocanalostomía \\
\hline Comparación & Esclerectomía profunda \\
\hline Desenlaces & Progresión del deterioro del campo visual \\
\hline & Presión intraocular \\
\hline & Efectos indeseables \\
\hline
\end{tabular}

\begin{tabular}{|l|l|}
\hline \multicolumn{1}{|c|}{27.} & $\begin{array}{l}\text { En pacientes adultos, ¿se recomienda la cirugía no penetrante } \\
\text { sin implantes respecto a la cirugía no penetrante con implantes, } \\
\text { para el tratamiento del glaucoma de ángulo abierto? }\end{array}$ \\
\hline Población & Paciente adulto con GAA \\
\hline Intervención & Cirugía no penetrante sin implantes \\
\hline Comparación & Cirugía no penetrante con implantes \\
\hline Desenlaces & Progresión del deterioro del campo visual \\
\hline & Presión intraocular \\
\hline & Efectos indeseables \\
\hline
\end{tabular}

28.

En pacientes adultos, ¿se recomienda el uso de implante ExPress respecto a la trabeculectomía, para el tratamiento del glaucoma de ángulo abierto?

\begin{tabular}{l|l} 
Población & Paciente adulto con GAA \\
\hline Intervención & Implante Ex-Press \\
\hline Comparación & Trabeculectomía \\
\hline Desenlaces & Progresión del deterioro del campo visual \\
\cline { 2 - 2 } & Presión intraocular \\
\cline { 2 - 2 } & Efectos indeseables
\end{tabular}

29.

En pacientes adultos, ¿se recomienda a los dispositivos de drenaje (valvulados o no) respecto a la trabeculectomía, para el tratamiento del glaucoma de ángulo abierto?

Población Paciente adulto con GAA

Intervención Dispositivos de drenaje (valvulados o no)

Comparación Trabeculectomía

Desenlaces Progresión del deterioro del campo visual Presión intraocular Efectos indeseables 
30.

En pacientes adultos, ¿se recomienda microtrabeculectomia respecto a la trabeculectomía para, el tratamiento del glaucoma de ángulo abierto?

Población Paciente adulto con GAA Intervención Microtrabeculectomía Comparación Desenlaces

Trabeculectomía

Progresión del deterioro del campo visual

Presión intraocular

Efectos indeseables

31.

En pacientes adultos, ¿se recomienda las técnicas microinvasivas (MICS) respecto a la trabeculectomía, para el tratamiento del glaucoma de ángulo abierto?

Población Paciente adulto con GAA

Intervención Técnicas microinvasivas (MICS)

Comparación Trabeculectomía

Desenlaces

Progresión del deterioro del campo visual

Presión intraocular

Efectos indeseables

\subsection{Anestesia en cirugía penetrante y no penetrante de glaucoma}

32.

\section{Población} Intervención Comparación Desenlaces
En los pacientes adultos, ¿qué técnica de anestesia locoregional se recomienda en la cirugía del glaucoma de ángulo abierto?

Pacientes adultos con GAA

Anestesia tópica

Anestesia retrobulbar

Dolor

Uso de anestesia adicional durante la cirugía

Efectos indeseables
33.

En los pacientes adultos, ¿se recomienda la anestesia tópica respecto a la anestesia retrobulbar en la cirugía de glaucoma de ángulo abierto?

Población Intervención

Comparación

Desenlaces

\section{Pacientes adultos con GAA}

Anestesia tópica

Anestesia subtenoniana

Dolor

Uso de anestesia adicional durante la cirugía

Tasa de éxito (completo y calificado a los 4 años)

Efectos indeseables 
34.

En los pacientes adultos, ¿se recomienda la anestesia tópica respecto a la anestesia subtenoniana en la cirugía de glaucoma de ángulo abierto?

Población Intervención Comparación Desenlaces Pacientes adultos con GAA Anestesia tópica

Anestesia peribulbar

Dolor

Uso de anestesia adicional durante la cirugía

Efectos indeseables

35.

En los pacientes adultos, ¿se recomienda la anestesia tópica respecto a la anestesia peribulbar en la cirugía de glaucoma de ángulo abierto?

Población Pacientes adultos con GAA

Intervención

Anestesia de contacto

Comparación

Desenlaces

Otras técnicas anestésicas (peribulbar/tópica)

Dolor

Presión intraocular

Uso de anestesia adicional durante la cirugía

Efectos indeseables

36.

En los pacientes adultos, ¿se recomienda la anestesia de contacto respecto a otras técnicas anestésicas en la cirugía de glaucoma de ángulo abierto?

Población

Pacientes adultos con GAA

Intervención Anestesia subtenoniana

Comparación Anestesia retrobulbar

Desenlaces Molestias oculares

Uso de analgesia postoperatoria

Efectos indeseables

37.

En los pacientes adultos, ¿se recomienda la anestesia subtenoniana respecto a la anestesia retrobulbar en la cirugía de glaucoma de ángulo abierto?

2.4. Control evolutivo del paciente adulto con GAA

En el control evolutivo de los adultos con glaucoma de ángulo abierto, ¿qué exámenes de seguimiento se realizan y con qué

38. frecuencia deben hacerse? 
Duración de las intervenciones:

1. Tratamiento médico: 2-3 meses.

2. Tratamiento láser: 2-3 meses.

3. Cirugía: 1 año.

Para efectos secundarios: 6 meses.

\section{Estrategias de búsqueda}

\section{Estrategias de búsqueda}

Para el desarrollo de las estrategias de búsqueda, se toma como referencia la GPC de la NICE sobre el diagnóstico y manejo del glaucoma crónico de ángulo abierto y de la hipertensión ocular publicada (2009) (47). La fecha de última búsqueda de la GPC de la NICE fue el 4 de agosto de 2008. Para las estrategias de búsqueda de la literatura de la presente GPC, se utilizan filtros para obtener revisiones sistemáticas y ensayos clínicos aleatorizados y se pone como límite temporal el 1 de enero de 2008.

Para las preguntas no incluidas en la GPC de la NICE, se desarrollan estrategias específicas para responderlas.

1. Cribado, diagnóstico, farmacológico, láser y cirugía del glaucoma de ángulo abierto

- Base de datos: MEDLINE (PubMed).

- Fecha búsqueda: 12.09.2013.

\#1 glaucoma[tiab]

\#2 systematic[sb]

\#3 \#1 AND \#2

\#5 trial [ti]

\#6 randomly[tiab]

\#7 clinical trials as topic[mesh: noexp]

\#8 placebo[tiab]

\#9 randomized[tiab]

\#10 controlledclinical trial[pt]

\#11 randomizedcontrolled trial[pt]

\#12 \#5 OR \#6 OR \#7 OR \#8 OR \#9 OR \#10 OR \#11

\#13 animals [mh] NOT humans [mh] 
\#14 \#12 NOT \#13

\#15 \#1 AND \#14

- Base de datos: TheCochraneLibrary (incluye NHS EED, DARE, CENTRAL).

- Fecha de búsqueda: 26.09.2013.

\#1 MeSH descriptor: [Glaucoma] explode all trees 2098

\#2 glaucoma: ti, ab

\#3 \#1 or \#2

2. Combinaciones fijas de fármacos frente a combinaciones no fijas de fármacos

Pregunta no incluida en la GPC NICE (47), por lo que se desarrollo una estrategia de búsqueda específica para responderla.

- Base de datos: MEDLINE (PubMed).

- Fecha de búsqueda: 27.09.2013.

\#1 “Glaucoma”[MeSH]

\#2 glaucoma[tiab]

\#3 \#1 OR \#2

\#4 intraocularpressure[tiab]

\#5 ocular hypotens*[tiab]

\#6 ocular hypertens*[tiab]

\#7 antiglaucoma[tiab]

\#8 "Antihypertensive Agents/administrationanddosage"[Mesh]

\#9 “Antihypertensive Agents/therapeuticuse"[MeSH]

\#10 “Ocular Hypertension/drugtherapy"[MeSH]

\#11 \#4 OR \#5 OR \#6 OR \#7 OR \#8 OR \#9 OR \#10

\#12 unfixed[tiab]

\#13 non fixed[tiab]

\#14 concomitant $^{\star}[$ tiab] 
\#15 \#12 OR \#13 OR \#14

\#16 fixed ${ }^{*}[$ tiab]

\#17 \#3 AND \#11 AND \#15 AND \#16

- Base de datos: TheCochraneLibrary (incluye NHS EED, DARE, CENTRAL).

- Fecha de búsqueda: 27.09.2013.

\#1 MeSH descriptor: [Glaucoma] explode all trees

\#2 glaucoma: ti, ab, kw

\#3 \#1 or \#2

\#4 intraocularpressure: ti, ab

\#5 ocular hypotens*: ti, ab

\#6 ocular hypertens*: ti, ab

\#7 antiglaucoma: ti, ab

\#8 MeSH descriptor: [Antihypertensive Agents] explode all treesandwithqualifiers: [Administration\&dosage-AD, Therapeuticuse-TU]

\#9 MeSH descriptor: [Ocular Hypertension] explode all treesandwithqualifiers: [Drugtherapy-DT]

\#10 $\quad$ \{or \#4-\#9\}

\#11 unfixed: ti, ab

\#12 non fixed: ti, ab

\#13 concomitant*: ti, ab

\#14 $\quad$ or \#11-\#13\}

\#15 fixed*: ti, ab

\#16 \#3 and \#10 and \#14 and \#15

\section{Fármacos libres de preservantes frente a fármacos con preservantes}

Pregunta no incluida en la GPC NICE (47), por lo que se desarrolló una estrategia de búsqueda específica para responderla.

- Base de datos: MEDLINE (PubMed). 
- Fecha de búsqueda: 27.09.2013.

\#1 "Glaucoma”[MeSH]

\#2 glaucoma[tiab]

\#3 ocular hypertens*[tiab]

\#4 intraocularpressure[tiab]

\#5 \#1 OR \#2 OR \#3 OR \#4

\#6 "Antihypertensive Agents/administrationanddosage"[Mesh]

\#7 “Antihypertensive Agents/therapeuticuse"[MeSH]

\#8 formulation*[tiab]

\#9 combination*[tiab]

\#10 $\quad$ treat*[tiab]

\#11 \#6 or \#7 or \#8 or \#9 or \#10

\#12 preservative*[tiab]

\#13 "Preservatives, Pharmaceutical”[MeSH]

\#14 \#12 or \#13

\#15 \#5 and \#11 and \#14

- Base de datos: TheCochraneLibrary (incluye NHS EED, DARE, CENTRAL).

- Fecha de búsqueda: 27.09.2013.

\#1 MeSH descriptor: [Glaucoma] explode all trees

\#2 glaucoma: ti, ab, kw

\#3 intraocularpressure: ti, ab

\#4 ocular hypertens*: ti, ab

\#5 MeSH descriptor: [Antihypertensive Agents] explode all treesandwithqualifiers:

[Administration\&dosage-AD, Therapeuticuse-TU]

\#6 formulation*: ti, ab

\#7 combination*: ti, ab

\#8 treat*: ti, ab

\#9 \{or \#1-\#4\} 


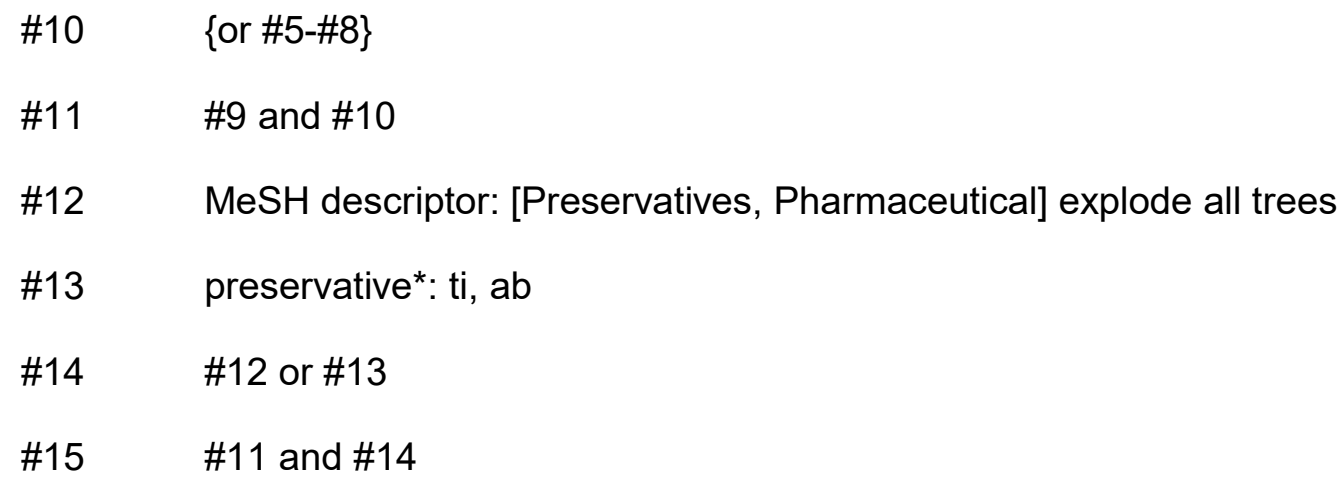

\section{Anestesia en la cirugía del glaucoma de ángulo abierto}

Pregunta no incluida en la GPC NICE (47), por lo que se desarrolló una estrategia de búsqueda específica para responderla.

- Base de datos: MEDLINE (PubMed).

- Fecha de búsqueda: 18.12.2013.

\#1 $\left(\left(\left(\right.\right.\right.$ anaesthe ${ }^{*}[$ tiab] OR anesthe*[tiab])) AND $(((()(((()($ Contact $[$ tiab]) OR Topical[tiab]) OR retrobulbar[tiab]) OR peribulbar[tiab]) OR intracameral[tiab]) OR subtenon) OR sub-tenon*[tiab]) OR ocular[tiab]) OR intraconal[tiab]) OR extraconal[tiab]) OR periocular[tiab]) OR General[tiab])) AND ((glaucoma[tiab]) OR Trabeculectomy[tiab])

\#2 systematic[sb] OR "Meta-Analysis"[pt]

\#3 (randomizedcontrolled trial[pt] OR controlledclinical trial[pt] OR randomized[tiab] OR placebo[tiab] OR drugtherapy[sh] OR randomly[tiab] OR trial[tiab] OR groups[tiab]) NOT (animals [mh] NOT humans [mh])

\#4 \#1 AND \#2

\#5 \#1 AND \#3

\#6 \#5 NOT \#4

- Base de datos: TheCochraneLibrary (incluye NHS EED, DARE, CENTRAL).

- Fecha de búsqueda: 18.12.2013.

\#1 MeSH descriptor: [Glaucoma] explode all trees

\#2 glaucoma: ti, ab, kw

\#3 intraocularpressure: ti, ab

\#4 ocular hypertens*: ti, ab

\#5 MeSH descriptor: [Trabeculectomy] explode all trees 
\#6 trabeculectomy: ti, ab

$\# 7$ \#1 or \#2 or \#3 or \#4 or \#5 or \#6

\#8 MeSH descriptor: [Anesthesia] explode all trees

\#9 anaesthe* ${ }^{*}$ ti, ab

\#10 anesthe*: $\mathrm{ti}, \mathrm{ab}$

\#11 \#8 or \#9 or \#10

\#12 contact: ti, ab

\#13 topic*: ti, ab

\#14 retrobulbar: ti, ab

\#15 peribulbar: ti, ab

\#16 intracameral: ti, ab

\#17 (subneartenon*): ti, ab

\#18 ocular: ti, ab

\#19 intraconal: ti, ab

\#20 extraconal: ti, ab

\#21 periocular: ti, ab

\#22 intraconal: ti, ab

\#23 extra conal: ti, ab

\#24 periocular: ti, ab

\#25 general: ti, ab 28810

$\# 26 \quad \# 12$ or \#13 or \#14 or \#15 or \#16 or \#17 or \#18 or \#19 or \#20 or \#21 or $\# 22$ or \#23 or \#24 or \#25

\#27 \#7 and \#11

\#28 \#27 and \#26

\#29 \#27 not \#28 


\section{Resultados}

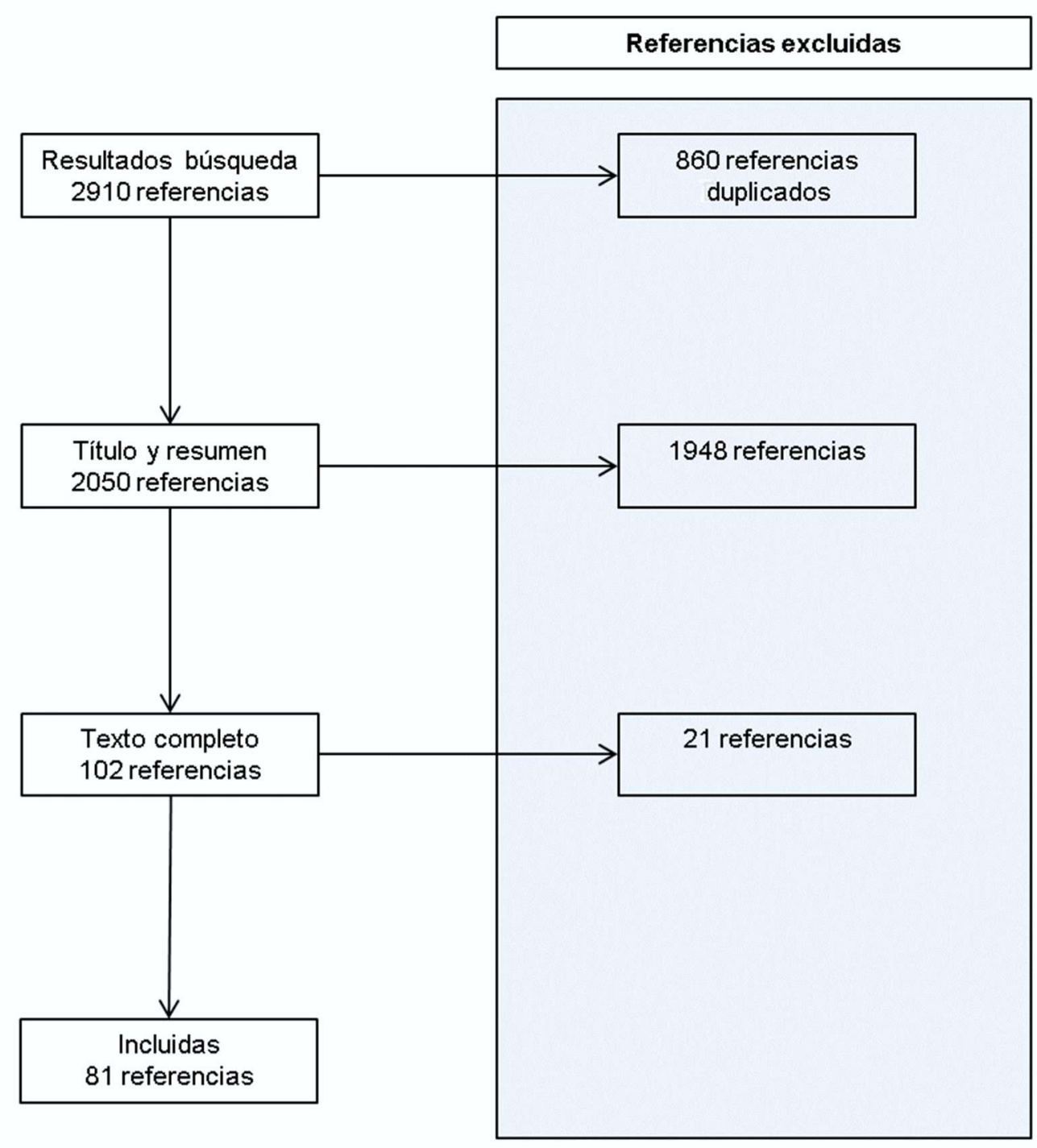




\section{Tablas GRADE}

\subsubsection{Factores de riesgo para el desarrollo del glaucoma de ángulo abierto}

Question: Should Demographic risk factors be used in open angle glaucoma?

Settings:

Bibliography:

Burr JM, Mowatt G, Hernández R, Siddiqui MA, Cook J, Lourenco T, et al. The clinical effectiveness and cost-effectiveness of screening for open angle glaucoma: a systematic review and economic evaluation. Health technology assessment (Winchester, Inglaterra). 2007; 11(41): iii-iv, ix-x, 1190.

Hollands H, Johnson D, Hollands S, Simel DL, Jinapriya D, Sharma S. Do findings on routine examination identify patients at risk for primary openangle glaucoma? The rational clinical examination systematic review. JAMA. 2013; 309(19): 2035-42.

\begin{tabular}{|c|c|c|c|c|c|c|c|c|c|c|c|c|}
\hline \multicolumn{7}{|c|}{ Quality assessment } & \multicolumn{2}{|c|}{ No of patients } & \multicolumn{2}{|c|}{ Effect } & \multirow{2}{*}{ Quality } & \multirow{2}{*}{ Importance } \\
\hline $\begin{array}{c}\text { No of } \\
\text { studies }\end{array}$ & Design & $\begin{array}{c}\text { Risk of } \\
\text { bias }\end{array}$ & Inconsistency & Indirectness & Imprecision & \begin{tabular}{|c|} 
Other \\
considerations
\end{tabular} & $\begin{array}{c}\text { Demographic } \\
\text { risk factors }\end{array}$ & Control & \begin{tabular}{|l|} 
Relative \\
$(95 \% \mathrm{Cl})$
\end{tabular} & Absolute & & \\
\hline \multicolumn{13}{|c|}{ Age $>80$ year (Holland 2013) } \\
\hline 13 & $\begin{array}{l}\text { observational } \\
\text { studies }\end{array}$ & serious $^{1}$ & $\begin{array}{l}\text { no serious } \\
\text { inconsistency }\end{array}$ & $\begin{array}{l}\text { no serious } \\
\text { indirectness }\end{array}$ & $\begin{array}{l}\text { no serious } \\
\text { imprecision }\end{array}$ & none $^{2}$ & - & $0 \%$ & $\begin{array}{c}\text { OR } 2.9 \\
(1.9 \text { to } \\
4.3)\end{array}$ & - & $\begin{array}{c}\square \square \square \square \\
\text { MODERATE }\end{array}$ & \\
\hline \multicolumn{13}{|c|}{ Male sex (Holland 2013) } \\
\hline 20 & $\begin{array}{l}\text { observational } \\
\text { studies }\end{array}$ & serious $^{1}$ & $\begin{array}{l}\text { no serious } \\
\text { inconsistency }\end{array}$ & $\begin{array}{l}\text { no serious } \\
\text { indirectness }\end{array}$ & $\begin{array}{l}\text { no serious } \\
\text { imprecision }\end{array}$ & none & - & $0 \%$ & $\begin{array}{c}\text { OR } 1.2 \\
(0.97 \text { to } \\
1.4)\end{array}$ & - & $\begin{array}{c}\text { MU⿴囗十 } \\
\text { MODERATE }\end{array}$ & \\
\hline
\end{tabular}




\section{Ethnicity (Burr 2007)}

observational no

studies

no serious

no serious

no serious none

serious

inconsistency indirectness

imprecision

bias

1 Studies included published between 1966 and 2011. Disease definition varied over that time. The included studies represented a large number of patients across many different populations with different glaucoma prevalence.

2 Increasing age is associated with a significantly higher risk of OAG. 


\section{Bibliography:}

Marcus MW, de Vries MM, Junoy Montolio FG, Jansonius NM. Myopia as a risk factor for open-angle glaucoma: a systematic review and metaanalysis. Ophthalmology. 2011; 118(10): 1989-1994.e2.

Hollands H, Johnson D, Hollands S, Simel DL, Jinapriya D, Sharma S. Do findings on routine examination identify patients at risk for primary openangle glaucoma? The rational clinical examination systematic review. JAMA. 2013; 309(19): 2035-42.

\begin{tabular}{|c|c|c|c|c|c|c|c|c|c|c|c|c|}
\hline \multicolumn{7}{|c|}{ Quality assessment } & \multicolumn{2}{|c|}{ No of patients } & \multicolumn{2}{|c|}{ Effect } & \multirow{2}{*}{ Quality } & \multirow{2}{*}{ Importance } \\
\hline $\begin{array}{l}\text { No of } \\
\text { studies }\end{array}$ & Design & $\begin{array}{l}\text { Risk of } \\
\text { bias }\end{array}$ & Inconsistency & Indirectness & Imprecision & $\begin{array}{c}\text { Other } \\
\text { considerations }\end{array}$ & $\begin{array}{l}\text { Ocular } \\
\text { Risk } \\
\text { Factor }\end{array}$ & Control & $\begin{array}{l}\text { Relative } \\
(95 \% \mathrm{Cl})\end{array}$ & Absolute & & \\
\hline \multicolumn{13}{|c|}{ Presión intraocular (Burr 2007) } \\
\hline 7 & $\begin{array}{l}\text { observational } \\
\text { studies }\end{array}$ & serious ${ }^{1}$ & $\begin{array}{l}1 \text { no serious } \\
\text { inconsistency }\end{array}$ & $\begin{array}{l}\text { no serious } \\
\text { indirectness }\end{array}$ & $\begin{array}{l}\text { no serious } \\
\text { imprecision }{ }^{2}\end{array}$ & none & - & $0 \%$ & $\begin{array}{c}\text { OR } 30.65 \\
(13.22 \text { to } \\
71.05)\end{array}$ & - & $\begin{array}{c}\square \square \square \square \\
\text { MODERATE }\end{array}$ & \\
\hline \multicolumn{13}{|c|}{ Myopia (Marcus 2007) } \\
\hline 9 & $\begin{array}{l}\text { observational } \\
\text { studies }\end{array}$ & serious $^{3}$ & 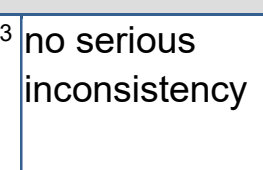 & $\begin{array}{l}\text { no serious } \\
\text { indirectness }\end{array}$ & \begin{tabular}{|l} 
no serious \\
imprecision
\end{tabular} & none & - & $0 \%$ & \begin{tabular}{|c|} 
OR 1.88 \\
$(1.6$ to \\
$2.2)$
\end{tabular} & - & 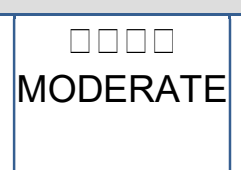 & \\
\hline \multicolumn{13}{|c|}{ Low myopia I (Marcus 2011) (assessed with: myopia with a spherical equivalent refractive error up to -3 diopters) } \\
\hline 6 & $\begin{array}{l}\text { observational } \\
\text { studies }\end{array}$ & serious $^{3}$ & $\begin{array}{l}3 \text { no serious } \\
\text { inconsistency }\end{array}$ & $\begin{array}{l}\text { no serious } \\
\text { indirectness }\end{array}$ & $\begin{array}{l}\text { no serious } \\
\text { imprecision }\end{array}$ & none & - & $0 \%$ & \begin{tabular}{|c} 
OR 1.77 \\
$(1.41$ to \\
$2.23)$
\end{tabular} & - & $\begin{array}{c}\square \square \square \square \\
\text { MODERATE }\end{array}$ & \\
\hline
\end{tabular}




\begin{tabular}{|c|c|c|c|c|c|c|c|c|c|c|c|}
\hline 7 & $\begin{array}{l}\text { observational } \\
\text { studies }\end{array}$ & serious $^{3}$ & $\begin{array}{l}\text { no serious } \\
\text { inconsistency }\end{array}$ & $\begin{array}{l}\text { no serious } \\
\text { indirectness }\end{array}$ & $\begin{array}{l}\text { no serious } \\
\text { imprecision }\end{array}$ & none & - & $0 \%$ & \begin{tabular}{|c|} 
OR 2.46 \\
$(1.93$ to \\
$3.15)$
\end{tabular} & - & $\begin{array}{c}\square \square \square \square \\
\text { MODERATE }\end{array}$ \\
\hline \multicolumn{12}{|c|}{ High myopia II (Holland 2013) (assessed with: myopia with a spherical equivalent refractive error greater or equal to -8 diopters) } \\
\hline 1 & $\begin{array}{l}\text { observational } \\
\text { studies }\end{array}$ & serious $^{4}$ & $\begin{array}{l}\text { no serious } \\
\text { inconsistency }\end{array}$ & $\begin{array}{l}\text { no serious } \\
\text { indirectness }\end{array}$ & serious $^{5}$ & none & - & $0 \%$ & $\begin{array}{c}\text { OR } 5.0 \\
(2.2 \text { to } 11)\end{array}$ & - & $\begin{array}{l}\square \square \square \square \\
\text { LOW }\end{array}$ \\
\hline
\end{tabular}

${ }^{1}$ Studies included published mora than 10 years of interval. Disease definition varied over that time. All studies but one included cases with previously detected OAG, and thus already under glaucoma therapy, and there fore underestimate the true prevalence of OAG in people with high IOP. Previous treatments with laser (refractive surgery) unclear and could alter the IOP results.

${ }^{2}$ Imprecision was not downgraded even with a large IC because the lower boundary of the confidence interval warrants a clinical intervention.

${ }^{3}$ The way patients were diagnosed with myopia and OAG varied between the studies. The OAG definition was based on a combination of

glaucomatous visual field loss and optic disc abnormalities in most studies, with various criteria and cutoff points. The cutoff point between low and high myopia caried between -3 and $-4 D$ (-3D in most studies), and the cutoff point between emmetropia and (low) myopia varied between $-0,001$ and $-1,5 \mathrm{D}(-0,5$ or $-1 \mathrm{D}$ in most studies).

${ }^{4}$ Studies included published between 1966 and 2011. Disease definition varied over that time. The included studies represented a large number of patients across many different populations with different glaucoma prevalence.

${ }^{5}$ Wide $95 \%$ confidence interval. 


\section{Bibliography:}

Topouzis F, Wilson MR, Harris A, Founti P, Yu F, Anastasopoulos E, et al. Risk factors for primary open-angle glaucoma and pseudoexfoliative glaucoma in the Thessaloniki eye study.Am J Ophthalmol. 2011; 152(2): 219-228.e1.

Burr JM, Mowatt G, Hernández R, Siddiqui MA, Cook J, Lourenco T, et al. The clinical effectiveness and cost-effectiveness of screening for open angle glaucoma: a systematic review and economic evaluation. Health technology assessment (Winchester, Inglaterra). 2007; 11(41): iii-iv, ix-x, 1190.

Edwards R, Thornton J, Ajit R, Harrison RA, Kelly SPJ. Cigarette smoking and primary open angle glaucoma: a systematic review. Glaucoma. 2008; 17(7): 558-66.

Hollands H, Johnson D, Hollands S, Simel DL, Jinapriya D, Sharma S. Do findings on routine examination identify patients at risk for primary openangle glaucoma? The rational clinical examination systematic review. JAMA. 2013; 309(19): 2035-42.

\begin{tabular}{|c|c|c|c|c|c|c|c|c|c|c|c|c|}
\hline \multicolumn{7}{|c|}{ Quality assessment } & \multicolumn{2}{|c|}{ No of patients } & \multicolumn{2}{|c|}{ Effect } & \multirow{2}{*}{ Quality } & \multirow{2}{*}{ Importance } \\
\hline $\begin{array}{l}\text { No of } \\
\text { studies }\end{array}$ & Design & $\begin{array}{l}\text { Risk of } \\
\text { bias }\end{array}$ & Inconsistency & Indirectness & Imprecision & $\begin{array}{c}\text { Other } \\
\text { considerations }\end{array}$ & $\begin{array}{c}\text { Non- } \\
\text { ocular } \\
\text { risk } \\
\text { factors }\end{array}$ & Control & $\begin{array}{l}\text { Relative } \\
(95 \% \mathrm{Cl})\end{array}$ & Absolute & & \\
\hline \multicolumn{13}{|c|}{ Diabetes (Burr 2007) } \\
\hline 3 & $\begin{array}{l}\text { observational } \\
\text { studies }\end{array}$ & serious $^{1}$ & $\begin{array}{l}\text { no serious } \\
\text { inconsistency }\end{array}$ & $\begin{array}{l}\text { no serious } \\
\text { indirectness }\end{array}$ & $\begin{array}{l}\text { no serious } \\
\text { imprecision }\end{array}$ & none & - & $0 \%$ & $\begin{array}{c}\text { RR } 2.08 \\
(1.44 \text { to } \\
2.99)\end{array}$ & 1 & \begin{tabular}{c|}
$\square \square \square$ \\
MODERATE
\end{tabular} & \\
\hline \multicolumn{13}{|c|}{ Diabetes (Holland 2013) } \\
\hline 6 & $\begin{array}{l}\text { observational } \\
\text { studies }\end{array}$ & serious ${ }^{4}$ & $\begin{array}{l}\text { no serious } \\
\text { inconsistency }\end{array}$ & \begin{tabular}{|l|} 
no serious \\
indirectness
\end{tabular} & $\begin{array}{l}\text { no serious } \\
\text { imprecision }\end{array}$ & none & & & $\begin{array}{l}\text { OR } 1.8 \\
\text { (1.4 to }\end{array}$ & - & $\begin{array}{c}\text { पि口 } \\
\text { VERY LOW }\end{array}$ & \\
\hline
\end{tabular}




\begin{tabular}{|c|c|c|c|c|c|c|c|c|c|c|c|c|}
\hline & & & & & & & & & 2.4) & & & \\
\hline \multicolumn{13}{|c|}{ Family history (Burr 2007) } \\
\hline 3 & $\begin{array}{l}\text { observational } \\
\text { studies }\end{array}$ & serious $^{1}$ & $\begin{array}{l}\text { no serious } \\
\text { inconsistency }\end{array}$ & $\begin{array}{l}\text { no serious } \\
\text { indirectness }\end{array}$ & $\begin{array}{l}\text { no serious } \\
\text { imprecision }\end{array}$ & none & - & $0 \%$ & $\begin{array}{c}\text { RR } 3.14 \\
(2.32 \text { to } \\
25)\end{array}$ & - & $\begin{array}{c}\square \square \square \\
\text { MODERATE }\end{array}$ & \\
\hline \multicolumn{13}{|c|}{ Hábito tabáquico (Holland 2013) } \\
\hline 3 & $\begin{array}{l}\text { observational } \\
\text { studies }\end{array}$ & serious $^{1}$ & $\begin{array}{l}\text { no serious } \\
\text { inconsistency }\end{array}$ & $\begin{array}{l}\text { no serious } \\
\text { indirectness }\end{array}$ & serious & none & - & - & $\begin{array}{c}\text { OR: } 1.1 \\
(0.85 \text { to } \\
1.5)\end{array}$ & - & $\begin{array}{l}\square \square \square \square \\
\text { LOW }\end{array}$ & \\
\hline \multicolumn{13}{|c|}{ Coronary artery bypass or vascular surgery (Topouzis 2011) } \\
\hline 1 & $\begin{array}{l}\text { observational } \\
\text { studies }\end{array}$ & $\begin{array}{l}\text { very } \\
\text { serious }{ }^{2}\end{array}$ & $\begin{array}{l}\text { no serious } \\
\text { inconsistency }\end{array}$ & $\begin{array}{l}\text { no serious } \\
\text { indirectness }\end{array}$ & $\begin{array}{l}\text { no serious } \\
\text { imprecision }\end{array}$ & none & $\begin{array}{l}83 / 0 \\
(0 \%)\end{array}$ & $0 \%$ & $\begin{array}{c}\text { OR } 2.09 \\
(1.16 \text { to } \\
3.77)\end{array}$ & - & $\begin{array}{c}\text { पि口ण } \\
\text { VERY LOW }\end{array}$ & \\
\hline \multicolumn{13}{|c|}{ Migraine (Holland 2013) } \\
\hline 3 & $\begin{array}{l}\text { observational } \\
\text { studies }\end{array}$ & serious $^{1}$ & $\begin{array}{l}\text { no serious } \\
\text { inconsistency }\end{array}$ & $\begin{array}{l}\text { no serious } \\
\text { indirectness }\end{array}$ & serious $^{3}$ & none & - & $0 \%$ & $\begin{array}{c}\text { OR } 0.99 \\
(0.66 \text { to } \\
1.5)\end{array}$ & - & $\begin{array}{l}\square \square \square \square \\
\text { LOW }\end{array}$ & \\
\hline
\end{tabular}

${ }^{1}$ RS observational studies included published with more than 20 years of difference. Disease definition varied over that time. In the case of family history, outcome assessed by participant slef-report of familiy history (risk of recall bias). Unclear the diabetes assessment. The included studies represented a large number of patients across many different populations with different glaucoma prevalence.

2 Temporal ambiguity of cross-sectional studies, the associations found in this study do not allow firm conclusions on risk factors for glaucoma.

${ }^{3}$ Wide $95 \%$ confidence interval. The lower boundary of the confidence interval (i.e. diabetes 1,02), could not imply any clinical intervention.

${ }^{4}$ Studies included published between 1966 and 2011. Disease definition varied over that time. The included studies represented a large number of patients across many different populations with different glaucoma prevalence. 


\section{MÉTODOS}

Como medida del efecto se utilizó un Odds Ratio con intervalo de confianza del 95\%. Como método estadístico, se usó el método de MantelHaenszel. Para el metanálisis se utilizó el modelo de efectos aleatorios.

\section{FOREST PLOTS}

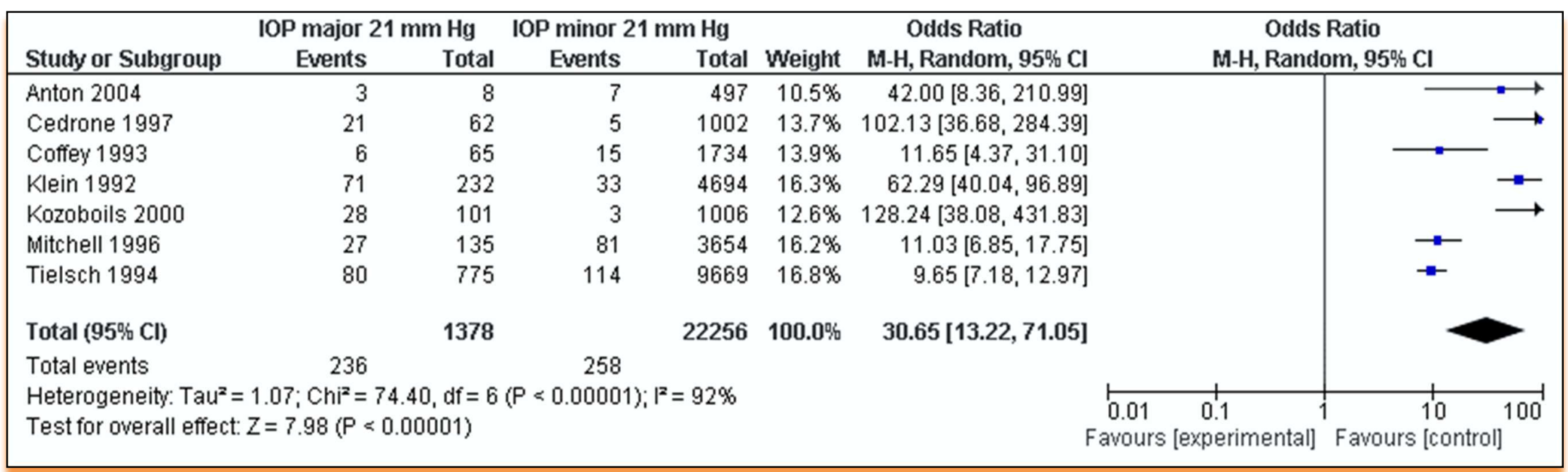




\subsubsection{Pruebas de cribado del glaucoma de ángulo abierto}

Question: Is recommended the use of ophthalmoscopy ${ }^{1}$ for glaucoma screening in the general population?

References:

Burr JM, Mowatt G, Hernández R, Siddiqui MAR, Cook J, Lourenco T et al. The clinical effectiveness and cost-effectiveness of screening for open angle glaucoma: a systematic review and economic evaluation. Health Technol Assess 2007; 11 (41).

\begin{tabular}{|c|c|c|c|c|c|c|c|c|c|c|c|}
\hline \multicolumn{7}{|c|}{ Evaluación de la calidad } & \multicolumn{3}{|c|}{$\begin{array}{c}\text { Resultados por } 1.000 \text { pacientes } \\
\text { evaluados }\end{array}$} & \multirow{2}{*}{$\begin{array}{c}\text { Calidad } \\
\text { de la } \\
\text { evidenci } \\
\text { a } \\
\text { (GRADE) }\end{array}$} & \multirow[t]{2}{*}{ Importancia } \\
\hline $\begin{array}{l}\text { Número de } \\
\text { participante } \\
\text { s (estudios) }\end{array}$ & Diseño & $\begin{array}{l}\text { Riesg } \\
\text { o de } \\
\text { sesgo }\end{array}$ & $\begin{array}{c}\text { Inconsistenci } \\
\text { a }\end{array}$ & $\begin{array}{l}\text { Evidenci } \\
\quad a \\
\text { indirecta }\end{array}$ & $\begin{array}{c}\text { Imprecisió } \\
n\end{array}$ & $\begin{array}{c}\text { Sesgo de } \\
\text { publicació } \\
\text { n }\end{array}$ & $\begin{array}{c}\text { Prevalenci } \\
\text { a } 1,4 \%^{2}\end{array}$ & $\begin{array}{c}\text { Prevalenci } \\
\text { a } 2,1 \%^{3}\end{array}$ & $\begin{array}{c}\text { Prevalenci } \\
\text { a } 6,7 \%^{4}\end{array}$ & & \\
\hline
\end{tabular}

Sensibilidad $(60 \%$; IC $95 \%=34-82)$

Verdaderos positivos (pacientes con glaucoma con resultados positivos) ${ }^{5}$

\begin{tabular}{|l|l|l|l|l|l|l|l|l|l|l|l|}
\hline $5.841(5)$ & $\begin{array}{l}\text { Cohorte } \\
\text { s } \\
\text { casos y } \\
\text { controle } \\
\mathrm{s}\end{array}$ \\
\end{tabular}




\begin{tabular}{|c|c|c|c|c|c|c|c|c|c|c|c|}
\hline $5.841(5)$ & $\begin{array}{l}\text { Cohorte } \\
\mathrm{s}+ \\
\text { casos y } \\
\text { controle } \\
\mathrm{s}\end{array}$ & Serio $^{5}$ & Serio $^{6}$ & Serio $^{7}$ & Serio $^{8}$ & $\begin{array}{l}\text { No } \\
\text { detectado }\end{array}$ & 6 & 8 & 27 & $\begin{array}{c}\square \square \square \\
\text { VERY } \\
\text { LOW }\end{array}$ & $\begin{array}{c}\text { IMPORTANT } \\
\text { E }\end{array}$ \\
\hline
\end{tabular}

Especificidad (94\%; IC 95\% = 76-99)

\section{Verdaderos negativos (pacientes sin glaucoma con resultados negativos) ${ }^{11}$}

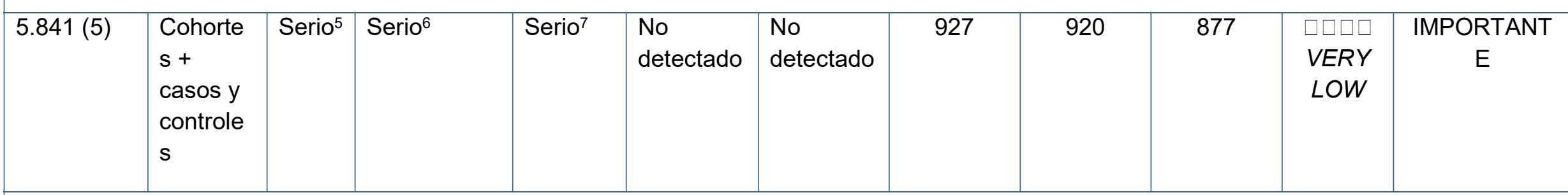

Falsos positivos (pacientes sin glaucoma con resultados positivos) ${ }^{12}$

\begin{tabular}{|c|c|c|c|c|c|c|c|c|c|c|c|}
\hline $5.841(5)$ & $\begin{array}{l}\text { Cohorte } \\
\mathrm{s}+ \\
\text { casos y } \\
\text { controle } \\
\mathrm{s}\end{array}$ & Serio 5 & Serio $^{6}$ & Serio $^{7}$ & $\begin{array}{l}\text { No } \\
\text { detectado }\end{array}$ & $\begin{array}{l}\text { No } \\
\text { detectado }\end{array}$ & 59 & 59 & 56 & $\begin{array}{l}\square \square \square \square \\
\text { VERY } \\
\text { LOW }\end{array}$ & $\begin{array}{c}\text { IMPORTANT } \\
\text { E }\end{array}$ \\
\hline
\end{tabular}

${ }^{1}$ Oftalmoscopia (disco óptico catalogado como normal o sospechoso, criterio subjetivo). Punto de corte = relación excavación-papila en diámetro vertical $(\mathrm{VCDR}) \geq 0,7$.

${ }^{2}$ Estimado por Burr (2007) para la población con 60 años $=1,4($ IC $95 \%=1,0$ a 1, 9).

${ }^{3}$ Estimado por Antón (2004) para la población general $=2,1$.

${ }^{4}$ Estimado por Burr (2007) para la población con antecedente familiar de glaucoma =6, 7 (IC 95\% = 5, 0 a 8, 4).

${ }^{5}$ Se asume que los verdaderos positivos, al clasificarse correctamente, recibirán tratamiento adecuado para su condición. Por tanto, la morbilidad asociada será menor y aumentará su calidad de vida.

${ }^{6}$ La evaluación de riesgo de sesgo por medio del instrumento QUADAS indica que, en la mayor parte de los estudios, los pacientes no fueron seguidos para confirmar el diagnóstico (estándar de referencia). Solo un estudio es calificado sin riesgo de sesgo.

${ }^{7}$ Los autores de la revisión reportan heterogeneidad tanto en las estimaciones de sensibilidad como en las de especificidad.

${ }^{8}$ En la búsqueda realizada por Burr y cols. (2007) no se identificaron estudios que permitieran evaluar el impacto de las diferentes pruebas

evaluadas en desenlaces de importancia para los pacientes. 
${ }^{9}$ El intervalo de confianza estimado para la sensibilidad es amplio; esto último puede afectar a los intervalos individuales de los verdaderos positivos y de los falsos negativos.

${ }^{10}$ Se asume que los pacientes falsos negativos, al no ser diagnosticados, no recibirían el tratamiento oportunamente.

11 Se asume que los verdaderos negativos, al ser clasificados correctamente, no recibirán pruebas o tratamientos innecesarios.

12 Se asume que los pacientes falsos positivos, al recibir el diagnóstico de glaucoma, pueden recibir pruebas complementarias y tratamientos innecesarios.

Pregunta: ¿Se recomienda el uso de la fotografía del disco óptico ${ }^{1}$ para el cribado de glaucoma en la población general?

\section{Referencias:}

Burr JM, Mowatt G, Hernández R, Siddiqui MAR, Cook J, Lourenco T et al. The clinical effectiveness and cost-effectiveness of screening for open angle glaucoma: a systematic review and economic evaluation. Health Technol Assess 2007; 11 (41).

\begin{tabular}{|c|c|c|c|c|c|c|c|c|c|c|c|}
\hline \multicolumn{7}{|c|}{ Evaluación de la calidad } & \multicolumn{3}{|c|}{$\begin{array}{c}\text { Resultados por } 1.000 \text { pacientes } \\
\text { evaluados }\end{array}$} & \multirow{2}{*}{$\begin{array}{l}\text { Calidad } \\
\text { de la } \\
\text { evidencia } \\
\text { (GRADE) }\end{array}$} & \multirow[t]{2}{*}{ Importancia } \\
\hline $\begin{array}{c}\text { Número de } \\
\text { participantes } \\
\text { (estudios) }\end{array}$ & Diseño & $\begin{array}{c}\text { Riesgo } \\
\text { de } \\
\text { sesgo }\end{array}$ & Inconsistencia & $\begin{array}{l}\text { Evidencia } \\
\text { indirecta }\end{array}$ & Imprecisión & $\begin{array}{c}\text { Sesgo de } \\
\text { publicación }\end{array}$ & $\begin{array}{c}\text { Prevalencia } \\
1,4 \%^{2}\end{array}$ & $\begin{array}{c}\text { Prevalencia } \\
2,1 \%{ }^{3}\end{array}$ & $\begin{array}{c}\text { Prevalencia } \\
6,7 \%{ }^{4}\end{array}$ & & \\
\hline
\end{tabular}

Sensibilidad $(73 \%$; IC $95 \%=61-83)$

Verdaderos positivos (pacientes con glaucoma con resultados positivos) ${ }^{5}$

\begin{tabular}{|c|c|c|c|c|c|c|c|c|c|c|c|}
\hline $7.800(6)$ & $\begin{array}{l}\text { Cohortes } \\
+ \text { casos } \\
\text { y } \\
\text { controles }\end{array}$ & Serio $^{6}$ & $\begin{array}{l}\text { No } \\
\text { detectado }\end{array}$ & Serio $^{7}$ & $\begin{array}{l}\text { No } \\
\text { detectado }\end{array}$ & $\begin{array}{l}\text { No } \\
\text { detectado }\end{array}$ & 10 & 15 & 49 & $\begin{array}{l}\square \square \square \square \\
\text { LOW }\end{array}$ & IMPORTANTE \\
\hline
\end{tabular}

\section{Falsos negativos (pacientes con glaucoma con resultados negativos) ${ }^{8}$}

\begin{tabular}{|l|l|l|l|l|l|l|l|l|l|l|}
\hline $7.800(6)$ & $\begin{array}{l}\text { Cohortes } \\
+ \text { casos } \\
\begin{array}{l}\text { controles } \\
\text { contio }\end{array}\end{array}$ & $\begin{array}{l}\text { No } \\
\text { detectado }\end{array}$ & Serio $^{7}$ & $\begin{array}{l}\text { No } \\
\text { detectado }\end{array}$ & $\begin{array}{l}\text { No } \\
\text { detectado }\end{array}$ & 4 & 6 & LOW & $\begin{array}{l}\text { IMPORTANTE } \\
\text { LOW }\end{array}$ \\
\hline
\end{tabular}




\section{Verdaderos negativos (pacientes sin glaucoma con resultados negativos) ${ }^{9}$}

\begin{tabular}{|c|c|c|c|c|c|c|c|c|c|c|c|}
\hline $7.800(6)$ & $\begin{array}{l}\text { Cohortes } \\
+ \text { casos } \\
\text { y } \\
\text { controles }\end{array}$ & Serio $^{6}$ & Serio $^{10}$ & Serio $^{7}$ & Serio $^{11}$ & $\begin{array}{l}\text { No } \\
\text { detectado }\end{array}$ & 878 & 871 & 830 & $\begin{array}{l}\square \square \square \square \\
\text { VERY } \\
\text { LOW }\end{array}$ & IMPORTANTE \\
\hline
\end{tabular}

Falsos positivos (pacientes sin glaucoma con resultados positivos) ${ }^{12}$

\begin{tabular}{|c|c|c|c|c|c|c|c|c|c|c|c|}
\hline $7.800(6)$ & $\begin{array}{l}\text { Cohortes } \\
+ \text { casos } \\
\text { y } \\
\text { controles }\end{array}$ & Serio $^{6}$ & Serio $^{10}$ & Serio $^{7}$ & Serio ${ }^{11}$ & $\begin{array}{l}\text { No } \\
\text { detectado }\end{array}$ & 108 & 108 & 103 & $\begin{array}{l}\square \square \square \square \\
\text { VERY } \\
\text { LOW }\end{array}$ & IMPORTANTE \\
\hline
\end{tabular}

${ }^{1}$ Fotografía del disco óptico (VCDR $\geq 0,7$, normal/disco glaucomatoso según la opinión de la mayoría de los observadores). Punto de corte común: $\operatorname{VCDR} \geq 0,6$.

${ }^{2}$ Estimado por Burr (2007) para la población con 60 años $=1,4$ (IC 95\% = 1, 0 a 1, 9).

${ }^{3}$ Estimado por Antón (2004) para la población general = 2, 1 .

${ }^{4}$ Estimado por Burr (2007) para la población con antecedente familiar de glaucoma $=6,7$ (IC 95\% = 5, 0 a 8, 4).

${ }^{5}$ Se asume que los verdaderos positivos, al ser clasificados correctamente, recibirán tratamiento adecuado para su condición. Por tanto, la morbilidad asociada será menor y aumentará su calidad de vida.

${ }^{6}$ La evaluación de riesgo de sesgo por medio del instrumento QUADAS indica que, en la mayor parte de los estudios, los pacientes no fueron seguidos para confirmar el diagnóstico (estándar de referencia). Dos estudios analizan un espectro de pacientes no representativo y tres son calificados de alta calidad.

${ }^{7}$ En la búsqueda realizada por Burr y cols. (2007) no se identificaron estudios que permitieran evaluar el impacto de las diferentes pruebas evaluadas en desenlaces de importancia para los pacientes.

${ }^{8}$ Se asume que los pacientes falsos negativos, al no ser diagnosticados, no recibirían el tratamiento oportunamente.

${ }^{9}$ Se asume que los verdaderos negativos, al ser clasificados correctamente, no recibirán pruebas o tratamientos innecesarios.

${ }^{10}$ Se observa considerable heterogeneidad en las cifras de especificidad proporcionadas por los estudios individuales.

${ }^{11}$ El intervalo de confianza estimado para la sensibilidad es amplio; esto último puede afectar los intervalos individuales de los verdaderos positivos y de los falsos negativos.

${ }_{12} \mathrm{Se}$ asume que los pacientes falsos positivos, al recibir el diagnóstico de glaucoma, pueden recibir pruebas complementarias y tratamientos innecesarios. 
Pregunta: ¿Se recomienda el uso de la fotografía $\mathrm{RNFL}^{1}$ para el cribado de glaucoma en la población general?

\section{Referencias:}

Burr JM, Mowatt G, Hernández R, Siddiqui MAR, Cook J, Lourenco T et al. The clinical effectiveness and cost-effectiveness of screening for open angle glaucoma: a systematic review and economic evaluation. Health Technol Assess 2007; 11 (41).

\begin{tabular}{|c|c|c|c|c|c|c|c|c|c|c|c|}
\hline \multicolumn{7}{|c|}{ Evaluación de la calidad } & \multicolumn{3}{|c|}{$\begin{array}{c}\text { Resultados por } 1.000 \text { pacientes } \\
\text { evaluados }\end{array}$} & \multirow{2}{*}{$\begin{array}{l}\text { Calidad } \\
\text { de la } \\
\text { evidencia } \\
\text { (GRADE) }\end{array}$} & \multirow[t]{2}{*}{ Importancia } \\
\hline $\begin{array}{l}\text { Número de } \\
\text { participantes } \\
\text { (estudios) }\end{array}$ & Diseño & $\begin{array}{c}\text { Riesgo } \\
\text { de } \\
\text { sesgo }\end{array}$ & Inconsistencia & $\begin{array}{l}\text { Evidencia } \\
\text { indirecta }\end{array}$ & Imprecisión & $\begin{array}{l}\text { Sesgo de } \\
\text { publicación }\end{array}$ & $\begin{array}{l}\text { Prevalencia } \\
1,4 \%^{2}\end{array}$ & $\begin{array}{l}\text { Prevalencia } \\
2,1 \%^{3}\end{array}$ & $\begin{array}{l}\text { Prevalencia } \\
\quad 6,7 \%^{4}\end{array}$ & & \\
\hline \multicolumn{12}{|c|}{ Sensibilidad $(75 \%$; IC $95 \%=46-92)$} \\
\hline \multicolumn{12}{|c|}{ Verdaderos positivos (pacientes con glaucoma con resultados positivos) $^{5}$} \\
\hline \multicolumn{12}{|c|}{ Falsos negativos (pacientes con glaucoma con resultados negativos) ${ }^{9}$} \\
\hline \multicolumn{12}{|c|}{ Especificidad $(88 \%$; IC $95 \%=53-98)$} \\
\hline \multicolumn{12}{|c|}{ Verdaderos negativos (pacientes sin glaucoma con resultados negativos) ${ }^{10}$} \\
\hline $700(4)$ & $\begin{array}{l}\text { Cohortes } \\
+ \text { casos } \\
y\end{array}$ & Serio 6 & $\begin{array}{l}\text { No } \\
\text { detectado }\end{array}$ & Serio $^{7}$ & Serio $^{8}$ & $\begin{array}{l}\text { No } \\
\text { detectado }\end{array}$ & 868 & 862 & 821 & $\begin{array}{l}\square \square \square \square \\
\text { VERY }\end{array}$ & IMPORTANTE \\
\hline
\end{tabular}




\begin{tabular}{|l|l|l|l|l|l|l|l|l|l|l|}
\hline & controles & & & & & LOW & \\
\hline
\end{tabular}

${ }^{1}$ Punto de corte común: pérdida de la capa de fibras nerviosas.

${ }^{2}$ Estimado por Burr (2007) para la población con 60 años $=1,4$ (IC 95\% = 1, 0 a 1, 9).

${ }^{3}$ Estimado por Antón (2004) para la población general $=2,1$.

${ }^{4}$ Estimado por Burr (2007) para la población con antecedente familiar de glaucoma $=6,7$ (IC 95\% = 5, 0 a 8, 4).

${ }^{5}$ Se asume que los verdaderos positivos, al ser clasificados correctamente, recibirán tratamiento adecuado para su condición. Por tanto, la morbilidad asociada será menor y aumentará su calidad de vida.

${ }^{6}$ La evaluación de riesgo de sesgo por medio del instrumento QUADAS indica que, en la mayor parte de los estudios, los pacientes no fueron seguidos para confirmar el diagnóstico (estándar de referencia). Ningún estudio es calificado como de alta calidad.

${ }^{7}$ En la búsqueda realizada por Burr y cols. (2007) no se identificaron estudios que permitieran evaluar el impacto de las diferentes pruebas evaluadas en desenlaces de importancia para los pacientes.

${ }^{8}$ El intervalo de confianza estimado para la sensibilidad y la especificidad es amplio; esto último puede afectar a los intervalos individuales de todas las estimaciones de verdaderos y falsos positivos y negativos.

${ }^{9}$ Se asume que los pacientes falsos negativos, al no ser diagnosticados, no recibirían el tratamiento oportunamente.

${ }^{10}$ Se asume que los verdaderos negativos, al ser clasificados correctamente, no recibirán pruebas o tratamientos innecesarios.

${ }^{11}$ Se asume que los pacientes falsos positivos, al recibir el diagnóstico de glaucoma, pueden recibir pruebas complementarias y tratamientos innecesarios.

Pregunta: ¿Se recomienda el uso de la HRT II ${ }^{1}$ para el cribado de glaucoma en la población general?

\section{Referencias:}

Burr JM, Mowatt G, Hernández R, Siddiqui MAR, Cook J, Lourenco T et al. The clinical effectiveness and cost-effectiveness of screening for open angle glaucoma: a systematic review and economic evaluation. Health Technol Assess 2007; 11 (41).

\begin{tabular}{|c|c|c|c|}
\hline Evaluación de la calidad & Resultados por 1.000 pacientes & Calidad & Importancia \\
\hline
\end{tabular}




\begin{tabular}{|c|c|c|c|c|c|c|c|c|c|c|}
\hline & & & & & & & & evaluados & & evidencia \\
\hline $\begin{array}{c}\text { Número de } \\
\text { participantes } \\
\text { (estudios) }\end{array}$ & Diseño & $\begin{array}{c}\text { Riesgo } \\
\text { de } \\
\text { sesgo }\end{array}$ & Inconsistencia & $\begin{array}{l}\text { Evidencia } \\
\text { indirecta }\end{array}$ & Imprecisión & $\begin{array}{c}\text { Sesgo de } \\
\text { publicación }\end{array}$ & $\begin{array}{c}\text { Prevalencia } \\
1,4 \%^{2}\end{array}$ & $\begin{array}{c}\text { Prevalencia } \\
2,1 \%^{3}\end{array}$ & $\begin{array}{c}\text { Prevalencia } \\
6,7 \%^{4}\end{array}$ & \\
\hline
\end{tabular}

Sensibilidad $(86 \%$; IC $95 \%=55-97)$

Verdaderos positivos (pacientes con glaucoma con resultados positivos) ${ }^{5}$

\begin{tabular}{|c|c|c|c|c|c|c|c|c|c|c|c|}
\hline $1.073(3)$ & $\begin{array}{l}\text { Cohortes } \\
+ \text { casos } \\
\text { y } \\
\text { controles }\end{array}$ & Serio $^{6}$ & $\begin{array}{l}\text { No } \\
\text { detectado }\end{array}$ & Serio $^{7}$ & Serio $^{8}$ & $\begin{array}{l}\text { No } \\
\text { detectado }\end{array}$ & 12 & 18 & 58 & 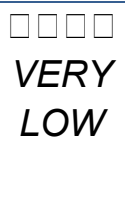 & IMPORTANTE \\
\hline
\end{tabular}

Falsos negativos (pacientes con glaucoma con resultados negativos) ${ }^{9}$

\begin{tabular}{|c|c|c|c|c|c|c|c|c|c|c|c|}
\hline $700(4)$ & $\begin{array}{l}\text { Cohortes } \\
+ \text { casos } \\
\text { y } \\
\text { controles }\end{array}$ & Serio $^{6}$ & $\begin{array}{l}\text { No } \\
\text { detectado }\end{array}$ & Serio $^{7}$ & Serio $^{8}$ & $\begin{array}{l}\text { No } \\
\text { detectado }\end{array}$ & 2 & 3 & 9 & $\begin{array}{l}\text { VER } \\
\text { VERY } \\
\text { LOW }\end{array}$ & IMPORTANTE \\
\hline
\end{tabular}

Especificidad $(89 \%$; IC 95\% $=66-98)$

Verdaderos negativos (pacientes sin glaucoma con resultados negativos) ${ }^{10}$

\begin{tabular}{|c|c|c|c|c|c|c|c|c|c|c|c|}
\hline $700(4)$ & $\begin{array}{l}\text { Cohortes } \\
+ \text { casos } \\
\text { y } \\
\text { controles }\end{array}$ & Serio ${ }^{6}$ & $\begin{array}{l}\text { No } \\
\text { detectado }\end{array}$ & Serio $^{7}$ & Serio $^{8}$ & $\begin{array}{l}\text { No } \\
\text { detectado }\end{array}$ & 878 & 871 & 830 & $\begin{array}{l}\text { VIDQ } \\
\text { VERY } \\
\text { LOW }\end{array}$ & IMPORTANTE \\
\hline
\end{tabular}

Falsos positivos (pacientes sin glaucoma con resultados positivos) ${ }^{11}$

\begin{tabular}{|c|c|c|c|c|c|c|c|c|c|c|c|}
\hline $700(4)$ & $\begin{array}{l}\text { Cohortes } \\
+ \text { casos } \\
\text { y } \\
\text { controles }\end{array}$ & Serio 6 & $\begin{array}{l}\text { No } \\
\text { detectado }\end{array}$ & Serio $^{7}$ & Serio $^{8}$ & $\begin{array}{l}\text { No } \\
\text { detectado }\end{array}$ & 108 & 108 & 103 & $\begin{array}{l}\text { VERY } \\
\text { LOW }\end{array}$ & IMPORTANTE \\
\hline
\end{tabular}


${ }^{1}$ Punto de corte común: $\geq 1$ límite o fuera de los límites normales.

2 Estimado por Burr (2007) para la población con 60 años = 1, 4 (IC 95\% = 1, 0 a 1, 9).

${ }^{3}$ Estimado por Antón (2004) para la población general = 2, 1 .

${ }^{4}$ Estimado por Burr (2007) para la población con antecedente familiar de glaucoma = 6, 7 (IC 95\% = 5, 0 a 8, 4).

${ }^{5} \mathrm{Se}$ asume que los verdaderos positivos, al ser clasificados correctamente, recibirán tratamiento adecuado para su condición. Por tanto, la morbilidad asociada será menor y aumentará su calidad de vida.

${ }^{6}$ La evaluación de riesgo de sesgo por medio del instrumento QUADAS indica que, en la mayor parte de los estudios, los pacientes no fueron seguidos para confirmar el diagnóstico (estándar de referencia).

${ }^{7}$ En la búsqueda realizada por Burr y cols. (2007) no se identificaron estudios que permitieran evaluar el impacto de las diferentes pruebas evaluadas en desenlaces de importancia para los pacientes.

${ }^{8}$ El intervalo de confianza estimado para la sensibilidad y la especificidad es amplio; esto último puede afectar a los intervalos individuales de todas las estimaciones de verdaderos y falsos positivos y negativos.

${ }^{9} \mathrm{Se}$ asume que los pacientes falsos negativos, al no ser diagnosticados, no recibirían el tratamiento oportunamente.

${ }^{10} \mathrm{Se}$ asume que los verdaderos negativos, al ser clasificados correctamente, no recibirán pruebas o tratamientos innecesarios.

${ }^{11}$ Se asume que los pacientes falsos positivos, al recibir el diagnóstico de glaucoma, pueden recibir pruebas complementarias y tratamientos innecesarios.

Pregunta: ¿Se recomienda el uso de la FDT (C-20-1 $)^{1}$ para el cribado de glaucoma en la población general?

\section{Referencias:}

Burr JM, Mowatt G, Hernández R, Siddiqui MAR, Cook J, Lourenco T et al. The clinical effectiveness and cost-effectiveness of screening for open angle glaucoma: a systematic review and economic evaluation. Health Technol Assess $2007 ; 11$ (41).

\begin{tabular}{|c|c|c|c|c|c|c|c|c|c|c|c|}
\hline \multicolumn{7}{|c|}{ Evaluación de la calidad } & \multicolumn{3}{|c|}{$\begin{array}{c}\text { Resultados por } 1.000 \text { pacientes } \\
\text { evaluados }\end{array}$} & \multirow{2}{*}{$\begin{array}{l}\text { Calidad de } \\
\text { la evidencia } \\
\text { (GRADE) }\end{array}$} & \multirow[t]{2}{*}{ Importancia } \\
\hline $\begin{array}{l}\text { Número de } \\
\text { participante } \\
\text { s (estudios) }\end{array}$ & Diseño & $\begin{array}{l}\text { Riesg } \\
\text { o de } \\
\text { sesgo }\end{array}$ & $\begin{array}{c}\text { Inconsistenci } \\
\text { a }\end{array}$ & $\begin{array}{c}\text { Evidenci } \\
\text { a } \\
\text { indirecta }\end{array}$ & Imprecisión & $\begin{array}{l}\text { Sesgo de } \\
\text { publicación }\end{array}$ & $\begin{array}{l}\text { Prevalenci } \\
\text { a } 1,4 \%^{2}\end{array}$ & $\begin{array}{l}\text { Prevalenci } \\
\text { a } 2,1 \%^{3}\end{array}$ & $\begin{array}{c}\text { Prevale } \\
\text { ncia } 6 \\
7 \% 4\end{array}$ & & \\
\hline \multicolumn{12}{|c|}{ Sensibilidad (92\%; IC 95\% = 65-99) } \\
\hline \multicolumn{12}{|c|}{ Verdaderos positivos (pacientes con glaucoma con resultados positivos) 5} \\
\hline $575(3)$ & $\begin{array}{l}\text { Cohorte } \\
\mathrm{s}+ \\
\text { casos y }\end{array}$ & $\begin{array}{l}\text { Serio } \\
6\end{array}$ & $\begin{array}{l}\text { No } \\
\text { detectado }\end{array}$ & Serio $^{7}$ & Serio $^{8}$ & No detectado & 13 & 19 & 62 & $\begin{array}{l}\square \square \square \square \\
\text { VERY }\end{array}$ & IMPORTANTE \\
\hline
\end{tabular}




\begin{tabular}{|c|c|c|c|c|c|c|c|c|c|c|c|c|c|c|}
\hline & $\begin{array}{l}\text { controle } \\
\mathrm{s}\end{array}$ & & & & & & & & & & & & LOW & \\
\hline \multicolumn{15}{|c|}{ Falsos negativos (pacientes con glaucoma con resultados negativos) ${ }^{9}$} \\
\hline \multicolumn{15}{|c|}{ Especificidad (94\%; IC 95\% = 73-99) } \\
\hline \multicolumn{15}{|c|}{ Verdaderos negativos (pacientes sin glaucoma con resultados negativos) ${ }^{10}$} \\
\hline $575(3)$ & $\begin{array}{l}\text { Cohorte } \\
\text { s }+ \\
\text { casos y } \\
\text { controle } \\
\text { s }\end{array}$ & $\begin{array}{l}\text { Serio } \\
6\end{array}$ & Serio $^{11}$ & Serio $^{7}$ & $\begin{array}{l}\text { No } \\
\text { detect }\end{array}$ & & Noc & etectado & 927 & 92 & 20 & 877 & $\begin{array}{l}\text { VID } \\
\text { VERY } \\
\text { LOW }\end{array}$ & IMPORTANTE \\
\hline \multicolumn{15}{|c|}{ Falsos positivos (pacientes sin glaucoma con resultados positivos) ${ }^{12}$} \\
\hline $575(3)$ & $\begin{array}{l}\text { Cohortes } \\
\text { casos y } \\
\text { controles }\end{array}$ & & Serio $^{6}$ & Serio $^{11}$ & $\begin{array}{l}\text { Serio } \\
7\end{array}$ & $\begin{array}{l}\text { No } \\
\text { dete } \\
\text { o }\end{array}$ & tad & $\begin{array}{l}\text { No } \\
\text { detectad } \\
0\end{array}$ & 59 & 59 & 56 & $\begin{array}{c}\square \square \\
\square \\
\text { VERY } \\
\text { LOW }\end{array}$ & \multicolumn{2}{|c|}{ IMPORTANTE } \\
\hline
\end{tabular}

${ }^{1}$ Punto de corte común: 1 punto anormal.

${ }^{2}$ Estimado por Burr (2007) para la población con 60 años $=1,4$ (IC 95\% = 1, 0 a 1, 9).

${ }^{3}$ Estimado por Antón (2004) para la población general = 2, 1 .

${ }^{4}$ Estimado por Burr (2007) para la población con antecedente familiar de glaucoma $=6,7($ IC 95\% = 5, 0 a 8, 4).

${ }^{5}$ Se asume que los verdaderos positivos, al ser clasificados correctamente, recibirán tratamiento adecuado para su condición. Por tanto, la morbilidad asociada será menor y aumentará su calidad de vida.

${ }^{6}$ La evaluación de riesgo de sesgo por medio del instrumento QUADAS indica que, en la mayor parte de los estudios, los pacientes no fueron seguidos para confirmar el diagnóstico (estándar de referencia). 
${ }^{7}$ En la búsqueda realizada por Burr y cols. (2007) no se identificaron estudios que permitieran evaluar el impacto de las diferentes pruebas evaluadas en desenlaces de importancia para los pacientes.

${ }^{8}$ El intervalo de confianza estimado para la sensibilidad y la especificidad es amplio; esto último puede afectar los intervalos individuales de todas las estimaciones de verdaderos y falsos positivos y negativos.

${ }^{9}$ Se asume que los pacientes falsos negativos, al no ser diagnosticados, no recibirían el tratamiento oportunamente.

${ }^{10}$ Se asume que los verdaderos negativos, al ser clasificados correctamente, no recibirán pruebas o tratamientos innecesarios.

${ }^{11}$ Se observa considerable heterogeneidad en las cifras de especificidad proporcionadas por los estudios individuales.

${ }^{12}$ Se asume que los pacientes falsos positivos, al recibir el diagnóstico de glaucoma, pueden recibir pruebas complementarias y tratamientos innecesarios.

Pregunta: ¿Se recomienda el uso de la FDT $(\mathrm{C}-20-5)^{1}$ para el cribado de glaucoma en la población general?

Referencias:

Burr JM, Mowatt G, Hernández R, Siddiqui MAR, Cook J, Lourenco T et al. The clinical effectiveness and cost-effectiveness of screening for open angle glaucoma: a systematic review and economic evaluation. Health Technol Assess 2007; 11 (41).

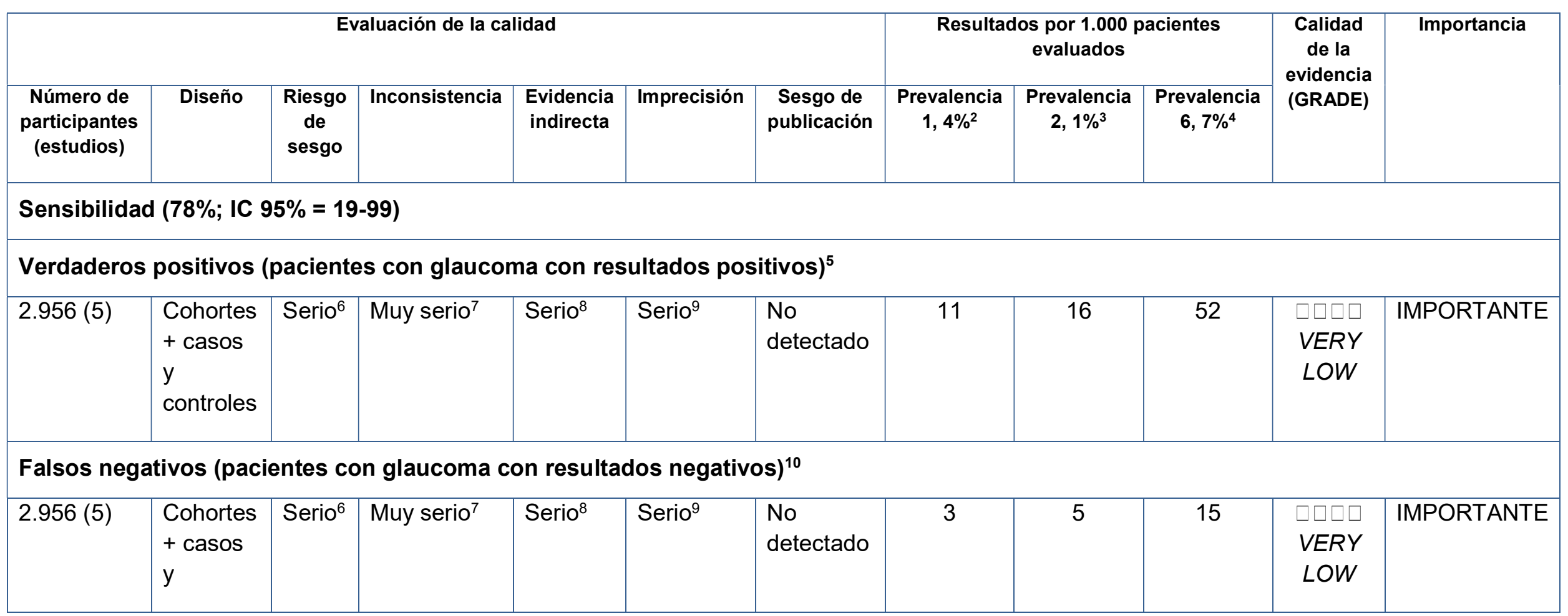




\begin{tabular}{|l|l|l|l|l|l|l|l|l|l|l|}
\hline & controles & & & & & \\
\hline
\end{tabular}

${ }^{1}$ Punto de corte: 1 punto anormal.

${ }^{2}$ Estimado por Burr (2007) para la población con 60 años $=1,4($ IC $95 \%=1,0$ a 1, 9).

${ }^{3}$ Estimado por Antón (2004) para la población general $=2,1$.

${ }^{4}$ Estimado por Burr (2007) para la población con antecedente familiar de glaucoma $=6,7$ (IC 95\% = 5, 0 a 8, 4).

${ }^{5}$ Se asume que los verdaderos positivos, al ser clasificados correctamente, recibirán tratamiento adecuado para su condición. Por tanto, la morbilidad asociada será menor y aumentará su calidad de vida.

${ }^{6}$ La evaluación de riesgo de sesgo por medio del instrumento QUADAS indica que, en la mayor parte de los estudios, los pacientes no fueron seguidos para confirmar el diagnóstico (estándar de referencia).Solo dos estudios fueron clasificados como de alta calidad.

${ }^{7}$ Se observa alta heterogeneidad en las cifras de sensibilidad y especificidad proporcionadas por los estudios individuales.

${ }^{8}$ En la búsqueda realizada por Burr y cols. (2007) no se identificaron estudios que permitieran evaluar el impacto de las diferentes pruebas evaluadas en desenlaces de importancia para los pacientes.

${ }^{9} \mathrm{El}$ intervalo de confianza estimado para la sensibilidad es amplio; esto último puede afectar los intervalos individuales de los verdaderos positivos y de los falsos negativos.

${ }_{10}^{10}$ Se asume que los pacientes falsos negativos, al no ser diagnosticados, no recibirían el tratamiento oportunamente.

${ }^{11}$ Se asume que los verdaderos negativos, al ser clasificados correctamente, pueden tener un riesgo menor de complicaciones futuras asociadas a su salud visual.

${ }^{12}$ Se asume que los pacientes falsos positivos, al recibir el diagnóstico de glaucoma, pueden recibir pruebas complementarias y tratamientos innecesarios. 
Pregunta: ¿Se recomienda el uso de la $\mathrm{OKP}^{1}$ para el cribado de glaucoma en la población general?

\section{Referencias:}

Burr JM, Mowatt G, Hernández R, Siddiqui MAR, Cook J, Lourenco T et al. The clinical effectiveness and cost-effectiveness of screening for open angle glaucoma: a systematic review and economic evaluation. Health Technol Assess 2007; 11 (41).

\begin{tabular}{|c|c|c|c|c|c|c|c|c|c|c|c|}
\hline \multicolumn{7}{|c|}{ Evaluación de la calidad } & \multicolumn{3}{|c|}{$\begin{array}{c}\text { Resultados por } 1.000 \text { pacientes } \\
\text { evaluados }\end{array}$} & \multirow{2}{*}{$\begin{array}{l}\text { Calidad } \\
\text { de la } \\
\text { evidencia } \\
\text { (GRADE) }\end{array}$} & \multirow[t]{2}{*}{ Importancia } \\
\hline $\begin{array}{l}\text { Número de } \\
\text { participantes } \\
\text { (estudios) }\end{array}$ & Diseño & $\begin{array}{l}\text { Riesgo } \\
\text { de } \\
\text { sesgo }\end{array}$ & Inconsistencia & $\begin{array}{l}\text { Evidencia } \\
\text { indirecta }\end{array}$ & Imprecisión & $\begin{array}{c}\text { Sesgo de } \\
\text { publicación }\end{array}$ & $\begin{array}{c}\text { Prevalencia } \\
1,4 \%{ }^{2}\end{array}$ & $\begin{array}{l}\text { Prevalencia } \\
2,1 \%{ }^{3}\end{array}$ & $\begin{array}{c}\text { Prevalencia } \\
6,7 \% 4\end{array}$ & & \\
\hline
\end{tabular}

\section{Sensibilidad $(86 \%$; IC $95 \%=29-100)$}

\section{Verdaderos positivos (pacientes con glaucoma con resultados positivos) ${ }^{5}$}

\begin{tabular}{|c|c|c|c|c|c|c|c|c|c|c|c|}
\hline $768(4)$ & $\begin{array}{l}\text { Cohortes } \\
+ \text { casos } \\
\text { y } \\
\text { controles }\end{array}$ & Serio 6 & Serio $^{7}$ & Serio $^{8}$ & Serio $^{9}$ & $\begin{array}{l}\text { No } \\
\text { detectado }\end{array}$ & 12 & 18 & 58 & $\begin{array}{c}\square \square \square \square \\
\text { VERY } \\
\text { LOW }\end{array}$ & IMPORTANTE \\
\hline
\end{tabular}

Falsos negativos (pacientes con glaucoma con resultados negativos) ${ }^{10}$

\begin{tabular}{|c|c|c|c|c|c|c|c|c|c|c|c|}
\hline $768(4)$ & $\begin{array}{l}\text { Cohortes } \\
+ \text { casos } \\
\text { y } \\
\text { controles }\end{array}$ & Serio $^{6}$ & Serio $^{7}$ & Serio $^{8}$ & Serio $^{9}$ & $\begin{array}{l}\text { No } \\
\text { detectado }\end{array}$ & 2 & 3 & 9 & $\begin{array}{l}\square \square \square \square \\
\text { VERY } \\
\text { LOW }\end{array}$ & IMPORTANTE \\
\hline
\end{tabular}

Especificidad (90\%; IC 95\% = 79-96)

Verdaderos negativos (pacientes sin glaucoma con resultados negativos) ${ }^{11}$

\begin{tabular}{|c|c|c|c|c|c|c|c|c|c|c|c|}
\hline $768(4)$ & $\begin{array}{l}\text { Cohortes } \\
+ \text { casos } \\
\text { y } \\
\text { controles }\end{array}$ & Serio $^{6}$ & Serio $^{7}$ & Serio $^{8}$ & $\begin{array}{l}\text { No } \\
\text { detectado }\end{array}$ & $\begin{array}{l}\text { No } \\
\text { detectado }\end{array}$ & 887 & 881 & 840 & $\begin{array}{l}\text { VERY } \\
\text { LOW }\end{array}$ & IMPORTANTE \\
\hline
\end{tabular}


Falsos positivos (pacientes sin glaucoma con resultados positivos) ${ }^{12}$

\begin{tabular}{|c|c|c|c|c|c|c|c|c|c|c|c|}
\hline $768(4)$ & $\begin{array}{l}\text { Cohortes } \\
+ \text { casos } \\
\text { y } \\
\text { controles }\end{array}$ & Serio $^{6}$ & Muy serio ${ }^{7}$ & Serio $^{8}$ & $\begin{array}{l}\text { No } \\
\text { detectado }\end{array}$ & $\begin{array}{l}\text { No } \\
\text { detectado }\end{array}$ & 99 & 98 & 93 & $\begin{array}{l}\square \square \square \square \\
\text { VERY } \\
\text { LOW }\end{array}$ & IMPORTANTE \\
\hline
\end{tabular}

${ }^{1}$ Punto de corte: 1 punto anormal.

${ }^{2}$ Estimado por Burr (2007) para la población con 60 años $=1,4$ (IC 95\% = 1, 0 a 1, 9).

${ }^{3}$ Estimado por Antón (2004) para la población general $=2,1$.

${ }^{4}$ Estimado por Burr (2007) para la población con antecedente familiar de glaucoma =6, 7 (IC 95\% = 5, 0 a 8, 4).

${ }^{5}$ Se asume que los verdaderos positivos, al ser clasificados correctamente, recibirán tratamiento adecuado para su condición. Por tanto, la morbilidad asociada será menor y aumentará su calidad de vida.

${ }^{6} \mathrm{La}$ evaluación de riesgo de sesgo por medio del instrumento QUADAS indica que, en la mayor parte de los estudios, los pacientes no fueron seguidos para confirmar el diagnóstico (estándar de referencia). Solo un estudio fue clasificado como de alta calidad.

${ }^{7}$ Se observa alta heterogeneidad en las cifras de sensibilidad y especificidad proporcionadas por los estudios individuales.

${ }^{8}$ En la búsqueda realizada por Burr y cols. (2007) no se identificaron estudios que permitieran evaluar el impacto de las diferentes pruebas evaluadas en desenlaces de importancia para los pacientes.

${ }^{9} \mathrm{El}$ intervalo de confianza estimado para la sensibilidad es amplio; esto último puede afectar los intervalos individuales de los verdaderos positivos y de los falsos negativos.

${ }_{10} \mathrm{Se}$ asume que los pacientes falsos negativos, al no ser diagnosticados, no recibirían el tratamiento oportunamente.

${ }^{11}$ Se asume que los verdaderos negativos, al ser clasificados correctamente, no recibirán pruebas o tratamientos innecesarios.

${ }^{12} \mathrm{Se}$ asume que los pacientes falsos positivos, al recibir el diagnóstico de glaucoma, pueden recibir pruebas complementarias y tratamientos innecesarios.

Pregunta: ¿Se recomienda el uso de la SAP supraumbral ${ }^{1}$ para el cribado de glaucoma en la población general?

\section{Referencias:}

Burr JM, Mowatt G, Hernández R, Siddiqui MAR, Cook J, Lourenco T et al. The clinical effectiveness and cost-effectiveness of screening for open angle glaucoma: a systematic review and economic evaluation. Health Technol Assess 2007; 11 (41).

\begin{tabular}{|c|c|c|c|c|c|c|c|c|c|c|c|}
\hline \multicolumn{7}{|c|}{ Evaluación de la calidad } & \multicolumn{3}{|c|}{$\begin{array}{c}\text { Resultados por } 1.000 \text { pacientes } \\
\text { evaluados }\end{array}$} & \multirow{2}{*}{$\begin{array}{l}\text { Calidad } \\
\text { de la } \\
\text { evidencia } \\
\text { (GRADE) }\end{array}$} & \multirow[t]{2}{*}{ Importancia } \\
\hline $\begin{array}{l}\text { Número de } \\
\text { participantes }\end{array}$ & Diseño & $\begin{array}{c}\text { Riesgo } \\
\text { de }\end{array}$ & Inconsistencia & $\begin{array}{l}\text { Evidencia } \\
\text { indirecta }\end{array}$ & Imprecisión & $\begin{array}{l}\text { Sesgo de } \\
\text { publicación }\end{array}$ & $\begin{array}{c}\text { Prevalencia } \\
1,4 \%^{2}\end{array}$ & $\begin{array}{c}\text { Prevalencia } \\
2,1 \%^{3}\end{array}$ & $\begin{array}{c}\text { Prevalencia } \\
6,7 \%^{4}\end{array}$ & & \\
\hline
\end{tabular}




\begin{tabular}{|c|c|c|c|c|c|c|c|c|c|c|c|}
\hline (estudios) & & sesgo & & & & & & & & & \\
\hline \multicolumn{12}{|c|}{ Sensibilidad $(71 \%$; IC $95 \%=51-86)$} \\
\hline $10.200(9)$ & $\begin{array}{l}\text { Cohortes } \\
+ \text { casos } \\
\text { y } \\
\text { controles }\end{array}$ & Serio $^{6}$ & Serio $^{7}$ & Serio $^{8}$ & Serio $^{9}$ & $\begin{array}{l}\text { No } \\
\text { detectado }\end{array}$ & 10 & 15 & 48 & $\begin{array}{l}\square \square \square \square \\
\text { VERY } \\
\text { LOW }\end{array}$ & IMPORTANTE \\
\hline \multicolumn{12}{|c|}{ Falsos negativos (pacientes con glaucoma con resultados negativos) ${ }^{10}$} \\
\hline $10.200(9)$ & $\begin{array}{l}\text { Cohortes } \\
+ \text { casos } \\
\text { y } \\
\text { controles }\end{array}$ & Serio $^{6}$ & Serio $^{7}$ & Serio $^{8}$ & Serio $^{9}$ & $\begin{array}{l}\text { No } \\
\text { detectado }\end{array}$ & 4 & 6 & 19 & $\begin{array}{l}\text { VERY } \\
\text { LOW }\end{array}$ & IMPORTANTE \\
\hline $10.200(9)$ & $\begin{array}{l}\text { Cohortes } \\
+ \text { casos } \\
\text { y } \\
\text { controles }\end{array}$ & Serio $^{6}$ & Serio $^{7}$ & Serio $^{8}$ & $\begin{array}{l}\text { No } \\
\text { detectado }\end{array}$ & $\begin{array}{l}\text { No } \\
\text { detectado }\end{array}$ & 838 & 832 & 793 & $\begin{array}{l}\text { VERY } \\
\text { LOW }\end{array}$ & IMPORTANTE \\
\hline \multicolumn{12}{|c|}{ Falsos positivos (pacientes sin glaucoma con resultados positivos) ${ }^{12}$} \\
\hline $10.200(9)$ & $\begin{array}{l}\text { Cohortes } \\
+ \text { casos } \\
\text { y } \\
\text { controles }\end{array}$ & Serio $^{6}$ & Muy serio ${ }^{7}$ & Serio $^{8}$ & $\begin{array}{l}\text { No } \\
\text { detectado }\end{array}$ & $\begin{array}{l}\text { No } \\
\text { detectado }\end{array}$ & 148 & 147 & 140 & $\begin{array}{l}\square \square \square \square \\
\text { VERY } \\
\text { LOW }\end{array}$ & IMPORTANTE \\
\hline
\end{tabular}


${ }_{1}^{1}$ Punto de corte común: $\geq 3$ points missing. $\geq 17$ relative or absolute defects and/or cluster of 8 in any one quadrant, $\geq 4$ abnormal points in any single quadrant, sufficient points to drop the indicator into the suspicious zone or below, 3 abnormal adjacent points, $\geq 1$ missed point optometrist judgment, at least 1 absolute defect associated with 1 relative defect or 3 adjacent relative defects or 4 non-adjacent relative defects or sure nasal step.

Estimado por Burr (2007) para la población con 60 años $=1,4$ (IC 95\% = 1, 0 a 1, 9).

${ }^{3}$ Estimado por Antón (2004) para la población general = 2, 1 .

${ }^{4}$ Estimado por Burr (2007) para la población con antecedente familiar de glaucoma $=6,7$ (IC 95\% = 5, 0 a 8, 4).

${ }^{5}$ Se asume que los verdaderos positivos, al ser clasificados correctamente, recibirán tratamiento adecuado para su condición. Por tanto, la morbilidad asociada será menor y aumentará su calidad de vida.

${ }^{6}$ La evaluación de riesgo de sesgo por medio del instrumento QUADAS indica que, en un gran número de estudios, los pacientes no

fueron seguidos para confirmar el diagnóstico (estándar de referencia), así como la presencia de sesgo de incorporación. Solo un estudio fue clasificado como de alta calidad.

7 Se observa alta heterogeneidad en las cifras de sensibilidad y especificidad proporcionadas por los estudios individuales, la cual no es explicada por el diseño o por los participantes.

${ }^{8}$ En la búsqueda realizada por Burr y cols. (2007) no se identificaron estudios que permitieran evaluar el impacto de las diferentes pruebas evaluadas en desenlaces de importancia para los pacientes.

${ }^{9} \mathrm{El}$ intervalo de confianza estimado para la sensibilidad es amplio; esto último puede afectar los intervalos individuales de los verdaderos positivos y de los falsos negativos.

${ }^{10}$ Se asume que los pacientes falsos negativos, al no ser diagnosticados, no recibirían el tratamiento oportunamente.

${ }^{11}$ Se asume que los verdaderos negativos, al ser clasificados correctamente, no recibirán pruebas o tratamientos innecesarios.

${ }^{12} \mathrm{Se}$ asume que los pacientes falsos positivos, al recibir el diagnóstico de glaucoma, pueden recibir pruebas complementarias y tratamientos innecesarios. 
Pregunta: ¿Se recomienda el uso de la SAP umbral ${ }^{1}$ para el cribado de glaucoma en la población general? Referencias:

Burr JM, Mowatt G, Hernández R, Siddiqui MAR, Cook J, Lourenco T, et al. The clinical effectiveness and cost-effectiveness of screening for open angle glaucoma: a systematic review and economic evaluation. Health Technol Assess 2007; 11 (41).

\begin{tabular}{|c|c|c|c|c|c|c|c|c|c|c|c|}
\hline \multicolumn{7}{|c|}{ Evaluación de la calidad } & \multicolumn{3}{|c|}{$\begin{array}{l}\text { Resultados por } 1.000 \text { pacientes } \\
\text { evaluados }\end{array}$} & \multirow{2}{*}{$\begin{array}{c}\text { Calidad } \\
\text { de la } \\
\text { evidenci } \\
\text { a } \\
\text { (GRADE) }\end{array}$} & \multirow[t]{2}{*}{ Importancia } \\
\hline $\begin{array}{l}\text { Número de } \\
\text { participante } \\
\text { s (estudios) }\end{array}$ & Diseño & $\begin{array}{l}\text { Riesg } \\
\text { o de } \\
\text { sesgo }\end{array}$ & $\begin{array}{c}\text { Inconsistenci } \\
\text { a }\end{array}$ & $\begin{array}{c}\text { Evidenci } \\
\text { a } \\
\text { indirecta }\end{array}$ & $\begin{array}{c}\text { Imprecisió } \\
\mathrm{n}\end{array}$ & $\begin{array}{c}\text { Sesgo de } \\
\text { publicació } \\
n\end{array}$ & $\begin{array}{c}\text { Prevalenci } \\
\text { a } 1,4 \%^{2}\end{array}$ & $\begin{array}{l}\text { Prevalenci } \\
\text { a } 2,1 \%^{3}\end{array}$ & $\begin{array}{l}\text { Prevalenci } \\
\text { a } 6,7 \%^{4}\end{array}$ & & \\
\hline
\end{tabular}

Sensibilidad $(88 \%$; IC 95\% = 65-97)

Verdaderos positivos (pacientes con glaucoma con resultados positivos)

\begin{tabular}{|l|l|l|l|l|l|l|l|l|l|l|l|}
\hline $1.457(5)$ & $\begin{array}{l}\text { Cohorte } \\
\mathrm{s}+ \\
\text { casos y } \\
\text { controle } \\
\mathrm{s}\end{array}$ & $\begin{array}{l}\text { Serio } \\
\mathrm{G}\end{array}$ & Serio $^{7}$ & Serio $^{8}$ & Serio $^{9}$ & $\begin{array}{l}\text { No } \\
\text { detectad } \\
0\end{array}$ & 12 & 18 & 59 & $\begin{array}{l}\text { पD口 } \\
\text { VERY } \\
\text { LOW }\end{array}$ & $\begin{array}{l}\text { IMPORTANT } \\
\text { E }\end{array}$ \\
\hline
\end{tabular}

Falsos negativos (pacientes con glaucoma con resultados negativos) ${ }^{10}$

\begin{tabular}{|c|c|c|c|c|c|c|c|c|c|c|c|}
\hline $1.457(5)$ & $\begin{array}{l}\text { Cohorte } \\
\mathrm{s}+ \\
\text { casos y } \\
\text { controle } \\
\mathrm{s}\end{array}$ & \begin{tabular}{|l} 
Serio \\
6
\end{tabular} & Serio $^{7}$ & Serio $^{8}$ & Serio $^{9}$ & $\begin{array}{l}\text { No } \\
\text { detectad } \\
\text { o }\end{array}$ & 2 & 3 & 8 & $\begin{array}{l}\text { VEDG } \\
\text { VERY } \\
\text { LOW }\end{array}$ & $\begin{array}{c}\text { IMPORTANT } \\
\text { E }\end{array}$ \\
\hline \multicolumn{12}{|c|}{ Especificidad $(80 \%$; IC $95 \%=55-93)$} \\
\hline \multicolumn{12}{|c|}{ Verdaderos negativos (pacientes sin glaucoma con resultados negativos) ${ }^{11}$} \\
\hline $1.457(5)$ & $\begin{array}{l}\text { Cohorte } \\
\mathrm{s}+\end{array}$ & Serio & Serio $^{7}$ & Serio $^{8}$ & Serio $^{9}$ & \begin{tabular}{|l|} 
No \\
detectad
\end{tabular} & 789 & 783 & 746 & $\begin{array}{l}\text { पिमा } \\
\text { VERY }\end{array}$ & IMPORTANT \\
\hline
\end{tabular}




\begin{tabular}{|c|c|c|c|c|c|c|c|c|c|c|c|}
\hline & $\begin{array}{l}\text { casos y } \\
\text { controle } \\
\text { s }\end{array}$ & 6 & & & & 0 & & & & LOW & $\mathrm{E}$ \\
\hline \multicolumn{12}{|c|}{ Falsos positivos (pacientes sin glaucoma con resultados positivos) ${ }^{12}$} \\
\hline $1.457(5)$ & $\begin{array}{l}\text { Cohorte } \\
\mathrm{s}+ \\
\text { casos y } \\
\text { controle } \\
\mathrm{s}\end{array}$ & $\begin{array}{l}\text { Serio } \\
6\end{array}$ & Muy serio ${ }^{7}$ & Serio $^{8}$ & Serio $^{9}$ & $\begin{array}{l}\text { No } \\
\text { detectad } \\
\text { o }\end{array}$ & 197 & 196 & 187 & $\begin{array}{l}\text { पिGप } \\
\text { VERY } \\
\text { LOW }\end{array}$ & $\begin{array}{c}\text { IMPORTANT } \\
\text { E }\end{array}$ \\
\hline
\end{tabular}

${ }^{1}$ Punto de corte común: AGIS score $\geq 3$. Cross meridional, GHT abnormal/borderline, LDA 59 points, mirror image method, GHT outside normal limit and/or PSD $P<0.05$ in one or both eyes.

Estimado por Burr (2007) para la población con 60 años = 1, 4 (IC 95\% = 1, 0 a 1, 9)

${ }^{3}$ Estimado por Antón (2004) para la población general = 2, 1.

${ }^{4}$ Estimado por Burr (2007) para la población con antecedente familiar de glaucoma $=6,7$ (IC 95\% = 5, 0 a 8, 4).

${ }^{5}$ Se asume que los verdaderos positivos, al ser clasificados correctamente, recibirán tratamiento adecuado para su condición. Por tanto, la morbilidad asociada será menor y aumentará su calidad de vida.

${ }^{6}$ La evaluación de riesgo de sesgo por medio del instrumento QUADAS indica que, en un gran número de estudios, los pacientes no fueron seguidos para confirmar el diagnóstico (estándar de referencia), así como la presencia de sesgo de incorporación. Solo un estudio fue clasificado como de alta calidad.

${ }^{7}$ Se observa alta heterogeneidad en las cifras de sensibilidad y especificidad proporcionadas por los estudios individuales, la cual no es explicada por el diseño o por los participantes.

${ }^{8}$ En la búsqueda realizada por Burr y cols. (2007) no se identificaron estudios que permitieran evaluar el impacto de las diferentes pruebas evaluadas en desenlaces de importancia para los pacientes.

${ }^{9}$ El intervalo de confianza estimado para la sensibilidad y la especificidad es amplio; esto último puede afectar los intervalos individuales de los verdaderos y falsos negativos y positivos.

${ }^{10}$ Se asume que los pacientes falsos negativos, al no ser diagnosticados, no recibirían el tratamiento oportunamente.

${ }^{11}$ Se asume que los verdaderos negativos, al ser clasificados correctamente, no recibirán pruebas o tratamientos innecesarios.

${ }^{12} \mathrm{Se}$ asume que los pacientes falsos positivos, al recibir el diagnóstico de glaucoma, pueden recibir pruebas complementarias y tratamientos innecesarios. 
Pregunta: ¿Se recomienda el uso de la GAT ${ }^{1}$ para el cribado de glaucoma en la población general?

\section{Referencias:}

Burr JM, Mowatt G, Hernández R, Siddiqui MAR, Cook J, Lourenco T, et al. The clinical effectiveness and cost-effectiveness of screening for open angle glaucoma: a systematic review and economic evaluation. Health Technol Assess 2007; 11 (41).

\begin{tabular}{|c|c|c|c|c|c|c|c|c|c|c|c|}
\hline \multicolumn{7}{|c|}{ Evaluación de la calidad } & \multicolumn{3}{|c|}{$\begin{array}{l}\text { Resultados por } 1.000 \text { pacientes } \\
\text { evaluados }\end{array}$} & \multirow{2}{*}{$\begin{array}{c}\text { Calidad } \\
\text { de la } \\
\text { evidenci } \\
\text { a } \\
\text { (GRADE) }\end{array}$} & \multirow[t]{2}{*}{ Importancia } \\
\hline $\begin{array}{l}\text { Número de } \\
\text { participante } \\
\text { s (estudios) }\end{array}$ & Diseño & $\begin{array}{l}\text { Riesg } \\
\text { o de } \\
\text { sesgo }\end{array}$ & $\begin{array}{c}\text { Inconsistenci } \\
\text { a }\end{array}$ & $\begin{array}{l}\text { Evidenci } \\
\text { a } \\
\text { indirecta }\end{array}$ & $\begin{array}{c}\text { Imprecisió } \\
n\end{array}$ & $\begin{array}{c}\text { Sesgo de } \\
\text { publicació } \\
\text { n }\end{array}$ & $\begin{array}{l}\text { Prevalenci } \\
\text { a } 1,4 \%^{2}\end{array}$ & $\begin{array}{l}\text { Prevalenci } \\
\text { a 2, } 1 \%^{3}\end{array}$ & $\begin{array}{l}\text { Prevalenci } \\
\text { a } 6,7 \%^{4}\end{array}$ & & \\
\hline
\end{tabular}

Sensibilidad $(46 \%$; IC $95 \%=22-71)$

Verdaderos positivos (pacientes con glaucoma con resultados positivos)

\begin{tabular}{|c|c|c|c|c|c|c|c|c|c|}
\hline $20.308(9)$ & $\begin{array}{l}\text { Cohorte } \\
\mathrm{s}+ \\
\text { casos y } \\
\text { controle } \\
\mathrm{s}\end{array}$ & $\begin{array}{l}\text { Serio } \\
6\end{array}$ & Serio $^{7}$ & Serio $^{8}$ & Serio $^{9}$ & $\begin{array}{l}\text { No } \\
\text { detectad } \\
\text { o }\end{array}$ & 6 & 1 & $\begin{array}{l}\text { IMPORTANT } \\
\text { E }\end{array}$ \\
\hline
\end{tabular}

Falsos negativos (pacientes con glaucoma con resultados negativos) ${ }^{10}$

\begin{tabular}{|c|c|c|c|c|c|c|c|c|c|c|c|}
\hline $20.308(9)$ & $\begin{array}{l}\text { Cohorte } \\
\mathrm{s}+ \\
\text { casos y } \\
\text { controle } \\
\mathrm{s}\end{array}$ & $\begin{array}{l}\text { Serio } \\
6\end{array}$ & Serio $^{7}$ & Serio $^{8}$ & Serio $^{9}$ & \begin{tabular}{|l|} 
No \\
detectad \\
o
\end{tabular} & 8 & 11 & 36 & $\begin{array}{l}\text { पDGD } \\
\text { VERY } \\
\text { LOW }\end{array}$ & $\begin{array}{c}\text { IMPORTANT } \\
\text { E }\end{array}$ \\
\hline \multicolumn{12}{|c|}{ Especificidad (95\%; IC 95\% = 88-97) } \\
\hline \multicolumn{12}{|c|}{ Verdaderos negativos (pacientes sin glaucoma con resultados negativos) ${ }^{11}$} \\
\hline $20.308(9)$ & $\begin{array}{l}\text { Cohorte } \\
\mathrm{s}+\end{array}$ & Serio & Serio $^{7}$ & Serio $^{8}$ & \begin{tabular}{|l} 
No \\
detectad
\end{tabular} & \begin{tabular}{|l} 
No \\
detectad
\end{tabular} & 937 & 930 & 886 & $\begin{array}{l}\text { प्रिप } \\
\text { VERY }\end{array}$ & IMPORTANT \\
\hline
\end{tabular}




\begin{tabular}{|c|c|c|c|c|c|c|c|c|c|c|c|}
\hline & $\begin{array}{l}\text { casos y } \\
\text { controle } \\
\text { s }\end{array}$ & 6 & & & 0 & 0 & & & & LOW & $\mathrm{E}$ \\
\hline \multicolumn{12}{|c|}{ Falsos positivos (pacientes sin glaucoma con resultados positivos) ${ }^{12}$} \\
\hline $20.308(9)$ & $\begin{array}{l}\text { Cohorte } \\
\mathrm{s}+ \\
\text { casos y } \\
\text { controle } \\
\mathrm{s}\end{array}$ & \begin{tabular}{|l|} 
Serio \\
6
\end{tabular} & Muy serio ${ }^{7}$ & Serio $^{8}$ & \begin{tabular}{|l|} 
No \\
detectad \\
o
\end{tabular} & \begin{tabular}{|l|} 
No \\
detectad \\
o
\end{tabular} & 49 & 49 & 47 & $\begin{array}{l}\text { पि口प } \\
\text { VERY } \\
\text { LOW }\end{array}$ & $\begin{array}{c}\text { IMPORTANT } \\
\text { E }\end{array}$ \\
\hline
\end{tabular}

${ }^{1}$ Punto de corte común: PIO $\geq 21 \mathrm{mmHg}$, IOP $21-22 \mathrm{mmHg}$, PIO $>22 \mathrm{mmHg}$.

${ }^{2}$ Estimado por Burr (2007) para la población con 60 años = 1, 4 (IC 95\% = 1, 0 a 1, 9).

${ }^{3}$ Estimado por Antón (2004) para la población general = 2, 1.

${ }^{4}$ Estimado por Burr (2007) para la población con antecedente familiar de glaucoma = 6, 7 (IC 95\% = 5, 0 a 8, 4).

${ }^{5}$ Se asume que los verdaderos positivos, al ser clasificados correctamente, recibirán tratamiento adecuado para su condición. Por tanto, la morbilidad asociada será menor y aumentará su calidad de vida.

${ }^{6}$ La evaluación de riesgo de sesgo por medio del instrumento QUADAS indica que, en un gran número de estudios, los pacientes no

fueron seguidos para confirmar el diagnóstico (estándar de referencia), así como la presencia de sesgo de verificación diferencial y sesgo de diagnóstico al conocer el resultado de la prueba de referencia. Ningún estudio fue clasificado como de alta calidad.

${ }^{7}$ Se observa alta heterogeneidad en las cifras de sensibilidad y especificidad proporcionadas por los estudios individuales, la cual no es explicada por el diseño ni por los participantes.

${ }^{8}$ En la búsqueda realizada por Burr y cols. (2007) no se identificaron estudios que permitieran evaluar el impacto de las diferentes pruebas evaluadas en desenlaces de importancia para los pacientes.

${ }^{9}$ El intervalo de confianza estimado para la sensibilidad y la especificidad es amplio; esto último puede afectar los intervalos individuales de os verdaderos y falsos negativos y positivos.

${ }^{10}$ Se asume que los pacientes falsos negativos, al no ser diagnosticados, no recibirían el tratamiento oportunamente.

${ }^{11} \mathrm{Se}$ asume que los verdaderos negativos, al ser clasificados correctamente, no recibirán pruebas o tratamientos innecesarios.

${ }^{12} \mathrm{Se}$ asume que los pacientes falsos positivos, al recibir el diagnóstico de glaucoma, pueden recibir pruebas complementarias y tratamientos innecesarios. 
Pregunta: ¿Se recomienda el cribado poblacional de glaucoma para prevenir daños en el nervio óptico?

\section{Referencia:}

Hatt SR, Wormald R, Burr J. Screening for prevention of optic nerve damage due to chronic open angle glaucoma. Cochrane Database of Systematic Reviews 2006, Issue 4. Art. No.: CD006129. DOI: 10.1002/14651858.CD006129.pub2.

\begin{tabular}{|c|c|c|c|c|c|c|c|c|c|c|c|c|}
\hline \multicolumn{7}{|c|}{ Quality assessment } & \multicolumn{2}{|c|}{ No of patients } & \multicolumn{2}{|c|}{ Effect } & \multirow{2}{*}{ Quality } & \multirow{2}{*}{ Importance } \\
\hline $\begin{array}{l}\text { No of } \\
\text { studies }\end{array}$ & Design & $\begin{array}{l}\text { Risk of } \\
\text { bias }\end{array}$ & Inconsistency & Indirectness & Imprecision & $\begin{array}{c}\text { Other } \\
\text { considerations }\end{array}$ & Screening & $\begin{array}{c}\text { No } \\
\text { screening }\end{array}$ & $\begin{array}{c}\text { Relative } \\
\text { (95\% } \\
\text { Cl) }\end{array}$ & Absolute & & \\
\hline
\end{tabular}

Prevalence of any degree of characteristic visual field loss in screened and non-screened populations (diagnosed by any automated or manual visual field assessment)

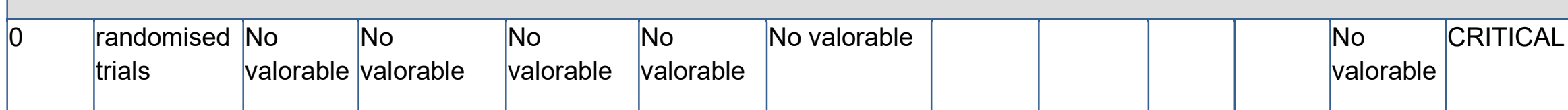

Prevalence of optic nerve damage in screened and non-screened populations (diagnosed by any method of imaging).

\begin{tabular}{|l|l|l|l|l|l|l|l|l|l|l|l|}
\hline 0 & $\begin{array}{l}\text { randomised } \\
\text { trials }\end{array}$ & $\begin{array}{l}\text { No } \\
\text { valorable }\end{array}$ & $\begin{array}{l}\text { No } \\
\text { valorable }\end{array}$ & $\begin{array}{l}\text { No } \\
\text { valorable }\end{array}$ & $\begin{array}{l}\text { No } \\
\text { valorable }\end{array}$ & No valorable & & & & & $\begin{array}{l}\text { No } \\
\text { valorable }\end{array}$ \\
\hline
\end{tabular}

Prevalence of visual impairment in screened and non-screened populations (defined by number of participants certified or registered according to national or regional standards)

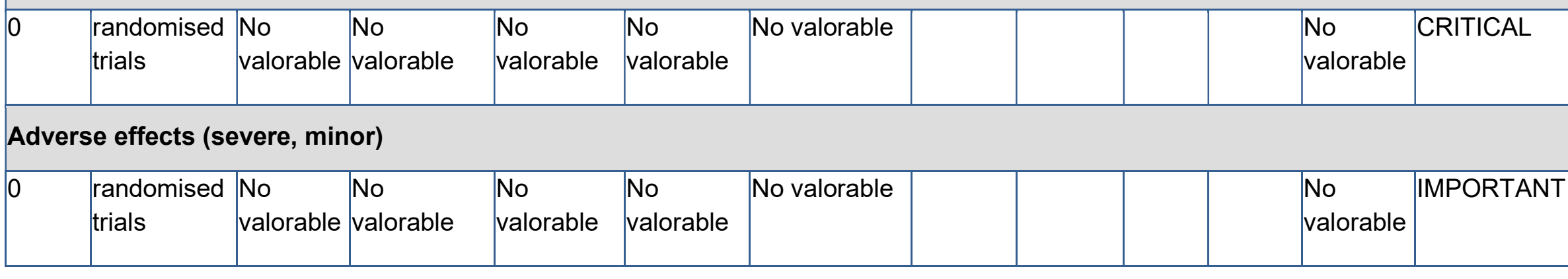


Quality of life measures

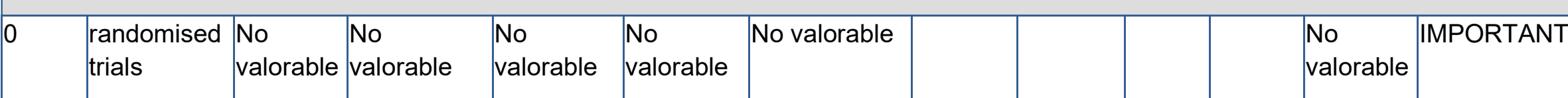




\subsection{Tratamiento farmacológico del glaucoma de ángulo abierto}

4.2.1. Opciones farmacológicas en el tratamiento del glaucoma de ángulo abierto

4.2.1.1. Parasimpaticomiméticos frente betabloqueadores

Question: Should parasimpaticomimetics vs beta-blockers be used for primary open angle glaucoma and ocular hypertension? Bibliography:

Commissioned by the National Institute for Health and Clinical Excellence. Glaucoma Diagnosis and management of chronic open angle glaucoma and ocular hypertension. UK. 2009.

\begin{tabular}{|c|c|c|c|c|c|c|c|c|c|c|c|c|}
\hline \multicolumn{7}{|c|}{ Quality assessment } & \multicolumn{2}{|l|}{ No of patients } & \multicolumn{2}{|c|}{ Effect } & \multirow{2}{*}{ Quality } & \multirow{2}{*}{ Importance } \\
\hline \begin{tabular}{|} 
No of \\
studies
\end{tabular} & Design & $\begin{array}{c}\text { Risk of } \\
\text { bias }\end{array}$ & Inconsistency & Indirectness & Imprecision & $\begin{array}{c}\text { Other } \\
\text { considerations }\end{array}$ & Parasimpaticomimetics & $\begin{array}{c}\text { Beta- } \\
\text { blockers }\end{array}$ & $\begin{array}{c}\text { Relative } \\
\text { (95\% } \\
\text { Cl) }\end{array}$ & Absolute & & \\
\hline \multicolumn{13}{|c|}{ Mean change in IOP from baseline (follow up 17 to 24 months) (better indicated by lower values) } \\
\hline 3 & $\begin{array}{l}\text { randomised } \\
\text { trials }\end{array}$ & serious $^{1}$ & $\begin{array}{l}\text { no serious } \\
\text { inconsistency }\end{array}$ & serious ${ }^{2}$ & \begin{tabular}{|l|} 
no serious \\
imprecision
\end{tabular} & none & 102 & 73 & - & \begin{tabular}{c|} 
not \\
pooled
\end{tabular} & $\begin{array}{l}\text { Lिवर् } \\
\text { LOW }\end{array}$ & CRITICAL \\
\hline \multicolumn{13}{|c|}{ Mild adverses events } \\
\hline 1 & $\begin{array}{l}\text { observationa } \\
\text { studies }\end{array}$ & | serious ${ }^{3}$ & $\begin{array}{l}\text { no serious } \\
\text { inconsistency }\end{array}$ & $\begin{array}{l}\text { no serious } \\
\text { indirectness }\end{array}$ & serious ${ }^{4}$ & none & $\begin{array}{c}4 /- \\
(0 \%)\end{array}$ & $\begin{array}{c}12 /- \\
(0 \%)\end{array}$ & $\begin{array}{c}\text { not } \\
\text { pooled }\end{array}$ & \begin{tabular}{c|} 
not \\
pooled
\end{tabular} & \begin{tabular}{|l|} 
पिएव \\
VERY \\
LOW
\end{tabular} & CRITICAL \\
\hline
\end{tabular}

${ }^{1}$ Methods of randomisation is not described and there is no mention of allocation concealment. 
2 OAG progression as inferred from IOP.

${ }^{3}$ The same eye could be included in more than one group when the treatment was changed.

${ }^{4}$ Very small sample size.

4.2.1.2. Inhibidores de la anhidrasa carbónica frente a betabloqueadores

Question: Should carbonic anhydrase inhibitors vs beta-blockers be used for primary open angle glaucoma and ocular hypertension? Bibliography:

Commissioned by the National Institute for Health and Clinical Excellence. Glaucoma Diagnosis and management of chronic open angle glaucoma and ocular hypertension. UK. 2009.

\begin{tabular}{|c|c|c|c|c|c|c|c|c|c|c|c|c|}
\hline \multicolumn{7}{|c|}{ Quality assessment } & \multicolumn{2}{|c|}{ No of patients } & \multicolumn{2}{|c|}{ Effect } & \multirow{2}{*}{ Quality } & \multirow{2}{*}{ Importance } \\
\hline $\begin{array}{c}\text { No of } \\
\text { studies }\end{array}$ & Design & $\begin{array}{l}\text { Risk of } \\
\text { bias }\end{array}$ & Inconsistency & Indirectness & Imprecision & $\begin{array}{c}\text { Other } \\
\text { considerations }\end{array}$ & $\begin{array}{c}\text { Carbonic } \\
\text { anhydrase } \\
\text { inhibitors }\end{array}$ & $\begin{array}{c}\text { Beta- } \\
\text { blockers }\end{array}$ & $\begin{array}{l}\text { Relative } \\
(95 \% \mathrm{Cl})\end{array}$ & Absolute & & \\
\hline \multicolumn{13}{|c|}{ Mean change in IOP from baseline (follow up 12 to 18 months) (better indicated by lower values) } \\
\hline 2 & \begin{tabular}{|l} 
randomised \\
trials
\end{tabular} & d serious 1 , & $\begin{array}{l}\text { no serious } \\
\text { inconsistency }\end{array}$ & serious $^{3}$ & \begin{tabular}{|l} 
no serious \\
imprecision
\end{tabular} & none & 463 & 178 & - & $\begin{array}{c}\text { not } \\
\text { pooled }\end{array}$ & $\begin{array}{l}\text { पिएव } \\
\text { LOW }\end{array}$ & CRITICAL \\
\hline \multicolumn{13}{|c|}{ Number of patients with hyperaemia } \\
\hline 1 & $\begin{array}{l}\text { randomised } \\
\text { trials }\end{array}$ & d serious 1 & $\begin{array}{l}\text { no serious } \\
\text { inconsistency }\end{array}$ & $\begin{array}{l}\text { no serious } \\
\text { indirectness }\end{array}$ & serious $^{4}$ & none & $\begin{array}{l}4 / 150 \\
(2.7 \%)\end{array}$ & $\begin{array}{l}0 / 75 \\
(0 \%)\end{array}$ & $\begin{array}{c}\text { RR } 4.53 \\
(0.25 \text { to } \\
83.05)\end{array}$ & - & $\begin{array}{l}\text { पिएव } \\
\text { LOW }\end{array}$ & CRITICAL \\
\hline
\end{tabular}

${ }^{1}$ Not reported how patients were randomised or it there was allocation concealment.

${ }^{2}$ Outcomes not resported properly. One study does not report the standard deviations associated with the mean reductions, nor the IOP at the end of the study.

${ }^{3}$ OAG progression as inferred from IOP.

${ }^{4}$ Wide intervals of confidence. 
4.2.1.3. Análogos de las prostaglandinas frente a betabloqueadores

Question: Should prostaglandin analogues vs beta-blockers be used for primary open angle glaucoma and ocular hypertension? Bibliography:

Commissioned by the National Institute for Health and Clinical Excellence. Glaucoma Diagnosis and management of chronic open angle glaucoma and ocular hypertension. UK. 2009.

\begin{tabular}{|c|c|c|c|c|c|c|c|c|c|c|c|c|}
\hline \multicolumn{7}{|c|}{ Quality assessment } & \multicolumn{2}{|c|}{ No of patients } & \multicolumn{2}{|c|}{ Effect } & \multirow{2}{*}{ Quality } & \multirow{2}{*}{ Importance } \\
\hline \begin{tabular}{|c|} 
No of \\
studies
\end{tabular} & Design & $\begin{array}{c}\text { Risk of } \\
\text { bias }\end{array}$ & Inconsistency & Indirectness & Imprecision & $\begin{array}{c}\text { Other } \\
\text { considerations }\end{array}$ & $\begin{array}{c}\text { Prostaglandin } \\
\text { analogues }\end{array}$ & $\begin{array}{l}\text { Beta- } \\
\text { blockers }\end{array}$ & \begin{tabular}{|c|} 
Relative \\
$(95 \%$ \\
Cl)
\end{tabular} & Absolute & & \\
\hline
\end{tabular}

Mean change in IOP from baseline (follow up 6 to 36 months) (better indicated by lower values)

\begin{tabular}{|c|c|c|c|c|c|c|c|c|c|c|c|}
\hline 12 & $\begin{array}{l}\text { randomised } \\
\text { trials }\end{array}$ & serious $^{2}$ & serious $^{3}$ & $\mid$\begin{tabular}{|l|} 
no serious \\
imprecision
\end{tabular} & none & 1342 & 1.333 & - & \begin{tabular}{|c|}
$\mathrm{MD}$ \\
1.32 \\
lower \\
$(1.79$ to \\
0.84 \\
lower $)^{4}$
\end{tabular} & $\begin{array}{c}\text { पिएव } \\
\text { VERY LOW }\end{array}$ & CRITICAL \\
\hline \multicolumn{12}{|c|}{ Number of patients with an acceptable IOP (follow up 6 to 12 months) } \\
\hline 7 & $\begin{array}{l}\mid \begin{array}{l}\text { randomised } \\
\text { trials }\end{array} \\
\text { serious } 1 \\
\end{array}$ & serious $^{2}$ & serious $^{3}$ & \begin{tabular}{|l|} 
no serious \\
imprecision
\end{tabular} & none & $\begin{array}{l}546 / 971 \\
(56.2 \%)\end{array}$ & $\begin{array}{l}376 / 953 \\
(39.5 \%)\end{array}$ & $\begin{array}{c}\mathrm{RR} \\
1.54 \\
(1.21 \\
\text { to } \\
1.96)\end{array}$ & \begin{tabular}{|c|}
213 \\
more \\
per \\
1.000 \\
(from \\
83 \\
more to \\
379
\end{tabular} & $\begin{array}{c}\text { पिएव } \\
\text { VERY LOW }\end{array}$ & CRITICAL \\
\hline
\end{tabular}




\begin{tabular}{|l|l|l|l|l|l|l|l|l|l|l|}
\hline & & & & & & & & \\
\hline
\end{tabular}




\begin{tabular}{|l|l|l|l|l|l|l|l|l|l|l|}
\hline & & & & & more) & \\
\hline
\end{tabular}

${ }_{1}^{1}$ Randomisation method and alocation concealment were no reported in some of the studies. ${ }^{2}$ Significant heterogeneity found in overall result. No specific cause for heterogeneity identified. ${ }^{3}$ OAG progression as inferred from IOP.

Theasured by $\mathrm{mm} \mathrm{Hg}$.

The confidence intervals are wide making the estimate of harm uncertain.

\subsubsection{Análogos de prostaglandinas frente a inhibidor de la anhidrasa carbónica}

Question: Should latonoprost vs dorzolamide be used for primary open angle glaucoma and ocular hypertension? Bibliography:

Hodge WG, Lachaine J, Steffensen I, Murray C, Barnes D, Foerster V, Ducruet T, Morrison A. The efficacy and harm of prostaglandin analogues for IOP reduction in glaucoma patients compared to dorzolamide and brimonidine: a systematic review. $\mathrm{Br} \mathrm{J}$ Ophthalmol. 2008; 92(1): 7-12. 


\begin{tabular}{|c|c|c|c|c|c|c|c|c|c|c|c|c|}
\hline \multicolumn{7}{|c|}{ Quality assessment } & \multicolumn{2}{|c|}{ No of patients } & \multicolumn{2}{|c|}{ Effect } & \multirow{2}{*}{ Quality } & \multirow{2}{*}{ Importance } \\
\hline \begin{tabular}{|c|} 
No of \\
studies
\end{tabular} & Design & \begin{tabular}{|c|} 
Risk of \\
bias
\end{tabular} & Inconsistency & Indirectness & Imprecision & $\begin{array}{c}\text { Other } \\
\text { considerations }\end{array}$ & Latonoprost & Dorzolamide & \begin{tabular}{|l|} 
Relative \\
$(95 \% \mathrm{Cl})$
\end{tabular} & Absolute & & \\
\hline \multicolumn{13}{|c|}{ Weighted mean difference of IOP (better indicated by lower values) } \\
\hline 3 & $\begin{array}{l}\text { randomised } \\
\text { trials }\end{array}$ & serious $^{1}$ & $\begin{array}{l}\text { no serious } \\
\text { inconsistency }\end{array}$ & serious $^{2}$ & serious $^{3}$ & none & 164 & 164 & - & $\begin{array}{c}\text { MD } 2.64 \\
\text { lower } \\
(3.25 \text { to } \\
2.04 \\
\text { lower) }\end{array}$ & \begin{tabular}{|l|l|} 
VERT \\
LERY \\
\end{tabular} & CRITICAL \\
\hline \multicolumn{13}{|c|}{ Withdrawals due to adverse events } \\
\hline 2 & $\begin{array}{l}\text { randomised } \\
\text { trials }\end{array}$ & serious $^{1}$ & $\begin{array}{l}\text { no serious } \\
\text { inconsistency } 5\end{array}$ & serious $^{6}$ & serious $^{7}$ & none & $\begin{array}{c}1 / 127 \\
(0.79 \%)\end{array}$ & $\begin{array}{c}1 / 127 \\
(0.79 \%)\end{array}$ & \begin{tabular}{|c|}
$R R$ \\
1.00 \\
$(0.06$ to \\
$15.79)$
\end{tabular} & \begin{tabular}{|c|}
0 fewer \\
per 1.000 \\
(from 7 \\
fewer to \\
116 \\
more)
\end{tabular} & \begin{tabular}{|l|l|} 
VERम \\
VERY \\
LOW
\end{tabular} & CRITICAL \\
\hline \multicolumn{13}{|c|}{ Ocular hyperaemia } \\
\hline 4 & $\begin{array}{l}\text { randomised } \\
\text { trials }\end{array}$ & serious $^{1}$ & $\begin{array}{l}\text { no serious } \\
\text { inconsistency }\end{array}$ & $\begin{array}{l}\text { no serious } \\
\text { indirectness }\end{array}$ & serious & none & $\begin{array}{l}13 / 179 \\
(7.3 \%)\end{array}$ & $\begin{array}{l}11 / 179 \\
(6.1 \%)\end{array}$ & \begin{tabular}{|c|} 
RR \\
1.18 \\
$(0.59$ to \\
$2.37)$ \\
\end{tabular} & $\begin{array}{c}11 \text { more } \\
\text { per } 1.000 \\
\text { (from } 25 \\
\text { fewer to } \\
84 \text { more) }\end{array}$ & \begin{tabular}{|l|} 
LOU \\
LOW
\end{tabular} & CRITICAL \\
\hline \multicolumn{13}{|c|}{ All other ocular adverse events (excluding hyperemia) } \\
\hline 4 & $\begin{array}{l}\text { randomised } \\
\text { trials }\end{array}$ & serious $^{1}$ & $\begin{array}{l}\text { no serious } \\
\text { inconsistency }\end{array}$ & $\begin{array}{l}\text { no serious } \\
\text { indirectness }\end{array}$ & serious $^{7}$ & none & $\begin{array}{c}73 / 179 \\
(40.8 \%)\end{array}$ & $\begin{array}{c}80 / 179 \\
(44.7 \%)\end{array}$ & $\begin{array}{l}R R \\
0.91\end{array}$ & \begin{tabular}{|l|}
40 fewer \\
per 1.000
\end{tabular} & \begin{tabular}{|l|}
$\square \square \square \square$ \\
LOW
\end{tabular} & CRITICAL \\
\hline
\end{tabular}




\begin{tabular}{|c|c|c|c|c|c|c|c|c|c|c|c|c|}
\hline & & & & & & & & & \begin{tabular}{|c|}
$(0.73$ to \\
$1.14)$
\end{tabular} & $\mid \begin{array}{c}\text { (from 121 } \\
\text { fewer to } \\
63 \text { more) }\end{array}$ & & \\
\hline \multicolumn{13}{|c|}{ Serious adverses events } \\
\hline 3 & $\begin{array}{l}\text { randomised } \\
\text { trials }\end{array}$ & serious $^{1}$ & $\begin{array}{l}1 \text { no serious } \\
\text { inconsistency }\end{array}$ & \begin{tabular}{|l|} 
no serious \\
indirectness
\end{tabular} & serious $^{7}$ & none & $\begin{array}{l}3 / 157 \\
(1.9 \%)\end{array}$ & $\begin{array}{l}3 / 157 \\
(1.9 \%)\end{array}$ & \begin{tabular}{|c}
$\mathrm{RR}$ \\
1.00 \\
$(0.21$ to \\
$4.85)$
\end{tabular} & $\begin{array}{c}0 \text { fewer } \\
\text { per } 1.000 \\
\text { (from } 15 \\
\text { fewer to } \\
74 \text { more) }\end{array}$ & $\begin{array}{l}\text { पिएव } \\
\text { LOW }\end{array}$ & CRITICAL \\
\hline
\end{tabular}

1 Jadad score $=2$, The studies did not specify methods of randomization, allocation concealment or blinding

2 OAG progression as inferred from IOP.

${ }^{3}$ Total population size is less than 400

${ }^{4}$ Measured by $\mathrm{mm} \mathrm{Hg}$.

${ }^{5}$ No data provided.

${ }^{6}$ This outcome does not directly measure the adverses effects.

${ }^{7}$ Low number of events. $95 \% \mathrm{Cl}$ does not exclude appreciable benefit or harm.

4.2.1.5. Betabloqueadores frente a agonistas alfa selectivos

Question: Should timolol vs. brimonidine be used for primary open angle glaucoma and ocular hypertension [Data only. When citing this record quote "Cochrane Database of Systematic Reviews 2007, Issue 4".]?

\section{Bibliography:}

LoonSC, Liew G, Fung A, Reid SE, Craig JC. Meta-analysis of randomized controlled trials comparing timolol with brimonidine in the treatment of glaucoma. Clin Experiment Ophthalmol. 2008; 36(3): 281-9.

Krupin T, Liebmann JM, Greenfield DS, Ritch R, Gardiner S; Low-Pressure Glaucoma Study Group. A randomized trial of brimonidine versus timolol in preserving visual function: results from the Low-Pressure Glaucoma Treatment Study. Am J Ophthalmol. 2011 Apr; 151 (4): 671-81. 


\begin{tabular}{|c|c|c|c|c|c|c|c|c|c|c|c|c|}
\hline \multicolumn{7}{|c|}{ Quality assessment } & \multicolumn{2}{|c|}{ No of patients } & \multicolumn{2}{|c|}{ Effect } & \multirow{2}{*}{ Quality } & \multirow{2}{*}{ Importance } \\
\hline \begin{tabular}{|c|}
$\begin{array}{c}\text { No of } \\
\text { studies }\end{array}$ \\
\end{tabular} & Design & $\begin{array}{c}\text { Risk of } \\
\text { bias }\end{array}$ & Inconsistency & Indirectness & Imprecision & $\begin{array}{c}\text { Other } \\
\text { considerations }\end{array}$ & Timolol & Brimonidine & $\begin{array}{l}\text { Relative } \\
(95 \% \mathrm{Cl})\end{array}$ & Absolute & & \\
\hline
\end{tabular}

Visual field progression (defect) (assessed with: visual field progression in either eye as determined by with progressor software (Medisoft Inc., Leeds, UK).)

\begin{tabular}{|c|c|c|c|c|c|c|c|c|c|c|c|c|}
\hline 11 & \begin{tabular}{|l} 
randomised \\
trials
\end{tabular} & $\begin{array}{l}\text { no } \\
\text { serious } \\
\text { risk of } \\
\text { bias }\end{array}$ & $\begin{array}{l}\text { no serious } \\
\text { inconsistency }\end{array}$ & no serious & $\begin{array}{l}\text { very } \\
\text { serious }^{2}\end{array}$ & none & $\begin{array}{c}9 / 99 \\
(9.1 \%)\end{array}$ & $\begin{array}{c}31 / 79 \\
(39.2 \%)\end{array}$ & \begin{tabular}{|c|} 
LongRank \\
Test 12.4 \\
$(0 \text { to } 0)^{3}$
\end{tabular} & - & $\begin{array}{l}\text { LOव } \\
\text { LOW }\end{array}$ & CRITICAL \\
\hline \multicolumn{13}{|c|}{ Peak IOP reduction (IOPR) from baseline to (better indicated by lower values) } \\
\hline 8 & \begin{tabular}{|l|} 
randomised \\
trials
\end{tabular} & serious ${ }^{4}$ & serious $^{5}$ & serious $^{6}$ & $\begin{array}{l}\text { no serious } \\
\text { imprecision }\end{array}$ & none & 1.174 & 1.281 & - & \begin{tabular}{|c|} 
MD 0.24 \\
higher \\
$(0.57$ \\
lower to \\
1.04 \\
higher $)^{7}$
\end{tabular} & \begin{tabular}{|l|} 
पिएव \\
VERY \\
LOW
\end{tabular} & CRITICAL \\
\hline \multicolumn{13}{|c|}{ Adverse effects: burning and stinging } \\
\hline 5 & \begin{tabular}{|l} 
randomised \\
trials
\end{tabular} & $\begin{array}{l}\text { no } \\
\text { serious } \\
\text { risk of } \\
\text { bias }\end{array}$ & serious $^{8}$ & \begin{tabular}{|l|} 
no serious \\
indirectness
\end{tabular} & serious ${ }^{9}$ & none & $\begin{array}{c}134 / 1.175 \\
(11.4 \%)\end{array}$ & \begin{tabular}{|c|}
$100 / 1.281$ \\
$(7.8 \%)$
\end{tabular} & \begin{tabular}{|c|} 
RR 1.14 \\
$(0.61$ to \\
$2.14)$
\end{tabular} & $\begin{array}{c}11 \text { more } \\
\text { per } \\
1.000 \\
\text { (from } 30 \\
\text { fewer to } \\
89 \\
\text { more) }\end{array}$ & \begin{tabular}{|l|} 
LOप \\
LOW
\end{tabular} & CRITICAL \\
\hline & cts & ergy & & & & & & & & & & \\
\hline
\end{tabular}




\begin{tabular}{|l|l|l|l|l|l|c|c|c|c|c|c|}
\hline 5 & $\begin{array}{l}\text { randomised } \\
\text { trials } \\
\text { serious } \\
\text { risk of } \\
\text { bias }\end{array}$ \\
serious
\end{tabular}

${ }^{1}$ Kuprin_2011.

2 Total population size is less than 400 .

${ }^{3} p=0,01$. The log-rank test was used to compare the time to field progression between treatment groups.

${ }^{4}$ One of the studies did not specify methods of randomization, allocation concealment or blinding.

${ }^{5} \mathrm{I}$-squared value of $91 \%(\mathrm{p}<0.00001)$ indicates high statistical heterogeneity.

6 OAG progression as inferred from IOP.

${ }^{7}$ Mesuared by $\mathrm{mm} \mathrm{Hg}$.

${ }^{8} \mathrm{I}$-squared value of $71,1 \%(p<0.008)$ indicates high statistical heterogeneity.

${ }^{9}$ Low number of events. $95 \% \mathrm{Cl}$ does not exclude appreciable benefit or harm.

${ }^{10}$ This outcome does not directly measure the adverses effects. 
4.2.1.6. Análogos de las prostaglandinas

Question: Should comparisons of travoprost and latanoprost be used for primary open angle glaucoma and ocular hypertension?

Bibliography:

Eyawo O, Nachega J, Lefebvre P, Meyer D, Rachlis B, Lee C, Kelly S, Mills E. Efficacy and safety of prostaglandin analogues in patients with predominantly primary open-angle glaucoma or ocular hypertension: a meta-analysis. Clinical Ophthalmology. 2009; 3: 447-56.

\begin{tabular}{|c|c|c|c|c|c|c|c|c|c|c|c|c|}
\hline \multicolumn{7}{|c|}{ Quality assessment } & \multicolumn{2}{|c|}{ No of patients } & \multicolumn{2}{|c|}{ Effect } & \multirow{2}{*}{ Quality } & \multirow{2}{*}{ Importance } \\
\hline $\begin{array}{l}\text { No of } \\
\text { studies }\end{array}$ & Design & $\begin{array}{l}\text { Risk of } \\
\text { bias }\end{array}$ & Inconsistency & Indirectness & Imprecision & \begin{tabular}{c|} 
Other \\
considerations
\end{tabular} & travoprost & Latanoprost & \begin{tabular}{|l|} 
Relative \\
$(95 \% \mathrm{Cl})$
\end{tabular} & Absolute & & \\
\hline \multicolumn{13}{|c|}{ Weighted mean difference of IOP (better indicated by lower values) } \\
\hline 9 & $\begin{array}{l}\text { randomised } \\
\text { trials }\end{array}$ & \begin{tabular}{|l} 
no \\
serious \\
risk of \\
bias
\end{tabular} & serious $^{1}$ & serious $^{2}$ & $\begin{array}{l}\text { no serious } \\
\text { imprecision }\end{array}$ & none & 602 & 496 & - & \begin{tabular}{|c|} 
MD 0.24 \\
lower \\
$(0.87$ \\
lower to \\
0.38 \\
higher $)^{3}$
\end{tabular} & \begin{tabular}{|l|} 
Lिएव \\
LOW
\end{tabular} & CRITICAL \\
\hline \multicolumn{13}{|c|}{ Response rates } \\
\hline 2 & $\begin{array}{l}\text { randomised } \\
\text { trials }\end{array}$ & serious ${ }^{4}$ & \begin{tabular}{|l|} 
no serious \\
inconsistency
\end{tabular} & serious $^{2}$ & $\begin{array}{l}\text { no serious } \\
\text { imprecision }\end{array}$ & none & \begin{tabular}{|l|}
$194 / 365$ \\
$(53.2 \%)$
\end{tabular} & $\begin{array}{l}165 / 357 \\
(46.2 \%)\end{array}$ & \begin{tabular}{|c|}
$\mathrm{RR}$ \\
1.15 \\
$(0.99$ to \\
$1.33)$
\end{tabular} & \begin{tabular}{|c|}
69 more \\
per 1.000 \\
(from 5 \\
fewer to \\
153 \\
more)
\end{tabular} & \begin{tabular}{|l|} 
Lिएव \\
LOW
\end{tabular} & CRITICAL \\
\hline \multicolumn{13}{|c|}{ Conjunctival hyperaemia } \\
\hline 6 & randomised & no & serious $^{6}$ & no serious & serious $^{7}$ & none $^{5}$ & - & - & RR & - & 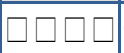 & CRITICAL \\
\hline
\end{tabular}




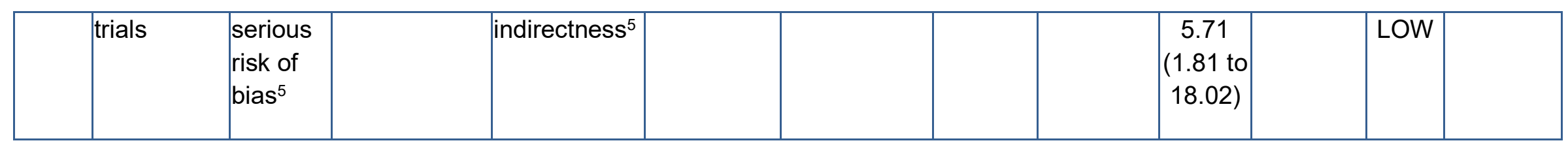

${ }^{1}$ I-squared value of $56 \%(p=0,01)$ indicates high statistical heterogeneity.

${ }^{2}$ OAG progression as inferred from IOP.

${ }^{3}$ Measured by $\mathrm{mm} \mathrm{Hg}$.

${ }^{4}$ One of the studies did not describe the allocation concelament.

${ }^{5}$ Data not available.

${ }^{6}$ I-squared value of $97 \%(p=0,001)$ indicates high statistical heterogeneity

${ }^{7}$ Wide confidence interval, $95 \% \mathrm{Cl}$ does not exclude appreciable benefit or harm.

Question: Should comparisons of travoprost and bimatoprost be used for primary open angle glaucoma and ocular hypertension?

\section{Bibliography:}

Eyawo O, Nachega J, Lefebvre P, Meyer D, Rachlis B, Lee C, Kelly S, Mills E. Efficacy and safety of prostaglandin analogues in patients with predominantly primary open-angle glaucoma or ocular hypertension: a meta-analysis. Clinical Ophthalmology. 2009; 3: 447-56.

Chander A, Kapoor C, Thomas S. Comparison of the efficacy ad safety of bimatoprost $(0.03 \%)$ and travaprost $(0.004 \%)$ in patients with primary open angle glaucoma. Nepal J Ophthalmol. 2013; 5 (9): 75-80.

\begin{tabular}{|c|c|c|c|c|c|c|c|c|c|c|c|c|}
\hline \multicolumn{7}{|c|}{ Quality assessment } & \multicolumn{2}{|c|}{ No of patients } & \multicolumn{2}{|c|}{ Effect } & \multirow{2}{*}{ Quality } & \multirow{2}{*}{ Importance } \\
\hline \begin{tabular}{|l} 
No of \\
studies
\end{tabular} & Design & \begin{tabular}{|c|}
$\begin{array}{c}\text { Risk of } \\
\text { bias }\end{array}$ \\
\end{tabular} & Inconsistency & Indirectness & Imprecision & \begin{tabular}{|c|}
$\begin{array}{c}\text { Other } \\
\text { considerations }\end{array}$ \\
\end{tabular} & travoprost & Bimatoprost & \begin{tabular}{|l} 
Relative \\
$(95 \% \mathrm{Cl})$
\end{tabular} & Absolute & & \\
\hline \multicolumn{13}{|c|}{ Weighted mean difference of IOP (better indicated by lower values) } \\
\hline 8 & $\begin{array}{l}\text { randomised } \\
\text { trials }\end{array}$ & serious $^{1}$ s & serious $^{2}$ & serious $^{3}$ & $\begin{array}{l}\text { no serious } \\
\text { imprecision }\end{array}$ & none & 351 & 348 & - & $\begin{array}{l}\text { MD } 0.88 \\
\text { higher } \\
\text { ( } 0.13 \text { to }\end{array}$ & \begin{tabular}{|l|l|} 
VIDG \\
VERY \\
LOW
\end{tabular} & CRITICAL \\
\hline
\end{tabular}




\begin{tabular}{|c|c|c|c|c|c|c|c|c|c|c|c|c|}
\hline & & & & & & & & & & \begin{tabular}{c|}
1.63 \\
higher $)^{4}$
\end{tabular} & & \\
\hline \multicolumn{13}{|c|}{ Response rates } \\
\hline 3 & $\begin{array}{l}\text { randomised } \\
\text { trials }\end{array}$ & serious 1 & $\begin{array}{l}\text { no serious } \\
\text { inconsistency }\end{array}$ & serious $^{3}$ & serious ${ }^{5}$ & none & $\begin{array}{c}91 / 141 \\
(64.5 \%)\end{array}$ & $\begin{array}{c}110 / 141 \\
(78 \%)\end{array}$ & \begin{tabular}{|c|}
$\mathrm{RR}$ \\
0.82 \\
$(0.71$ to \\
$0.95)$
\end{tabular} & $\begin{array}{c}140 \\
\text { fewer per } \\
1.000 \\
\text { (from } 39 \\
\text { fewer to } \\
226 \\
\text { fewer) }\end{array}$ & \begin{tabular}{|l|} 
पिएव \\
VERY \\
LOW
\end{tabular} & CRITICAL \\
\hline \multicolumn{13}{|c|}{ Conjunctival hyperaemia } \\
\hline 1 & $\begin{array}{l}\text { randomised } \\
\text { trials }\end{array}$ & serious 6 & serious ${ }^{6,7}$ & $\begin{array}{l}\text { no serious } \\
\text { indirectness }\end{array}$ & $\begin{array}{l}\text { no serious } \\
\text { imprecision }^{6},\end{array}$ & none ${ }^{6}$ & - & - & \begin{tabular}{|c|} 
RR \\
0.82 \\
$(0.69$ to \\
$0.97)$
\end{tabular} & 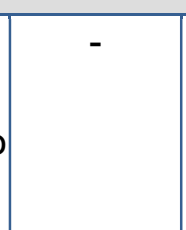 & \begin{tabular}{|l|} 
पिएव \\
LOW
\end{tabular} & CRITICAL \\
\hline \multicolumn{13}{|c|}{24 hour mean IOP reduction (\%) (12 weeks treatment) (better indicated by lower values) } \\
\hline 1 & \begin{tabular}{|l} 
randomised \\
trials
\end{tabular} & serious $^{9}$ & $\begin{array}{l}\text { no serious } \\
\text { inconsistency }\end{array}$ & serious $^{3}$ & $\begin{array}{l}\text { very } \\
\text { serious }{ }^{10}\end{array}$ & none & $\begin{array}{c}4 / 15 \\
(28 \%)\end{array}$ & $\begin{array}{c}6 / 16 \\
(35 \%)\end{array}$ & - & $\begin{array}{c}\text { not } \\
\text { pooled }\end{array}$ & $\begin{array}{l}\text { GQTि } \\
\text { VERY } \\
\text { LOW }\end{array}$ & CRITICAL \\
\hline
\end{tabular}

${ }^{1}$ Some of studies did not specify methods of randomization, allocation concealment or blinding. 2 I-squared value of $56 \%$ indicates high statistical heterogeneity.

${ }^{3}$ OAG progression as inferred from IOP.

${ }^{4}$ Measured by $\mathrm{mm} \mathrm{Hg}$.

${ }^{5}$ Low number of events. $95 \% \mathrm{Cl}$ does not exclude appreciable benefit or harm.

${ }^{6}$ Data not available.

7 Heterogeneity is suspected, $p=0.02$

8 Not suspected due to the narrow IC $95 \%$

9 Did not specify methods of allocation concealment or blinding.

Did not specify methods of, allocation concealment or blinding. 
Question: Should comparison of latanoprost frente bimatoprost be used for primary open angle glaucoma and ocular hypertension? Bibliography:

Eyawo O, Nachega J, Lefebvre P, Meyer D, Rachlis B, Lee C, Kelly S, Mills E. Efficacy and safety of prostaglandin analogues in patients with predominantly primary open-angle glaucoma or ocular hypertension: a meta-analysis. Clinical Ophthalmology. 2009; 3: 447-56.

\begin{tabular}{|c|c|c|c|c|c|c|c|c|c|c|c|c|}
\hline \multicolumn{7}{|c|}{ Quality assessment } & \multicolumn{2}{|c|}{ No of patients } & \multicolumn{2}{|c|}{ Effect } & \multirow{2}{*}{ Quality } & \multirow{2}{*}{ Importance } \\
\hline \begin{tabular}{|l|} 
No of \\
studies
\end{tabular} & Design & \begin{tabular}{|c}
$\begin{array}{c}\text { Risk of } \\
\text { bias }\end{array}$ \\
\end{tabular} & Inconsistency & Indirectness & Imprecision & \begin{tabular}{c|c|} 
Other \\
considerations
\end{tabular} & Iatanoprost & Bimatoprost & \begin{tabular}{|l|} 
Relative \\
$(95 \% \mathrm{Cl})$
\end{tabular} & Absolute & & \\
\hline \multicolumn{13}{|c|}{ Weighted mean difference of IOP (better indicated by lower values) } \\
\hline 8 & $\begin{array}{l}\text { randomised } \\
\text { trials }\end{array}$ & serious $^{1}$ & 1 serious $^{2}$ & serious $^{3}$ & \begin{tabular}{|l|} 
no serious \\
imprecision
\end{tabular} & none & 482 & 492 & - & \begin{tabular}{|c|} 
MD 0.73 \\
higher \\
(0.1 to \\
1.37 \\
${\text { higher })^{4}}^{4}$
\end{tabular} & $\begin{array}{l}\text { VQTि } \\
\text { VERY } \\
\text { LOW }\end{array}$ & CRITICAL \\
\hline \multicolumn{13}{|c|}{ Response rates } \\
\hline 3 & \begin{tabular}{|l} 
randomised \\
trials
\end{tabular} & serious $^{1}$ & 1 serious & serious $^{3}$ & \begin{tabular}{|l|} 
no serious \\
imprecision
\end{tabular} & none & $\begin{array}{l}178 / 275 \\
(64.7 \%)\end{array}$ & $\begin{array}{l}192 / 275 \\
(69.8 \%)\end{array}$ & \begin{tabular}{|c|}
$R R$ \\
0.98 \\
$(0.76$ to \\
$1.26)$
\end{tabular} & \begin{tabular}{|c|}
14 fewer \\
per 1.000 \\
(from 168 \\
fewer to \\
182 more)
\end{tabular} & \begin{tabular}{|l|} 
VIDG \\
VERY \\
LOW \\
\end{tabular} & CRITICAL \\
\hline \multicolumn{13}{|c|}{ Conjunctival hyperaemia } \\
\hline 5 & \begin{tabular}{|l} 
randomised \\
trials
\end{tabular} & serious $^{5}$ & serious ${ }^{2}$ & \begin{tabular}{|l|} 
no serious \\
indirectness
\end{tabular} & \begin{tabular}{|l} 
no serious \\
imprecision
\end{tabular} & none $^{5}$ & - & - & \begin{tabular}{|c|}
$\mathrm{RR}$ \\
1.59 \\
$(1.02$ to \\
$2.48)$
\end{tabular} & 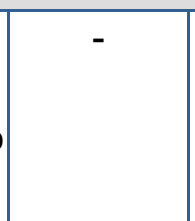 & $\begin{array}{l}\text { पिवर् } \\
\text { LOW }\end{array}$ & CRITICAL \\
\hline
\end{tabular}


${ }^{1}$ Some of studies did not specify methods of randomization, allocation concealment or blinding.

${ }^{2}$ I-squared value of $47 \%(p=0,06)$ indicates high statistical heterogeneity.

3 OAG progression as inferred from IOP

Measured by $\mathrm{mm} \mathrm{Hg}$

${ }^{5}$ Data not available

4.2.1.7. Análogos de prostaglandinas frente a agonista alfa selectivos

Question: Should comparison of latanoprost and brimonidine be used for primary open angle glaucoma and ocular hypertension? Bibliography:

Hodge WG, Lachaine J, Steffensen I, Murray C, Barnes D, Foerster V, Ducruet T, Morrison A. The efficacy and harm of prostaglandin analogues for IOP reduction in glaucoma patients compared to dorzolamide and brimonidine: a systematic review. Br J Ophthalmol. 2008; 92(1): 7-12.

\begin{tabular}{|c|c|c|c|c|c|c|c|c|c|c|c|c|}
\hline \multicolumn{7}{|c|}{ Quality assessment } & \multicolumn{2}{|c|}{ No of patients } & \multicolumn{2}{|c|}{ Effect } & \multirow{2}{*}{ Quality } & \multirow{2}{*}{ Importance } \\
\hline \begin{tabular}{|l}
$\begin{array}{l}\text { No of } \\
\text { studies }\end{array}$ \\
sudis
\end{tabular} & Design & $\begin{array}{c}\text { Risk of } \\
\text { bias }\end{array}$ & Inconsistency & Indirectness & Imprecision & $\begin{array}{c}\text { Other } \\
\text { considerations }\end{array}$ & Latanoprost & Brimonidine & \begin{tabular}{|l|} 
Relative \\
$(95 \% \mathrm{Cl})$
\end{tabular} \mid & Absolute & & \\
\hline \multicolumn{13}{|c|}{ Weighted mean difference of IOP (better indicated by lower values) } \\
\hline 3 & $\begin{array}{l}\text { randomisec } \\
\text { trials }\end{array}$ & serious $\mathbf{s}^{1}$ & serious ${ }^{2}$ & serious $^{3}$ & \begin{tabular}{|l|} 
no serious \\
imprecision
\end{tabular} & none & 236 & 235 & - & \begin{tabular}{|c} 
MD 1.04 \\
lower \\
$(3.01$ \\
lower to \\
0.93 \\
higher $)^{4}$
\end{tabular} & \begin{tabular}{|l|}
$\square \square \square \square$ \\
VERY \\
LOW
\end{tabular} & CRITICAL \\
\hline \multicolumn{13}{|c|}{ Withdrawals due to adverse events } \\
\hline 4 & $\begin{array}{l}\text { randomise } \\
\text { trials }\end{array}$ & serious ${ }^{1}$ & $\begin{array}{l}\text { no serious } \\
\text { inconsistency5 }\end{array}$ & serious $^{6}$ & serious $^{7}$ & none & $\begin{array}{l}12 / 423 \\
(2.8 \%)\end{array}$ & $\begin{array}{l}46 / 423 \\
(10.9 \%)\end{array}$ & \begin{tabular}{|c|}
$R R$ \\
0.51 \\
$(0.09$ to
\end{tabular} & $\begin{array}{l}53 \text { fewer } \\
\text { per } 1.000 \\
\text { (from } 99\end{array}$ & $\begin{array}{l}\text { Vदिए } \\
\text { VERY }\end{array}$ & CRITICAL \\
\hline
\end{tabular}




\begin{tabular}{|c|c|c|c|c|c|c|c|c|c|c|c|c|}
\hline & & & & & & & & & 2) & \begin{tabular}{|c|} 
fewer to \\
109 \\
more $)$
\end{tabular} & LOW & \\
\hline \multicolumn{13}{|c|}{ Ocular hyperaemia } \\
\hline 2 & $\begin{array}{l}\text { randomised } \\
\text { trials }\end{array}$ & serious $^{8}$ & 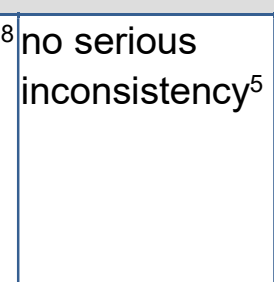 & $\begin{array}{l}\text { no serious } \\
\text { indirectness }\end{array}$ & serious $^{7}$ & none & $\begin{array}{l}18 / 248 \\
(7.3 \%)\end{array}$ & $\begin{array}{c}15 / 252 \\
(6 \%)\end{array}$ & $\mid \begin{array}{c}\mathrm{RR} \\
1.22 \\
(0.63 \text { to } \\
2.37)\end{array}$ & $\begin{array}{c}13 \text { more } \\
\text { per } 1.000 \\
\text { (from } 22 \\
\text { fewer to } \\
82 \text { more) }\end{array}$ & $\begin{array}{l}\text { प्र०ण } \\
\text { LOW }\end{array}$ & CRITICAL \\
\hline \multicolumn{13}{|c|}{ All other ocular adverse events (excluding hyperemia) } \\
\hline 3 & $\begin{array}{l}\text { randomised } \\
\text { trials }\end{array}$ & serious 1 & $\begin{array}{l}1 \text { no serious } \\
\text { inconsistency }\end{array}$ & \begin{tabular}{|l|} 
no serious \\
indirectness
\end{tabular} & serious $^{7}$ & none & $\begin{array}{l}82 / 400 \\
(20.5 \%)\end{array}$ & $\begin{array}{c}125 / 403 \\
(31 \%)\end{array}$ & \begin{tabular}{|c|}
$\mathrm{RR}$ \\
0.66 \\
$(0.52$ to \\
$0.83)$ \\
\\
\end{tabular} & $\mid \begin{array}{c}105 \\
\text { fewer per } \\
1.000 \\
\text { (from } 53 \\
\text { fewer to } \\
149 \\
\text { fewer) }\end{array}$ & $\begin{array}{l}\text { Lup } \\
\text { LOW }\end{array}$ & CRITICAL \\
\hline \multicolumn{13}{|c|}{ Serious adverses events } \\
\hline 3 & $\begin{array}{l}\text { randomisec } \\
\text { trials }\end{array}$ & serious $^{8}$ & $\begin{array}{l}8 \text { no serious } \\
\text { inconsistency }\end{array}$ & \begin{tabular}{|l|} 
no serious \\
indirectness
\end{tabular} & serious $^{7}$ & none & $\begin{array}{l}8 / 400 \\
(2 \%)\end{array}$ & $\begin{array}{l}13 / 403 \\
(3.2 \%)\end{array}$ & \begin{tabular}{|c|}
$R R$ \\
0.60 \\
$(0.25$ to \\
$1.45)$
\end{tabular} & $\begin{array}{l}13 \text { fewer } \\
\text { per } 1.000 \\
\text { (from } 24 \\
\text { fewer to } \\
15 \text { more) }\end{array}$ & $\begin{array}{l}\text { पिपा } \\
\text { LOW }\end{array}$ & CRITICAL \\
\hline
\end{tabular}

1 Jadad score $=2$, one of the studies did not specify methods of randomization, allocation concealment or blinding 2 I-squared value of $87.5 \%(p<0.0003)$ indicates high statistical heterogeneity.

3 OAG progression as inferred from IOP.

${ }^{4}$ Measured by $\mathrm{mm} \mathrm{Hg}$.

5 Data no provided.

This outcome does not directly measure the adverse effects. 
${ }^{7}$ Low number of events. $95 \% \mathrm{Cl}$ does not exclude appreciable benefit or harm.

7

\subsubsection{Betabloqueadores}

Question: Comparison of timolol and carteolol for primary open angle glaucoma and ocular hypertension [Data only. When citing this record quote "Cochrane Database of Systematic Reviews 2007, Issue 4".]

Bibliography:

Vass C, Hirn C, Sycha T, Findl O, Sacu S, Bauer P. Schmetterer L. Medical interventions for primary open angle glaucoma and ocular hypertension [Data only. When citing this record quote "Cochrane Database of Systematic Reviews 2007, Issue 4".]. Cochrane Database of Systematic Reviews [Year], Issue [Issue].

\begin{tabular}{|c|c|c|c|c|c|c|c|c|c|c|c|c|}
\hline \multicolumn{7}{|c|}{ Quality assessment } & \multicolumn{2}{|c|}{ No of patients } & \multicolumn{2}{|c|}{ Effect } & \multirow{2}{*}{ Quality } & \multirow{2}{*}{ Importance } \\
\hline $\begin{array}{l}\text { No of } \\
\text { studies }\end{array}$ & Design & $\begin{array}{c}\text { Risk } \\
\text { of bias }\end{array}$ & Inconsistenc) & |lndirectness & Imprecision & $\begin{array}{c}\text { Other } \\
\text { considerations }\end{array}$ & timolol & Carteolol & $\begin{array}{c}\text { Relative } \\
(95 \% \\
\text { Cl) }\end{array}$ & Absolute & & \\
\hline \multicolumn{13}{|c|}{ Incidence of visual field defect progression } \\
\hline 2 & $\begin{array}{l}\text { randomisec } \\
\text { trials }\end{array}$ & $\begin{array}{l}\text { no } \\
\text { serious } \\
\text { risk of } \\
\text { bias }\end{array}$ & $\begin{array}{l}\text { no serious } \\
\text { inconsistency }\end{array}$ & $\begin{array}{l}\text { no serious } \\
\text { indirectness }\end{array}$ & serious $^{1}$ & none & $\begin{array}{c}1 / 88 \\
(1.1 \%)\end{array}$ & $\begin{array}{c}9 / 83 \\
(10.8 \%)\end{array}$ & $\begin{array}{c}\text { OR } 0.18 \\
(0.05 \text { to } \\
0.62)\end{array}$ & \begin{tabular}{|c|}
87 fewer \\
per 1.000 \\
(from 38 \\
fewer to \\
102 \\
fewer)
\end{tabular} & $\begin{array}{c}\text { MUपि } \\
\text { MODERATE }\end{array}$ & CRITICAL \\
\hline
\end{tabular}

${ }^{1}$ Low number of events. 95\% Cl does not exclude appreciable benefit or harm. 
Question: Comparison of timolol and levobunolol for primary open angle glaucoma and ocular hypertension [Data only. When citing this record quote "Cochrane Database of Systematic Reviews 2007, Issue 4".]

\section{Bibliography:}

Vass C, Hirn C, Sycha T, Findl O, Sacu S, Bauer P, Schmetterer L. Medical interventions for primary open angle glaucoma and ocula hypertension [Data only. When citing this record quote "Cochrane Database of Systematic Reviews 2007, Issue 4".]. Cochrane Database of Systematic Reviews [Year], Issue [Issue].

\begin{tabular}{|c|c|c|c|c|c|c|c|c|c|c|c|c|}
\hline \multicolumn{7}{|c|}{ Quality assessment } & \multicolumn{2}{|c|}{ No of patients } & \multicolumn{2}{|c|}{ Effect } & \multirow{2}{*}{ Quality } & \multirow{2}{*}{ Importance } \\
\hline $\begin{array}{l}\text { No of } \\
\text { studies }\end{array}$ & Design & $\begin{array}{c}\text { Risk of } \\
\text { bias }\end{array}$ & Inconsistency & Indirectness & Imprecision & $\begin{array}{c}\text { Other } \\
\text { considerations }\end{array}$ & timolol & Levobunolol & $\begin{array}{l}\text { Relative } \\
(95 \% \mathrm{Cl})\end{array}$ & Absolute & & \\
\hline \multicolumn{13}{|c|}{ Incidence of visual field defect progression } \\
\hline 2 & $\begin{array}{l}\text { randomisec } \\
\text { trials }\end{array}$ & $\begin{array}{l}\text { no } \\
\text { serious } \\
\text { risk of } \\
\text { bias }\end{array}$ & $\begin{array}{l}\text { no serious } \\
\text { inconsistency }\end{array}$ & $\begin{array}{l}\text { no serious } \\
\text { indirectness }\end{array}$ & serious $^{1}$ & none & $\begin{array}{l}32 / 141 \\
(22.7 \%)\end{array}$ & $\begin{array}{c}18 / 149 \\
(12.1 \%)\end{array}$ & $\begin{array}{c}\text { OR 2.2 } \\
(1.17 \\
\text { to } \\
4.14)\end{array}$ & \begin{tabular}{|c|}
111 \\
more per \\
1.000 \\
(from 18 \\
more to \\
242 \\
more)
\end{tabular} & MODERATE & CRITICAL \\
\hline \multicolumn{13}{|c|}{ Drop-out due to drug-related adverse events } \\
\hline 2 & $\begin{array}{l}\text { randomisec } \\
\text { trials }\end{array}$ & $\begin{array}{l}\text { no } \\
\text { serious } \\
\text { risk of } \\
\text { bias }\end{array}$ & serious $^{2}$ & serious $^{3}$ & serious $^{1}$ & none & $\begin{array}{l}10 / 141 \\
(7.1 \%)\end{array}$ & $\begin{array}{l}13 / 149 \\
(8.7 \%)\end{array}$ & $\begin{array}{c}\text { OR } 0.8 \\
(0.34 \\
\text { to } \\
1.87)\end{array}$ & $\begin{array}{c}16 \text { fewer } \\
\text { per } \\
1.000 \\
\text { (from } 56 \\
\text { fewer to } \\
64 \text { more) }\end{array}$ & $\begin{array}{c}\text { पिपा } \\
\text { VERY LOW }\end{array}$ & CRITICAL \\
\hline
\end{tabular}


1 Low number of events. $95 \% \mathrm{Cl}$ does not exclude appreciable benefit or harm

${ }^{2}$ I-squared value of $66 \%(p=0,09)$ indicates high statistical heterogeneity.

${ }^{3}$ This outcome does not directly measure the adverses effects.

Question: Comparisons of timolol and betaxolol for primary open angle glaucoma and ocular hypertension [Data only. When citing this record quote "Cochrane Database of Systematic Reviews 2007, Issue 4".]

Bibliography:

Vass C, Hirn C, Sycha T, Findl O, Sacu S, Bauer P, Schmetterer L. Medical interventions for primary open angle glaucoma and ocular hypertension [Data only. When citing this record quote "Cochrane Database of Systematic Reviews 2007, Issue 4".]. Cochrane Database of Systematic Reviews [Year], Issue [Issue].

\begin{tabular}{|c|c|c|c|c|c|c|c|c|c|c|c|c|}
\hline \multicolumn{7}{|c|}{ Quality assessment } & \multicolumn{2}{|c|}{ No of patients } & \multicolumn{2}{|c|}{ Effect } & \multirow{2}{*}{ Quality } & \multirow{2}{*}{ Importance } \\
\hline \begin{tabular}{|c|} 
No of \\
studies
\end{tabular} & Design & $\begin{array}{l}\text { Risk of } \\
\text { bias }\end{array}$ & Inconsistency & Indirectness & Imprecision & \begin{tabular}{|c} 
Other \\
considerations
\end{tabular} & timolol & betaxolol & \begin{tabular}{|l|} 
Relative \\
$(95 \% \mathrm{Cl})$
\end{tabular} & Absolute & & \\
\hline \multicolumn{13}{|c|}{ Change of visual field mean sensitivity (Better indicated by lower values) } \\
\hline 6 & $\begin{array}{l}\text { randomisec } \\
\text { trials }\end{array}$ & serious $^{1}$ & serious $^{2}$ & $\begin{array}{l}\text { no serious } \\
\text { indirectness }\end{array}$ & serious $^{3}$ & none & 127 & 131 & - & $\begin{array}{c}\text { MD } 0.07 \\
\text { higher }(0.43 \\
\text { lower to } \\
0.57 \\
\text { higher })^{4}\end{array}$ & \begin{tabular}{|l|l|} 
पि口प \\
VERY \\
LOW
\end{tabular} & CRITICAL \\
\hline \multicolumn{13}{|c|}{ Drop-out due to drug-related adverse events } \\
\hline 5 & $\begin{array}{l}\text { randomisec } \\
\text { trials }\end{array}$ & serious $^{1}$ & $\begin{array}{l}\text { no serious } \\
\text { inconsistency }\end{array}$ & serious $^{5}$ & serious ${ }^{6}$ & none & $\begin{array}{l}17 / 117 \\
(14.5 \%)\end{array}$ & $\begin{array}{l}8 / 121 \\
(6.6 \%)\end{array}$ & \begin{tabular}{|c|} 
OR 2.4 \\
(1.04 to \\
$5.53)$
\end{tabular} & \begin{tabular}{|c}
79 more per \\
1.000 (from \\
2 more to \\
215 more)
\end{tabular} & $\begin{array}{l}\text { G०पि } \\
\text { VERY } \\
\text { LOW }\end{array}$ & CRITICAL \\
\hline
\end{tabular}


${ }^{1}$ Some of studies did not specify methods of randomization, allocation concealment or blinding.

${ }^{2}$ I-squared value of $85 \%(p=0,001)$ indicates high statistical heterogeneity.

Total population size is less than 400 .

${ }^{4}$ Measured in $\mathrm{Db}$.

5 This outcome does not directly measure the adverses effects.

${ }^{6}$ Low number of events. $95 \% \mathrm{Cl}$ does not exclude appreciable benefit or harm.

\subsubsection{Uso de combinaciones fijas de fármacos}

Question: Should Fixed combinations vs unfixed combinations be used for primary open angle glaucoma and ocular hypertension?

\section{Bibliography:}

Quaranta L, Biagioli E, Riva I, Rulli E, Poli D, Katsanos A, Floriani I. Prostaglandin analogs and timolol-fixed versus unfixed combinations or monotherapy for open-angle glaucoma: a systematic review and meta-analysis. J Ocul Pharmacol Ther. 2013 May; 29(4): 382-9.

\begin{tabular}{|c|c|c|c|c|c|c|c|c|c|c|c|c|}
\hline \multicolumn{7}{|c|}{ Quality assessment } & \multicolumn{2}{|c|}{ No of patients } & \multicolumn{2}{|c|}{ Effect } & \multirow{2}{*}{ Quality } & \multirow{2}{*}{ Importance } \\
\hline $\begin{array}{l}\begin{array}{l}\text { No of } \\
\text { studies }\end{array} \\
\end{array}$ & Design & $\begin{array}{c}\text { Risk of } \\
\text { bias }\end{array}$ & Inconsistency & Indirectness & Imprecision & $\begin{array}{c}\text { Other } \\
\text { considerations }\end{array}$ & $\begin{array}{c}\text { Fixed } \\
\text { combinations }\end{array}$ & \begin{tabular}{c|c} 
Unfixed \\
combinations
\end{tabular} & \begin{tabular}{|l|} 
Relative \\
$(95 \%$ Cl)
\end{tabular} & Absolute & & \\
\hline \multicolumn{13}{|c|}{ Control IOP (Better indicated by lower values) } \\
\hline 5 & $\begin{array}{l}\text { randomisec } \\
\text { trials }\end{array}$ & serious ${ }^{1}$ & serious $^{2}$ & serious $^{3}$ & $\begin{array}{l}\text { no serious } \\
\text { imprecision }\end{array}$ & none & & & & 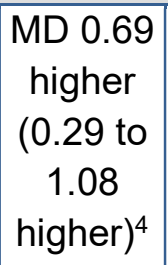 & \begin{tabular}{|l|} 
पिएव \\
VERY \\
LOW
\end{tabular} & CRITICAL \\
\hline \multicolumn{13}{|c|}{ Adverse effects: conjunctival hyperemia } \\
\hline 4 & $\begin{array}{l}\text { randomisec } \\
\text { trials }\end{array}$ & serious ${ }^{1}$ & serious $^{2}$ & $\begin{array}{l}\text { no serious } \\
\text { indirectness }\end{array}$ & serious $^{5}$ & none & $\begin{array}{l}60 / 709 \\
(8.5 \%)\end{array}$ & $\begin{array}{c}86 / 691 \\
(12.4 \%)\end{array}$ & $\begin{array}{l}\text { RR } \\
0.70\end{array}$ & $\begin{array}{c}37 \text { fewer } \\
\text { per }\end{array}$ & \begin{tabular}{|l|} 
पिमा \\
VERY
\end{tabular} & CRITICAL \\
\hline
\end{tabular}




\begin{tabular}{|l|l|l|l|l|l|l|l|c|c|c|}
\hline & & & & & & & & $\left(\begin{array}{c}0.43 \text { to } \\
1.000 \\
1.14) \\
\text { (from } 71 \\
\text { fewer to } \\
17 \text { more })\end{array}\right.$ & LOW \\
& & & & & & & & \\
\hline
\end{tabular}

Some of studies did not specify methods of randomization, allocation concealment, blinding or dosing schemes. 2 I-squared value of $52 \%(p=0,08)$ indicates statistical heterogeneity.

OAG progression as inferred from IOP.

${ }^{4}$ Measured in $\mathrm{mmHg}$.

${ }^{5}$ Low number of events. $95 \% \mathrm{Cl}$ does not exclude appreciable benefit or harm. 


\subsubsection{Uso de fármacos libres de preservantes}

Question: Should preservative-free pharmacological treatment vs preservative pharmacological treatment be used for glaucoma and ocular hypertension?

Bibliography:

Lewis RA, Katz GJ, Weiss MJ, Landry TA, Dickerson JE, James JE, Hua SY, Sullivan EK, Montgomery DB, Wells DT, Bergamini MV; Travoprost BAC-free Study Group. Travoprost $0.004 \%$ with and without benzalkonium chloride: a comparison of safety and efficacy. $J$ Glaucoma. 2007; 16(1): 98-103.

Shedden A, Adamsons IA, Getson AJ, Laurence JK, Lines CR, Hewitt DJ, Ho TW. Comparison of the efficacy and tolerability of preservative-free and preservative-containing formulations of the dorzolamide/timolol fixed combination (COSOPTâ, $\phi$ ) in patients with elevated intraocular pressure in a randomized clinical trial. Graefes Arch Clin Exp Ophthalmol. 2010; 248(12): 1757-64.

Day DG, Walters TR, Schwartz GF, Mundorf TK, Liu C, Schiffman RM, Bejanian M. Bimatoprost $0.03 \%$ preservative-free ophthalmic solution versus bimatoprost $0.03 \%$ ophthalmic solution (Lumigan) for glaucoma or ocular hypertension: a 12-week, randomised, doublemasked trial. Br J Ophthalmol. 2013; 97(8): 989-93.

Rouland JF, Traverso CE, Stalmans I, Fekih LE, Delval L, Renault D, Baudouin C; T2345 Study Group. Efficacy and safety of preservativefree latanoprost eyedrops, compared with BAK-preserved latanoprost in patients with ocular hypertension or glaucoma. $\mathrm{Br} \mathrm{J}$ Ophthalmol. 2013; 97(2): 196-200

\begin{tabular}{|c|c|c|c|c|c|c|c|c|c|c|c|c|}
\hline \multicolumn{7}{|c|}{ Quality assessment } & \multicolumn{2}{|c|}{ No of patients } & \multicolumn{2}{|c|}{ Effect } & \multirow{2}{*}{ Quality } & \multirow{2}{*}{ Importance } \\
\hline \begin{tabular}{|c|} 
No of \\
studies
\end{tabular} & Design & $\begin{array}{l}\text { Risk of } \\
\text { bias }\end{array}$ & Inconsistency & Indirectness & Imprecision & $\begin{array}{c}\text { Other } \\
\text { considerations }\end{array}$ & $\begin{array}{c}\text { Preservative- } \\
\text { free } \\
\text { pharmacological } \\
\text { treatment }\end{array}$ & $\begin{array}{c}\text { Preservative } \\
\text { pharmacological } \\
\text { treatment }\end{array}$ & $\begin{array}{c}\text { Relative } \\
(95 \% \\
\text { CI) }\end{array}$ & Absolute & & \\
\hline \multicolumn{13}{|c|}{ Adverse effects: conjunctival hyperemia } \\
\hline 3 & $\begin{array}{l}\text { randomise } \\
\text { trials }\end{array}$ & serious $^{1}$ & \begin{tabular}{|l|} 
no serious \\
inconsistency
\end{tabular} & $\begin{array}{l}\text { no serious } \\
\text { indirectness }\end{array}$ & $\begin{array}{l}\text { no serious } \\
\text { imprecision }\end{array}$ & none & $\begin{array}{c}137 / 858 \\
(16 \%)\end{array}$ & $\begin{array}{l}162 / 830 \\
(19.5 \%)\end{array}$ & $\begin{array}{c}\text { RR } \\
0.81 \\
(0.66 \\
\text { to }\end{array}$ & \begin{tabular}{|c|}
37 \\
fewer \\
per \\
1.000
\end{tabular} & 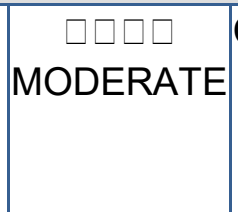 & CRITICAL \\
\hline
\end{tabular}




\begin{tabular}{|c|c|c|c|c|c|c|c|c|c|c|c|}
\hline & & & & & & & & 0.99) & $\begin{array}{c}\text { (from } 2 \\
\text { fewer } \\
\text { to } 66 \\
\text { fewer) }\end{array}$ & & \\
\hline \multicolumn{12}{|c|}{ Adverse effects: subjective ocular symptoms } \\
\hline 3 & \begin{tabular}{|l|} 
randomised \\
trials
\end{tabular} & 1 serious $^{2}$ & $\begin{array}{l}\text { no serious } \\
\text { indirectness }\end{array}$ & serious $^{3}$ & none & $\begin{array}{l}61 / 645 \\
(9.5 \%)\end{array}$ & $\begin{array}{l}87 / 614 \\
(14.2 \%)\end{array}$ & \begin{tabular}{|c|}
$\mathrm{RR}$ \\
0.67 \\
$(0.39$ \\
to \\
$1.15)$
\end{tabular} & $\begin{array}{c}47 \\
\text { fewer } \\
\text { per } \\
1.000 \\
\text { (from } \\
86 \\
\text { fewer } \\
\text { to } 21 \\
\text { more) }\end{array}$ & $\begin{array}{c}\text { पिमा } \\
\text { VERY LOW }\end{array}$ & CRITICAL \\
\hline \multicolumn{12}{|c|}{ Drop-out due to drug-related } \\
\hline 4 & \begin{tabular}{|l|} 
randomised \\
trials
\end{tabular} & $\begin{array}{l}1 \text { no serious } \\
\text { inconsistency }\end{array}$ & serious ${ }^{4}$ & serious $^{3}$ & none & $\begin{array}{l}14 / 989 \\
(1.4 \%)\end{array}$ & $\begin{array}{l}11 / 960 \\
(1.1 \%)\end{array}$ & \begin{tabular}{|c|}
$\mathrm{RR}$ \\
1.23 \\
$(0.55$ \\
to \\
$2.74)$ \\
\end{tabular} & $\begin{array}{c}3 \text { more } \\
\text { per } \\
1.000 \\
\text { (from } 5 \\
\text { fewer } \\
\text { to } 20 \\
\text { more) }\end{array}$ & \begin{tabular}{|c|} 
घप्य \\
VERY LOW
\end{tabular} & CRITICAL \\
\hline \multicolumn{12}{|c|}{ Adverse effects: Punctate keratitis } \\
\hline 2 & $\begin{array}{l}\text { randomised serious }{ }^{1} \\
\text { trials }\end{array}$ & $\begin{array}{l}1{ }^{1} \text { no serious } \\
\text { inconsistency }\end{array}$ & \begin{tabular}{|l|} 
no serious \\
yndirectness
\end{tabular} & serious $^{3}$ & none & $\begin{array}{l}31 / 432 \\
(7.2 \%)\end{array}$ & $\begin{array}{l}40 / 425 \\
(9.4 \%)\end{array}$ & \begin{tabular}{|c|}
$\mathrm{RR}$ \\
0.77 \\
$(0.5$ to \\
$1.18)$
\end{tabular} & $\begin{array}{c}22 \\
\text { fewer } \\
\text { per } \\
1.000 \\
\text { (from } \\
47\end{array}$ & $\begin{array}{l}\text { पिपा } \\
\text { LOW }\end{array}$ & CRITICAL \\
\hline
\end{tabular}




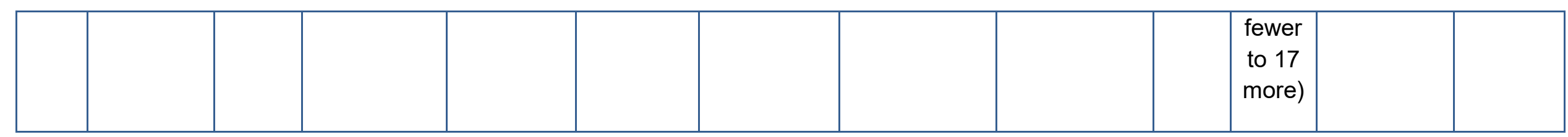

Some of studies did not specify methods of randomization, allocation concealment or blinding

${ }^{2}$ I-squared value of $66 \%(p=0,05)$ indicates statistical heterogeneity.

${ }^{3}$ Low number of events. $95 \% \mathrm{Cl}$ does not exclude appreciable benefit or harm.

${ }^{4}$ This outcome does not directly measure the adverses effects. 


\section{MÉTODOS}

Como medida del efecto, para las variables dicotómicas se utilizó el cociente de riesgos (CR) con intervalo de confianza del 95\%. Como método estadístico se utilizaron, respectivamente, los métodos de Mantel-Haenszel y de la varianza inversa. Para el metanálisis se utilizó el modelo de efectos fijos, excepto cuando se encontró heterogeneidad significativa; en ese caso, se utilizó el modelo de efectos aleatorios.

FOREST PLOTS

\section{Hiperemia conjuntival}

\begin{tabular}{|c|c|c|c|c|c|c|c|c|}
\hline \multirow[b]{2}{*}{ Study or Subgroup } & \multicolumn{2}{|c|}{ Fármacos sin conservantes } & \multicolumn{2}{|c|}{ Fármacos con conservantes } & \multirow[b]{2}{*}{ Weight } & \multirow{2}{*}{$\begin{array}{l}\text { Risk Ratio } \\
\text { M-H, Fixed, } 95 \% \mathrm{Cl} \\
\end{array}$} & \multirow{2}{*}{\multicolumn{2}{|c|}{$\begin{array}{c}\text { Risk Ratio } \\
\text { M-H, Fixed, 95\% Cl } \\
\end{array}$}} \\
\hline & Events & Total & Events & Tota & & & & \\
\hline Day 2013 & 72 & 301 & 77 & 295 & $46.9 \%$ & $0.92[0.69,1.21]$ & 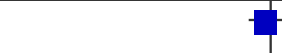 & \\
\hline Lewis 2007 & 21 & 344 & 31 & 346 & $18.6 \%$ & $0.68[0.40,1.16]$ & $\rightarrow+$ & \\
\hline Rouland 2013 & 44 & 213 & 54 & 189 & $34.5 \%$ & $0.72[0.51,1.02]$ & - & \\
\hline Total $(95 \% \mathrm{Cl})$ & & 858 & & 830 & $100.0 \%$ & $0.81[0.66,0.99]$ & $\checkmark$ & \\
\hline Total events & 137 & & 162 & & & & & \\
\hline $\begin{array}{l}\text { Heterogeneity: Chi² } \\
\text { Test for overall effect }\end{array}$ & $\begin{array}{l}1.58, \mathrm{df}=2(\mathrm{P}=0 . \\
Z=2.10(\mathrm{P}=0.04)\end{array}$ & & & & & & \begin{tabular}{cc|}
0.01 & 1 \\
0.1 & 1 \\
ivours [experimental]
\end{tabular} & $\begin{array}{c}10100 \\
\text { Favours [control] }\end{array}$ \\
\hline
\end{tabular}

\section{Síntomas objetivos oculares}

\begin{tabular}{|c|c|c|c|c|c|c|c|c|}
\hline \multirow[b]{2}{*}{ Study or Subgroup } & \multicolumn{2}{|c|}{ Fármacos sin conservantes } & \multicolumn{2}{|c|}{ Fármacos con conservantes } & \multirow[b]{2}{*}{ Weight } & \multirow{2}{*}{$\begin{array}{c}\text { Risk Ratio } \\
\text { M-H, Random, } 95 \% \text { CI }\end{array}$} & \multirow{2}{*}{\multicolumn{2}{|c|}{$\begin{array}{c}\text { Risk Ratio } \\
\text { M-H, Random, } 95 \% \mathrm{Cl}\end{array}$}} \\
\hline & Events & Total & Events & Total & & & & \\
\hline Day 2013 & 24 & 301 & 23 & 295 & $32.9 \%$ & $1.02[0.59,1.77]$ & & \\
\hline Rouland 2013 & 16 & 213 & 36 & 189 & $32.6 \%$ & $0.39[0.23,0.69]$ & & \\
\hline Shedden 2010 & 21 & 131 & 28 & 130 & $34.5 \%$ & $0.74[0.45,1.24]$ & & \\
\hline Total $(95 \% \mathrm{Cl})$ & & 645 & & 614 & $100.0 \%$ & $0.67[0.39,1.15]$ & & \\
\hline Total events & 61 & & 87 & & & & & \\
\hline $\begin{array}{l}\text { Heterogeneity: } \mathrm{Tau}^{2}= \\
\text { Test for overall effect: }\end{array}$ & $\begin{array}{l}0.15 ; \mathrm{Chi}^{2}=5.94 \\
Z=1.46(P=0.15\end{array}$ & $=0.05)$ & $z^{2}=66 \%$ & & & & $\begin{array}{lc} & 1 \\
0.02 & 0.1 \\
\text { Fármacos sin conservantes }\end{array}$ & $\begin{array}{ccc}1 & 1 & 50 \\
1 & 10 & 50 \\
\text { Fármacos con conservantes }\end{array}$ \\
\hline
\end{tabular}




\section{Suspensión del tratamiento por efectos adversos}

\begin{tabular}{|c|c|c|c|c|c|c|c|c|}
\hline \multirow[b]{2}{*}{ Study or Subgroup } & \multicolumn{2}{|c|}{ Fármacos sin conservantes } & \multicolumn{2}{|c|}{ Fármacos con conservantes } & & \multirow{2}{*}{$\begin{array}{c}\text { Risk Ratio } \\
\text { M-H, Random, } 95 \% \mathrm{Cl} \\
\end{array}$} & \multirow{2}{*}{\multicolumn{2}{|c|}{$\begin{array}{c}\text { Risk Ratio } \\
\text { M-H, Random, } 95 \% \text { CI }\end{array}$}} \\
\hline & Events & Total & Events & Total & Weight & & & \\
\hline Day 2013 & 2 & 301 & 3 & 295 & $20.2 \%$ & $0.65[0.11,3.88]$ & - & \\
\hline Lewis 2007 & 5 & 344 & 4 & 346 & $37.7 \%$ & $1.26[0.34,4.64]$ & & 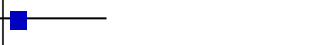 \\
\hline Rouland 2013 & 3 & 213 & 1 & 189 & $12.6 \%$ & $2.66[0.28,25.37]$ & & \\
\hline Shedden 2010 & 4 & 131 & 3 & 130 & $29.5 \%$ & $1.32[0.30,5.80]$ & & \\
\hline Total $(95 \% \mathrm{Cl})$ & & 989 & & 960 & $100.0 \%$ & $1.23[0.55,2.74]$ & & \\
\hline Total events & 14 & & 11 & & & & & \\
\hline $\begin{array}{l}\text { Heterogeneity: } \text { Tau }^{2}= \\
\text { Test for overall effect: }\end{array}$ & $\begin{array}{l}.00 ; \text { Chi }^{2}=0.95, \mathrm{~d} \\
=0.50(P=0.61)\end{array}$ & & & & & & $\begin{array}{l}0.01 \quad 0.1 \\
\text { Fármacos sin conservante }\end{array}$ & $\begin{array}{l}10 \\
100 \\
\text { Fármacos con conservante }\end{array}$ \\
\hline
\end{tabular}

\section{Queratitis puntiforme}

\begin{tabular}{|c|c|c|c|c|c|c|c|c|}
\hline \multirow[b]{2}{*}{ Study or Subgroup } & \multicolumn{2}{|c|}{ Fármacos sin conservantes } & \multicolumn{2}{|c|}{ Fármacos con conservantes } & \multicolumn{2}{|r|}{ Risk Ratio } & \multirow{2}{*}{\multicolumn{2}{|c|}{$\begin{array}{c}\text { Risk Ratio } \\
\text { M-H, Fixed, 95\% Cl }\end{array}$}} \\
\hline & Events & Total & Events & Total & Weight & M-H, Fixed, $95 \% \mathrm{Cl}$ & & \\
\hline Day 2013 & 9 & 301 & 9 & 295 & $22.6 \%$ & $0.98[0.39,2.43]$ & & \\
\hline Shedden 2010 & 22 & 131 & 31 & 130 & $77.4 \%$ & $0.70[0.43,1.15]$ & & \\
\hline Total $(95 \% \mathrm{Cl})$ & & 432 & & 425 & $100.0 \%$ & $0.77[0.50,1.18]$ & & \\
\hline Total events & 31 & & 40 & & & & & \\
\hline $\begin{array}{l}\text { Heterogeneity: } \mathrm{Chi}^{2}= \\
\text { Test for overall effect: }\end{array}$ & $\begin{array}{l}40, \mathrm{df}=1(\mathrm{P}=0 . \\
=1.21(P=0.23\end{array}$ & & & & & & $\begin{array}{cc}0.01 & 0.1 \\
\text { avours [experimental] }\end{array}$ & $\begin{array}{|cc|} & 1 \\
1 & 100 \\
\text { Favours [control] }\end{array}$ \\
\hline
\end{tabular}


4.3. Tratamiento con láser del glaucoma de ángullo abierto

4.3.1. Uso de diferentes tipos de láser en el tratamiento del glaucoma de ángulo abierto

4.3.1.1. Uso de láser diodo respecto al láser argón

Question: Diode laser trabeculoplasty versus argon laser trabeculoplasty for open angle

\section{Bibliography:}

Blyth CPJ, Moriarty AP, McHugh JDA. Diode laser trabeculoplasty versus argon laser trabeculoplasty in the control of primary open angle glaucoma. Lasers in Medical Science 1999; 14(2): 105-8.

Brancato R, Carassa R, Trabucchi G. Diode laser compared with argon laser for trabeculoplasty. American Journal of Ophthalmology 1991; 112(1): 50-5.

Chung PY, Schuman JS, Netland PA, Lloyd-Muhammad RA, Jacobs DS. Five-year results of a randomized, prospective, clinical trial of diode vs argon laser trabeculoplasty for open-angle glaucoma. American Journal of Ophthalmology 1998; 126(2): 185-90.

\begin{tabular}{|c|c|c|c|c|c|c|c|c|c|c|c|c|}
\hline \multicolumn{7}{|c|}{ Quality assessment } & \multicolumn{2}{|c|}{ No of patients } & \multicolumn{2}{|c|}{ Effect } & \multirow{2}{*}{ Quality } & \multirow{2}{*}{ Importance } \\
\hline \begin{tabular}{|c|} 
No of \\
studies
\end{tabular} & Design & $\begin{array}{c}\text { Risk of } \\
\text { bias }\end{array}$ & Inconsistency & Indirectness & Imprecision & $\begin{array}{c}\text { Other } \\
\text { considerations }\end{array}$ & \begin{tabular}{|c|} 
Diode laser \\
trabeculoplasty
\end{tabular} & $\begin{array}{c}\text { Argon laser } \\
\text { trabeculoplasty }\end{array}$ & $\begin{array}{l}\text { Relative } \\
(95 \% \mathrm{Cl})\end{array}$ & ) Absolute & & \\
\hline \multicolumn{13}{|c|}{ IOP control at 6 months } \\
\hline 2 & $\begin{array}{l}\text { randomisec } \\
\text { trials }\end{array}$ & $\begin{array}{l}\text { no } \\
\text { serious } \\
\text { risk of } \\
\text { bias }\end{array}$ & $\begin{array}{l}\text { no serious } \\
\text { inconsistency }\end{array}$ & serious $^{1}$ & $\begin{array}{l}\text { very } \\
\text { serious }^{2}\end{array}$ & none & $\begin{array}{c}28 / 30 \\
(93.3 \%)\end{array}$ & $\begin{array}{l}27 / 30 \\
(90 \%)\end{array}$ & \begin{tabular}{|c|}
$\mathrm{RR}$ \\
1.04 \\
$(0.88$ \\
to \\
$1.22)$ \\
\\
\end{tabular} & $\begin{array}{c}36 \text { more } \\
\text { per } \\
1.000 \\
\text { (from } \\
108 \\
\text { fewer to } \\
198 \\
\text { more) }\end{array}$ & $\begin{array}{l}\text { VERY } \\
\text { LOW }\end{array}$ & CRITICAL \\
\hline
\end{tabular}




\begin{tabular}{|c|c|c|c|c|c|c|c|c|c|c|c|c|}
\hline \multicolumn{13}{|c|}{ IOP control at 12 months } \\
\hline 2 & $\begin{array}{l}\text { randomised } \\
\text { trials }\end{array}$ & \begin{tabular}{l|l} 
no \\
serious \\
risk of \\
bias
\end{tabular} & $\mid \begin{array}{l}\text { no serious } \\
\text { inconsistency }\end{array}$ & serious $^{1}$ & \begin{tabular}{|l} 
very \\
serious
\end{tabular} & none & $\begin{array}{c}28 / 30 \\
(93.3 \%)\end{array}$ & $\begin{array}{c}25 / 30 \\
(83.3 \%)\end{array}$ & $\begin{array}{c}\mathrm{RR} \\
1.12 \\
(0.92 \\
\text { to } \\
1.35) \\
\end{array}$ & \begin{tabular}{|c|}
100 \\
more \\
per \\
1.000 \\
(from 67 \\
fewer to \\
292 \\
more)
\end{tabular} & \begin{tabular}{|l|} 
VER० \\
VERY \\
LOW
\end{tabular} & CRITICAL \\
\hline \multicolumn{13}{|c|}{ IOP control at 2 years } \\
\hline 1 & $\begin{array}{l}\text { randomised } \\
\text { trials }\end{array}$ & \begin{tabular}{l|l} 
no & $r$ \\
serious & i \\
risk of \\
bias
\end{tabular} & $\begin{array}{l}\text { no serious } \\
\text { inconsistency }\end{array}$ & serious $^{1}$ & \begin{tabular}{|l} 
very \\
serious
\end{tabular} & none & $\begin{array}{l}18 / 20 \\
(90 \%)\end{array}$ & $\begin{array}{l}16 / 20 \\
(80 \%)\end{array}$ & \begin{tabular}{|c|}
$\mathrm{RR}$ \\
1.13 \\
$(0.86$ \\
to \\
$1.46)$
\end{tabular} & \begin{tabular}{|c|}
104 \\
more \\
per \\
1.000 \\
(from \\
112 \\
fewer to \\
368 \\
more)
\end{tabular} & \begin{tabular}{|l|} 
पिएव \\
VERY \\
LOW
\end{tabular} & CRITICAL \\
\hline \multicolumn{13}{|c|}{ Adverse effects: peripheral anterior synechiae (PAS) formation } \\
\hline 3 & $\begin{array}{l}\text { randomised } \\
\text { trials }\end{array}$ & $\begin{array}{l}\text { no } \\
\text { serious } \\
\text { risk of } \\
\text { bias }\end{array}$ & serious $^{3}$ & $\begin{array}{l}\text { no serious } \\
\text { indirectness }\end{array}$ & $\begin{array}{l}\text { very } \\
\text { serious }{ }^{2}\end{array}$ & none & $\begin{array}{c}3 / 52 \\
(5.8 \%)\end{array}$ & $\begin{array}{c}7 / 58 \\
(12.1 \%)\end{array}$ & \begin{tabular}{|c|}
$\mathrm{RR}$ \\
0.50 \\
$(0.04$ \\
to \\
$5.77)$
\end{tabular} & \begin{tabular}{|c|}
60 \\
fewer \\
per \\
1.000 \\
(from \\
116 \\
fewer to \\
576 \\
more)
\end{tabular} & \begin{tabular}{|l|} 
पिएव \\
VERY \\
LOW
\end{tabular} & CRITICAL \\
\hline
\end{tabular}


Adverse effects: early intraocular pressure spikes

\begin{tabular}{|c|c|c|c|c|c|c|c|c|c|c|c|}
\hline 3 & \begin{tabular}{|l|l|}
$\begin{array}{l}\text { randomised } \\
\text { trials }\end{array}$ & $\begin{array}{l}\text { serious } \\
\text { risk of } \\
\text { bias }\end{array}$ \\
\end{tabular} & $\begin{array}{l}\text { no serious } \\
\text { inconsistency }\end{array}$ & serious ${ }^{4}$ & $\begin{array}{l}\text { very } \\
\text { serious }^{2}\end{array}$ & none & $\begin{array}{c}4 / 52 \\
(7.7 \%)\end{array}$ & $\begin{array}{c}7 / 58 \\
(12.1 \%)\end{array}$ & $\begin{array}{c}\text { RR } \\
0.66 \\
(0.21 \\
\text { to } \\
2.14) \\
\end{array}$ & \begin{tabular}{|c}
41 \\
fewer \\
per \\
1.000 \\
(from 95 \\
fewer to \\
138 \\
more)
\end{tabular} & \begin{tabular}{|l|} 
GQपि \\
VERY \\
LOW
\end{tabular} & CRITICAL \\
\hline
\end{tabular}

1 OAG progression as inferred from IOP.

${ }^{2}$ The $95 \%$ confidence shows appreciable benefit or appreciable harm. Low total number of events

${ }^{3} \mathrm{I}$-squared value of $59 \%$ indicates high statistical heterogeneity.

${ }^{4}$ Early intraocular pressure spikes inferred as likely to develop cataracts. 


\section{MÉTODOS}

Como medida del efecto, para las variables dicotómicas se utilizó el cociente de riesgos (CR) con intervalo de confianza del 95\%. Como método estadístico se utilizaron, respectivamente, los métodos de Mantel-Haenszel y de la varianza inversa. Para el metanálisis se utilizó el modelo de efectos fijos, excepto cuando se encontró heterogeneidad significativa; en ese caso, se utilizó el modelo de efectos aleatorios.

FOREST PLOTS

\section{Control de la PIO a los 6 meses}

\begin{tabular}{|c|c|c|c|c|c|c|c|}
\hline \multirow[b]{2}{*}{ Study or Subgroup } & \multicolumn{2}{|c|}{ DLT } & \multicolumn{2}{|c|}{ ALT } & \multirow[b]{2}{*}{ Weight } & \multirow{2}{*}{$\begin{array}{c}\text { Risk Ratio } \\
\text { M-H. Random. 95\% CI }\end{array}$} & \multirow{2}{*}{$\begin{array}{c}\text { Risk Ratio } \\
\text { M-H, Random, } 95 \% \mathrm{Cl}\end{array}$} \\
\hline & Events & Total & Events & Total & & & \\
\hline Blyth, 1999 & 18 & 20 & 18 & 20 & $62.7 \%$ & $1.00[0.81,1.23]$ & 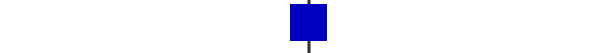 \\
\hline Brancato, 1991 & 10 & 10 & 9 & 10 & $37.3 \%$ & $1.11[0.85,1.44]$ & F \\
\hline Total $(95 \% \mathrm{Cl})$ & & 30 & & 30 & $100.0 \%$ & $1.04[0.88,1.22]$ & 9 \\
\hline Total events & 28 & & 27 & & & & \\
\hline $\begin{array}{l}\text { Heterogeneity: } \text { Tau }^{2}= \\
\text { Test for overall effect: }\end{array}$ & $\begin{array}{l}.00 ; \mathrm{Chi}^{2} \\
=0.45(\end{array}$ & $\begin{array}{l}=0.34 \\
D=0.6\end{array}$ & df $=1(F$ & $=0.56$ & ); $\left.\right|^{2}=0 \%$ & & $\begin{array}{cccc}0.005 & 0.1 & 1 & 10 \\
& \text { Favours DLT } & \text { Favours ALT }\end{array}$ \\
\hline
\end{tabular}


Control de la PIO a los 12 meses

\begin{tabular}{|c|c|c|c|c|c|c|c|}
\hline \multirow[b]{2}{*}{ Study or Subgroup } & \multicolumn{2}{|c|}{ DLT } & \multicolumn{2}{|c|}{ ALT } & \multirow[b]{2}{*}{ Weight } & \multirow{2}{*}{$\begin{array}{c}\text { Risk Ratio } \\
\text { M-H, Random, 95\% Cl }\end{array}$} & \multirow{2}{*}{$\begin{array}{c}\text { Risk Ratio } \\
\text { M-H, Random, } 95 \% \mathrm{Cl}\end{array}$} \\
\hline & Events & Total & Events & Total & & & \\
\hline Blyth, 1999 & 18 & 20 & 16 & 20 & $50.9 \%$ & $1.13[0.86,1.46]$ & $I$ \\
\hline Brancato, 1991 & 10 & 10 & 9 & 10 & $49.1 \%$ & $1.11[0.85,1.44]$ & 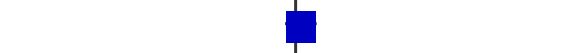 \\
\hline Total $(95 \% \mathrm{Cl})$ & & 30 & & 30 & $100.0 \%$ & $1.12[0.92,1.35]$ & 1 \\
\hline Total events & 28 & & 25 & & & & \\
\hline $\begin{array}{l}\text { Heterogeneity: Tau² } \\
\text { Test for overall effect }\end{array}$ & $\begin{array}{l}0.00 ; \mathrm{Chi}^{2} \\
z=1.14(\end{array}$ & $\begin{array}{l}=0.01 \\
=0.2\end{array}$ & $\mathrm{df}=1(\mathrm{P}$ & $=0.92$ & ); $\left.\right|^{2}=0 \%$ & & $\begin{array}{cccc}0.005 & 0.1 & 1 & 10 \\
& \text { Favours DLT } & \text { Favours ALT }\end{array}$ \\
\hline
\end{tabular}

\section{Control de la PIO a los 2 años}

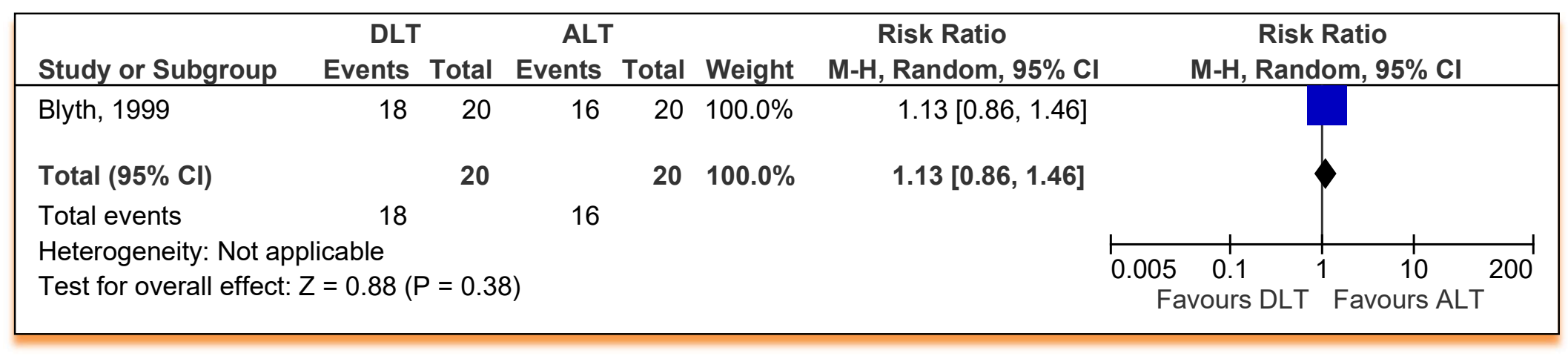


Efectos adversos: primeros picos hipertensivos intraoculares

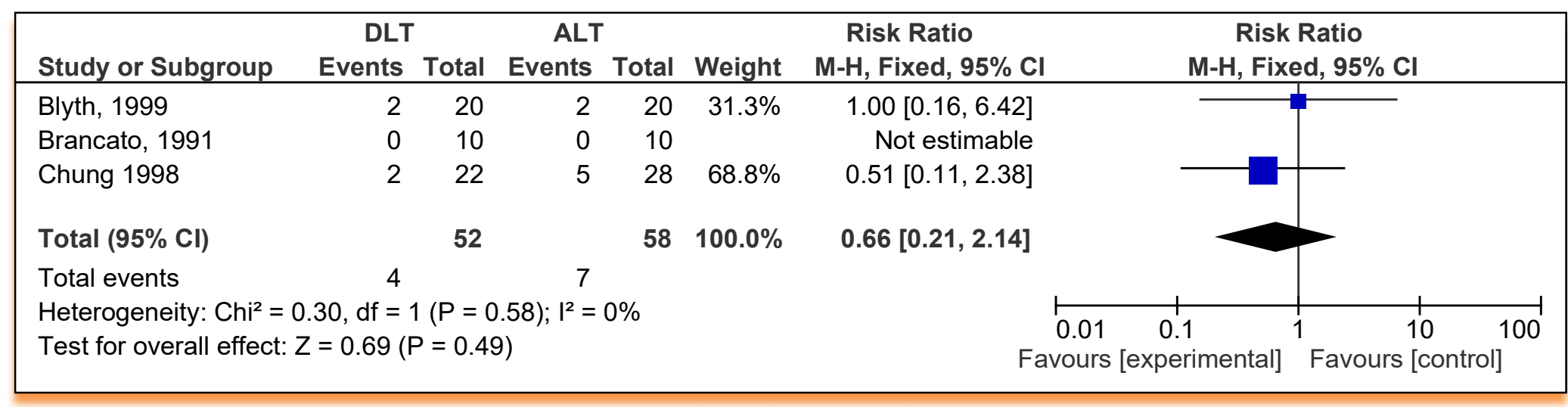

Formación de sinequias anteriores periféricas (SAP)

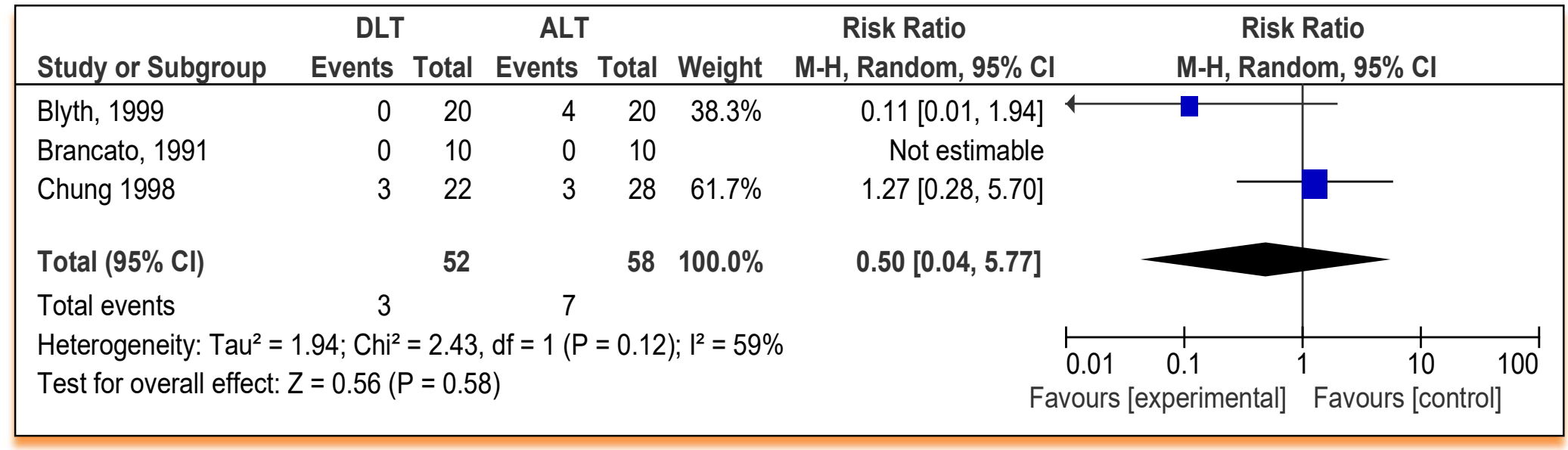


4.3.1.2. Uso de láser selectivo respecto al láser argon

Question: Should Selective laser trabeculoplasty (Nd: Yag laser) vs argon laser trabeculoplasty be used for open angle glaucoma [Data only. When citing this record quote "Cochrane Database of Systematic Reviews 2007, Issue 4".]?

\section{Bibliography:}

Wang H, Cheng JW, Wei RL, Cai JP, Li Y, Ma XY. Meta-analysis of selective laser trabeculoplasty with argon laser trabeculoplasty in the treatment of open-angle glaucoma. Can J Ophthalmol. 2013; 48(3): 186-92.

Kent SS, Hutnik CM, Birt CM, Damji KF, Harasymowycz P et al. (2013) A Randomized Clinical Trial of Selective Laser Trabeculoplasty Versus Argon Laser Trabeculoplasty in Patients With Pseudoexfoliation. J Glaucoma.

Rosenfeld E, Shemesh G, Kurtz S (2012) The efficacy of selective laser trabeculoplasty versus argon laser trabeculoplasty in pseudophakic glaucoma patients. Clin. Ophthalmol 6: 1935-1940.

\begin{tabular}{|c|c|c|c|c|c|c|c|c|c|c|c|c|}
\hline \multicolumn{7}{|c|}{ Quality assessment } & \multicolumn{2}{|c|}{ No of patients } & \multicolumn{2}{|c|}{ Effect } & \multirow[b]{2}{*}{ Quality } & \multirow{2}{*}{$\begin{array}{c}\text { Importanc } \\
e\end{array}$} \\
\hline $\begin{array}{c}\text { No of } \\
\text { studie } \\
\mathbf{s}\end{array} \mid$ & Design & $\begin{array}{c}\text { Risk of } \\
\text { bias }\end{array}$ & Inconsistency & Indirectness & Imprecision & $\begin{array}{c}\text { Other } \\
\text { consideration } \\
\text { s }\end{array}$ & \begin{tabular}{|c} 
Selective \\
laser \\
trabeculoplas \\
y (Nd: Yag \\
laser)
\end{tabular} & $\begin{array}{c}\text { Argon laser } \\
\text { trabeculoplast } \\
y\end{array}$ & $\begin{array}{l}\text { Relative } \\
(95 \% \mathrm{Cl})\end{array}$ & $\begin{array}{c}\text { Absolut } \\
\mathrm{e}\end{array}$ & & \\
\hline \multicolumn{13}{|c|}{ IOP reduction (all patients) (better indicated by lower values) } \\
\hline 9 & $\begin{array}{l}\text { randomise } \\
\text { d trials }\end{array}$ & $\begin{array}{l}\text { no } \\
\text { serious } \\
\text { risk of } \\
\text { bias }\end{array}$ & \begin{tabular}{|l|} 
no serious \\
inconsistenc \\
$y$
\end{tabular} & serious $^{1}$ & $\begin{array}{l}\text { no serious } \\
\text { imprecisio } \\
\text { n }\end{array}$ & none & 283 & 276 & - & \begin{tabular}{|c|} 
MD \\
0.69 \\
higher \\
$(0.22$ \\
to 1.15 \\
higher $)$ \\
2
\end{tabular} & $\begin{array}{c}\text { MODER } \\
\mathrm{E} \\
\end{array}$ & $\begin{array}{c}\text { CRITICA } \\
L\end{array}$ \\
\hline
\end{tabular}




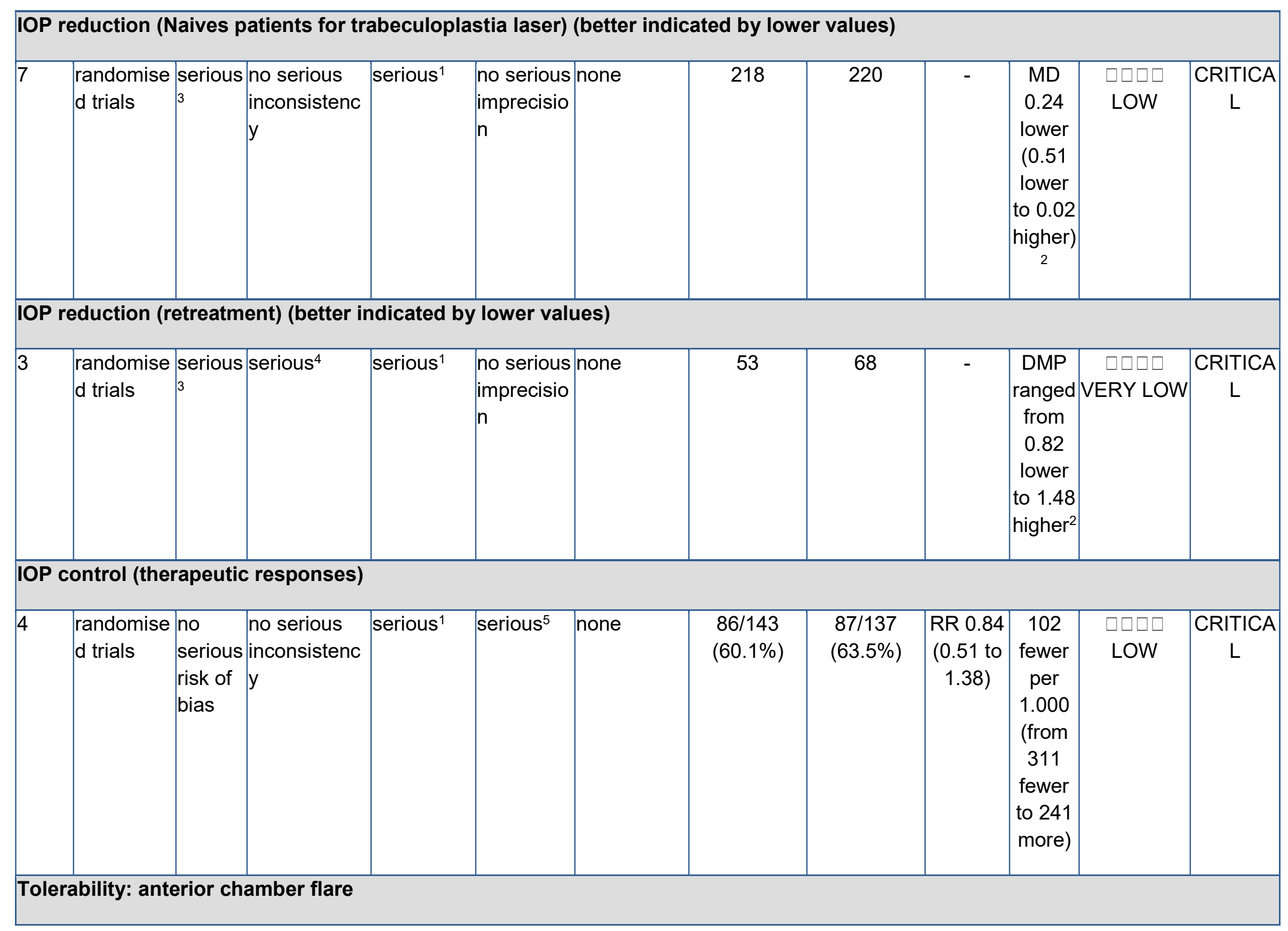




\begin{tabular}{|c|c|c|c|c|c|c|c|c|c|c|c|c|}
\hline 2 & $\begin{array}{l}\text { randomise } \\
\mathrm{d} \text { trials }\end{array}$ & $\begin{array}{l}\text { no } \\
\text { serious } \\
\text { risk of } \\
\text { bias }^{6}\end{array}$ & $\begin{array}{l}\text { no serious } \\
\text { inconsistenc } \\
y^{6}\end{array}$ & $\begin{array}{l}\text { no serious } \\
\text { indirectnes } \\
\text { s }\end{array}$ & serious ${ }^{5}$ & none & - & - & $\left|\begin{array}{c}\text { Not } \\
\text { estimabl } \\
\mathrm{e}\end{array}\right|$ & - & $\begin{array}{c}\text { MODER } \\
\text { MORAT } \\
\text { E }\end{array}$ & $\begin{array}{c}\text { CRITICA } \\
L\end{array}$ \\
\hline \multicolumn{13}{|c|}{ Tolerability: IOP spike } \\
\hline 3 & \begin{tabular}{|l|} 
randomise \\
$\mathrm{d}$ trials
\end{tabular} & $\begin{array}{l}\text { no } \\
\text { serious } \\
\text { risk of } \\
\text { bias }^{6}\end{array}$ & $\begin{array}{l}\text { no serious } \\
\text { inconsistenc } \\
y^{6}\end{array}$ & serious $^{7}$ & serious $^{5}$ & none & - & - & $\left|\begin{array}{c}\text { Not } \\
\text { estimabl } \\
e\end{array}\right|$ & - & $\begin{array}{l}\text { पिपा } \\
\text { LOW }\end{array}$ & $\begin{array}{c}\text { CRITICA } \\
L\end{array}$ \\
\hline
\end{tabular}

OAG progression as inferred from IOP.

Measured by $\mathrm{mm} \mathrm{Hg}$

${ }^{3}$ The SR of Wang 2013 take data for sensitivity analysis from studies in wich the subgroup analysis (retreatment or naive patients) was made after the randomisation.

${ }^{4}$ Pool data analysis not performed because detailed information of individual studies were not available. However, the direction of the estimates was opposite, inferring inconsistency.

${ }^{5}$ Large confidence interval limits precision: appreciable benefit or appreciable harm.

${ }^{6}$ No data provided, but none of the studies included had serious limitations.

${ }^{7}$ Early intraocular pressure spikes inferred as likely to develop cataracts. 


\section{MÉTODOS}

Como medida del efecto, para las variables dicotómicas se utilizó el cociente de riesgos (CR) con intervalo de confianza del 95\%. Como método estadístico se utilizaron, respectivamente, los métodos de Mantel-Haenszel y de la varianza inversa. Para el metanálisis se utilizó el modelo de efectos fijos, excepto cuando se encontró heterogeneidad significativa; en ese caso, se utilizó el modelo de efectos aleatorios.

\section{FOREST PLOTS}

\section{Reducción de PIO}

Láser selectivo frente a láser argón (todos los pacientes).

\begin{tabular}{|c|c|c|c|c|c|c|c|c|c|c|c|}
\hline \multirow[b]{2}{*}{ Stucty or Subgroup } & \multicolumn{3}{|c|}{ Láser selectivo } & \multicolumn{3}{|c|}{ Láser argón } & \multicolumn{3}{|c|}{ Mean Difference } & \multirow{2}{*}{\multicolumn{2}{|c|}{$\begin{array}{l}\text { Mean Difference } \\
\mathrm{N}, \text { Fixed, 95\% } \mathrm{Cl}\end{array}$}} \\
\hline & Mean & SD & Total & Mean & SD & Total & Weight & $\mathrm{N}$, Fixed, $95 \% \mathrm{Cl}$ & Year & & \\
\hline Casa 2004 & 5.4 & 6.3 & 20 & 4.6 & 5 & 20 & $1.7 \%$ & $0.80[-2.72,4.32]$ & 2004 & & \\
\hline Damii 2006 & 5.8 & 6.2 & 73 & 6 & 4.8 & 74 & $6.7 \%$ & $-0.20[-1.99,1.59]$ & 2006 & & \\
\hline Birt 2007 & 5.2 & 5.8 & 30 & 5.4 & 6.4 & 39 & $2.6 \%$ & $-0.20[-3.09,2.69]$ & 2007 & & \\
\hline Russo 2009 (Group A) & 6 & 2.08 & 43 & 6.1 & 2.2 & 41 & $25.7 \%$ & $-0.10[-1.02,0.82]$ & 2009 & & \\
\hline Russo 2009 (Group B) & 6.24 & 1.6 & 18 & 4.65 & 1.1 & 18 & $26.8 \%$ & $1.59[0.69,2.49]$ & 2009 & & - \\
\hline Liu 2011 & 1.8 & 5.8 & 20 & 2.8 & 7.2 & 22 & $1.4 \%$ & $-1.00[-4.94,2.94]$ & 2011 & & \\
\hline Júnior 2011 & 5.1 & 2.5 & 25 & 4.4 & 2.8 & 20 & $8.7 \%$ & $0.70[-0.87,2.27]$ & 2011 & & \\
\hline Rosenfeld 2012 & 4.3 & 1.33 & 17 & 3.23 & 1.42 & 16 & $24.4 \%$ & $1.07[0.13,2.01]$ & 2012 & & - \\
\hline Kent 2013 & 6.8 & 5.4 & 37 & 7.7 & 7.12 & 26 & $2.0 \%$ & $-0.90[-4.14,2.34]$ & 2013 & & \\
\hline Total $\left(95^{\circ} \mathrm{Cl}\right)$ & & & 283 & & & 276 & $100.0 \%$ & $0.69[0.22,1.15]$ & & & 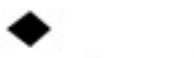 \\
\hline $\begin{array}{l}\text { Heterogeneity: } \mathrm{Ch}^{2}=10 \\
\text { Test for overall effect: } z\end{array}$ & $\begin{array}{l}29, \mathrm{df}= \\
2.89(\mathrm{P}\end{array}$ & $\begin{array}{l}(P=0 \\
0.00\end{array}$ & $25) ; 1^{2}=$ & & & & & & & $\begin{array}{ccc}1 & 1 & 1 \\
-4 & -2 & 0 \\
\text { Láser selectivo }\end{array}$ & $\begin{array}{c}\frac{1}{2} \\
\text { Láser arg }\end{array}$ \\
\hline
\end{tabular}




\section{Reducción de PIO}

Láser selectivo frente a láser argón (pacientes no tratados previamente con trabeculoplastia láser)

\begin{tabular}{|c|c|c|c|c|c|c|c|c|c|c|}
\hline \multirow[b]{2}{*}{ Study or Subgroup } & \multicolumn{3}{|c|}{ Láser selectivo } & \multicolumn{3}{|c|}{ Láser argón } & \multicolumn{3}{|c|}{ Mean Difference } & \multirow{2}{*}{$\begin{array}{l}\text { Mean Difference } \\
\mathrm{N}, \text { Fixed, } 95^{\circ} \mathrm{Cl}\end{array}$} \\
\hline & Mean & SD & Total & Mean & SD & Total & Weight & N, Fixed, 95\% Cl & Year & \\
\hline Casa 2004 & 5.4 & 6.3 & 20 & 4.6 & 5 & 20 & $0.6 \%$ & $0.80[-2.72,4.32]$ & 2004 & \\
\hline Damij 2006 & 5.7 & 0.7 & 54 & 6 & 0.7 & 41 & $86.4 \%$ & $-0.30[-0.58,-0.02]$ & 2006 & \\
\hline Birt 2007 & 5.2 & 5.8 & 30 & 5.4 & 6.4 & 39 & $0.8 \%$ & $-0.20[-3.09,2.69]$ & 2007 & \\
\hline Russo 2009 (Group A) & 6 & 2.08 & 43 & 6.1 & 2.2 & 41 & $8.3 \%$ & $-0.10[-1.02,0.82]$ & 2009 & \\
\hline Liu 2011 & 1.8 & 5.8 & 20 & 2.8 & 7.2 & 22 & $0.4 \%$ & $-1.00[-4.94,2.94]$ & 2011 & \\
\hline Júnior 2011 & 5.1 & 2.5 & 25 & 4.4 & 2.8 & 20 & $2.8 \%$ & $0.70[-0.87,2.27]$ & 2011 & \\
\hline Kent 2013 & 7.7 & 7.12 & 26 & 6.8 & 5.4 & 37 & $0.7 \%$ & $0.90[-2.34,4.14]$ & 2013 & \\
\hline \multicolumn{3}{|l|}{ Total $(95 \%$ Cl) } & 218 & & & 220 & $100.0 \%$ & $-0.24[-0.51,0.02]$ & & 1 \\
\hline \multicolumn{7}{|c|}{$\begin{array}{l}\text { Heterogeneity: } \mathrm{Ch}^{2}=2.59, \mathrm{df}=6(P=0.86) ; \mathrm{I}^{2}=0 \% \\
\text { Test for overall effect: } Z=1.81(P=0.07)\end{array}$} & & & & $\begin{array}{ccccc} & 1 & 1 & 1 & 1 \\
-4 & -2 & 0 & 2 & 4 \\
\text { Láser selectivo } & \text { Láser argór }\end{array}$ \\
\hline
\end{tabular}


4.3.2. Trabeculoplastia láser respecto al tratamiento farmacológico en el glaucoma de ángulo abierto Question: Should argon laser trabeculoplasty vs medical treatment be used for open angle glaucoma [Data only. When citing this record quote "Cochrane Database of Systematic Reviews 2007, Issue 4".]?

\section{Bibliography:}

Rolim de Moura CR, Paranhos Jr A, Wormald R. Laser trabeculoplasty for open angle glaucoma. "Cochrane Database of Systematic Reviews 2007, Issue 4".

\begin{tabular}{|c|c|c|c|c|c|c|c|c|c|c|c|c|}
\hline \multicolumn{7}{|c|}{ Quality assessment } & \multicolumn{2}{|c|}{ No of patients } & \multicolumn{2}{|c|}{ Effect } & \multirow{2}{*}{ Quality } & \multirow{2}{*}{ Importance } \\
\hline \begin{tabular}{|c|} 
No of \\
studies
\end{tabular} & Design & $\begin{array}{c}\text { Risk of } \\
\text { bias }\end{array}$ & Inconsistency & Indirectness & Imprecision & $\begin{array}{c}\text { Other } \\
\text { considerations }\end{array}$ & $\begin{array}{l}\text { argon laser } \\
\text { trabeculoplasty }\end{array}$ & \begin{tabular}{|c|} 
medical \\
treatment
\end{tabular} & $\begin{array}{c}\text { Relative } \\
(95 \% \\
\mathrm{Cl})\end{array}$ & Absolute & & \\
\hline \multicolumn{13}{|c|}{ Failure to control IOP-at 6 months } \\
\hline 2 & $\begin{array}{l}\text { randomise } \\
\text { trials }\end{array}$ & \begin{tabular}{|l|} 
no \\
serious \\
risk of \\
bias
\end{tabular} & $\mid \begin{array}{l}\text { no serious } \\
\text { inconsistency }\end{array}$ & serious $^{1}$ & $\begin{array}{l}\text { no serious } \\
\text { imprecision }\end{array}$ & none & $\begin{array}{c}38 / 311 \\
(12.2 \%)\end{array}$ & \begin{tabular}{|l|}
$107 / 313$ \\
$(34.2 \%)$
\end{tabular} & \begin{tabular}{|c|}
$R R$ \\
0.38 \\
$(0.24$ \\
to \\
$0.61)$
\end{tabular} & $\begin{array}{c}212 \\
\text { fewer } \\
\text { per } \\
1.000 \\
\text { (from } \\
133 \\
\text { fewer } \\
\text { to } 260 \\
\text { fewer) }\end{array}$ & $\begin{array}{c}\text { पिमा } \\
\text { MODERATE }\end{array}$ & CRITICAL \\
\hline \multicolumn{13}{|c|}{ Failure to control IOP-at 24 months } \\
\hline 3 & $\begin{array}{l}\text { randomise } \\
\text { trials }\end{array}$ & \begin{tabular}{|l|} 
no \\
serious \\
risk of \\
bias
\end{tabular} & $\begin{array}{l}\text { no serious } \\
\text { inconsistency }\end{array}$ & serious $^{1}$ & $\begin{array}{l}\text { no serious } \\
\text { imprecision }\end{array}$ & none & $\begin{array}{l}174 / 366 \\
(47.5 \%)\end{array}$ & \begin{tabular}{|l|}
$216 / 369$ \\
$(58.5 \%)$
\end{tabular} & $\begin{array}{c}\text { RR } 0.8 \\
(0.71 \\
\text { to } \\
0.91)\end{array}$ & $\begin{array}{c}117 \\
\text { fewer } \\
\text { per } \\
1.000 \\
\text { (from }\end{array}$ & $\begin{array}{c}\text { पIGI } \\
\text { MODERATE }\end{array}$ & \\
\hline
\end{tabular}




\begin{tabular}{|c|c|c|c|c|c|c|c|c|c|c|c|c|}
\hline & & & & & & & & & & $\begin{array}{c}53 \\
\text { fewer } \\
\text { to } 170 \\
\text { fewer) }\end{array}$ & & \\
\hline \multicolumn{13}{|c|}{ Visual field progression-at 24 months } \\
\hline 2 & $\begin{array}{l}\text { randomised } \\
\text { trials }\end{array}$ & $\begin{array}{l}\text { no } \\
\text { serious } \\
\text { risk of } \\
\text { bias }\end{array}$ & $\begin{array}{l}\text { no serious } \\
\text { inconsistency }\end{array}$ & $\mid \begin{array}{l}\text { no serious } \\
\text { indirectness }\end{array}$ & serious ${ }^{2}$ & none & $\begin{array}{l}23 / 311 \\
(7.4 \%)\end{array}$ & $\begin{array}{l}33 / 313 \\
(10.5 \%)\end{array}$ & $\begin{array}{c}\mathrm{RR} 0.7 \\
(0.42 \\
\text { to } \\
1.16)\end{array}$ & $\begin{array}{c}32 \\
\text { fewer } \\
\text { per } \\
1.000 \\
\text { (from } \\
61 \\
\text { fewer } \\
\text { to } 17 \\
\text { more) }\end{array}$ & 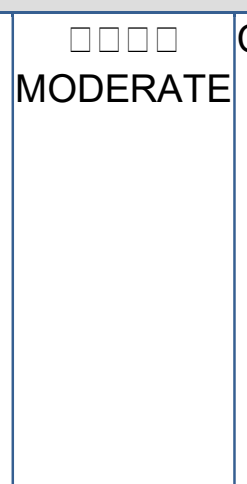 & CRITICAL \\
\hline \multicolumn{13}{|c|}{ Optic neuropathy progression-at 24 months } \\
\hline 2 & $\begin{array}{l}\text { randomised } \\
\text { trials }\end{array}$ & $\begin{array}{l}\text { no } \\
\text { serious } \\
\text { risk of } \\
\text { bias }\end{array}$ & $\begin{array}{l}\text { no serious } \\
\text { inconsistency }\end{array}$ & \begin{tabular}{|l} 
no serious \\
indirectness
\end{tabular} & serious $^{2}$ & none & $\begin{array}{l}22 / 311 \\
(7.1 \%)\end{array}$ & $\begin{array}{l}31 / 313 \\
(9.9 \%)\end{array}$ & \begin{tabular}{|c|}
$\mathrm{RR}$ \\
0.71 \\
$(0.38$ \\
to \\
$1.34)$
\end{tabular} & $\begin{array}{c}29 \\
\text { fewer } \\
\text { per } \\
1.000 \\
\text { (from } \\
61 \\
\text { fewer } \\
\text { to } 34 \\
\text { more) }\end{array}$ & 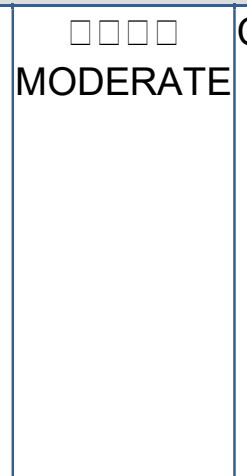 & CRITICAL \\
\hline \multicolumn{13}{|c|}{ Adverse effects: PAS formation } \\
\hline 2 & $\begin{array}{l}\text { randomised } \\
\text { trials }\end{array}$ & $\begin{array}{l}\text { no } \\
\text { serious } \\
\text { risk of } \\
\text { bias }\end{array}$ & $\begin{array}{l}\text { no serious } \\
\text { inconsistency }\end{array}$ & $\begin{array}{c}\text { no serious } \\
\text { indirectness }\end{array}$ & serious $^{2}$ & none & $\begin{array}{c}94 / 311 \\
(30.2 \%)\end{array}$ & \begin{tabular}{|c|}
$8 / 313$ \\
$(2.6 \%)$
\end{tabular} & \begin{tabular}{|c|}
$\mathrm{RR}$ \\
11.15 \\
$(5.63$ \\
to
\end{tabular} & $\begin{array}{c}259 \\
\text { more } \\
\text { per } \\
1.000\end{array}$ & 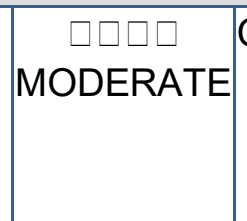 & CRITICAL \\
\hline
\end{tabular}




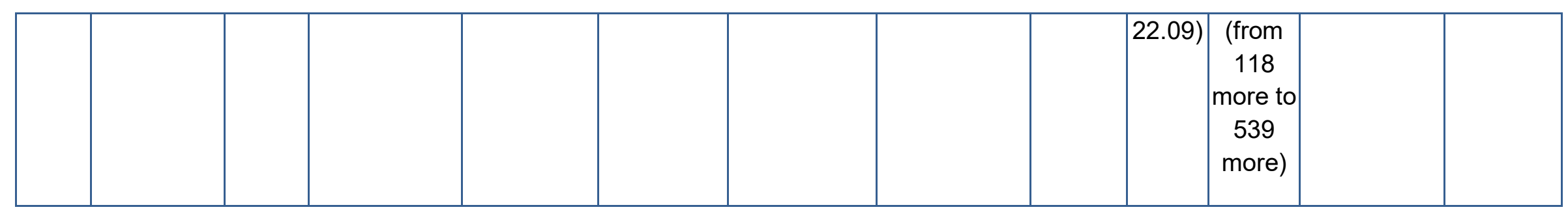

OAG progression as inferred from IOP.

${ }^{2}$ Low number of events.

Question: Should selective laser trabeculoplasty vs medical treatment be used for open-angle glaucoma (OAG) and ocular hypertension $(\mathrm{OHT})$ ?

Bibliography:

Nagar M, Luhishi E, Shah N. Intraocular pressure control and fluctuation: the effect of treatment with selective laser trabeculoplasty. $\mathrm{Br}$ Ophthalmol. 2009; 93(4): 497-501.

\begin{tabular}{|c|c|c|c|c|c|c|c|c|c|c|c|c|}
\hline \multicolumn{7}{|c|}{ Quality assessment } & \multicolumn{2}{|c|}{ No of patients } & \multicolumn{2}{|c|}{ Effect } & \multirow{2}{*}{ Quality } & \multirow{2}{*}{ Importance } \\
\hline $\begin{array}{l}\text { No of } \\
\text { studies }\end{array}$ & Design & $\begin{array}{c}\text { Risk of } \\
\text { bias }\end{array}$ & Inconsistency & Indirectness & Imprecision & $\begin{array}{c}\text { Other } \\
\text { considerations }\end{array}$ & \begin{tabular}{|c|} 
medical \\
treatment
\end{tabular} & $\begin{array}{c}\text { Selective laser } \\
\text { trabeculoplasty }\end{array}$ & $\begin{array}{l}\text { Relative } \\
(95 \% \mathrm{Cl})\end{array}$ & Absolute & & \\
\hline \multicolumn{13}{|c|}{ IOP control (1 month follow) } \\
\hline 1 & $\begin{array}{l}\text { randomisec } \\
\text { trials }\end{array}$ & $\begin{array}{l}\text { no } \\
\text { serious } \\
\text { risk of } \\
\text { bias }\end{array}$ & $\begin{array}{l}\text { no serious } \\
\text { inconsistency }\end{array}$ & serious ${ }^{1}$ & $\begin{array}{l}\text { very } \\
\text { serious }{ }^{2}\end{array}$ & none & $\begin{array}{l}13 / 20 \\
(65 \%)\end{array}$ & $\begin{array}{l}8 / 20 \\
(40 \%)\end{array}$ & \begin{tabular}{|c}
$\mathrm{RR}$ \\
6.21 \\
$(1.72$ to \\
$51.1)$
\end{tabular} & $\begin{array}{c}1.000 \\
\text { more per } \\
1.000 \\
\text { (from } 288 \\
\text { more to } \\
1.000 \\
\text { more) }\end{array}$ & $\begin{array}{l}\text { VID० } \\
\text { VERY } \\
\text { LOW }\end{array}$ & CRITICAL \\
\hline
\end{tabular}




IOP control (4-6 month follow)
\begin{tabular}{|l|l|l|l|l|l|l|l|l|l|l|}
\hline 1 & $\begin{array}{l}\text { randomised } \\
\text { trials }\end{array}$ & $\begin{array}{l}\text { no } \\
\text { serious } \\
\text { risk of } \\
\text { bias } \\
\text { inconsistency }\end{array}$ & no serious & serious & very \\
serious
\end{tabular}

1 OAG progression as inferred from IOP

${ }^{2}$ Low total number of events. Large confidence intervals limits precision: few or show appreciable benefit or appreciable harm.

4.3.3. Trabeculoplastia con láser asociada al tratamiento farmacológico respecto al tratamiento farmacológico en el glaucoma de ángulo abierto

Question: Should Comparison of laser trabeculoplasty plus pharmacological treatment between pharmacological treatment be used for primary open angle glaucoma?

Bibliography:

Moriarty BJ, Char JN, Acheson RW, Dunn DT. Argon laser trabeculoplasty in primary open-angle glaucoma - results in black Jamaican population. International Ophthalmology 1988; 12(4): 217-21.

Sherwood MB, Lattimer J, Hitchings RA. Laser trabeculoplasty as supplementary treatment for primary open angle glaucoma. British Journal of Ophthalmology 1987; 71(3)188: 91. 


\begin{tabular}{|c|c|c|c|c|c|c|c|c|c|c|c|c|}
\hline \multicolumn{7}{|c|}{ Quality assessment } & \multicolumn{2}{|c|}{ No of patients } & \multicolumn{2}{|c|}{ Effect } & \multirow[b]{2}{*}{ Quality } & \multirow[b]{2}{*}{ Importance } \\
\hline $\begin{array}{c}\text { No of } \\
\text { studies }\end{array}$ & Design & $\begin{array}{l}\text { Risk of } \\
\text { bias }\end{array}$ & Inconsistency & Indirectness & s Imprecision & $\begin{array}{c}\text { Other } \\
\text { considerations }\end{array}$ & $\begin{array}{c}\text { laser } \\
\text { trabeculoplasty } \\
\text { plus } \\
\text { pharmacological } \\
\text { treatment }\end{array}$ & $\begin{array}{l}\text { Pharmacological } \\
\text { treatment }\end{array}$ & $\begin{array}{l}\text { Relative } \\
(95 \% \mathrm{Cl})\end{array}$ & Absolute & & \\
\hline \multicolumn{13}{|c|}{ Control IOP } \\
\hline 2 & $\begin{array}{l}\text { randomisec } \\
\text { trials }\end{array}$ & serious $^{1}$ & serious ${ }^{2}$ & serious $^{3}$ & serious $^{4}$ & none & $\begin{array}{c}39 / 49 \\
(79.6 \%)\end{array}$ & $\begin{array}{c}5 / 46 \\
(10.9 \%)\end{array}$ & $\begin{array}{c}\mathrm{RR} \\
9.43 \\
(0.34 \text { to } \\
260.22) \\
\\
\end{array}$ & \begin{tabular}{|c|}
916 \\
more \\
per \\
1.000 \\
(from \\
72 \\
fewer to \\
1.000 \\
more)
\end{tabular} & $\begin{array}{l}\text { VDQ } \\
\text { VERY } \\
\text { LOW }\end{array}$ & CRITICAL \\
\hline
\end{tabular}

${ }^{1}$ Allocation concealment, randomisation methods and masking of outcome assessment are not reported in one study.

$2 \mathrm{I}$-squared value of $82 \%$ indicates high statistical heterogeneity which may have been due to the studies being from very different populations. One study is exclusively in Afro-Caribbean patients.

OAG progression as inferred from IOP.

${ }^{4}$ Small sample size/wide confidence interval that include potential benefit or potential harm. 


\section{MÉTODOS}

Como medida del efecto, para las variables dicotómicas se utilizó el cociente de riesgos (CR) con intervalo de confianza del 95\%. Como método estadístico se utilizaron, respectivamente, los métodos de Mantel-Haenszel y de la varianza inversa. Para el metanálisis se utilizó el modelo de efectos fijos, excepto cuando se encontró heterogeneidad significativa; en ese caso, se utilizó el modelo de efectos aleatorios.

FOREST PLOTS

\section{Control de la PIO a los 12 meses}

\begin{tabular}{|c|c|c|c|c|c|c|c|c|}
\hline \multirow[b]{2}{*}{ Study or Subgroup } & \multicolumn{2}{|c|}{ Láser+Fármaco } & \multicolumn{2}{|c|}{ Fármaco } & \multirow{3}{*}{$\frac{\text { Weight }}{57.6 \%}$} & $\begin{array}{l}\text { Risk Ratio } \\
\text { R }\end{array}$ & \multirow{2}{*}{\multicolumn{2}{|c|}{$\begin{array}{c}\text { Risk Ratio } \\
\text { M-H, Random, } 95 \% \mathrm{Cl}\end{array}$}} \\
\hline & Events & Total & Events & Total & & $\mathrm{M}-\mathrm{H}$, Random, $95 \% \mathrm{Cl}$ & & \\
\hline Moriarty, 1988 & 17 & 25 & 5 & 22 & & $2.99[1.32,6.77]$ & & E \\
\hline Sherwood 1987 & 22 & 24 & 0 & 24 & $42.4 \%$ & $45.00[2.89,701.85]$ & & \\
\hline Total $(95 \% \mathrm{Cl})$ & & 49 & & 46 & $100.0 \%$ & $9.43[0.34,260.22]$ & & \\
\hline Total events & 39 & & 5 & & & & & \\
\hline $\begin{array}{l}\text { Heterogeneity: } \mathrm{Tau}^{2}= \\
\text { Test for overall effect }\end{array}$ & $\begin{array}{l}.80 ; \mathrm{Chi}^{2}= \\
=1.33(\mathrm{P}\end{array}$ & $\begin{array}{l}49, \mathrm{df} \\
0.18)\end{array}$ & $=1(P=C$ & $.02) ; 1^{2}$ & $=82 \%$ & & $\begin{array}{cc}0.001 & 0.1 \\
\text { avours [experimental] }\end{array}$ & $\begin{array}{ccc}1 & 10 & 1000 \\
\text { Favours [control] }\end{array}$ \\
\hline
\end{tabular}


4.3.4. Trabeculoplastia con láser frente trabeculectomía en el tratamiento del glaucoma de ángulo abierto Question: Argon laser trabeculoplasty versus trabeculectomy for open angle glaucoma?

\section{Bibliography:}

Commissioned by the National Institute for Health and Clinical Excellence. Glaucoma Diagnosis and management of chronic open angle glaucoma and ocular hypertension. UK. 2009.

\begin{tabular}{|c|c|c|c|c|c|c|c|c|c|c|c|c|}
\hline \multicolumn{7}{|c|}{ Quality assessment } & \multicolumn{2}{|c|}{ No of patients } & \multicolumn{2}{|c|}{ Effect } & \multirow{2}{*}{ Quality } & \multirow{2}{*}{ Importance } \\
\hline \begin{tabular}{|c|} 
No of \\
studies
\end{tabular} & Design & $\begin{array}{c}\text { Risk of } \\
\text { bias }\end{array}$ & Inconsistency & Indirectness & Imprecision & $\begin{array}{c}\text { Other } \\
\text { considerations }\end{array}$ & $\begin{array}{c}\text { Argon laser } \\
\text { trabeculoplasty }\end{array}$ & trabeculectomy & $\begin{array}{c}\text { Relative } \\
(95 \% \\
\mathrm{Cl})\end{array}$ & Absolute & & \\
\hline \multicolumn{13}{|c|}{ Failure to control IOP-at 6 months } \\
\hline 2 & $\begin{array}{l}\text { randomise } \\
\text { trials }\end{array}$ & serious 1 & $\begin{array}{l}\text { no serious } \\
\text { inconsistency }\end{array}$ & serious $^{2}$ & serious $^{3}$ & none & $\begin{array}{l}34 / 419 \\
(8.1 \%)\end{array}$ & $\begin{array}{l}10 / 400 \\
(2.5 \%)\end{array}$ & \begin{tabular}{|c|}
$\mathrm{RR}$ \\
3.14 \\
$(1.6$ to \\
$6.18)$ \\
\\
\end{tabular} & $\begin{array}{c}54 \text { more } \\
\text { per } \\
1.000 \\
\text { (from } \\
15 \text { more } \\
\text { to } 130 \\
\text { more) }\end{array}$ & $\begin{array}{l}\text { VERY } \\
\text { LOW }\end{array}$ & CRITICAL \\
\hline \multicolumn{13}{|c|}{ Failure to control IOP-at 24 months } \\
\hline 2 & $\begin{array}{l}\text { randomise } \\
\text { trials }\end{array}$ & serious $^{1}$ & serious $^{4}$ & serious $^{2}$ & \begin{tabular}{|l|} 
no serious \\
imprecision
\end{tabular} & none & $\begin{array}{c}72 / 459 \\
(15.7 \%)\end{array}$ & $\begin{array}{l}34 / 442 \\
(7.7 \%)\end{array}$ & \begin{tabular}{|c|}
$\mathrm{RR}$ \\
2.03 \\
$(1.38$ \\
to \\
$2.98)$ \\
\end{tabular} & $\begin{array}{c}79 \text { more } \\
\text { per } \\
1.000 \\
\text { (from } \\
29 \text { more } \\
\text { to } 152 \\
\text { more) }\end{array}$ & $\begin{array}{l}\text { LET } \\
\text { VERY } \\
\text { LOW }\end{array}$ & CRITICAL \\
\hline
\end{tabular}


${ }^{1}$ One study does not resport masking of outcome assessment

OAG progression as inferred from IOP.

Low number of events. $95 \% \mathrm{Cl}$ does not exclude appreciable benefit or harm.

I-squared value is high (51\%) for 3-24 month follow up.

4.4 Tratamiento quirúrgico en el glaucoma de ángulo abierto

4.4.1 Trabeculectomía en el tratamiento del glaucoma de ángulo abierto

4.4.1.1.Trabeculectomía respecto al tratamiento farmacológico

Question: Should initial medical treatment versus initial trabeculectomy be used for open angle glaucoma]?

Bibliography:

Burr J, Azuara-Blanco A, Avenell A. Medical versus surgical interventions for open angle glaucoma. Cochrane Database of Systematic Reviews 2004, Issue 2. Art. No.: CD004399. DOI: 10.1002/14651858.CD004399.pub2.

\begin{tabular}{|c|c|c|c|c|c|c|c|c|c|c|c|c|}
\hline \multicolumn{7}{|c|}{ Quality assessment } & \multicolumn{2}{|c|}{ No of patients } & \multicolumn{2}{|c|}{ Effect } & \multirow{2}{*}{ Quality } & \multirow{2}{*}{ Importance } \\
\hline $\begin{array}{l}\text { No of } \\
\text { studies }\end{array}$ & Design & $\begin{array}{l}\text { Risk of } \\
\text { bias }\end{array}$ & Inconsistency & Indirectness & Imprecision & $\begin{array}{c}\text { Other } \\
\text { considerations }\end{array}$ & $\begin{array}{c}\text { Initial } \\
\text { medical } \\
\text { treatment }\end{array}$ & $\begin{array}{c}\text { Initial } \\
\text { trabeculectom }\end{array}$ & $\begin{aligned} \text { Relative } \\
\text { y } \\
(95 \% \mathrm{Cl})\end{aligned}$ & Absolute & & \\
\hline \multicolumn{13}{|c|}{ Progressive visual field loss-medium-term (follow-up 1-5 years) } \\
\hline 1 & $\begin{array}{l}\text { randomise } \\
\text { trials }\end{array}$ & serious $^{1}$ & $\begin{array}{l}\text { no serious } \\
\text { inconsistency }\end{array}$ & $\begin{array}{l}\text { no serious } \\
\text { indirectness }\end{array}$ & serious $^{2}$ & none & $\begin{array}{c}27 / 57 \\
(47.4 \%)\end{array}$ & $\begin{array}{l}13 / 50 \\
(26 \%)\end{array}$ & $\begin{array}{c}\text { OR } \\
2.56 \\
(1.12 \\
\text { to } \\
5.83)\end{array}$ & $\begin{array}{c}214 \\
\text { more per } \\
1.000 \\
\text { (from } 22 \\
\text { more to } \\
412 \\
\text { more) }\end{array}$ & LOW & CRITICAL \\
\hline
\end{tabular}




\begin{tabular}{|c|c|c|c|c|c|c|c|c|c|c|c|}
\hline & essive visual field lo & loss-long-term & (5 years) (fol & llow-up >5; & asses & i: visua & Id scor & om basc & eline) & & \\
\hline 2 & \begin{tabular}{|l|} 
randomised \\
trials
\end{tabular} & $\begin{array}{l}3 \text { no serious } \\
\text { inconsistency }\end{array}$ & $\begin{array}{l}\text { no serious } \\
\text { indirectness }\end{array}$ & serious $^{2}$ & none & $\begin{array}{l}0 / 347 \\
(0 \%)\end{array}$ & $\begin{array}{l}0 / 348 \\
(0 \%)\end{array}$ & $\begin{array}{c}\text { not } \\
\text { pooled }\end{array}$ & $\begin{array}{c}\text { not } \\
\text { pooled }\end{array}$ & \begin{tabular}{ll|} 
पिएव \\
LOW
\end{tabular} & CRITICAL \\
\hline & change in IOP from & baseline-shor & rt-term (follo & w-up 1 year & rs; bett & ated bj & gher va & & & & \\
\hline 2 & $\begin{array}{l}\text { randomised } \\
\text { trials }\end{array}$ & $\begin{array}{l}\text { no serious } \\
\text { inconsistency }\end{array}$ & serious $^{4}$ & $\begin{array}{l}\text { no serious } \\
\text { imprecision }\end{array}$ & none & 87 & 103 & - & $\begin{array}{c}\text { mean } \\
6.14 \\
\text { higher } \\
(4.25 \text { to } \\
8.02 \\
\text { higher })\end{array}$ & \begin{tabular}{ll|} 
पिका \\
LOW
\end{tabular} & CRITICAL \\
\hline & change in IOP from & baseline-med & lium-term (fo & Dllow-up 1-5 & 5 years & indicat & y lowe & lies) & & & \\
\hline 1 & $\begin{array}{l}\text { randomised } \\
\text { trials }\end{array}$ & $\mid \begin{array}{l}\text { no serious } \\
\text { inconsistency }\end{array}$ & serious ${ }^{4}$ & serious $^{2}$ & none & 50 & 56 & - & $\begin{array}{c}\text { mean } \\
1.6 \\
\text { higher } \\
(0.69 \\
\text { lower to } \\
3.89 \\
\text { higher })\end{array}$ & $\begin{array}{l}\text { पिपर } \\
\text { VERY } \\
\text { LOW }\end{array}$ & CRITICAL \\
\hline & change in IOP from & baseline-long & -term (follow & $N$-up $>5$ yea & irs; bet & cated b & wer val & & & & \\
\hline 1 & \begin{tabular}{|l|}
$\mid \begin{array}{l}\text { randomised } \\
\text { trials }\end{array}$
\end{tabular} & $\begin{array}{l}1 \text { no serious } \\
\text { inconsistency }\end{array}$ & serious ${ }^{4}$ & serious $^{2}$ & none & 46 & 56 & - & $\begin{array}{c}\text { mean } \\
3.4 \\
\text { higher } \\
(1.04 \text { to } \\
5.76 \\
\text { higher })\end{array}$ & $\begin{array}{l}\text { पिमा } \\
\text { VERY } \\
\text { LOW }\end{array}$ & CRITICAL \\
\hline
\end{tabular}




\begin{tabular}{|c|c|c|c|c|c|c|c|c|c|c|c|}
\hline 1 & \begin{tabular}{|l|l} 
randomised & serious $^{3}$ \\
trials
\end{tabular} & $\begin{array}{l}\text { no serious } \\
\text { inconsistency }\end{array}$ & serious ${ }^{4}$ & \begin{tabular}{|l|} 
no serious \\
imprecision
\end{tabular} & none & 293 & 285 & - & \begin{tabular}{|c|} 
mean \\
2.2 \\
higher \\
$(1.63$ to \\
2.77 \\
higher)
\end{tabular} & $\begin{array}{ll}\text { पिवा } \\
\text { LOW }\end{array}$ & CRITICAL \\
\hline \multicolumn{12}{|c|}{ Visually significant cataract (follow-up 5 years) } \\
\hline 3 & \begin{tabular}{|l|l} 
randomised & serious 1 \\
trials
\end{tabular} & \begin{tabular}{|l} 
no serious \\
inconsistency
\end{tabular} & $\begin{array}{l}\text { no serious } \\
\text { indirectness }\end{array}$ & serious $^{2}$ & none & \begin{tabular}{|c|}
$57 / 403$ \\
$(14.1 \%)$
\end{tabular} & $\begin{array}{l}24 / 416 \\
(5.8 \%)\end{array}$ & $\begin{array}{c}\text { OR } \\
2.69 \\
(1.64 \\
\text { to } \\
4.42)\end{array}$ & \begin{tabular}{|c|}
84 more \\
per \\
1.000 \\
(from 34 \\
more to \\
155 \\
more)
\end{tabular} & \begin{tabular}{ll|} 
पिएव \\
LOW
\end{tabular} & CRITICAL \\
\hline
\end{tabular}

${ }^{1}$ Attrition bias/performance bias, detection bias and reporting bias unclear.

Small sample size/wide confidence interval/confidence intervals include benefit and harm.

CIGTS scores derive firld loos) were not reported for longer-term outcomes (five years or longer). They use a different index of visual field loss derived from the standard automated perimetry output. The study was thus judged at high risk of selective reporting bias.

Disease progression inerred from PIO.

${ }^{5}$ Analysis plot not shown and not heterogeneity test. 


\subsubsection{Trabeculectomía asociada a antimetabolitos en comparación a trabeculectomía sola}

Question: Intraoperative Mitomycin C versus control for glaucoma surgery [Data only. When citing this record quote "Cochrane Database of Systematic Reviews 2005, Issue 4".

\section{Bibliography:}

Wilkins M, Indar A, Wormald R. Intraoperative Mitomycin C for glaucoma surgery [Data only. When citing this record quote "Cochrane Database of Systematic Reviews 2005, Issue 4".]. Cochrane Database of Systematic Reviews [Year], Issue [Issue].

\begin{tabular}{|c|c|c|c|c|c|c|c|c|c|c|c|c|}
\hline \multicolumn{7}{|c|}{ Quality assessment } & \multicolumn{2}{|c|}{ No of patients } & \multicolumn{2}{|c|}{ Effect } & \multirow{2}{*}{ Quality } & \multirow{2}{*}{ Importance } \\
\hline \begin{tabular}{|l}
$\begin{array}{l}\text { No of } \\
\text { studies }\end{array}$ \\
\end{tabular} & Design & $\begin{array}{c}\text { Risk of } \\
\text { bias }\end{array}$ & Inconsistency & Indirectness & Imprecision & \begin{tabular}{|c|} 
Other \\
considerations
\end{tabular} & $\begin{array}{l}\text { Intraoperative } \\
\text { Mitomycin C }\end{array}$ & Control & $\begin{array}{l}\text { Relative } \\
(95 \% \mathrm{Cl})\end{array}$ & Absolute & & \\
\hline \multicolumn{13}{|c|}{ Mean intraocular pressure at 12 months (follow-up 1 years; better indicated by lower values) } \\
\hline 2 & \begin{tabular}{|l} 
randomised \\
trials
\end{tabular} & serious 1 & \begin{tabular}{|l} 
no serious \\
inconsistency
\end{tabular} & serious $^{2}$ & \begin{tabular}{|l} 
no serious \\
imprecision
\end{tabular} & none & 44 & 44 & - & $\begin{array}{c}\text { MD } 5.41 \\
\text { lower } \\
(7.34 \text { to } \\
3.49 \\
\text { lower })\end{array}$ & $\begin{array}{l}\text { Lिए। } \\
\text { LOW }\end{array}$ & CRITICAL \\
\hline \multicolumn{13}{|c|}{ Failure to control PIO at 12 months (follow-up 1 years) } \\
\hline 4 & $\begin{array}{l}\text { randomised } \\
\text { trials }\end{array}$ & serious 1 & \begin{tabular}{|l|} 
no serious \\
inconsistency
\end{tabular} & serious $^{2}$ & \begin{tabular}{|l} 
no serious \\
imprecision
\end{tabular} & none & $\begin{array}{l}18 / 231 \\
(7.8 \%)\end{array}$ & \begin{tabular}{|l|}
$30 / 107$ \\
$(28 \%)$
\end{tabular} & \begin{tabular}{|c|c}
$7 R$ \\
0.29 \\
$(0.16$ to \\
$0.53)$
\end{tabular} & $\begin{array}{c}199 \text { fewer } \\
\text { per } 1.000 \\
\text { (from } 132 \\
\text { fewer to } \\
236 \\
\text { fewer) }\end{array}$ & $\begin{array}{l}\square \square \square \\
\text { LOW }\end{array}$ & CRITICAL \\
\hline
\end{tabular}




\begin{tabular}{|c|c|c|c|c|c|c|c|c|c|c|c|}
\hline 2 & \begin{tabular}{l|l}
$\begin{array}{l}\text { randomised } \\
\text { trials }\end{array}$ &
\end{tabular} & $\begin{array}{l}\text { no serious } \\
\text { inconsistency }\end{array}$ & $\begin{array}{l}\text { no serious } \\
\text { indirectness }\end{array}$ & $\begin{array}{l}\text { very } \\
\text { serious }\end{array}$ & none & $\begin{array}{c}4 / 35 \\
(11.4 \%)\end{array}$ & $\begin{array}{c}1 / 22 \\
(4.5 \%)\end{array}$ & \begin{tabular}{|c|} 
OR \\
1.65 \\
$(0.16$ to \\
$17.47)$
\end{tabular} & \begin{tabular}{|c}
27 more \\
per 1.000 \\
(from 38 \\
fewer to \\
409 more)
\end{tabular} & $\begin{array}{l}\text { पिमा } \\
\text { VERY } \\
\text { LOW }\end{array}$ & CRITICAL \\
\hline \multicolumn{12}{|c|}{ Complications-hypotony } \\
\hline 3 & \begin{tabular}{|l|l|}
$\begin{array}{l}\text { randomised } \\
\text { trials }\end{array}$ & serious 1 \\
\end{tabular} & $\begin{array}{l}\text { no serious } \\
\text { inconsistency }\end{array}$ & $\begin{array}{l}\text { no serious } \\
\text { indirectness }\end{array}$ & serious $^{3}$ & none & $\begin{array}{l}4 / 65 \\
(6.2 \%)\end{array}$ & $\begin{array}{c}3 / 52 \\
(5.8 \%)\end{array}$ & \begin{tabular}{|c|} 
OR \\
1.05 \\
$(0.23$ to \\
$4.68)$
\end{tabular} & \begin{tabular}{|c|}
3 more \\
per 1.000 \\
(from 44 \\
fewer to \\
165 more)
\end{tabular} & $\begin{array}{l}\text { पिए। } \\
\text { LOW }\end{array}$ & CRITICAL \\
\hline \multicolumn{12}{|c|}{ Complications-cataract (follow-up 1 years) } \\
\hline 4 & \begin{tabular}{|l|l|} 
randomised \\
trials
\end{tabular} & $\begin{array}{l}\text { no serious } \\
\text { inconsistency }\end{array}$ & $\begin{array}{l}\text { no serious } \\
\text { indirectness }\end{array}$ & $\begin{array}{l}\text { very } \\
\text { serious }^{3}\end{array}$ & none & $\begin{array}{l}45 / 231 \\
(19.5 \%)\end{array}$ & $\begin{array}{l}10 / 107 \\
(9.3 \%)\end{array}$ & \begin{tabular}{|c|}
$\mathrm{RR}$ \\
1.93 \\
$(0.98$ to \\
$3.8)$
\end{tabular} & \begin{tabular}{|c|}
87 more \\
per 1.000 \\
(from 2 \\
fewer to \\
262 more)
\end{tabular} & $\begin{array}{l}\text { पिमा } \\
\text { VERY } \\
\text { LOW }\end{array}$ & CRITICAL \\
\hline
\end{tabular}

${ }^{1}$ Most of the studies are unclear about methodology.

${ }^{2}$ OAG progression as inferred from IOP.

${ }^{3}$ Wide confidence interval/confidence interval include benefit or appreciable harmsmall sample size/very low number of events. 
Question: Should intraoperative 5-FU vs placebo be used for glaucoma?

Bibliography:

Wong TT, Khaw PT, Aung T, Foster PJ, Htoon HM, Oen FTS, et al. The Singapore 5-Fluorouracil Trabeculectomy Study: Effects on Intraocular Pressure Control and Disease Progression at 3 Years. Ophthalmology. 1 de febrero de 2009; 116(2): 175-84.

\begin{tabular}{|c|c|c|c|c|c|c|c|c|c|c|c|c|}
\hline \multicolumn{7}{|c|}{ Quality assessment } & \multicolumn{2}{|c|}{ No of patients } & \multicolumn{2}{|c|}{ Effect } & \multirow{2}{*}{ Quality } & \multirow{2}{*}{ Importance } \\
\hline \begin{tabular}{|l} 
No of \\
studies
\end{tabular} & Design & $\begin{array}{c}\text { Risk of } \\
\text { bias }\end{array}$ & Inconsistency & Indirectness & Imprecision & \begin{tabular}{|c|}
$\begin{array}{c}\text { Other } \\
\text { considerations }\end{array}$ \\
\end{tabular} & \begin{tabular}{|c|} 
Intraoperative \\
$5-\mathrm{FU}$
\end{tabular} & Placebo & \begin{tabular}{|l|} 
Relative \\
$(95 \% \mathrm{Cl})$
\end{tabular} & Absolute & & \\
\hline \multicolumn{13}{|c|}{ Optic disc and visual field progression } \\
\hline 1 & $\begin{array}{l}\text { randomised } \\
\text { trials }\end{array}$ & serious ${ }^{1}$ & \begin{tabular}{|l|} 
no serious \\
inconsistency
\end{tabular} & \begin{tabular}{|l|} 
no serious \\
indirectness
\end{tabular} & serious $^{2}$ & none & $\begin{array}{c}14 / 111 \\
(12.6 \%)\end{array}$ & $\begin{array}{l}21 / 115 \\
(18.3 \%)\end{array}$ & \begin{tabular}{|c|} 
HR 0.67 \\
$(0.34$ to \\
$1.31)$
\end{tabular} & $\begin{array}{c}56 \text { fewer } \\
\text { per } 1.000 \\
\text { (from } 116 \\
\text { fewer to } \\
50 \text { more) }\end{array}$ & \begin{tabular}{|l|} 
पिएव \\
LOW
\end{tabular} & \\
\hline \multicolumn{13}{|c|}{ Intraocular pressure control failure } \\
\hline 1 & $\begin{array}{l}\text { randomised } \\
\text { trials }\end{array}$ & $\begin{array}{l}\text { no } \\
\text { serious } \\
\text { risk of } \\
\text { bias }\end{array}$ & \begin{tabular}{|l|} 
no serious \\
inconsistency
\end{tabular} & serious $^{3}$ & serious $^{2}$ & none & $\begin{array}{l}32 / 110 \\
(29.1 \%)\end{array}$ & \begin{tabular}{|l|}
$42 / 118$ \\
$(35.6 \%)$
\end{tabular} & \begin{tabular}{|c|}
$R R$ \\
0.854 \\
$(0.63$ to \\
$1.16)$
\end{tabular} & $\begin{array}{c}52 \text { fewer } \\
\text { per } 1.000 \\
\text { (from } 132 \\
\text { fewer to } \\
57 \text { more) }\end{array}$ & \begin{tabular}{|l|} 
पिएव \\
LOW
\end{tabular} & CRITICAL \\
\hline
\end{tabular}

1 No ITT.
2 The confidence interval crosses the threshold/includes appreciable benefit or harm.

${ }_{3}$ OAG progression as inferred from IOP. 
Question: Regular dose postoperative 5-FU versus control for glaucoma surgery

Bibliography:

Wormald R, Wilkins M, Bunce C. Postoperative 5-Fluorouracil for glaucoma surgery [Data only. When citing this record quote "Cochrane Database of Systematic Reviews 2001, Issue 3".]. Cochrane Database of Systematic Reviews [Year], Issue [Issue].

\begin{tabular}{|c|c|c|c|c|c|c|c|c|c|c|c|c|}
\hline \multicolumn{7}{|c|}{ Quality assessment } & \multicolumn{2}{|c|}{ No of patients } & \multicolumn{2}{|c|}{ Effect } & \multirow{2}{*}{ Quality } & \multirow{2}{*}{ Importance } \\
\hline $\begin{array}{l}\text { No of } \\
\text { studies }\end{array}$ & Design & $\begin{array}{c}\text { Risk of } \\
\text { bias }\end{array}$ & Inconsistency & Indirectness & Imprecision & $\begin{array}{c}\text { Other } \\
\text { considerations }\end{array}$ & \begin{tabular}{|c} 
Regular dose \\
postoperative \\
5-FU
\end{tabular} & Control & $\begin{array}{l}\text { Relative } \\
\text { (95\% Cl) }\end{array}$ & Absolute & & \\
\hline \multicolumn{13}{|c|}{ Mean intraocular pressure at 12 months-primary trabeculectomy (follow-up 0-12 months; better indicated by lower values) } \\
\hline 2 & $\begin{array}{l}\text { randomisec } \\
\text { trials }\end{array}$ & serious $^{1}$ & $\begin{array}{l}\text { no serious } \\
\text { inconsistency }\end{array}$ & serious $^{2}$ & $\begin{array}{l}\text { no serious } \\
\text { imprecision }\end{array}$ & none & 57 & 55 & - & \begin{tabular}{|c|} 
MD 4.67 \\
lower $(6.6$ \\
to 2.74 \\
lower)
\end{tabular} & $\begin{array}{l}\text { LOपए } \\
\text { LOW }\end{array}$ & CRITICAL \\
\hline \multicolumn{13}{|c|}{ Failure at 12 months-primary trabeculectomy (follow-up 0-12 months) } \\
\hline 2 & $\begin{array}{l}\text { randomise } \\
\text { trials }\end{array}$ & serious ${ }^{1}$ & $\begin{array}{l}\text { no serious } \\
\text { inconsistency }\end{array}$ & serious $^{3}$ & $\begin{array}{l}\text { very } \\
\text { serious }{ }^{4}\end{array}$ & none & $\begin{array}{c}3 / 57 \\
(5.3 \%)\end{array}$ & $\begin{array}{c}14 / 55 \\
(25.5 \%)\end{array}$ & $\begin{array}{c}\mathrm{RR} \\
0.21 \\
(0.06 \text { to } \\
0.68)\end{array}$ & $\begin{array}{c}201 \text { fewer } \\
\text { per } 1.000 \\
\text { (from } 81 \\
\text { fewer to } \\
239 \\
\text { fewer) }\end{array}$ & \begin{tabular}{|l|} 
VID० \\
VERY \\
LOW
\end{tabular} & CRITICAL \\
\hline \multicolumn{13}{|c|}{ Complications-wound leak-primary trabeculectomy } \\
\hline 2 & $\begin{array}{l}\text { randomise } \\
\text { trials }\end{array}$ & serious ${ }^{1}$ & $\begin{array}{l}\text { no serious } \\
\text { inconsistency }\end{array}$ & \begin{tabular}{|l} 
no serious \\
indirectness
\end{tabular} & $\begin{array}{l}\text { very } \\
\text { serious }\end{array}$ & none & $\begin{array}{c}1 / 57 \\
(1.8 \%)\end{array}$ & \begin{tabular}{|c|}
$2 / 55$ \\
$(3.6 \%)$
\end{tabular} & $\begin{array}{c}\mathrm{RR} \\
0.47 \\
(0.04 \text { to }\end{array}$ & $\begin{array}{c}19 \text { fewer } \\
\text { per } 1.000 \\
\text { (from } 35\end{array}$ & \begin{tabular}{|l|l|} 
पि口प \\
VERY
\end{tabular} & \\
\hline
\end{tabular}




\begin{tabular}{|l|l|l|l|l|l|l|l|l|l|l|l|}
\hline & & & & & & $4.91)$ & $\begin{array}{c}\text { fewer to } \\
142 \\
\text { more })\end{array}$ & $\begin{array}{l}\text { LOW } \\
\text { Complications-hypotonous maculopathy }\end{array}$ \\
\hline 1 & $\begin{array}{l}\text { randomised } \\
\text { trials }\end{array}$ \\
\hline
\end{tabular}

${ }^{1}$ All risk of bias are unclear.

2 OAG progression as inferred from IOP.

No explanation was provided.

Wide confidence interval.

${ }^{5}$ Only one study have events.

${ }^{6}$ Small sample size/low number of events/confidence interval include appreciable benefit and appreciable harm. 
Question: Low dose postoperative 5-FU versus control for glaucoma surgery [Data only. When citing this record quote "Cochrane Database of Systematic Reviews 2001, Issue 3".]

\section{Bibliography:}

Wormald R, Wilkins M, Bunce C. Postoperative 5-Fluorouracil for glaucoma surgery [Data only. When citing this record quote "Cochrane Database of Systematic Reviews 2001, Issue 3".]. Cochrane Database of Systematic Reviews [Year], Issue [Issue].

\begin{tabular}{|c|c|c|c|c|c|c|c|c|c|c|c|c|}
\hline \multicolumn{7}{|c|}{ Quality assessment } & \multicolumn{2}{|c|}{ No of patients } & \multicolumn{2}{|c|}{ Effect } & \multirow{2}{*}{ Quality } & \multirow{2}{*}{ Importance } \\
\hline $\begin{array}{c}\text { No of } \\
\text { studies }\end{array}$ & Design & $\begin{array}{c}\text { Risk of } \\
\text { bias }\end{array}$ & Inconsistency & Indirectness & Imprecision & n $\begin{array}{c}\text { Other } \\
\text { considerations }\end{array}$ & \begin{tabular}{|c|} 
Low dose \\
postoperative \\
$5-\mathrm{FU}$
\end{tabular} & Control & $\begin{array}{l}\text { Relative } \\
(95 \% \mathrm{Cl})\end{array}$ & Absolute & & \\
\hline \multicolumn{13}{|c|}{ Mean intraocular pressure at 12 months (better indicated by lower values) } \\
\hline 1 & $\begin{array}{l}\text { randomisec } \\
\text { trials }\end{array}$ & serious $^{1}$ & $\begin{array}{l}\text { no serious } \\
\text { inconsistency }\end{array}$ & serious $^{2}$ & serious $^{3}$ & none & 38 & 38 & - & $\begin{array}{c}\text { MD } 0.5 \\
\text { lower } \\
(2.96 \\
\text { lower to } \\
1.96 \\
\text { higher) }\end{array}$ & \begin{tabular}{|l|} 
पिएव \\
VERY \\
LOW
\end{tabular} & CRITICAL \\
\hline \multicolumn{13}{|c|}{ Failure at 12 months (follow-up 0-12 months) } \\
\hline 1 & $\begin{array}{l}\text { randomisec } \\
\text { trials }\end{array}$ & serious $^{1}$ & $\begin{array}{l}\text { no serious } \\
\text { inconsistency }\end{array}$ & serious $^{2}$ & serious $^{3}$ & none & $\begin{array}{c}26 / 38 \\
(68.4 \%)\end{array}$ & \begin{tabular}{|c|}
$28 / 38$ \\
$(73.7 \%)$
\end{tabular} & \begin{tabular}{|c} 
RR 0.93 \\
$(0.7$ to \\
$1.24)$
\end{tabular} & $\begin{array}{c}52 \text { fewer } \\
\text { per } 1.000 \\
\text { (from } 221 \\
\text { fewer to } \\
177 \text { more) }\end{array}$ & $\begin{array}{l}\text { VERT } \\
\text { LOW } \\
\end{array}$ & CRITICAL \\
\hline \multicolumn{13}{|c|}{ Complications-wound leak } \\
\hline 2 & $\begin{array}{l}\text { randomisec } \\
\text { trials }\end{array}$ & serious ${ }^{1}$ & serious ${ }^{4}$ & \begin{tabular}{|l|} 
no serious \\
indirectness
\end{tabular} & serious $^{3}$ & none & $\begin{array}{c}9 / 61 \\
(14.8 \%)\end{array}$ & \begin{tabular}{|c|}
$4 / 58$ \\
$(6.9 \%)$
\end{tabular} & \begin{tabular}{|l|} 
RR 1.88 \\
$(0.68$ to
\end{tabular} & $\begin{array}{c}61 \text { more } \\
\text { per } 1.000\end{array}$ & \begin{tabular}{|l|} 
पि口प \\
VERY
\end{tabular} & \\
\hline
\end{tabular}




\begin{tabular}{|c|c|c|c|c|c|c|c|c|c|c|c|}
\hline & & & & & & & & & $5.24)$ & \begin{tabular}{|c|} 
(from 22 \\
fewer to \\
292 more)
\end{tabular} & LOW \\
\hline \multicolumn{12}{|c|}{ Complications-hypotonous maculopathy } \\
\hline 1 & $\begin{array}{l}\text { randomised } \\
\text { trials }\end{array}$ & serious ${ }^{1}$ & $\begin{array}{l}1 \text { no serious } \\
\text { inconsistency }\end{array}$ & $\begin{array}{l}\text { no serious } \\
\text { indirectness }\end{array}$ & $\begin{array}{l}\text { very } \\
\text { serious }\end{array}$ & none & $\begin{array}{c}4 / 23 \\
(17.4 \%)\end{array}$ & $\begin{array}{l}0 / 20 \\
(0 \%)\end{array}$ & $\begin{array}{l}\text { RR } 7.88 \\
(0.45 \text { to } \\
137.85)\end{array}$ & - & $\begin{array}{l}\text { पिए। } \\
\text { VERY } \\
\text { LOW }\end{array}$ \\
\hline \multicolumn{12}{|c|}{ Complications-cataract } \\
\hline 1 & $\begin{array}{l}\text { randomised } \\
\text { trials }\end{array}$ & serious 1 & $\begin{array}{l}1 \text { no serious } \\
\text { inconsistency }\end{array}$ & $\begin{array}{l}\text { no serious } \\
\text { indirectness }\end{array}$ & $\begin{array}{l}\text { very } \\
\text { serious }^{3}\end{array}$ & none & $\begin{array}{c}6 / 38 \\
(15.8 \%)\end{array}$ & $\begin{array}{c}1 / 38 \\
(2.6 \%)\end{array}$ & $\begin{array}{c}\text { not } \\
\text { pooled }\end{array}$ & not pooled & 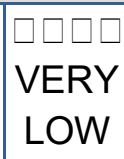 \\
\hline
\end{tabular}

${ }^{1}$ All risk of bias are unclear.

OAG progression as inferred from IOP

Wide confidence intervals/Small sample size.

Effect confidence intervals partially overlap. One study suggest benefit and the other one harm. 
4.4.2. Cirugía no penetrante en el tratamiento del glaucoma de ángulo abierto 4.4.2.1. Cirugía no penetrante respecto a la trabeculectomía

Question: Should non-penetrating trabecular surgery vs trabeculectomy be used for open-angle glaucoma? Bibliography:

Ke M, Guo J, Qian Z. Meta analysis of non-penetrating trabecular surgery versus trabeculectomy for the treatment of open angle glaucoma. J Huazhong Univ Sci Technolog Med Sci. 2011; 31(2): 264-70.

\begin{tabular}{|c|c|c|c|c|c|c|c|c|c|c|c|c|}
\hline \multicolumn{7}{|c|}{ Quality assessment } & \multicolumn{2}{|c|}{ No of patients } & \multicolumn{2}{|r|}{ Effect } & \multirow{2}{*}{ Quality } & \multirow{2}{*}{ I Importance } \\
\hline $\begin{array}{l}\begin{array}{l}\text { No of } \\
\text { studies }\end{array} \\
\text { stion }\end{array}$ & Design & $\begin{array}{c}\text { Risk of } \\
\text { bias }\end{array}$ & Inconsistency & Indirectness & s Imprecision & $\begin{array}{c}\text { Other } \\
\text { considerations }\end{array}$ & \begin{tabular}{|c|} 
Non- \\
penetrating \\
trabecular \\
surgery
\end{tabular} & Trabeculectomy & $\begin{array}{c}\text { Relative } \\
(95 \% \\
\mathrm{Cl})\end{array}$ & Absolute & & \\
\hline \multicolumn{13}{|c|}{ IOP reduction (follow-up mean 12 months; better indicated by lower values) } \\
\hline 6 & $\begin{array}{l}\text { randomise } \\
\text { trials }\end{array}$ & serious 1 & $\begin{array}{l}\text { no serious } \\
\text { inconsistency }\end{array}$ & serious $^{2}$ & $\begin{array}{l}\text { no serious } \\
\text { imprecision }\end{array}$ & none ${ }^{3}$ & 158 & 158 & - & \begin{tabular}{|c|} 
MD 2.83 \\
higher (1.91 \\
to 3.74 \\
higher)
\end{tabular} & $\begin{array}{l}\text { पिए। } \\
\text { LOW }\end{array}$ & CRITICAL \\
\hline \multicolumn{13}{|c|}{ Success rate } \\
\hline 6 & $\begin{array}{l}\text { randomise } \\
\text { trials }\end{array}$ & serious 1 & $\begin{array}{l}\text { no serious } \\
\text { inconsistency }\end{array}$ & serious $^{2}$ & $\begin{array}{l}\text { no serious } \\
\text { imprecision }\end{array}$ & none ${ }^{3}$ & $\begin{array}{l}90 / 158 \\
(57 \%)\end{array}$ & $\begin{array}{l}119 / 158 \\
(75.3 \%)\end{array}$ & \begin{tabular}{|c|}
$\mathrm{RR}$ \\
0.34 \\
$(0.2$ to \\
$0.59)$
\end{tabular} & \begin{tabular}{|c|}
497 fewer \\
per 1.000 \\
(from 309 \\
fewer to \\
603 fewer)
\end{tabular} & $\begin{array}{l}\text { पिए। } \\
\text { LOW }\end{array}$ & CRITICAL \\
\hline
\end{tabular}

${ }^{1}$ None of the studies detailed randomization and concealment allocation methods, only one detailed study withdrawals. 
${ }^{2}$ There were differences in follow-up, outcomes definition and characteristics. IOP considered as a surrogate outcome of visual field loss in patient with open angle glaucoma.

Publication bias not detected. The number of papers and sample sizes covered by the study was limited and the results of unpublished studies are not clear.

Question: Should deep sclerectomy vs trabeculectomy be used for open-angle glaucoma?

Bibliography:

Cheng JW, Xi GL, Wei RL, Cai JP, Li Y. Efficacy and tolerability of nonpenetrating filtering surgery in the treatment of open-angle glaucoma: a meta-analysis. Ophthalmologica. 2010;224(3): 138-46.

Cheng JW, Xi GL, Wei RL, Cai JP, Li Y. Efficacy and tolerability of nonpenetrating glaucoma surgery augmented with mitomycin C in treatment of open-angle glaucoma: a meta-analysis. Can J Ophthalmol. 2009 Feb;44(1): 76-82.

Ke M, Guo J, Qian Z. Meta analysis of non-penetrating trabecular surgery versus trabeculectomy for the treatment of open angle glaucoma J Huazhong Univ Sci Technolog Med Sci. 2011 Apr; 31(2): 264-70.

\begin{tabular}{|c|c|c|c|c|c|c|c|c|c|c|c|c|}
\hline \multicolumn{7}{|c|}{ Quality assessment } & \multicolumn{2}{|c|}{ No of patients } & \multicolumn{2}{|c|}{ Effect } & \multirow{2}{*}{ Quality } & \multirow{2}{*}{ Importance } \\
\hline \begin{tabular}{|} 
No of \\
studies
\end{tabular} & Design & $\begin{array}{c}\text { Risk of } \\
\text { bias }\end{array}$ & Inconsistency & Indirectness & Imprecision & $\begin{array}{c}\text { Other } \\
\text { considerations }\end{array}$ & $\begin{array}{c}\text { Deep } \\
\text { sclerectomy }\end{array}$ & Trabeculectomy & \begin{tabular}{|c} 
Relative \\
$(95 \%$ \\
Cl)
\end{tabular} & Absolute & & \\
\hline \multicolumn{13}{|c|}{ Success rate (follow-up median 12 months) } \\
\hline 5 & $\begin{array}{l}\text { randomise } \\
\text { trials }\end{array}$ & $\begin{array}{l}\mathrm{d} \text { no } \\
\text { serious } \\
\text { risk of } \\
\text { bias }\end{array}$ & $\begin{array}{l}\text { no serious } \\
\text { inconsistency }\end{array}$ & serious ${ }^{1}$ & serious $^{2}$ & none & $\begin{array}{c}83 / 132 \\
(62.9 \%)\end{array}$ & $\begin{array}{l}97 / 130 \\
(74.6 \%)\end{array}$ & $\begin{array}{c}\mathrm{RR} \\
0.90 \\
(0.79 \\
\text { to } \\
1.02)\end{array}$ & \begin{tabular}{|c|}
75 \\
fewer \\
per \\
1.000 \\
(from \\
157 \\
fewer \\
to 15 \\
more)
\end{tabular} & $\begin{array}{l}\square \square \square \\
\text { LOW }\end{array}$ & CRITICAL \\
\hline
\end{tabular}




\begin{tabular}{|c|c|c|c|c|c|c|c|c|c|c|c|c|}
\hline \multicolumn{13}{|c|}{ Posoperative adverse event: hifema } \\
\hline 7 & $\begin{array}{l}\text { randomised } \\
\text { trials }\end{array}$ & \begin{tabular}{|l|} 
no \\
serious \\
risk of \\
bias
\end{tabular} & $\begin{array}{l}\text { no serious } \\
\text { inconsistency }\end{array}$ & \begin{tabular}{|c|} 
no serious \\
y indirectness
\end{tabular} & serious ${ }^{2}$ & none & $\begin{array}{l}12 / 270 \\
(4.4 \%)\end{array}$ & $\begin{array}{c}35 / 243 \\
(14.4 \%)\end{array}$ & \begin{tabular}{|c|}
$R R$ \\
0.89 \\
$(0.8$ to \\
$0.98)$
\end{tabular} & $\begin{array}{c}16 \\
\text { fewer } \\
\text { per } \\
1.000 \\
\text { (from } 3 \\
\text { fewer } \\
\text { to } 29 \\
\text { fewer) }\end{array}$ & MODERATE & $=$ \\
\hline \multicolumn{13}{|c|}{ Posoperative adverse event: hypotony } \\
\hline 6 & $\begin{array}{l}\text { randomised } \\
\text { trials }\end{array}$ & \begin{tabular}{|l|}
$\mid$ no \\
serious \\
risk of \\
bias
\end{tabular} & $\begin{array}{l}\text { no serious } \\
\text { inconsistency }\end{array}$ & \begin{tabular}{|l|} 
no serious \\
y indirectness
\end{tabular} & serious ${ }^{2}$ & none & $\begin{array}{c}9 / 284 \\
(3.2 \%)\end{array}$ & $\begin{array}{c}28 / 260 \\
(10.8 \%)\end{array}$ & $\begin{array}{c}\mathrm{RR} \\
0.91 \\
(0.84 \\
\text { to } \\
0.99)\end{array}$ & $\begin{array}{c}10 \\
\text { fewer } \\
\text { per } \\
1.000 \\
\text { (from } 1 \\
\text { fewer } \\
\text { to } 17 \\
\text { fewer) }\end{array}$ & 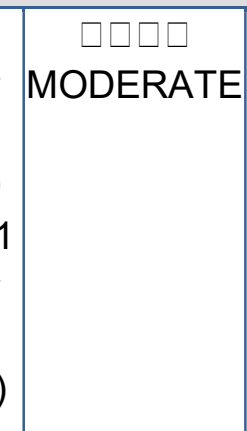 & CRITICAL \\
\hline \multicolumn{13}{|c|}{ Posoperative adverse event: choroidal detachment } \\
\hline 4 & $\begin{array}{l}\text { randomised } \\
\text { trials }\end{array}$ & \begin{tabular}{|l|} 
no \\
serious \\
risk of \\
bias
\end{tabular} & $\begin{array}{l}\text { no serious } \\
\text { inconsistency }\end{array}$ & $\begin{array}{l}\text { no serious } \\
\text { yndirectness }\end{array}$ & serious ${ }^{2}$ & none & $\begin{array}{c}71 / 190 \\
(37.4 \%)\end{array}$ & $\begin{array}{c}83 / 165 \\
(50.3 \%)\end{array}$ & $\begin{array}{c}\mathrm{RR} \\
0.84 \\
(0.75 \\
\text { to } \\
0.93)\end{array}$ & $\begin{array}{c}80 \\
\text { fewer } \\
\text { per } \\
1.000 \\
\text { (from } \\
35 \\
\text { fewer } \\
\text { to } 126 \\
\text { fewer) }\end{array}$ & MODERATE & $\equiv$ CRITICAL \\
\hline
\end{tabular}




\begin{tabular}{|c|c|c|c|c|c|c|c|c|c|c|c|c|}
\hline 4 & $\begin{array}{l}\text { randomised } \\
\text { trials }\end{array}$ & \begin{tabular}{|l|} 
no \\
serious \\
risk of \\
bias
\end{tabular} & $\begin{array}{l}\text { no serious } \\
\text { inconsistency }\end{array}$ & no serious & serious ${ }^{2}$ & none & $\begin{array}{l}11 / 152 \\
(7.2 \%)\end{array}$ & $\begin{array}{l}47 / 150 \\
(31.3 \%)\end{array}$ & \begin{tabular}{|c}
$\mathrm{RR}$ \\
0.77 \\
$(0.5$ to \\
$0.96)$
\end{tabular} & \begin{tabular}{|c}
72 \\
fewer \\
per \\
1.000 \\
(from \\
13 \\
fewer \\
to 157 \\
fewer)
\end{tabular} & 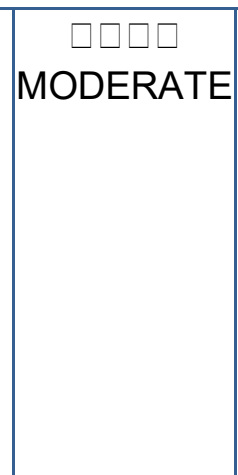 & CRITICAL \\
\hline
\end{tabular}

IIOP considered as a surrogate outcome of visual field loss in patient with open angle glaucoma. The analyses of rates of success and postoperative complications were based on data pooled from trials with different follow-up periods. Another potencial source of heterogegeneity in the results is the assessment criteria of succes.

${ }^{2}$ Small sample size, few events in the treatment and control groups. 
Question: Should comparison of viscocanalostomy vs trabeculectomy be used for open-angle glaucoma? Bibliography:

Cheng JW, Xi GL, Wei RL, Cai JP, Li Y. Efficacy and tolerability of nonpenetrating filtering surgery in the treatment of open-angle glaucoma: a meta-analysis. Ophthalmologica. 2010;224(3): 138-46.

Chai C, Loon SC. Meta-analysis of viscocanalostomy versus trabeculectomy in uncontrolled glaucoma. J Glaucoma. 2010;19(8): 519-27.

\begin{tabular}{|c|c|c|c|c|c|c|c|c|c|c|c|c|}
\hline \multicolumn{7}{|c|}{ Quality assessment } & \multicolumn{2}{|c|}{ No of patients } & \multicolumn{2}{|c|}{ Effect } & \multirow{2}{*}{ Quality } & \multirow{2}{*}{ Importance } \\
\hline $\begin{array}{l}\text { No of } \\
\text { studies }\end{array}$ & Design & $\begin{array}{c}\text { Risk of } \\
\text { bias }\end{array}$ & Inconsistency & Indirectness & Imprecision & $\begin{array}{c}\text { Other } \\
\text { considerations }\end{array}$ & $\begin{array}{c}\text { Comparison of } \\
\text { viscocanalostomy }\end{array}$ & Trabeculectomy & $\begin{array}{c}\text { Relative } \\
(95 \% \\
\text { Cl) }\end{array}$ & Absolute & & \\
\hline \multicolumn{13}{|c|}{ Reducción de la PIO a los 6 meses (better indicated by lower values) } \\
\hline 8 & $\begin{array}{l}\text { randomisec } \\
\text { trials }\end{array}$ & $\begin{array}{l}\text { no } \\
\text { serious } \\
\text { risk of } \\
\text { bias }\end{array}$ & $\mid \begin{array}{l}\text { no serious } \\
\text { inconsistency }\end{array}$ & $\begin{array}{l}\text { very } \\
\text { serious }{ }^{1}\end{array}$ & $\begin{array}{l}\text { no serious } \\
\text { imprecision }\end{array}$ & none & 168 & 169 & - & \begin{tabular}{|c|} 
MD \\
2.25 \\
higher \\
$(1.38$ to \\
3.12 \\
higher)
\end{tabular} & $\begin{array}{l}\text { पिएव } \\
\text { LOW }\end{array}$ & CRITICAL \\
\hline \multicolumn{13}{|c|}{ Reducción de la PIO a los 12 meses (better indicated by lower values) } \\
\hline 6 & $\begin{array}{l}\text { randomisec } \\
\text { trials }\end{array}$ & $\begin{array}{l}\text { no } \\
\text { serious } \\
\text { risk of } \\
\text { bias }\end{array}$ & $\begin{array}{l}\text { no serious } \\
\text { inconsistency }\end{array}$ & $\begin{array}{l}\text { very } \\
\text { serious }{ }^{1}\end{array}$ & $\begin{array}{l}\text { no serious } \\
\text { imprecision }\end{array}$ & none & 148 & 141 & - & \begin{tabular}{|c|}
$\mathrm{MD}$ \\
3.64 \\
higher \\
$(2.75$ to \\
4.54 \\
higher)
\end{tabular} & $\begin{array}{l}\text { पिएव } \\
\text { LOW }\end{array}$ & CRITICAL \\
\hline dt & n & $\mathbf{O a}$ & ese & tter ind & ed by lowe & ver values) & & & & & & \\
\hline
\end{tabular}




\begin{tabular}{|c|c|c|c|c|c|c|c|c|c|c|c|c|}
\hline 3 & $\begin{array}{l}\text { randomised } \\
\text { trials }\end{array}$ & \begin{tabular}{|l|} 
no \\
serious \\
risk of \\
bias
\end{tabular} & $\begin{array}{l}\text { no serious } \\
\text { inconsistency }\end{array}$ & $\begin{array}{l}\text { very } \\
\text { y serious }{ }^{1}\end{array}$ & \begin{tabular}{|l|} 
no serious \\
imprecision
\end{tabular} & none & 67 & 66 & - & \begin{tabular}{|c|}
$\mathrm{MD}$ \\
3.42 \\
higher \\
$(1.8$ to \\
5.03 \\
higher $)$
\end{tabular} & \begin{tabular}{l|} 
पिए। \\
LOW
\end{tabular} & CRITICAL \\
\hline \multicolumn{13}{|c|}{ Success rate } \\
\hline 3 & $\begin{array}{l}\text { randomised } \\
\text { trials }\end{array}$ & $\begin{array}{l}\text { no } \\
\text { serious } \\
\text { risk of } \\
\text { bias }\end{array}$ & \begin{tabular}{|l|} 
no serious \\
inconsistency
\end{tabular} & y serious $^{1}$ & serious $^{2}$ & none & $\begin{array}{c}37 / 80 \\
(46.3 \%)\end{array}$ & $\begin{array}{c}51 / 80 \\
(63.8 \%)\end{array}$ & \begin{tabular}{|c|}
$\mathrm{RD}-$ \\
$0.16(0$ \\
to 0$)$
\end{tabular} & \begin{tabular}{|c|}
739 \\
fewer \\
per \\
1.000 \\
(from \\
637 \\
fewer \\
to 637 \\
fewer)
\end{tabular} & $\begin{array}{l}\text { LOW } \\
\text { LOW }\end{array}$ & CRITICAL \\
\hline \multicolumn{13}{|c|}{ Posoperative adverse event: cataract } \\
\hline 5 & $\begin{array}{l}\text { randomised } \\
\text { trials }\end{array}$ & \begin{tabular}{|l|} 
no \\
serious \\
risk of \\
bias
\end{tabular} & \begin{tabular}{|l|} 
no serious \\
inconsistency
\end{tabular} & \begin{tabular}{|l|} 
no serious \\
yindirectness
\end{tabular} & serious ${ }^{2}$ & none & $\begin{array}{l}7 / 201 \\
(3.5 \%)\end{array}$ & $\begin{array}{c}26 / 202 \\
(12.9 \%)\end{array}$ & \begin{tabular}{c|}
$\mathrm{RR}$ \\
0.31 \\
$(0.15$ \\
to \\
$0.64)$
\end{tabular} & \begin{tabular}{|c}
89 \\
fewer \\
per \\
1.000 \\
(from \\
46 \\
fewer \\
to 109 \\
fewer)
\end{tabular} & \begin{tabular}{c|c|} 
MODI \\
MODERATE
\end{tabular} & CRITICAL \\
\hline \multicolumn{13}{|c|}{ Posoperative adverse event: hypotony } \\
\hline 9 & \begin{tabular}{|l|} 
randomised \\
trials
\end{tabular} & $\mid \begin{array}{l}\text { no } \\
\text { serious }\end{array}$ & \begin{tabular}{|l|} 
no serious \\
inconsistency
\end{tabular} & $\mid \begin{array}{l}\text { no serious } \\
\text { indirectness }\end{array}$ & serious ${ }^{2}$ & none & $\begin{array}{c}8 / 211 \\
(3.8 \%)\end{array}$ & $\begin{array}{c}30 / 212 \\
(14.2 \%)\end{array}$ & \begin{tabular}{c|} 
RR \\
0.29
\end{tabular} & \begin{tabular}{|c|}
100 \\
fewer
\end{tabular} & $\begin{array}{c}\square 00 \square \\
\text { MODERATE }\end{array}$ & CRITICAL \\
\hline
\end{tabular}




\begin{tabular}{|c|c|c|c|c|c|c|c|c|c|c|c|c|}
\hline & & $\begin{array}{l}\text { risk of } \\
\text { bias }\end{array}$ & & & & & & & $\begin{array}{l}(0.15 \\
\text { to } \\
0.58)\end{array}$ & $\begin{array}{c}\text { per } \\
1.000 \\
\text { (from } \\
59 \\
\text { fewer } \\
\text { to } 120 \\
\text { fewer) }\end{array}$ & & \\
\hline \multicolumn{13}{|c|}{ Posoperative adverse event: hyphema } \\
\hline 9 & \begin{tabular}{l|l|} 
randomised & r \\
trials & s \\
& $b$
\end{tabular} & \begin{tabular}{|l|} 
no \\
serious \\
risk of \\
bias
\end{tabular} & $\begin{array}{l}\text { no serious } \\
\text { inconsistency }\end{array}$ & \begin{tabular}{|l|} 
no serious \\
yndirectness
\end{tabular} & serious ${ }^{2}$ & none & $\begin{array}{l}17 / 211 \\
(8.1 \%)\end{array}$ & $\begin{array}{c}35 / 212 \\
(16.5 \%)\end{array}$ & \begin{tabular}{|c}
$\mathrm{RR}$ \\
0.50 \\
$(0.3$ to \\
$0.84)$
\end{tabular} & $\begin{array}{c}83 \\
\text { fewer } \\
\text { per } \\
1.000 \\
\text { (from } \\
26 \\
\text { fewer } \\
\text { to } 116 \\
\text { fewer) }\end{array}$ & $\begin{array}{c}\text { पIDI } \\
\text { MODERATE }\end{array}$ & CRITICAL \\
\hline \multicolumn{13}{|c|}{ Posoperative adverse event: shallow anterior chamber } \\
\hline 9 & \begin{tabular}{|l|l|} 
randomised & r \\
trials & s \\
& $b$
\end{tabular} & \begin{tabular}{|l|} 
no \\
serious \\
risk of \\
bias
\end{tabular} & $\begin{array}{l}\text { no serious } \\
\text { inconsistency }\end{array}$ & \begin{tabular}{|l|} 
no serious \\
indirectness
\end{tabular} & serious $^{2}$ & none & $\begin{array}{l}11 / 167 \\
(6.6 \%)\end{array}$ & $\begin{array}{l}33 / 165 \\
(20 \%)\end{array}$ & \begin{tabular}{|c|}
$\mathrm{RR}$ \\
0.19 \\
$(0.08$ \\
to \\
$0.45)$
\end{tabular} & $\begin{array}{c}162 \\
\text { fewer } \\
\text { per } \\
1.000 \\
\text { (from } \\
110 \\
\text { fewer } \\
\text { to } 184 \\
\text { fewer) }\end{array}$ & $\begin{array}{c}\text { प्रा } \\
\text { MODERATE }\end{array}$ & CRITICAL \\
\hline \multicolumn{13}{|c|}{ Posoperative adverse event: perforation of descemet membrane } \\
\hline 6 & randomised $n$ & & no serious & no serious & serious $^{2}$ & none & $21 / 159$ & $0 / 152$ & RR & 44 & 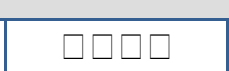 & CRITICAL \\
\hline
\end{tabular}




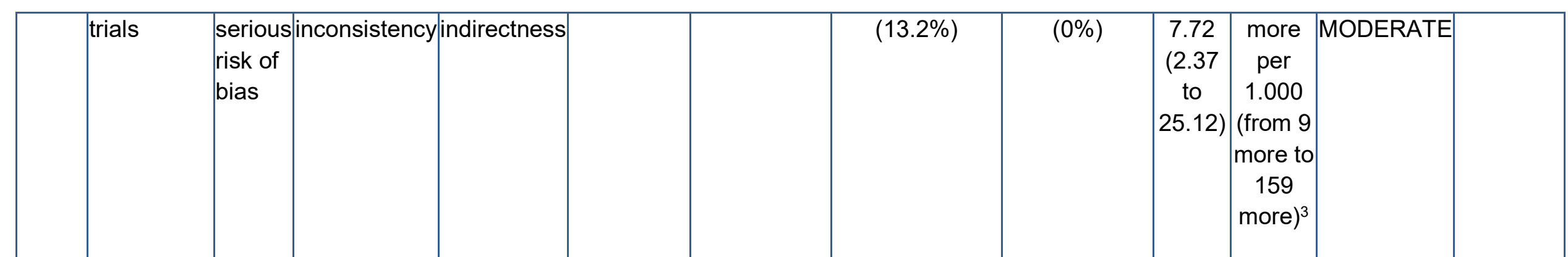

IOP considered as a surrogate outcome of visual field loss in patient with open angle glaucoma. Follow-up period was different between ECA. The IOP normal values were different between the studies.

2 There were few events in both groups (intervention and control).

${ }^{3}$ Calculated from 1 event in the control group. 
Question: Should nonpenetrating glaucoma surgery plus mitomycin C vs trabeculectomy plus mitomycin C be used for open-angle glaucoma?

\section{Bibliography:}

Cheng JW, Xi GL, Wei RL, Cai JP, Li Y. Efficacy and tolerability of nonpenetrating glaucoma surgery augmented with mitomycin C in treatment of open-angle glaucoma: a meta-analysis. Can J Ophthalmol. 2009; 44(1): 76-82.

\begin{tabular}{|c|c|c|c|c|c|c|c|c|c|c|c|c|}
\hline \multicolumn{7}{|c|}{ Quality assessment } & \multicolumn{2}{|c|}{ No of patients } & \multicolumn{2}{|c|}{ Effect } & \multirow{2}{*}{ Quality } & \multirow{2}{*}{ Importance } \\
\hline \begin{tabular}{|} 
No of \\
studies
\end{tabular} & Design & $\begin{array}{c}\text { Risk of } \\
\text { bias }\end{array}$ & Inconsistency & Indirectness & Imprecision & $\begin{array}{c}\text { Other } \\
\text { considerations }\end{array}$ & \begin{tabular}{|c} 
Nonpenetrating \\
glaucoma \\
surgery plus \\
mitomycin C
\end{tabular} & $\begin{array}{c}\text { Trabeculectomy } \\
\text { plus mitomycin } \\
\text { C }\end{array}$ & $\begin{array}{c}\text { Relative } \\
(95 \% \\
\text { Cl) }\end{array}$ & Absolute & & \\
\hline \multicolumn{13}{|c|}{ PIO Reduction (better indicated by lower values) } \\
\hline 4 & $\begin{array}{l}\text { randomise } \\
\text { trials }\end{array}$ & $\begin{array}{l}\text { d no } \\
\text { serious } \\
\text { risk of } \\
\text { bias }\end{array}$ & $\begin{array}{l}\text { no serious } \\
\text { inconsistency }\end{array}$ & serious $^{1}$ & serious $^{2}$ & none & 120 & 120 & - & \begin{tabular}{|c|} 
WMD \\
2.36 \\
lower \\
$(10.55$ \\
lower to \\
5.84 \\
higher)
\end{tabular} & $\begin{array}{l}\text { Low } \\
\end{array}$ & CRITICAL \\
\hline \multicolumn{13}{|c|}{ Postoperative complications: shallow anterior chamber } \\
\hline 6 & $\begin{array}{l}\text { randomise } \\
\text { trials }\end{array}$ & d serious $^{3}$ & $\begin{array}{l}\text { no serious } \\
\text { inconsistency }\end{array}$ & \begin{tabular}{|l|} 
no serious \\
indirectness
\end{tabular} & serious ${ }^{2}$ & none & $\begin{array}{l}171 / 191 \\
(89.5 \%)\end{array}$ & $\begin{array}{l}61 / 190 \\
(32.1 \%)\end{array}$ & \begin{tabular}{|c|}
$\mathrm{RR}$ \\
0.31 \\
$(0.16$ \\
to 0.6$)$
\end{tabular} & \begin{tabular}{|c|}
222 \\
fewer \\
per \\
1.000 \\
(from \\
128 \\
fewer to \\
270
\end{tabular} & $\begin{array}{l}\square \square \square \\
\text { LOW }\end{array}$ & CRITICAL \\
\hline
\end{tabular}




\begin{tabular}{|c|c|c|c|c|c|c|c|c|c|c|c|c|}
\hline & & & & & & & & & & fewer) & & \\
\hline \multicolumn{13}{|c|}{ Postoperative complications: hypotony } \\
\hline 6 & $\begin{array}{l}\text { randomised } \\
\text { trials }\end{array}$ & serious ${ }^{3}$ & $\begin{array}{l}\text { no serious } \\
\text { inconsistency }\end{array}$ & $\begin{array}{l}\text { no serious } \\
\text { indirectness }\end{array}$ & serious $^{2}$ & none & $\begin{array}{l}19 / 191 \\
(9.9 \%)\end{array}$ & $\begin{array}{c}32 / 190 \\
(16.8 \%)\end{array}$ & $\begin{array}{c}\mathrm{RR} \\
0.47 \\
(0.16 \\
\text { to } \\
1.38)\end{array}$ & \begin{tabular}{|c|}
89 \\
fewer \\
per \\
1.000 \\
(from \\
141 \\
fewer to \\
64 \\
more)
\end{tabular} & \begin{tabular}{|l|} 
Lिएव \\
LOW
\end{tabular} & CRITICAL \\
\hline \multicolumn{13}{|c|}{ Postoperative complications: hyphema } \\
\hline 5 & $\begin{array}{l}\text { randomised } \\
\text { trials }\end{array}$ & serious ${ }^{3}$ & $\begin{array}{l}\text { no serious } \\
\text { inconsistency }\end{array}$ & $\mid \begin{array}{l}\text { no serious } \\
\text { indirectness }\end{array}$ & serious $^{2}$ & none & $\begin{array}{l}10 / 136 \\
(7.4 \%)\end{array}$ & $\begin{array}{c}20 / 134 \\
(14.9 \%)\end{array}$ & $\begin{array}{c}\mathrm{RR} \\
0.55 \\
(0.27 \\
\text { to } \\
1.11)\end{array}$ & \begin{tabular}{|c|}
67 \\
fewer \\
per \\
1.000 \\
(from \\
109 \\
fewer to \\
16 \\
more)
\end{tabular} & \begin{tabular}{|l|} 
पिएव \\
LOW
\end{tabular} & CRITICAL \\
\hline \multicolumn{13}{|c|}{ Postoperative complications: choroidal detachment } \\
\hline 3 & $\begin{array}{l}\text { randomised } \\
\text { trials }\end{array}$ & serious 3 & $\begin{array}{l}\text { no serious } \\
\text { inconsistency }\end{array}$ & \begin{tabular}{|l} 
no serious \\
indirectness
\end{tabular} & serious $^{2}$ & none & $\begin{array}{c}6 / 70 \\
(8.6 \%)\end{array}$ & $\begin{array}{c}11 / 69 \\
(15.9 \%)\end{array}$ & $\begin{array}{c}\mathrm{RR} \\
0.59 \\
(0.19 \\
\text { to } \\
1.82)\end{array}$ & \begin{tabular}{|c|}
65 \\
fewer \\
per \\
1.000 \\
(from \\
129 \\
fewer to
\end{tabular} & \begin{tabular}{|l|} 
पिएव \\
LOW
\end{tabular} & CRITICAL \\
\hline
\end{tabular}




\begin{tabular}{|l|l|l|l|l|l|l|l|c|c|}
\hline & & & & & & & & & \\
more $)$ & & \\
\hline
\end{tabular}

${ }^{1}$ IOP considered as a surrogate outcome of visual field loss in patient with open angle glaucoma. ${ }^{2}$ Small sample size, few events in the treatment and control groups.

3 The postoperative complications were calculated with both cohort and randomized clinical trial. 
4.4.2.2. Uso de antimetabolitos en la cirugía no penetrante

Question: Should Nonpenetrating Glaucoma Surgery plus Introperative Mitomycin C vs Nonpenetrating Glaucoma Surgery be used for Glaucoma?

Bibliography:

Cheng JW, Cai JP, Li Y, et al. Intraoperative Mitomycin C for Nonpenetrating Glaucoma Surgery. A sistematic review and Meta-analysis. J Glaucoma. 2011; 20(5): 322-6 C

Mielke V, Dawda K, Anand N. Deep sclerectomy and low dose mitomycin C: a randomised prospective trial in west Africa. Br J Ophthalmol. 2006; 90(3): 310-313.

Neudorfer M, Sadetzki S, Anisimova S, Geyer O. Nonpenetrating deep sclerectomy with the use of adjunctive mitomycin C. Ophthalmic Surg Lasers Imaging. 2004; 35(1): 6-12.

Kozobolis VP, Christodoulakis EV, Tzanakis N, Zacharopoulos I, Pallikaris IG. Primary deep sclerectomy versus primary deep sclerectomy with the use of mitomycin C in primary open-angle glaucoma. J Glaucoma. 2002; 11(4): 287-93.

\begin{tabular}{|c|c|c|c|c|c|c|c|c|c|c|c|c|}
\hline \multicolumn{7}{|c|}{ Quality assessment } & \multicolumn{2}{|c|}{ No of patients } & \multicolumn{2}{|c|}{ Effect } & \multirow[b]{2}{*}{ Quality } & \multirow[b]{2}{*}{ y Importance } \\
\hline $\begin{array}{c}\text { No of } \\
\text { studies }\end{array}$ & Design & $\begin{array}{c}\begin{array}{c}\text { Risk of } \\
\text { bias }\end{array} \\
\text { 年 }\end{array}$ & Inconsistency & Indirectness & Imprecision & $\begin{array}{c}\text { Other } \\
\text { considerations }\end{array}$ & $\begin{array}{c}\text { Nonpenetrating } \\
\text { Glaucoma } \\
\text { Surgery plus } \\
\text { Introperative } \\
\text { Mitomycin C }\end{array}$ & $\begin{array}{c}\text { Nonpenetrating } \\
\text { Glaucoma } \\
\text { Surgery }\end{array}$ & $\begin{array}{c}\text { Relative } \\
(95 \% \\
\mathrm{Cl})\end{array}$ & Absolute & & \\
\hline \multicolumn{13}{|c|}{ Percetage reduction in IOP (weighted mean difference): 12 months (better indicated by higher values) } \\
\hline 2 & $\begin{array}{l}\text { randomise } \\
\text { trials }\end{array}$ & serious & $\begin{array}{l}\text { no serious } \\
\text { inconsistency }\end{array}$ & serious $^{2}$ & serious $^{3}$ & none & 58 & 58 & - & \begin{tabular}{|c|}
7.75 \\
higher \\
$(2.66$ to \\
12.85 \\
higher $)^{4}$
\end{tabular} & \begin{tabular}{|l|l|} 
पि口प \\
VERY \\
LOW
\end{tabular} & CRITICAL \\
\hline
\end{tabular}




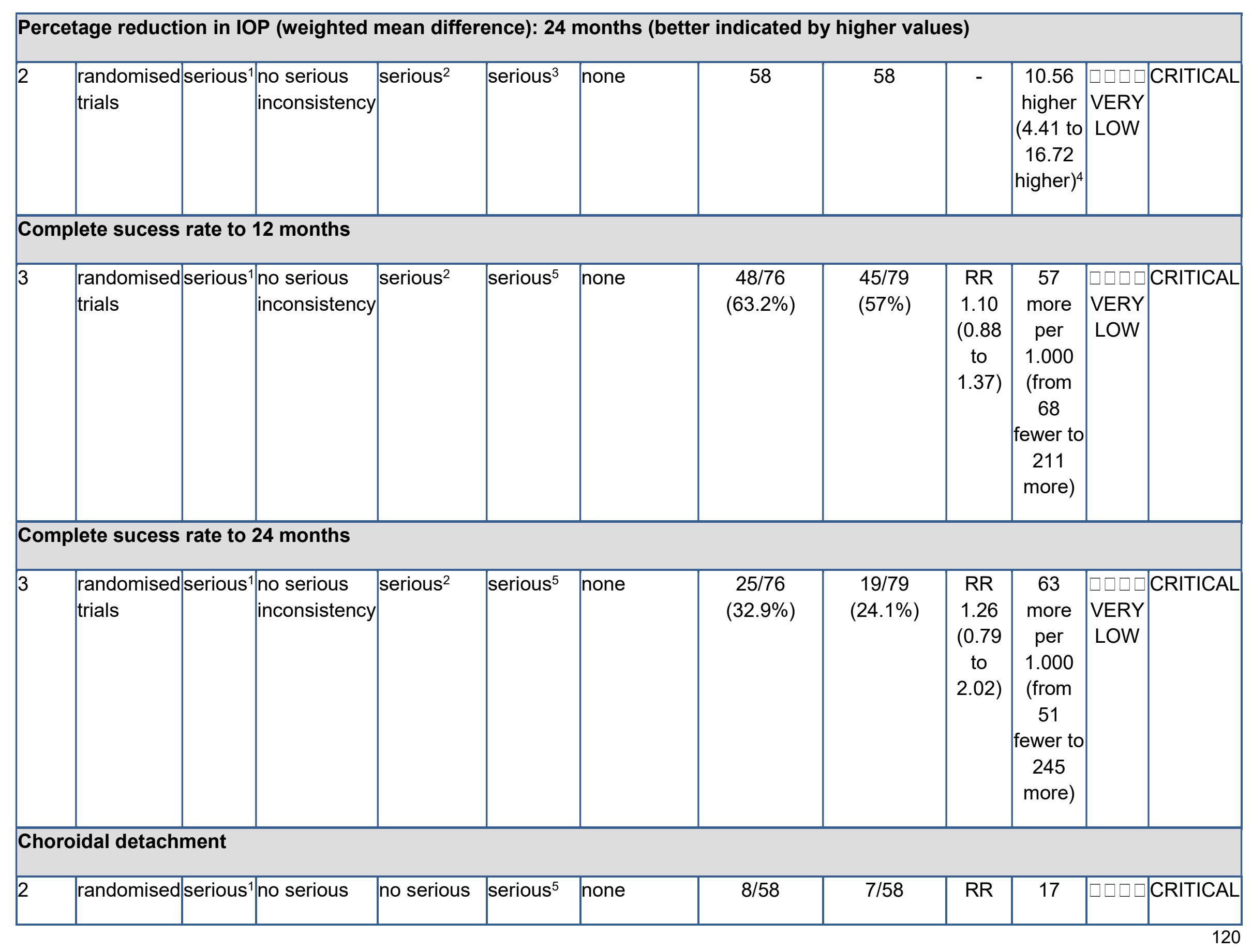




\begin{tabular}{|c|c|c|c|c|c|c|c|c|c|c|}
\hline & trials & inconsistency indirectness & & & $(13.8 \%)$ & $(12.1 \%)$ & $\begin{array}{c}1.14 \\
(0.45 \\
\text { to } \\
2.94)\end{array}$ & \begin{tabular}{|c|} 
more \\
per \\
1.000 \\
(from \\
66 \\
fewer to \\
234 \\
more)
\end{tabular} & LOW & \\
\hline \multicolumn{11}{|c|}{ Hyphema } \\
\hline 2 & $\left|\begin{array}{l}\text { randomised } \\
\text { trials }\end{array}\right| \begin{array}{l}\text { serious } \\
\text { tin } \\
\text { in }\end{array}$ & \begin{tabular}{|l|l|} 
no serious & no serious \\
inconsistency & indirectness
\end{tabular} & serious $^{5}$ & none & $\begin{array}{c}9 / 58 \\
(15.5 \%)\end{array}$ & $\begin{array}{c}6 / 58 \\
(10.3 \%)\end{array}$ & $\begin{array}{c}\mathrm{RR} \\
1.49 \\
(0.57 \\
\text { to } \\
3.93)\end{array}$ & \begin{tabular}{|c|}
51 \\
more \\
per \\
1.000 \\
(from \\
44 \\
fewer to \\
303 \\
more)
\end{tabular} & LOW & CRITICAL \\
\hline \multicolumn{11}{|c|}{ Leaks } \\
\hline 2 & \begin{tabular}{|l|l|l}
$\mid$ randomised & serious & \\
trials & & \\
in
\end{tabular} & \begin{tabular}{|l|l|} 
no serious & no serious \\
inconsistency & indirectness
\end{tabular} & serious $^{5,6}$ & none & $\begin{array}{c}3 / 63 \\
(4.8 \%)\end{array}$ & $\begin{array}{c}1 / 66 \\
(1.5 \%)\end{array}$ & $\begin{array}{c}\mathrm{RR} \\
2.44 \\
(0.37 \\
\text { to } \\
16.15)\end{array}$ & \begin{tabular}{|c|}
22 \\
more \\
per \\
1.000 \\
(from \\
10 \\
fewer to \\
230 \\
more)
\end{tabular} & LOW & CRITICAL \\
\hline
\end{tabular}




\begin{tabular}{|c|c|c|c|c|c|c|c|c|c|c|}
\hline 1 & \begin{tabular}{|l|l|} 
randomised serious \\
trials
\end{tabular} & $\begin{array}{l}\text { no serious } \\
\text { inconsistency }\end{array}$ & $\begin{array}{l}\text { no serious } \\
\text { indirectness }\end{array}$ & serious 5,6 none & $\begin{array}{c}2 / 13 \\
(15.4 \%)\end{array}$ & $\begin{array}{c}1 / 13 \\
(7.7 \%)\end{array}$ & \begin{tabular}{|c} 
RR 2 \\
$(0.21$ \\
to \\
$19.44)$
\end{tabular} & \begin{tabular}{|c}
77 \\
more \\
per \\
1.000 \\
(from \\
61 \\
fewer tc \\
1.000 \\
more)
\end{tabular} & $\begin{array}{ll}\text { LOप } \\
\text { LOW }\end{array}$ & CRITICAL \\
\hline
\end{tabular}

1 Allocation concealment and masking of outcome assessmente are not reported. 2 OAG progression as inferred from IOP.

${ }^{3}$ Total population size is less than 400 .

${ }^{4}$ Reduction in the IOP.

5 Total number of events is less than 300

${ }^{6}$ Wide confidence interval. 
4.4.2.3. Uso de implantes en la cirugía no penetrante

Question: Should NPGS with implant vs NPGS with no implant be used for open angle glaucoma? ${ }^{1}$

\section{Bibliography:}

Lake C, Dietlein TS, Jacobi PC, Konen W, Krieglstein GK. A prospective randomised trial of viscocanalostomy with and without implantation of a reticulated hyaluronic acid implant (SKGEL) in open angle glaucoma. Br J Ophthalmol. 2003 May; 87(5): 599-603.

Shaarawy T, Nguyen C, Schnyder C, Mermoud A. Comparative study between deep sclerectomy with and without collagen implant: long term follow up. Br J Ophthalmol. 2004 Jan; 88(1): 95-8. 3. In Shaarawy T, Mermoud A. Deep sclerectomy in one eye vs deep sclerectomy with collagen implant in the contralateral eye of the same patient: long-term follow-up. Eye (Lond). 2005 Mar; 19(3): 298-302.

\begin{tabular}{|c|c|c|c|c|c|c|c|c|c|c|c|c|}
\hline \multicolumn{7}{|c|}{ Quality assessment } & \multicolumn{2}{|c|}{ No of patients } & \multicolumn{2}{|c|}{ Effect } & \multirow{2}{*}{ Quality } & \multirow{2}{*}{ Importance } \\
\hline $\begin{array}{c}\text { No of } \\
\text { studies }\end{array}$ & Design & $\begin{array}{c}\text { Risk of } \\
\text { bias }\end{array}$ & Inconsistency & Indirectness & Imprecision & $\begin{array}{c}\text { Other } \\
\text { considerations }\end{array}$ & $\begin{array}{l}\text { NPGS } \\
\text { with } \\
\text { implant }\end{array}$ & $\begin{array}{c}\text { NPGS } \\
\text { with no } \\
\text { implant }\end{array}$ & $\begin{array}{l}\text { Relative } \\
(95 \% \text { Cl) }\end{array}$ & Absolute & & \\
\hline \multicolumn{13}{|c|}{ IOP (follow-up mean 12 months; better indicated by lower values) } \\
\hline 2 & $\begin{array}{l}\text { randomise } \\
\text { trials }\end{array}$ & serious $^{2}$ & serious $^{3}$ & serious $^{4}$ & serious $^{5}$ & none & 33 & 33 & - & \begin{tabular}{|c|} 
MD 2.27 \\
lower \\
$(7.66$ \\
lower to \\
3.12 \\
higher)
\end{tabular} & \begin{tabular}{|l|l|} 
पिएव \\
VERY \\
LOW
\end{tabular} & CRITICAL \\
\hline \multicolumn{13}{|c|}{ Percentage IOP reduction (follow-up mean $\mathbf{4 8}$ months; better indicated by lower values) } \\
\hline 2 & $\begin{array}{l}\text { randomise } \\
\text { trials }\end{array}$ & serious $^{2}$ & serious $^{3}$ & serious $^{6}$ & serious ${ }^{5}$ & none & 33 & 33 & - & $\begin{array}{c}\text { MD } 10.90 \\
\text { higher } \\
(18.64 \\
\text { lower to } \\
40.45\end{array}$ & 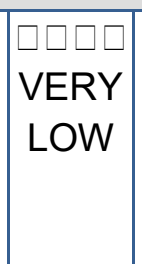 & CRITICAL \\
\hline
\end{tabular}




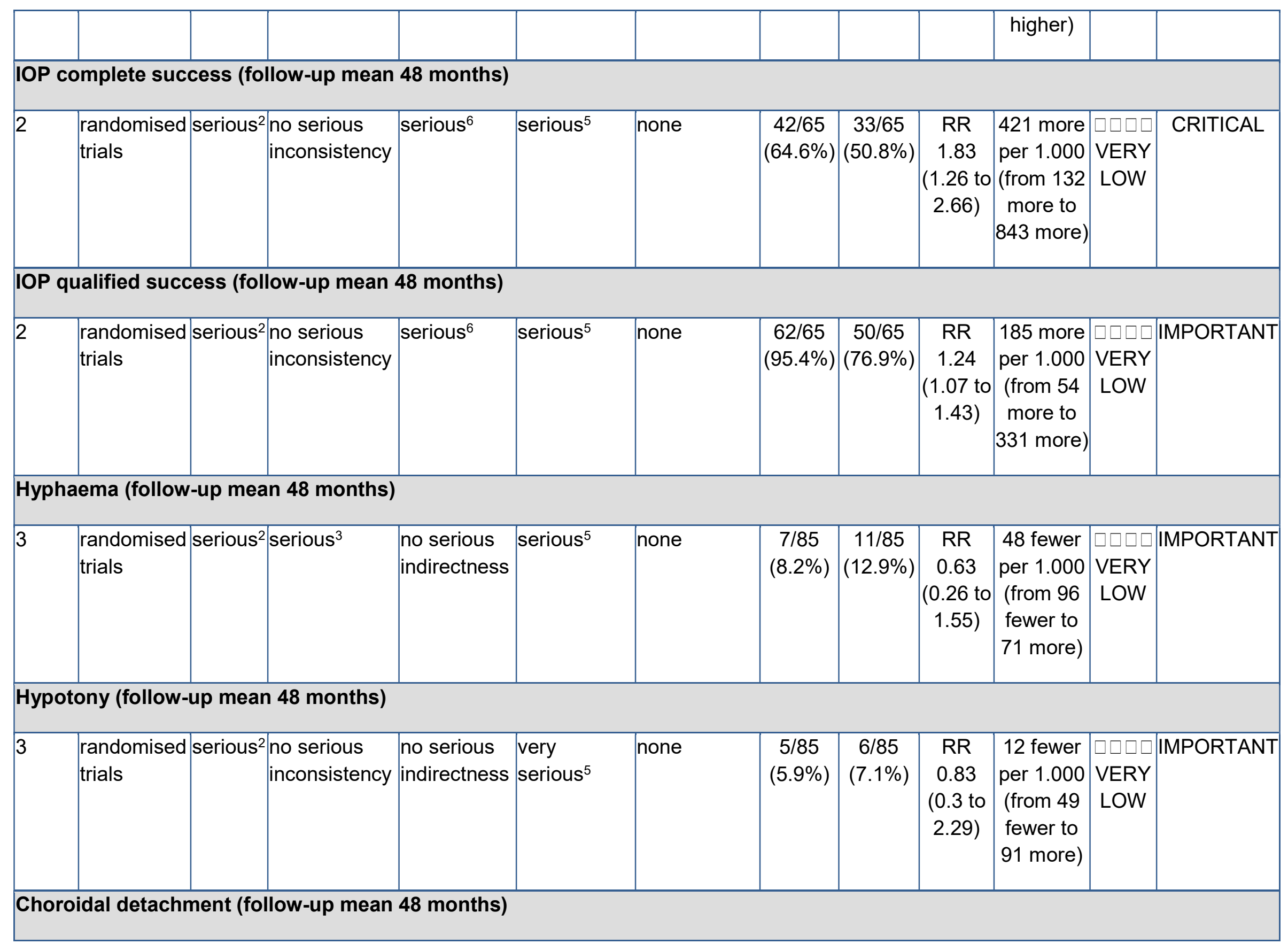




\begin{tabular}{|c|c|c|c|c|c|c|c|c|c|c|c|}
\hline 3 & \begin{tabular}{|l|l|}
$\begin{array}{l}\text { randomised } \\
\text { trials }\end{array}$ & \\
\end{tabular} & serious & \begin{tabular}{|l} 
no serious \\
indirectness
\end{tabular} & serious $^{5}$ & none & \begin{tabular}{|c|}
$7 / 85$ \\
$(8.2 \%)$
\end{tabular} & $\begin{array}{c}5 / 85 \\
(5.9 \%)\end{array}$ & \begin{tabular}{|c|} 
RR \\
1.33 \\
$(0.46$ to \\
$3.83)$
\end{tabular} & $\begin{array}{c}19 \text { more } \\
\text { per } 1.000 \\
\text { (from } 32 \\
\text { fewer to } \\
166 \text { more) }\end{array}$ & $\begin{array}{l}\square \square \square \\
\text { VERY } \\
\text { LOW }\end{array}$ & IMPORTANT \\
\hline \multicolumn{12}{|c|}{ Shadow anterior chamber (follow-up mean 48 months) } \\
\hline 3 & $\begin{array}{l}\text { randomised } \text { serious }^{2} \\
\text { trials }\end{array}$ & serious ${ }^{3}$ & \begin{tabular}{|l|} 
no serious \\
indirectness
\end{tabular} & serious $^{5}$ & none & \begin{tabular}{|c|}
$2 / 85$ \\
$(2.4 \%)$
\end{tabular} & $\begin{array}{c}3 / 85 \\
(3.5 \%)\end{array}$ & \begin{tabular}{|c|}
$\mathrm{RR}$ \\
0.67 \\
$(0.12$ to \\
$3.57)$
\end{tabular} & \begin{tabular}{|c|}
12 fewer \\
per 1.000 \\
(from 31 \\
fewer to \\
91 more)
\end{tabular} & $\begin{array}{l}\text { प्वप्र } \\
\text { VERY } \\
\text { LOW }\end{array}$ & IMPORTANT \\
\hline \multicolumn{12}{|c|}{ Cataract (follow-up mean $\mathbf{4 8}$ months) } \\
\hline 3 & $\begin{array}{l}\text { randomised } \\
\text { trials }\end{array}$ & serious ${ }^{3}$ & \begin{tabular}{|l} 
no serious \\
indirectness
\end{tabular} & $\begin{array}{l}\text { no serious } \\
\text { imprecision }\end{array}$ & none & \begin{tabular}{|c|}
$15 / 85$ \\
$(17.6 \%)$
\end{tabular} & $\begin{array}{c}14 / 85 \\
(16.5 \%)\end{array}$ & \begin{tabular}{|c|}
$\mathrm{RR}$ \\
1.05 \\
$(0.56$ to \\
$1.99)$
\end{tabular} & $\begin{array}{c}8 \text { more } \\
\text { per } 1.000 \\
\text { (from } 72 \\
\text { fewer to } \\
163 \text { more) }\end{array}$ & $\begin{array}{l}\text { पिएव } \\
\text { LOW }\end{array}$ & IMPORTANT \\
\hline \multicolumn{12}{|c|}{ Total complications (follow-up mean $\mathbf{4 8}$ months) } \\
\hline 3 & $\begin{array}{l}\text { randomised } \text { serious }^{2} \\
\text { trials }\end{array}$ & serious $^{3}$ & \begin{tabular}{|l} 
no serious \\
indirectness
\end{tabular} & serious $^{5}$ & none & \begin{tabular}{|c|}
$36 / 85$ \\
$(42.4 \%)$
\end{tabular} & $\begin{array}{c}39 / 85 \\
(45.9 \%)\end{array}$ & \begin{tabular}{|c|}
$R R$ \\
0.92 \\
$(0.66$ to \\
$1.29)$
\end{tabular} & $\begin{array}{c}37 \text { fewer } \\
\text { per } 1.000 \\
\text { (from } 156 \\
\text { fewer to } \\
133 \text { more) }\end{array}$ & $\begin{array}{l}\square \square \square \square \\
\text { VERY } \\
\text { LOW }\end{array}$ & CRITICAL \\
\hline
\end{tabular}

${ }^{1}$ NPGS Non penetrating glaucoma surgery

2 Intervention no blinded.

${ }^{3}$ High heterogeneity: high (= serious) or very high (= very serious).

${ }^{4}$ No explanation was provided. 
${ }^{5}$ Very small sample size, with low total number of events. Large confidence interval limits precision: includes appreciable benefit and appreciable harm of the intervention.

OAG progression as inferred from IOP

\section{MÉTODOS}

Como medida del efecto, para las variables dicotómicas se utilizó el cociente de riesgos (CR) y para las variables continuas se usó la diferencia de medias ponderadas (DMP), ambos con sus intervalos de confianza del 95\%. Como método estadístico se utilizaron, respectivamente, los métodos de Mantel-Haenszel y de la varianza inversa. Para el metanálisis se utilizó el modelo de efectos fijos, excepto cuando se encontró heterogeneidad significativa; en ese caso, se utilizó el modelo de efectos aleatorios.

\section{FOREST PLOTS}

\section{Media de la PIO a los 12 meses de seguimiento}

\begin{tabular}{|c|c|c|c|c|c|c|c|c|c|}
\hline \multirow[b]{2}{*}{ Study or Subgroup } & \multicolumn{3}{|c|}{ Experimental } & \multicolumn{3}{|c|}{ Control } & \multirow[b]{2}{*}{ Weight } & \multirow{2}{*}{$\begin{array}{l}\text { Mean Difference } \\
\mathrm{N} \text {, Random, } 95 \text { 品 } \mathrm{Cl}\end{array}$} & \multirow{2}{*}{$\begin{array}{l}\text { Mean Difference } \\
\mathrm{N}, \text { Random, } 95 \% \mathrm{Cl}\end{array}$} \\
\hline & Mean & SD & Total & Mean & SD 1 & Total & & & \\
\hline Luke 2003 & 17 & 3.1 & 20 & 16.5 & 5.8 & 20 & $49.6 \%$ & $0.50[-2.38,3.38]$ & 1 \\
\hline Shaarawy 2005 & 10.4 & 4 & 13 & 15.4 & 3 & 13 & $50.4 \%$ & $-5.00[-7.72,-2.28]$ & 민 \\
\hline Total $(95 \%$ Cl) & & & 33 & & & 33 & $100.0 \%$ & $-2.27[-7.66,3.12]$ & $\leftrightarrow$ \\
\hline $\begin{array}{l}\text { Heterogeneity: Tau² } \\
\text { Test for overall effect }\end{array}$ & $\begin{array}{l}13.08 ; C \\
Z=0.83\end{array}$ & & & & & & & & $\begin{array}{cccc}-100 & -50 & 1 & 1 \\
\text { Favours [control] } & \text { Favours [experimenta }\end{array}$ \\
\hline
\end{tabular}


Reducción porcentual de la PIO a partir de los valores basales

\begin{tabular}{|c|c|c|c|c|c|c|c|c|c|}
\hline \multirow[b]{2}{*}{ Study or Subgroup } & \multicolumn{3}{|c|}{ Experimental } & \multicolumn{3}{|c|}{ Control } & \multirow[b]{2}{*}{ Weight } & \multirow{2}{*}{$\begin{array}{l}\text { Mean Difference } \\
\mathrm{N}, \text { Random, } 95^{\circ} \mathrm{Cl} \\
\end{array}$} & \multirow{2}{*}{$\begin{array}{l}\text { Mean Difference } \\
\mathrm{N}, \text { Random, } 95 \% \text { Cl }\end{array}$} \\
\hline & Mean & SD & Total & Mean & SD & Total & & & \\
\hline Luke 2003 & 35.1 & 25.8 & 20 & 38.4 & 32.2 & 20 & $53.0 \%$ & $-3.30[-21.38,14.78]$ & \\
\hline Shaarawy 2005 & 60.5 & 28.5 & 13 & 33.6 & 31.6 & 13 & $47.0 \%$ & $26.90[3.77,50.03]$ & - \\
\hline Total (95\% Cl) & & & 33 & & & 33 & $100.0 \%$ & $10.90[-18.64,40.45]$ & \\
\hline $\begin{array}{l}\text { Heterogeneity: Taúz, } \\
\text { Test for overall effect }\end{array}$ & $\begin{array}{l}343.81 \\
Z=0.72\end{array}$ & & & & & & & & 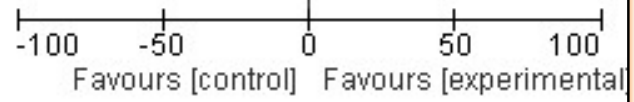 \\
\hline
\end{tabular}

\section{Tasa de éxito completo a los 48 meses}

\begin{tabular}{|c|c|c|c|c|c|c|c|c|}
\hline \multirow[b]{2}{*}{ Stucty or Subgroup } & \multicolumn{2}{|c|}{ Experimental } & \multicolumn{2}{|c|}{ Control } & \multirow[b]{2}{*}{ Weight } & \multirow{2}{*}{$\begin{array}{c}\text { Risk Ratio } \\
\text { M-H, Fixed, 95㭊 Cl }\end{array}$} & \multirow{2}{*}{\multicolumn{2}{|c|}{$\begin{array}{c}\text { Risk Ratio } \\
\text { M-H, Fixed, 95勄 Cl }\end{array}$}} \\
\hline & Events & Total & Events & Total & & & & \\
\hline Shaarawy 2004 & 33 & 52 & 18 & 52 & $78.3 \%$ & $1.83[1.20,2.81]$ & & \\
\hline Shaarawy 2005 & 9 & 13 & 5 & 13 & $21.7 \%$ & $1.80[0.83,3.92]$ & & \\
\hline Total $(95 \%$ Cl) & & 65 & & 65 & $100.0 \%$ & $1.83[1.26,2.66]$ & & 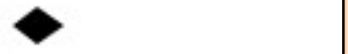 \\
\hline Total events & 42 & & 23 & & & & & \\
\hline $\begin{array}{l}\text { Heterogeneity: Chi } \\
\text { Test for overall effect }\end{array}$ & $\begin{array}{l}0.00, d f= \\
Z=3.15(\end{array}$ & $\begin{array}{l}(P=0 \\
=0.00\end{array}$ & $\begin{array}{l}\text { 97); } i^{2}=0 \\
\text { 2) }\end{array}$ & & & & $\begin{array}{cc}0.01 & 0.1 \\
& \text { Favours [control] }\end{array}$ & $\begin{array}{cc}10 & 100 \\
\text { Favours [experimental] }\end{array}$ \\
\hline
\end{tabular}

\section{Tasa de éxito calificado a los 48 meses}

\begin{tabular}{|c|c|c|c|c|c|c|c|c|}
\hline \multirow[b]{2}{*}{ Study or Subgroup } & \multicolumn{2}{|c|}{ Experimental } & \multicolumn{2}{|c|}{ Control } & \multirow[b]{2}{*}{ Weight } & \multirow{2}{*}{$\begin{array}{c}\text { Risk Ratio } \\
\text { M-H, Fixed, 95\% } \mathrm{Cl}\end{array}$} & \multirow{2}{*}{\multicolumn{2}{|c|}{$\begin{array}{c}\text { Risk Ratio } \\
\text { M-H, Fixed, } 95 \% \text { Cl }\end{array}$}} \\
\hline & Events & Total & Events & Total & & & & \\
\hline Shaarawy 2004 & 49 & 52 & 41 & 52 & $81.2 \%$ & $1.20[1.02,1.40]$ & & \\
\hline Shaarawy 2005 & 13 & 13 & 9 & 13 & $18.8 \%$ & $1.42[0.98,2.07]$ & & $=$ \\
\hline Total $(95 \%$ Cl) & & 65 & & 65 & $100.0 \%$ & $1.24[1.07,1.43]$ & & $\bullet$ \\
\hline \multirow{2}{*}{\multicolumn{7}{|c|}{$\begin{array}{l}\text { Total events } \\
\text { Heterogeneity: } C \mathrm{Ch}^{2}=0.72, \mathrm{df}=1(\mathrm{P}=0.40) ;\left.\right|^{2}=0 \%\end{array}$}} & & \\
\hline & & & & & & & $\begin{array}{l}0.01 \\
\quad \text { Favours [control] }\end{array}$ & $\begin{array}{cc}10 & 100 \\
\text { Favours [experimental] }\end{array}$ \\
\hline
\end{tabular}




\begin{tabular}{|c|c|c|c|c|c|c|c|c|}
\hline \multirow[b]{2}{*}{ Study or Subgroup } & \multicolumn{2}{|c|}{ Experimental } & \multicolumn{2}{|c|}{ Control } & \multirow[b]{2}{*}{ Weight } & \multirow{2}{*}{$\begin{array}{c}\text { Risk Ratio } \\
\text { M-H, Random, 95\% } \mathrm{Cl}\end{array}$} & \multirow{2}{*}{\multicolumn{2}{|c|}{$\begin{array}{c}\text { Risk Ratio } \\
\text { M-H, Random, 95\% } \mathrm{Cl}\end{array}$}} \\
\hline & Events & Total & Events & Total & & & & \\
\hline Luke 2003 & 2 & 20 & 3 & 20 & $28.8 \%$ & $0.67[0.12,3.57]$ & & \\
\hline Shaarawy 2004 & 4 & 52 & 8 & 52 & $62.8 \%$ & $0.50[0.16,1.56]$ & 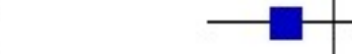 & - \\
\hline Shaarawy 2005 & 1 & 13 & 0 & 13 & $8.4 \%$ & $3.00[0.13,67.51]$ & & \\
\hline Total $\left(95^{\circ} \mathrm{Cl}\right)$ & & 85 & & 85 & $100.0^{0} \%$ & $0.63[0.26,1.55]$ & & \\
\hline Total events & 7 & & 11 & & & & & \\
\hline $\begin{array}{l}\text { Heterogeneity: Tau } \\
\text { Test for overall effect }\end{array}$ & $\begin{array}{l}0.00 ; \mathrm{Chi} \\
\mathrm{Z}=1.00\end{array}$ & $\begin{array}{l}=1.13, \\
=0.32\end{array}$ & & $=0.57$ & $; 1^{2}=0 \%$ & & $\begin{array}{l}0.010 .1 \\
\text { Favours [control] }\end{array}$ & $\begin{array}{|cc|}10 & 100 \\
\text { Favours [experimental }\end{array}$ \\
\hline
\end{tabular}

\section{Hipotonía}

\begin{tabular}{|c|c|c|c|c|c|c|c|c|c|}
\hline \multirow[b]{2}{*}{ Study or Subgroup } & \multicolumn{2}{|c|}{ Experimental } & \multicolumn{2}{|c|}{ Control } & \multirow[b]{2}{*}{ Weight } & $\begin{array}{c}\text { Risk Ratio } \\
\text { M-H. Random. 95听 CI }\end{array}$ & \multirow{2}{*}{\multicolumn{3}{|c|}{$\begin{array}{c}\text { Risk Ratio } \\
\text { M-H. Random. 95吹 Cl }\end{array}$}} \\
\hline & Events & Total & Events & Total & & M-H, Random, 95\% $\mathrm{Cl}$ & & & \\
\hline Luke 2003 & 5 & 20 & 6 & 20 & $100.0 \%$ & $0.83[0.30,2.29]$ & & 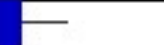 & \\
\hline Shaarawy 2004 & 0 & 52 & 0 & 52 & & Not estimable & & & \\
\hline Shaarawy 2005 & 0 & 13 & 0 & 13 & & Not estimable & & & \\
\hline Total (95吹 Cl) & & 85 & & 85 & $100.0 \%$ & $0.83[0.30,2.29]$ & & & \\
\hline Total events & 5 & & 6 & & & & & & \\
\hline $\begin{array}{l}\text { Heterogeneity: Not a } \\
\text { Test for overall effect }\end{array}$ & $\begin{array}{l}\text { plicable } \\
Z=0.35\end{array}$ & & & & & & $\begin{array}{cc}0.01 & 0.1 \\
\text { Favours [control }\end{array}$ & 1 Favours & 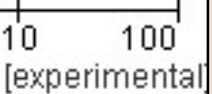 \\
\hline
\end{tabular}




\section{Desprendimiento coroideo}

\begin{tabular}{|c|c|c|c|c|c|c|c|}
\hline \multirow[b]{2}{*}{ Study or Subgroup } & \multicolumn{2}{|c|}{ Experimental } & \multicolumn{2}{|c|}{ Control } & \multirow[b]{2}{*}{ Weight } & \multirow{3}{*}{$\begin{array}{c}\text { Risk Ratio } \\
\text { M-H, Random, 95\% } \mathrm{Cl}\end{array}$} & \multirow{2}{*}{$\begin{array}{c}\text { Risk Ratio } \\
\text { M-H, Random, 95\% } \mathrm{Cl}\end{array}$} \\
\hline & Events & Total & Events & Total & & & \\
\hline Luke 2003 & 1 & 20 & 0 & 20 & $11.3 \%$ & & \\
\hline Shaarawy 2004 & 6 & 52 & 5 & 52 & $88.7 \%$ & $1.20[0.39,3.69]$ & \\
\hline Shaarawy 2005 & 0 & 13 & 0 & 13 & & Not estimable & \\
\hline Total $\left(95^{\circ}\right.$ 哗 & & 85 & & 85 & $100.0 \%$ & $1.33[0.46,3.83]$ & \\
\hline Total events & 7 & & 5 & & & & \\
\hline $\begin{array}{l}\text { Heterogeneity: Tau } \\
\text { Test for overall effect }\end{array}$ & $\begin{array}{l}0.00 ; \text { Chi }^{\prime} \\
Z=0.53(\end{array}$ & $\begin{array}{l}=0.29 \\
=0.60\end{array}$ & $d f=1(P$ & $=0.59)$ & $; I^{2}=0 \%$ & & 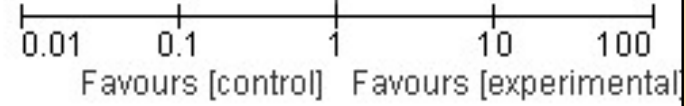 \\
\hline
\end{tabular}

\section{Cámara anterior superficial}

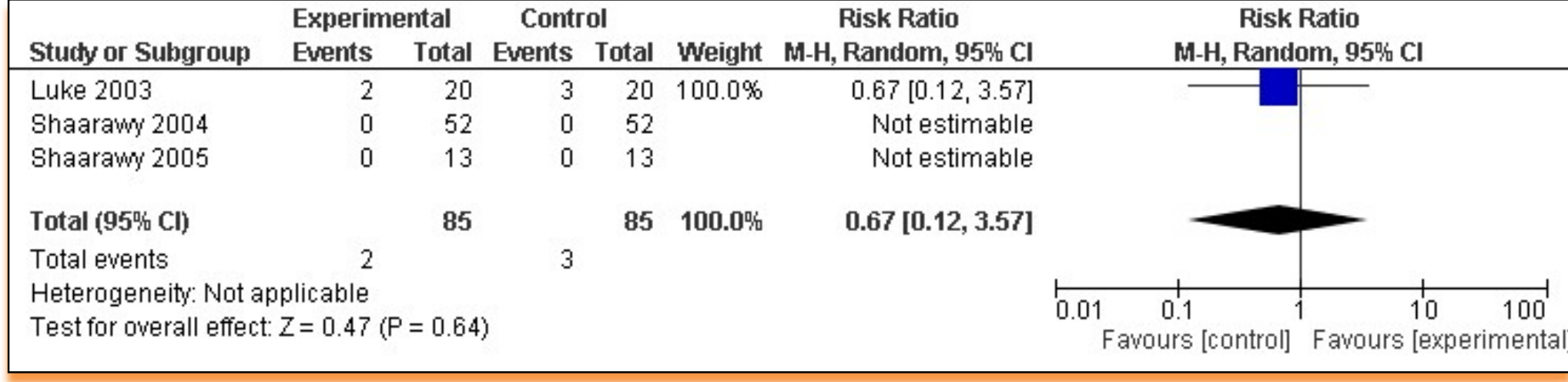


Catarata inducida por la cirugía o por progresión de la catarata preexistente

\begin{tabular}{|c|c|c|c|c|c|c|c|}
\hline \multirow[b]{2}{*}{ Study or Subgroup } & \multicolumn{2}{|c|}{ Experimental } & \multicolumn{2}{|c|}{ Control } & \multirow[b]{2}{*}{ Weight } & \multirow{2}{*}{$\begin{array}{c}\text { Risk Ratio } \\
\text { M-H, Random, 95\% Cl }\end{array}$} & \multirow{2}{*}{$\begin{array}{c}\text { Risk Ratio } \\
\text { M-H, Random, 95\% } \mathrm{Cl}\end{array}$} \\
\hline & Events & Total & Events & Total & & & \\
\hline Luke 2003 & 0 & 20 & 0 & 20 & & Not estimable & \\
\hline Shaarawy 2004 & 11 & 52 & 9 & 52 & $64.4 \%$ & $1.22[0.55,2.70]$ & \\
\hline Shaarawy 2005 & 4 & 13 & 5 & 13 & $35.6 \%$ & $0.80[0.28,2.32]$ & \\
\hline Total $(95 \%$ Cl) & & 85 & & 85 & $100.0 \%$ & $1.05[0.56,1.99]$ & \\
\hline Total events & 15 & & 14 & & & & \\
\hline $\begin{array}{l}\text { Heterogeneity: Tau }= \\
\text { Test for overall effect. }\end{array}$ & $\begin{array}{l}0.00 ; \text { Chi }^{\prime} \\
\mathrm{Z}=0.15\end{array}$ & $\begin{array}{l}=0.39, \\
=0.88\end{array}$ & $d f=1(P$ & $=0.53)$ & $; I^{2}=0 \%$ & & \begin{tabular}{|cc|cc|} 
& 1 & 1 & 1 \\
0.01 & 0.1 & 1 & 100 \\
& Favours [control] & Favours [experimentall
\end{tabular} \\
\hline
\end{tabular}

Total de complicaciones

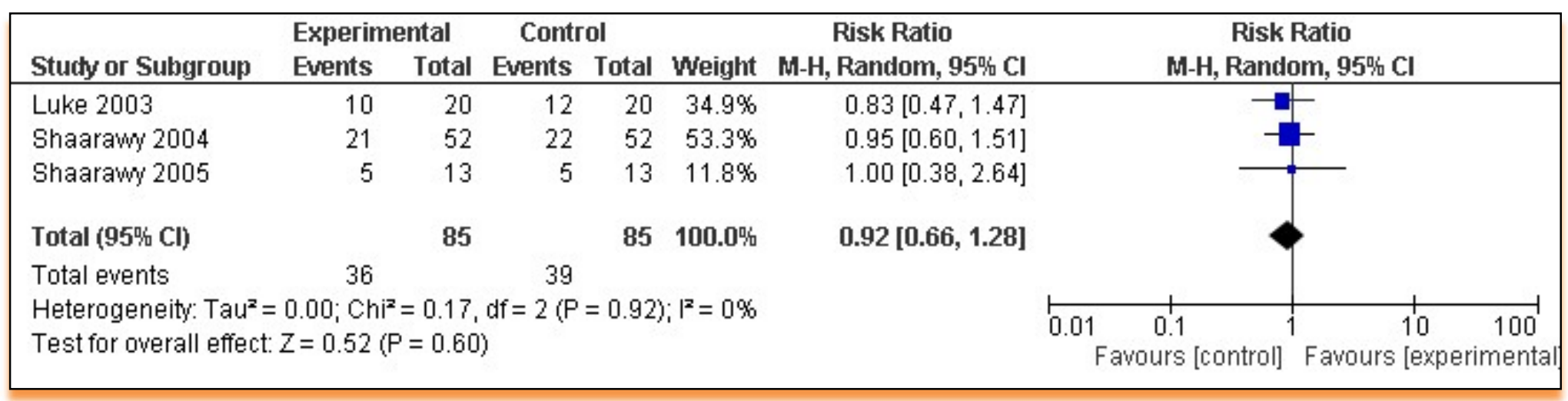


4.4.3. Uso de otras técnicas quirúrgicas/dispositivos en el tratamiento del glaucoma de ángulo abierto 4.4.3.1 Uso del implante Ex-PRESS en el tratamiento del glaucoma de ángulo abierto

Question: Should Ex-PRESS vs Trabeculectomy be used for Open-angle glaucoma?

Bibliography:

Wang W, Zhou M, Huang W, Zhang X. Ex-PRESS implantation versus trabeculectomy in uncontrolled glaucoma: a meta-analysis. PLoS One. 2013 May 31; 8(5): e63591.

Jong L, Lafuma A, Aguadé AS, Berdeaux G. Five-year extension of a clinical trial comparing the Ex-PRESS glaucoma filtration device and trabeculectomy in primary open-angle glaucoma. Clin Ophthalmol. 2011; 5: 527-33.

\begin{tabular}{|c|c|c|c|c|c|c|c|c|c|c|c|c|}
\hline \multicolumn{7}{|c|}{ Quality assessment } & \multicolumn{2}{|c|}{ No of patients } & \multicolumn{2}{|r|}{ Effect } & \multirow{2}{*}{ Quality } & \multirow{2}{*}{ Importance } \\
\hline \begin{tabular}{|c|}
$\begin{array}{c}\text { No of } \\
\text { studies }\end{array}$ \\
\end{tabular} & Design & Risk of bias & Inconsistency & Indirectness & Imprecision & $\begin{array}{c}\text { Other } \\
\text { considerations }\end{array}$ & EX-PRESST & rrabeculectomy & \begin{tabular}{|l|l|} 
Relative \\
$(95 \% \mathrm{Cl})$
\end{tabular} & Absolute & & \\
\hline \multicolumn{13}{|c|}{$\begin{array}{l}\text { Complete success rate (Wang 2013) (follow-up median } 5 \text { years; assessed with: target end-point IOP without antiglaucoma } \\
\text { medication) }\end{array}$} \\
\hline $5^{1}$ & $\begin{array}{l}\text { observational } \\
\text { studies }\end{array}$ & serious $^{1}$ & $\begin{array}{l}\text { no serious } \\
\text { inconsistency }{ }^{2}\end{array}$ & serious $^{3}$ & serious ${ }^{4}$ & none & \begin{tabular}{|l|}
$\mid 163 / 236$ \\
$(69.1 \%)$
\end{tabular} & $\begin{array}{l}176 / 258 \\
(68.2 \%)\end{array}$ & \begin{tabular}{|c|} 
OR \\
0.93 \\
$(0.39$ to \\
$2.23)$ \\
\end{tabular} & $\begin{array}{c}16 \text { fewer } \\
\text { per } 1.000 \\
\text { (from } 227 \\
\text { fewer to } \\
145 \text { more) }\end{array}$ & \begin{tabular}{|l|} 
पि口प \\
VERY \\
LOW
\end{tabular} & CRITICAL \\
\hline \multicolumn{13}{|c|}{$\begin{array}{l}\text { Complete success rate (de Jong 2011) (follow-up mean } 1 \text { years; assessed with: target end-point IOP without antiglaucoma } \\
\text { medication (final IOP < or }=18 \mathrm{~mm} \mathrm{Hg} \text { )) }\end{array}$} \\
\hline 1 & $\begin{array}{l}\text { randomised } \\
\text { trials }\end{array}$ & serious $^{5}$ & \begin{tabular}{|l} 
no serious \\
inconsistency
\end{tabular} & serious $^{3}$ & serious $^{4}$ & none & $\begin{array}{l}23 / 39 \\
(59 \%)\end{array}$ & $\begin{array}{c}18 / 39 \\
(46.2 \%)\end{array}$ & \begin{tabular}{|c|}
$\mathrm{RR}$ \\
1.68 \\
0.68 to
\end{tabular} & $\begin{array}{c}314 \text { more } \\
\text { per } 1.000 \\
\text { (from } 148 \\
\text { fewer to }\end{array}$ & \begin{tabular}{|l|l|} 
VERम \\
VERY \\
LOW
\end{tabular} & CRITICAL \\
\hline
\end{tabular}




\begin{tabular}{|c|c|c|c|c|c|c|c|c|c|c|c|c|}
\hline & & & & & & & & & 4.11) & $\begin{array}{l}1.000 \\
\text { more) }\end{array}$ & & \\
\hline \multicolumn{13}{|c|}{$\begin{array}{l}\text { Qualify success rate (Wang 2013) (follow-up median } 5 \text { years; assessed with: target end-point IOP with orwithout antiglaucoma } \\
\text { medication) }\end{array}$} \\
\hline $6^{1}$ & $\begin{array}{l}\text { observational } \\
\text { studies }\end{array}$ & serious $^{1}$ & serious $^{2}$ & serious $^{3}$ & serious $^{4}$ & none & $\begin{array}{l}181 / 216 \\
(83.8 \%)\end{array}$ & $\begin{array}{l}212 / 259 \\
(81.9 \%)\end{array}$ & \begin{tabular}{|c|} 
OR \\
1.00 \\
$(0.39$ to \\
$2.56)$
\end{tabular} & \begin{tabular}{|c|}
0 fewer \\
per 1.000 \\
(from 181 \\
fewer to \\
102 more)
\end{tabular} & \begin{tabular}{|l|} 
QRिए \\
VERY \\
LOW
\end{tabular} & CRITICAL \\
\hline \multicolumn{13}{|c|}{$\begin{array}{l}\text { Qualify success rate (de Jong 2011) (follow-up mean } 1 \text { years; assessed with: target end-point IOP with or without antiglaucoma } \\
\text { medication (final IOP < or }=18 \mathrm{~mm} \mathrm{Hg} \text { ) }\end{array}$} \\
\hline 1 & $\begin{array}{l}\text { randomised } \\
\text { trials }\end{array}$ & serious $^{5}$ & \begin{tabular}{|l|l} 
no serious \\
inconsistency
\end{tabular} & serious $^{3}$ & serious $^{4}$ & none & $\left|\begin{array}{c}38 / 39 \\
(97.4 \%)\end{array}\right|$ & $\begin{array}{c}39 / 39 \\
(100 \%)\end{array}$ & \begin{tabular}{|c|}
$\mathrm{RR}$ \\
0.33 \\
$(0.01$ to \\
$8.22)$
\end{tabular} & \begin{tabular}{|c|}
670 fewer \\
per 1.000 \\
(from 990 \\
fewer to \\
1.000 \\
more)
\end{tabular} & \begin{tabular}{|l|} 
GQपि \\
VERY \\
LOW
\end{tabular} & CRITICAL \\
\hline \multicolumn{13}{|c|}{ Adverse event: hypotony (follow-up mean 9-65.6 months) } \\
\hline 71 & $\begin{array}{l}\text { observational } \\
\text { studies }\end{array}$ & serious $^{1}$ & serious $^{2}$ & \begin{tabular}{|l} 
no serious \\
indirectness
\end{tabular} & serious $^{4}$ & none & \begin{tabular}{|c|}
$26 / 246$ \\
$(10.6 \%)$
\end{tabular} & $\begin{array}{c}74 / 289 \\
(25.6 \%)\end{array}$ & $\mid \begin{array}{c}\text { OR } \\
0.29 \\
(0.13 \text { to } \\
0.65)\end{array}$ & $\begin{array}{c}165 \text { fewer } \\
\text { per } 1.000 \\
\text { (from } 73 \\
\text { fewer to } \\
213 \text { fewer) }\end{array}$ & \begin{tabular}{|l|}
$\square \square \square \square$ \\
VERY \\
LOW
\end{tabular} & CRITICAL \\
\hline
\end{tabular}




\begin{tabular}{|c|c|c|c|c|c|c|c|c|c|c|c|c|}
\hline \multicolumn{13}{|c|}{ Adverse event: choroidal effusion (follow-up mean 9-65.6 months) } \\
\hline $6^{1}$ & $\begin{array}{l}\text { observational } \\
\text { studies }\end{array}$ & \begin{tabular}{|l} 
no \\
serious \\
risk of \\
bias $^{1}$
\end{tabular} & serious $^{2}$ & \begin{tabular}{|l|} 
no serious \\
indirectness
\end{tabular} & serious $^{4}$ & none & \begin{tabular}{|l|}
$24 / 231$ \\
$(10.4 \%)$
\end{tabular} & $\begin{array}{c}46 / 274 \\
(16.8 \%)\end{array}$ & \begin{tabular}{|c|}
$R R$ \\
0.65 \\
$(0.24$ to \\
$1.8)$
\end{tabular} & \begin{tabular}{|c|}
59 fewer \\
per 1.000 \\
(from 128 \\
fewer to \\
134 more)
\end{tabular} & \begin{tabular}{|l|l|} 
पिएव \\
VERY \\
LOW
\end{tabular} & CRITICAL \\
\hline \multicolumn{13}{|c|}{ Adverse event: flat anterior chamber (follow-up mean 9-65.6 months) } \\
\hline 5 & $\begin{array}{l}\text { observational } \\
\text { studies }\end{array}$ & \begin{tabular}{|l} 
no \\
serious \\
risk of \\
bias $^{1}$
\end{tabular} & serious $^{2}$ & \begin{tabular}{|l|} 
no serious \\
indirectness
\end{tabular} & serious $^{4}$ & none & \begin{tabular}{c|}
$9 / 190$ \\
$(4.7 \%)$
\end{tabular} & $\begin{array}{l}8 / 192 \\
(4.2 \%)\end{array}$ & \begin{tabular}{|c|} 
RR \\
1.06 \\
$(0.36$ to \\
$3.07)$
\end{tabular} & \begin{tabular}{|c|}
2 more \\
per 1.000 \\
(from 27 \\
fewer to \\
86 more)
\end{tabular} & \begin{tabular}{|l|l|} 
पिएव \\
VERY \\
LOW
\end{tabular} & CRITICAL \\
\hline \multicolumn{13}{|c|}{ Adverse event: hyphema (follow-up mean 9-65.6 months) } \\
\hline 7 & $\begin{array}{l}\text { observational } \\
\text { studies }\end{array}$ & serious $^{1}$ & serious $^{2}$ & \begin{tabular}{|l|} 
no serious \\
indirectness
\end{tabular} & serious $^{4}$ & none & \begin{tabular}{c|}
$4 / 249$ \\
$(1.6 \%)$
\end{tabular} & $\begin{array}{l}20 / 271 \\
(7.4 \%)\end{array}$ & \begin{tabular}{|c|}
$\mathrm{RR}$ \\
0.36 \\
$(0.13$ to \\
$0.97)$
\end{tabular} & \begin{tabular}{|c|}
47 fewer \\
per 1.000 \\
(from 2 \\
fewer to \\
64 fewer)
\end{tabular} & 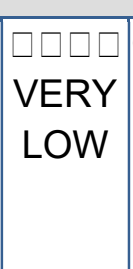 & CRITICAL \\
\hline \multicolumn{13}{|c|}{ Adverse event: bleb leak (follow-up mean 9-65.6 months) } \\
\hline 1 & $\begin{array}{l}\text { observational } \\
\text { studies }\end{array}$ & serious $^{1}$ & serious $^{2}$ & \begin{tabular}{|l|} 
no serious \\
indirectness
\end{tabular} & serious $^{4}$ & none & \begin{tabular}{|l|}
$38 / 226$ \\
$(16.8 \%)$
\end{tabular} & $\begin{array}{c}34 / 249 \\
(13.7 \%)\end{array}$ & \begin{tabular}{|c|}
$\mathrm{RR}$ \\
1.41 \\
$(0.84$ to \\
$2.39)$
\end{tabular} & \begin{tabular}{|c|}
56 more \\
per 1.000 \\
(from 22 \\
fewer to \\
190 more)
\end{tabular} & \begin{tabular}{|l|} 
पिएव \\
VERY \\
LOW
\end{tabular} & CRITICAL \\
\hline \multicolumn{13}{|c|}{ Adverse event: endophthalmitis (follow-up mean 9-65.6 months) } \\
\hline 1 & observational & serious $^{1}$ & serious $^{2}$ & no serious & serious $^{4}$ & none & $1 / 60$ & $1 / 61$ & $\begin{array}{l}R R \\
1.04\end{array}$ & \begin{tabular}{|c|}
1 more \\
per 1.000
\end{tabular} & \begin{tabular}{|l|} 
पि口 \\
VERY
\end{tabular} & CRITICAL \\
\hline
\end{tabular}




\begin{tabular}{|c|c|c|c|c|c|c|}
\hline studies & indirectness & $(1.7 \%)$ & $(1.6 \%)$ & \begin{tabular}{|l|} 
(0.1 to \\
$10.49)$
\end{tabular} & $\begin{array}{c}\text { (from } 15 \\
\text { fewer to } \\
156 \text { more) }\end{array}$ & LOW \\
\hline
\end{tabular}

Observational studies and/or RCTstudies.

High heterogeneity. Different studies design.

IOP considered as a surrogate outcome of visual field loss in patient with open angle glaucoma

Wide $95 \%$ interval confidence and/or includes potential benefit or, potential harm of the intervention

5 Open study: the physician knew which treatment was given when measuring intraocular pressure. The study was limited to one center and surgeon limiting the external validity. Patients were operated on one eye only, which introduces some interactions between nonoperated eye and operated eye. The sample size was fixed ad hoc.

4.4.3.2. Uso de dispositivos de drenaje (valvulados o no) en el tratamiento del glaucoma de ángulo Question: Should trabeculectomy vs Ahmed implant be used for open angle glaucoma?

Bibliography:

Minckler DS, Vedula SS, Li TJ, et al. Aqueous shunts for glaucoma. Cochrane Database Syst Rev 2006; (2): CD004918.

Minckler DS, Francis BA, Hodapp EA, Jampel HD, Lin SC, Samples JR, Smith SD, Singh K. Aqueous Shunts in Glaucoma. A Report by the AmericanAcademy of Ophthalmology. Ophthalmology 2008; 115: 1089-98. 


\begin{tabular}{|c|c|c|c|c|c|c|c|c|c|c|c|c|}
\hline \multicolumn{7}{|c|}{ Quality assessment } & \multicolumn{2}{|c|}{ No of patients } & \multicolumn{2}{|r|}{ Effect } & \multirow{2}{*}{ Quality } & \multirow{2}{*}{ Importance } \\
\hline $\begin{array}{c}\text { No of } \\
\text { studies }\end{array}$ & Design & $\begin{array}{l}\text { Risk of } \\
\text { bias }\end{array}$ & Inconsistency & Indirectness & s Imprecision & $\begin{array}{c}\text { Other } \\
\text { considerations }\end{array}$ & Trabeculectomy & Ahmed & $\begin{array}{c}\text { Relative } \\
(95 \% \\
\mathrm{Cl})\end{array}$ & Absolute & & \\
\hline \multicolumn{13}{|c|}{ Mean intraocular pressure at 11 to 13 months follow up (better indicated by lower values) } \\
\hline 2 & $\begin{array}{l}\text { randomised } \\
\text { trials }\end{array}$ & serious $^{1}$ & serious $^{2}$ & serious $^{3}$ & serious ${ }^{4}$ & none & 103 & 92 & - & $\begin{array}{c}\text { MD } 3.81 \\
\text { lower }(5.69 \\
\text { to } 1.94 \\
\text { lower) }\end{array}$ & \begin{tabular}{|l|l|} 
पि口प \\
VERY \\
LOW
\end{tabular} & CRITICAL \\
\hline
\end{tabular}

1 Did not specify methods of allocation concealment and blinding.

${ }^{2}$ I-squared value of $53 \%$ indicates statistical heterogeneity.

${ }^{3}$ OAG progression as inferred from IOP.

${ }^{4}$ Small sample.

${ }^{5}$ Measured by $\mathrm{mm} \mathrm{Hg}$

Question: Should Baerveldt glaucoma implant vs trabeculectomy be used for open angle glaucoma?

Bibliography:

Gedde SJ, Schiffman JC, Feuer WJ, Herndon LW, Brandt JD, Budenz DL. Treatment Outcomes in the Tube Versus Trabeculectomy (TVT) Study After Five Years of Follow-up. Am J Ophthalmol 2012; 153: 789-803.

Gedde SJ, Herndon LW, Brandt JD, Budenz DL, Feuer WJ, Schiffman JC. Postoperative Complications in the Tube Versus Trabeculectomy (TVT) Study During Five Years of Follow-up. Am J Ophthalmol 2012; 153(5): 804-14. 


\begin{tabular}{|c|c|c|c|c|c|c|c|c|c|c|c|c|}
\hline \multicolumn{7}{|c|}{ Quality assessment } & \multicolumn{2}{|c|}{ No of patients } & \multicolumn{2}{|c|}{ Effect } & \multirow{2}{*}{ Quality } & \multirow{2}{*}{ Importance } \\
\hline $\begin{array}{c}\text { No of } \\
\text { studies }\end{array}$ & Design & $\begin{array}{c}\text { Risk of } \\
\text { bias }\end{array}$ & Inconsistency & Indirectness & Imprecision & $\begin{array}{c}\text { Other } \\
\text { considerations }\end{array}$ & $\begin{array}{c}\text { Baerveldt } \\
\text { glaucoma } \\
\text { implant }\end{array}$ & Trabeculectomy & $\mid \begin{array}{l}\text { Relative } \\
(95 \% \mathrm{Cl})\end{array}$ & Absolute & & \\
\hline \multicolumn{13}{|c|}{ Intraocular pressure at 1 year follow up (better indicated by lower values) } \\
\hline 1 & $\begin{array}{l}\text { randomised } \\
\text { trials }\end{array}$ & serious $^{1}$ & $\begin{array}{l}\text { no serious } \\
\text { inconsistency }\end{array}$ & serious $^{2}$ & serious $^{3}$ & none & 0 & - & - & $\begin{array}{c}\text { not } \\
\text { pooled }\end{array}$ & \begin{tabular}{|l|} 
पिपा \\
VERY \\
LOW
\end{tabular} & CRITICAL \\
\hline \multicolumn{13}{|c|}{ Intraocular pressure at 5 year follow up (better indicated by lower values) } \\
\hline 1 & $\begin{array}{l}\text { randomised } \\
\text { trials }\end{array}$ & serious $^{1}$ & $\begin{array}{l}\text { no serious } \\
\text { inconsistency }\end{array}$ & serious $^{2}$ & serious $^{3}$ & none & 0 & - & - & $\begin{array}{c}\text { not } \\
\text { pooled }\end{array}$ & \begin{tabular}{|l|} 
VIDI \\
VERY \\
LOW
\end{tabular} & CRITICAL \\
\hline \multicolumn{13}{|c|}{ Treatment failure } \\
\hline 1 & $\begin{array}{l}\text { randomised } \\
\text { trials }\end{array}$ & serious $^{1}$ & $\begin{array}{l}\text { no serious } \\
\text { inconsistency }\end{array}$ & serious $^{2}$ & serious $^{4}$ & none & \begin{tabular}{|c|}
$24 / 73$ \\
$(32.9 \%)$
\end{tabular} & $\begin{array}{l}42 / 84 \\
(50 \%)\end{array}$ & \begin{tabular}{|c} 
RR \\
0.39 \\
$(0.28$ to \\
$0.56)$
\end{tabular} & \begin{tabular}{|c|}
305 \\
fewer per \\
1.000 \\
(from 220 \\
fewer to \\
360 \\
fewer)
\end{tabular} & \begin{tabular}{|l|} 
VER \\
VERY \\
LOW
\end{tabular} & CRITICAL \\
\hline \multicolumn{13}{|c|}{ Complete success } \\
\hline 1 & \begin{tabular}{|l} 
randomised \\
trials
\end{tabular} & serious $^{1}$ & \begin{tabular}{|l|} 
no serious \\
inconsistency
\end{tabular} & serious ${ }^{2}$ & serious $^{4}$ & none & \begin{tabular}{|c|}
$18 / 73$ \\
$(24.7 \%)$
\end{tabular} & $\begin{array}{c}24 / 84 \\
(28.6 \%)\end{array}$ & \begin{tabular}{|c} 
RR \\
0.86 \\
$(0.51$ to \\
$1.46)$
\end{tabular} & \begin{tabular}{|c|}
40 fewer \\
per 1.000 \\
from 140 \\
fewer to
\end{tabular} & \begin{tabular}{|l|} 
पिप्र \\
VERY \\
LOW
\end{tabular} & CRITICAL \\
\hline
\end{tabular}




\begin{tabular}{|c|c|c|c|c|c|c|c|c|c|c|c|c|}
\hline & & & & & & & & & & $\begin{array}{c}131 \\
\text { more) }\end{array}$ & & \\
\hline \multicolumn{13}{|c|}{ Qualified success } \\
\hline 1 & $\begin{array}{l}\text { randomised } \\
\text { trials }\end{array}$ & serious $^{1}$ & $\begin{array}{l}\text { no serious } \\
\text { inconsistency }\end{array}$ & serious $^{2}$ & serious $^{4}$ & none & \begin{tabular}{|c|}
$31 / 73$ \\
$(42.5 \%)$
\end{tabular} & $\begin{array}{c}18 / 84 \\
(21.4 \%)\end{array}$ & \begin{tabular}{|c}
$\mathrm{RR}$ \\
1.98 \\
$(1.22$ to \\
$3.23)$
\end{tabular} & \begin{tabular}{|c|}
210 more \\
per 1.000 \\
(from 47 \\
more to \\
478 \\
more)
\end{tabular} & \begin{tabular}{|l|} 
पि口प \\
VERY \\
LOW
\end{tabular} & CRITICAL \\
\hline \multicolumn{13}{|c|}{ Total number of patients early postoperative complications } \\
\hline 1 & $\begin{array}{l}\text { randomised } \\
\text { trials }\end{array}$ & serious $^{1}$ & $\begin{array}{l}1 \text { no serious } \\
\text { inconsistency }\end{array}$ & $\begin{array}{l}\text { no serious } \\
\text { indirectness }\end{array}$ & serious $^{4}$ & none & \begin{tabular}{|c|}
$22 / 107$ \\
$(20.6 \%)$
\end{tabular} & $\begin{array}{l}39 / 105 \\
(37.1 \%)\end{array}$ & \begin{tabular}{|c} 
RR \\
0.55 \\
$(0.35$ to \\
$0.87)$
\end{tabular} & \begin{tabular}{|c}
167 \\
fewer per \\
1.000 \\
(from 48 \\
fewer to \\
241 \\
fewer)
\end{tabular} & \begin{tabular}{|l|} 
पिएव \\
LOW
\end{tabular} & CRITICAL \\
\hline \multicolumn{13}{|c|}{ Chroidal effusion } \\
\hline 1 & $\begin{array}{l}\text { randomised } \\
\text { trials }\end{array}$ & serious $^{1}$ & $\begin{array}{l}1 \text { no serious } \\
\text { inconsistency }\end{array}$ & $\begin{array}{l}\text { no serious } \\
\text { indirectness }\end{array}$ & serious $^{4}$ & none & $\begin{array}{l}15 / 107 \\
(14 \%)\end{array}$ & $\begin{array}{c}14 / 105 \\
(13.3 \%)\end{array}$ & \begin{tabular}{|c|} 
RR \\
1.05 \\
$(0.53$ to \\
$2.07)$
\end{tabular} & \begin{tabular}{|c|}
7 more \\
per 1.000 \\
(from 63 \\
fewer to \\
143 \\
more)
\end{tabular} & \begin{tabular}{|l|} 
Lिएव \\
LOW
\end{tabular} & CRITICAL \\
\hline \multicolumn{13}{|c|}{ Shallow or flat anterior chamber } \\
\hline 1 & \begin{tabular}{|l} 
randomised \\
trials
\end{tabular} & serious $^{1}$ & $\begin{array}{l}1 \text { no serious } \\
\text { inconsistency }\end{array}$ & $\begin{array}{l}\text { no serious } \\
\text { indirectness }\end{array}$ & serious ${ }^{4}$ & none & \begin{tabular}{|c|}
$11 / 107$ \\
$(10.3 \%)$
\end{tabular} & $\begin{array}{l}10 / 105 \\
(9.5 \%)\end{array}$ & \begin{tabular}{|c|}
$R R$ \\
1.08 \\
$(0.48$ to
\end{tabular} & \begin{tabular}{|c|}
8 more \\
per 1.000 \\
(from 50
\end{tabular} & \begin{tabular}{|l|} 
LOप \\
LOW
\end{tabular} & CRITICAL \\
\hline
\end{tabular}




\begin{tabular}{|c|c|c|c|c|c|c|c|c|c|c|c|c|}
\hline & & & & & & & & & 2.43) & $\begin{array}{c}\text { fewer to } \\
136 \\
\text { more) }\end{array}$ & & \\
\hline \multicolumn{13}{|c|}{ Wound leak } \\
\hline 1 & \begin{tabular}{|l|} 
randomised \\
trials
\end{tabular} & serious 1 & \begin{tabular}{|l|} 
no serious \\
inconsistency
\end{tabular} & \begin{tabular}{|l|} 
no serious \\
indirectness
\end{tabular} & serious $^{4}$ & none & \begin{tabular}{|c|}
$1 / 107$ \\
$(0.93 \%)$
\end{tabular} & $\begin{array}{c}12 / 105 \\
(11.4 \%)\end{array}$ & \begin{tabular}{|c}
$\mathrm{RR}$ \\
0.08 \\
$(0.01$ to \\
$0.62)$
\end{tabular} & $\begin{array}{c}105 \\
\text { fewer per } \\
1.000 \\
\text { (from } 43 \\
\text { fewer to } \\
113 \\
\text { fewer) }\end{array}$ & LOW & CRITICAL \\
\hline \multicolumn{13}{|c|}{ Hyphema } \\
\hline 1 & \begin{tabular}{|l|} 
randomised \\
trials
\end{tabular} & serious 1 & \begin{tabular}{|l|} 
no serious \\
inconsistency
\end{tabular} & $\begin{array}{l}\text { no serious } \\
\text { indirectness }\end{array}$ & serious $^{4}$ & none & $\begin{array}{l}2 / 107 \\
(1.9 \%)\end{array}$ & $\begin{array}{l}8 / 105 \\
(7.6 \%)\end{array}$ & \begin{tabular}{|c}
$\mathrm{RR}$ \\
0.25 \\
$(0.05$ to \\
$1.13)$
\end{tabular} & \begin{tabular}{|l|}
57 fewer \\
per 1.000 \\
(from 72 \\
fewer to \\
10 more)
\end{tabular} & LOW & CRITICAL \\
\hline \multicolumn{13}{|c|}{ Total number of patients late postoperative complications } \\
\hline 1 & \begin{tabular}{|l|} 
randomised \\
trials
\end{tabular} & serious 1 & $\begin{array}{l}\text { no serious } \\
\text { inconsistency }\end{array}$ & $\begin{array}{l}\text { no serious } \\
\text { indirectness }\end{array}$ & serious $^{4}$ & none & \begin{tabular}{|c|}
$36 / 107$ \\
$(33.6 \%)$
\end{tabular} & $\begin{array}{c}38 / 105 \\
(36.2 \%)\end{array}$ & \begin{tabular}{|c|}
$R R$ \\
0.93 \\
$(0.64$ to \\
$1.34)$
\end{tabular} & \begin{tabular}{|c|}
25 fewer \\
per 1.000 \\
(from 130 \\
fewer to \\
123 \\
more)
\end{tabular} & $\begin{array}{l}\text { LOप } \\
\text { LOW }\end{array}$ & CRITICAL \\
\hline \multicolumn{13}{|c|}{ Persistent corneal edema } \\
\hline 1 & \begin{tabular}{|l|} 
randomised \\
trials
\end{tabular} & serious 1 & \begin{tabular}{|l|} 
no serious \\
inconsistency
\end{tabular} & $\mid \begin{array}{l}\text { no serious } \\
\text { indirectness }\end{array}$ & serious ${ }^{4}$ & none & \begin{tabular}{|c|}
$17 / 107$ \\
$(15.9 \%)$
\end{tabular} & $\begin{array}{l}9 / 105 \\
(8.6 \%)\end{array}$ & \begin{tabular}{|c|} 
RR \\
1.85 \\
$(0.87$ to
\end{tabular} & \begin{tabular}{|c|}
73 more \\
per 1.000 \\
(from 11
\end{tabular} & \begin{tabular}{|l|} 
L००ण \\
LOW
\end{tabular} & CRITICAL \\
\hline
\end{tabular}




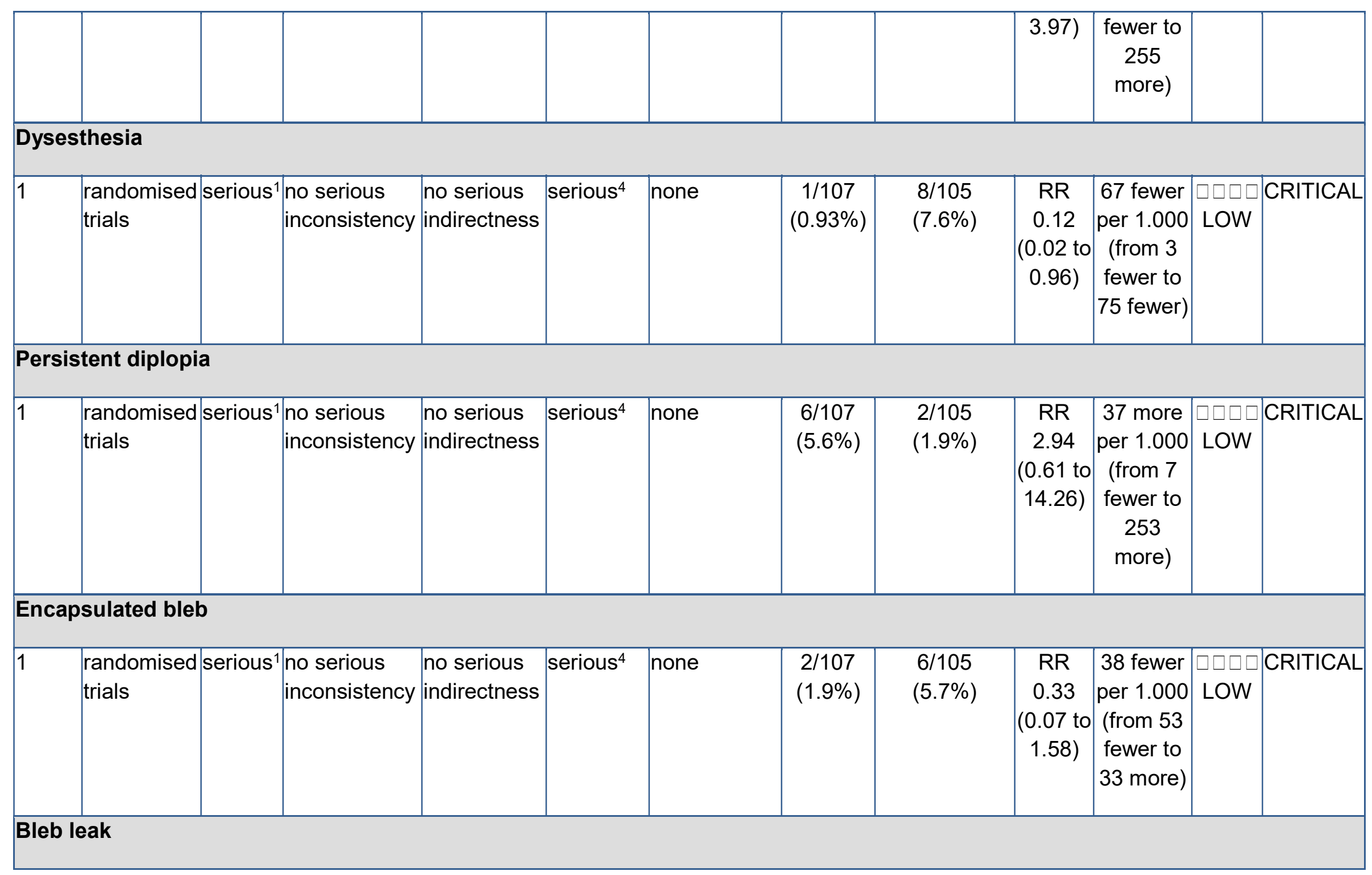




\begin{tabular}{|c|c|c|c|c|c|c|c|c|c|c|c|}
\hline 1 & $\mid \begin{array}{l}\text { randomised } \\
\text { trials }\end{array}$ & $\begin{array}{l}\text { no serious } \\
\text { inconsistency }\end{array}$ & $\begin{array}{l}\text { no serious } \\
\text { indirectness }\end{array}$ & serious ${ }^{4}$ & none & $\begin{array}{l}0 / 107 \\
(0 \%)\end{array}$ & $\begin{array}{l}6 / 105 \\
(5.7 \%)\end{array}$ & \begin{tabular}{|c|}
$\mathrm{RR}$ \\
$0.08(0$ \\
to 1.32$)$
\end{tabular} & \begin{tabular}{|l}
53 fewer \\
per 1.000 \\
(from 57 \\
fewer to \\
18 more)
\end{tabular} & $\begin{array}{l}\square \square \square \\
\text { LOW }\end{array}$ & CRITICAL \\
\hline \multicolumn{12}{|c|}{ Choroidal effusion } \\
\hline 1 & $\mid \begin{array}{l}\text { randomised } \\
\text { trials }\end{array}$ & $\begin{array}{l}\text { no serious } \\
\text { inconsistency }\end{array}$ & $\begin{array}{l}\text { no serious } \\
\text { indirectness }\end{array}$ & serious ${ }^{4}$ & none & $\begin{array}{l}2 / 107 \\
(1.9 \%)\end{array}$ & $\begin{array}{l}4 / 105 \\
(3.8 \%)\end{array}$ & \begin{tabular}{|c|} 
RR \\
0.49 \\
$(0.09$ to \\
$2.62)$
\end{tabular} & $\begin{array}{c}19 \text { fewer } \\
\text { per } 1.000 \\
\text { (from } 35 \\
\text { fewer to } \\
62 \text { more) }\end{array}$ & $\begin{array}{l}\text { पिमा } \\
\text { LOW }\end{array}$ & CRITICAL \\
\hline \multicolumn{12}{|c|}{ Cystoid macular edema } \\
\hline 1 & $\mid \begin{array}{l}\text { randomised } \\
\text { trials }\end{array}$ & $\begin{array}{l}1 \text { no serious } \\
\text { inconsistency }\end{array}$ & $\begin{array}{l}\text { no serious } \\
\text { indirectness }\end{array}$ & serious $^{4}$ & none & $\begin{array}{l}5 / 107 \\
(4.7 \%)\end{array}$ & $\begin{array}{c}2 / 105 \\
(1.9 \%)\end{array}$ & \begin{tabular}{|c|}
$R R$ \\
2.45 \\
$(0.49$ to \\
$12.37)$
\end{tabular} & \begin{tabular}{|c|}
28 more \\
per 1.000 \\
(from 10 \\
fewer to \\
217 \\
more)
\end{tabular} & $\begin{array}{l}\text { पिमा } \\
\text { LOW }\end{array}$ & CRITICAL \\
\hline \multicolumn{12}{|c|}{ Hypotony maculopathy } \\
\hline 1 & $\mid$\begin{tabular}{l|l} 
randomised \\
trials
\end{tabular} & $\begin{array}{l}\text { no serious } \\
\text { inconsistency }\end{array}$ & $\begin{array}{l}\text { no serious } \\
\text { indirectness }\end{array}$ & serious $^{4}$ & none & $\begin{array}{c}1 / 107 \\
(0.93 \%)\end{array}$ & $\begin{array}{l}5 / 105 \\
(4.8 \%)\end{array}$ & \begin{tabular}{|c}
$\mathrm{RR}$ \\
0.20 \\
$(0.02$ to \\
$1.65)$
\end{tabular} & $\begin{array}{c}38 \text { fewer } \\
\text { per } 1.000 \\
\text { (from } 47 \\
\text { fewer to } \\
31 \text { more) }\end{array}$ & LOW & CRITICAL \\
\hline
\end{tabular}

1 Did not specify methods of allocation concealment and it is not blinding 2 OAG progression as inferred from IOP.

${ }^{3}$ Small sample.

${ }^{4}$ Low number of events. $95 \% \mathrm{Cl}$ does not exclude appreciable benefit or harm. 


\begin{tabular}{|c|c|c|c|c|c|c|c|c|c|c|c|}
\hline & & & & & & & & 1.28) $f$ & \begin{tabular}{|c|}
107 \\
fewer to \\
251 \\
more)
\end{tabular} & & \\
\hline \multicolumn{12}{|c|}{ Qualified success rate (follow-up mean 24 months; assessed with: IOP $<21 \mathrm{mmHg}$ with medication) } \\
\hline 1 & 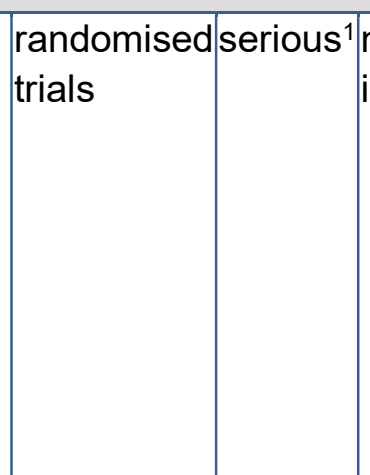 & \begin{tabular}{|l|}
$\mid$ no serious \\
inconsistency
\end{tabular} & serious $^{2}$ & serious $^{3}$ & none & $\begin{array}{c}1 / 19 \\
(5.3 \%)\end{array}$ & $\begin{array}{c}2 / 19 \\
(10.5 \%)\end{array}$ & \begin{tabular}{|c|}
$\mathrm{RR}$ \\
0.05 \\
$(0.05$ \\
to \\
$5.06)$ \\
\\
\end{tabular} & \begin{tabular}{|c|}
100 \\
fewer \\
per \\
1.000 \\
(from \\
100 \\
fewer to \\
427 \\
more)
\end{tabular} & \begin{tabular}{|l|} 
VED \\
VERY \\
LOW
\end{tabular} & CRITICAL \\
\hline \multicolumn{12}{|c|}{ Adverse event: symptomatic cataract (follow-up mean 24 months) } \\
\hline 1 & \begin{tabular}{|l|l|} 
randomised serious & 1 \\
trials
\end{tabular} & \begin{tabular}{|l|} 
no serious \\
inconsistency
\end{tabular} & \begin{tabular}{|l} 
no serious \\
indirectness
\end{tabular} & serious $^{3}$ & none & $\begin{array}{c}7 / 18 \\
(38.9 \%)\end{array}$ & $\begin{array}{c}8 / 17 \\
(47.1 \%)\end{array}$ & \begin{tabular}{|c|}
$\mathrm{RR}$ \\
0.83 \\
$(0.38$ \\
to \\
$1.78)$ \\
\end{tabular} & \begin{tabular}{|c|}
80 \\
fewer \\
per \\
1.000 \\
(from \\
292 \\
fewer to \\
367 \\
more)
\end{tabular} & \begin{tabular}{|l|} 
पि口प \\
LOW
\end{tabular} & CRITICAL \\
\hline
\end{tabular}

1 Open study: observers were not masked, the confunders (corneal astigmatism and refractive aberrations) were not analized. Sample size calculate in base of an indirect outcome (IOP). ITT analysis unclear.

OP considered as a surrogate outcome of visual field loss in patient with open angle glaucoma.

Wide $95 \%$ interval confidence and/or includes potential benefit or, potential harm of the intervention. 
4.5. Anestesia locoregional en la cirugía del glaucoma de ángulo abierto

4.5.1 Anestesia tópica respecto a anestesia retrobulbar

Question: Should Topical anaesthesia vs Retrobulbar anesthesia be used for open angle glaucoma surgery?

Bibliography:

Zabriskie NA, Ahmed II, Crandall AS, Daines B, Burns TA, Patel BC. A comparison of topical and retrobulbar anesthesia for trabeculectomy. J Glaucoma. 2002; 11(4): 306-14.

Sauder G, Jonas JB. Topical anesthesia for penetrating trabeculectomy. Graefes Arch Clin Exp Ophthalmol. 2002; 240(9): 739-42.

\begin{tabular}{|c|c|c|c|c|c|c|c|c|c|c|c|c|}
\hline \multicolumn{6}{|c|}{ Quality assessment } & \multicolumn{2}{|c|}{ No of patients } & \multicolumn{3}{|c|}{ Effect } & \multirow[t]{2}{*}{ Quality } & \multirow[t]{2}{*}{ Importance } \\
\hline $\begin{array}{c}\text { No of } \\
\text { studie } \\
\text { s }\end{array} \mid$ & Design & Risk of bias & $\begin{array}{c}\text { Inconsisten } \\
\text { cy }\end{array}$ & $\begin{array}{c}\text { Indirectnes } \\
\mathbf{s}\end{array}$ & $\begin{array}{c}\text { Imprecisio } \\
\mathrm{n}\end{array}$ & $\begin{array}{c}\text { Other } \\
\text { consideratio } \\
\text { ns }\end{array}$ & $\begin{array}{c}\text { Topical } \\
\text { anaesthesi } \\
\text { a }\end{array}$ & $\begin{array}{c}\text { Retrobulb } \\
\text { ar } \\
\text { anesthesi } \\
\text { a }\end{array}$ & $\begin{array}{c}\text { Relativ } \\
\text { e } \\
(95 \% \\
\mathrm{Cl})\end{array}$ & $\begin{array}{c}\text { Absolut } \\
\text { e }\end{array}$ & & \\
\hline
\end{tabular}

Pain during delivery anesthesia (Zabriski 2002) (follow-up 1 days; measured with: VAS 10 points; better indicated by lower values)

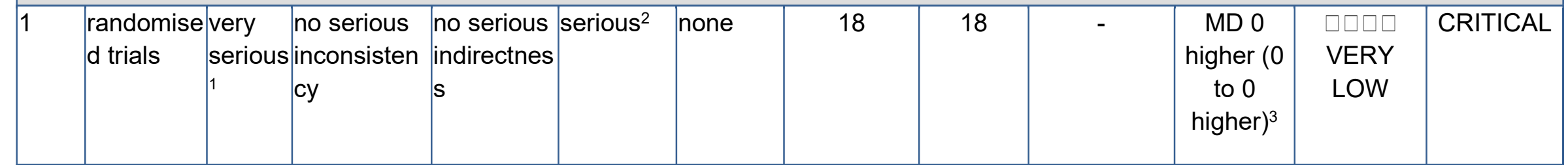

Pain during surgery (Sauder 2002) (follow-up 1 days; measured with: VAS 10 points; better indicated by lower values)

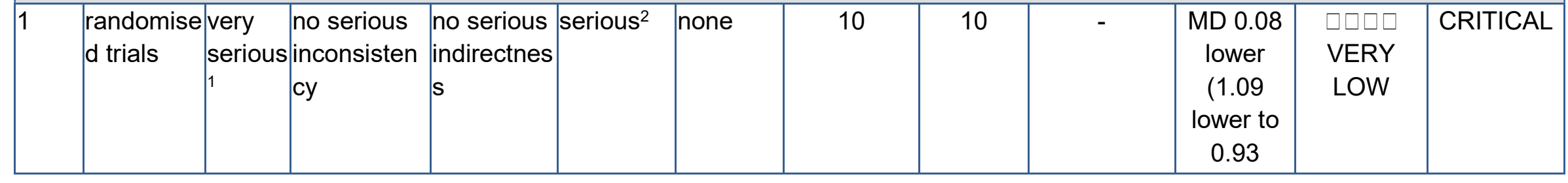




\begin{tabular}{|c|c|c|c|c|c|c|c|c|c|c|c|c|}
\hline & & & & & & & & & & higher) & & \\
\hline \multicolumn{13}{|c|}{ Pain during surgery (Zabriskie 2002) (follow-up 1 days; measured with: VAS 10 points; better indicated by lower values) } \\
\hline 1 & $\begin{array}{l}\text { randomise } \\
\mathrm{d} \text { trials }\end{array}$ & $\begin{array}{l}\text { very } \\
\text { serious } \\
1\end{array}$ & $\begin{array}{l}\text { no serious } \\
\text { inconsisten } \\
\text { cy }\end{array}$ & $\begin{array}{l}\mid \begin{array}{l}\text { no serious } \\
\text { indirectnes } \\
\mathrm{s}\end{array} \\
\end{array}$ & serious $^{2}$ & none & 18 & 18 & - & $\begin{array}{c}\text { MD 0 } \\
\text { higher (0 } \\
\text { to } 0 \\
\text { higher })^{4}\end{array}$ & $\begin{array}{l}\text { पिमा } \\
\text { VERY } \\
\text { LOW }\end{array}$ & CRITICAL \\
\hline \multicolumn{13}{|c|}{ Pain after surgery (Zabriskie 2002) (follow-up 1 days; measured with: VAS 10 points; better indicated by lower values) } \\
\hline 1 & $\begin{array}{l}\text { randomise } \\
\text { d trials }\end{array}$ & \begin{tabular}{|l} 
very \\
serious \\
1
\end{tabular} & $\begin{array}{l}\text { no serious } \\
\text { inconsisten } \\
\text { cy }\end{array}$ & $\begin{array}{l}\mid \begin{array}{l}\text { no serious } \\
\text { indirectnes } \\
\text { s }\end{array} \\
\end{array}$ & serious $^{2}$ & none & 18 & 18 & - & \begin{tabular}{|c|} 
MD 0 \\
higher (0 \\
to 0 \\
higher $)^{5}$
\end{tabular} & $\begin{array}{l}\text { पिमा } \\
\text { VERY } \\
\text { LOW }\end{array}$ & CRITICAL \\
\hline \multicolumn{13}{|c|}{ Additional anaesthesia (assessed with: number of patients requiring additional anaesthesia) } \\
\hline 1 & $\begin{array}{l}\text { randomise } \\
\text { d trials }\end{array}$ & $\begin{array}{l}\text { serious } \\
1\end{array}$ & $\begin{array}{l}\text { no serious } \\
\text { inconsisten } \\
\text { cy }\end{array}$ & $\begin{array}{l}\text { no serious } \\
\text { indirectnes } \\
\mathrm{s}\end{array}$ & serious $^{2}$ & $\begin{array}{l}\text { reporting } \\
\text { bias }^{6}\end{array}$ & - & - & - & - & $\begin{array}{l}\text { पिएव } \\
\text { VERY } \\
\text { LOW }\end{array}$ & CRITICAL \\
\hline \multicolumn{13}{|c|}{ Subconjunctival hemorrhage (Zabriskie 2002) } \\
\hline 1 & $\begin{array}{l}\text { randomise } \\
\mathrm{d} \text { trials }\end{array}$ & \begin{tabular}{|l} 
very \\
serious \\
1
\end{tabular} & $\begin{array}{l}\text { no serious } \\
\text { inconsisten } \\
\text { cy }\end{array}$ & $\begin{array}{l}\mid \begin{array}{l}\text { no serious } \\
\text { indirectnes } \\
\text { s }\end{array} \\
\end{array}$ & serious $^{2}$ & none & $\begin{array}{l}0 / 18 \\
(0 \%)\end{array}$ & \begin{tabular}{|c|}
$6 / 18$ \\
$(33.3 \%)$
\end{tabular} & $\begin{array}{c}\text { RR } 0.08(0 \\
\text { to } 1.27)\end{array}$ & $\begin{array}{c}307 \text { fewer } \\
\text { per } 1.000 \\
\text { (from } 333 \\
\text { fewer to } \\
90 \text { more) }\end{array}$ & $\begin{array}{l}\text { पिमा } \\
\text { VERY } \\
\text { LOW }\end{array}$ & CRITICAL \\
\hline \multicolumn{13}{|c|}{ Chemosis (Zabriskie 2002) } \\
\hline 1 & $\begin{array}{l}\text { randomise } \\
\mathrm{d} \text { trials }\end{array}$ & $\begin{array}{l}\text { very } \\
\text { serious } \\
1\end{array}$ & $\begin{array}{l}\text { no serious } \\
\text { inconsisten } \\
\text { cy }\end{array}$ & $\begin{array}{l}\mid \begin{array}{l}\text { no serious } \\
\text { indirectnes } \\
\text { s }\end{array} \\
\end{array}$ & serious $^{2}$ & none & $\begin{array}{l}0 / 18 \\
(0 \%)\end{array}$ & \begin{tabular}{|c|}
$12 / 18$ \\
$(66.7 \%)$
\end{tabular} & $\begin{array}{c}\text { RR } 0.04(0 \\
\text { to } 0.63)\end{array}$ & $\begin{array}{c}640 \text { fewer } \\
\text { per } 1.000 \\
\text { (from } 247 \\
\text { fewer to }\end{array}$ & $\begin{array}{l}\text { पिमा } \\
\text { VERY } \\
\text { LOW }\end{array}$ & CRITICAL \\
\hline
\end{tabular}




\begin{tabular}{|c|c|c|c|c|c|c|c|c|c|c|c|c|}
\hline & & & & & & & & & & $\begin{array}{c}667 \\
\text { fewer) }\end{array}$ & & \\
\hline \multicolumn{13}{|c|}{ Hematoma parpebral (Zabriskie 2002) } \\
\hline 1 & $\begin{array}{l}\text { randomise } \\
\text { d trials }\end{array}$ & \begin{tabular}{|l|} 
very \\
serious \\
1
\end{tabular} & $\begin{array}{l}\text { no serious } \\
\text { sinconsisten } \\
\text { cy }\end{array}$ & $\begin{array}{l}\text { no serious } \\
\text { indirectnes } \\
\text { s }\end{array}$ & & none & $\begin{array}{l}0 / 18 \\
(0 \%)\end{array}$ & $\begin{array}{c}10 / 18 \\
(55.6 \%)\end{array}$ & $\begin{array}{c}\text { RR } 0.05(0) \\
\text { to } 0.76)\end{array}$ & \begin{tabular}{|c|}
528 fewer \\
per 1.000 \\
(from 133 \\
fewer to \\
556 \\
fewer)
\end{tabular} & & \begin{tabular}{|c|} 
IMPORTAN \\
$T$
\end{tabular} \\
\hline \multicolumn{13}{|c|}{ Pain during delivery anesthesia- Ahmed implant (better indicated by lower values) } \\
\hline 1 & $\begin{array}{l}\text { randomise } \\
\text { d trials }\end{array}$ & serious & \begin{tabular}{|l|} 
sno serious \\
inconsisten \\
cy
\end{tabular} & $\begin{array}{l}\text { no serious } \\
\text { indirectnes } \\
\text { s }\end{array}$ & \begin{tabular}{|l|} 
no \\
serious \\
imprecisio \\
n
\end{tabular} & none & 16 & 16 & - & \begin{tabular}{|c|} 
MD 2.62 \\
lower \\
$(3.98$ to \\
1.26 \\
lower)
\end{tabular} & $\begin{array}{c}\text { MODERAT } \\
\mathrm{E}\end{array}$ & CRITICAL \\
\hline \multicolumn{13}{|c|}{ Pain during surgery- Ahmed implant (better indicated by lower values) } \\
\hline 1 & $\mid \begin{array}{l}\text { randomise } \\
\text { d trials }\end{array}$ & serious & $\begin{array}{l}\text { no serious } \\
\text { inconsisten } \\
\text { cy }\end{array}$ & $\begin{array}{l}\text { no serious } \\
\text { indirectnes } \\
\text { s }\end{array}$ & serious $^{2}$ & none & 16 & 16 & - & \begin{tabular}{|c|} 
MD 0.62 \\
higher \\
$(0.45$ \\
lower to \\
1.69 \\
higher)
\end{tabular} & $\begin{array}{l}\text { पिएव } \\
\text { LOW }\end{array}$ & CRITICAL \\
\hline \multicolumn{13}{|c|}{ Pain after surgery- Ahmed implant (better indicated by lower values) } \\
\hline 1 & $\begin{array}{l}\text { randomise } \\
\text { d trials }\end{array}$ & serious & $\begin{array}{l}\text { no serious } \\
\text { inconsisten } \\
\text { cy }\end{array}$ & $\begin{array}{l}\text { no serious } \\
\text { indirectnes } \\
\text { s }\end{array}$ & serious $^{2}$ & none & 16 & 16 & - & \begin{tabular}{|c|} 
MD 0.21 \\
higher \\
$(0.82$ \\
lower to \\
1.24
\end{tabular} & $\begin{array}{l}\text { पिएव } \\
\text { LOW }\end{array}$ & CRITICAL \\
\hline
\end{tabular}




\begin{tabular}{|l|l|l|l|l|l|l|l|l|l|l|l|}
\hline & & & & & & higher) & & \\
\hline
\end{tabular}

1 High risk of performance and detection bias/attrition bias unclear

2 Interval confidence $95 \%$ include potential benefit and potential harm/low number of events.

VAS mean score Topical group $=0,22 ;$ VAS mean score retrobulbar 0,$06 ; p=0,15$.. Standard deviation not reported.

VAS mean score topical group 0,5 ; VAS mean score retrobulbar group 0,39. $p=0,54$. Standard deviation not reported.

5 VAS mean score topical group 0,44 ; VAS mean score retrobulbar group $0,39 . p=0,76$. Standard deviation not reported.

Number of events with supplementary anaesthesia where not reported or only reported for one group. 


\section{MÉTODOS}

Como medida del efecto, para las variables dicotómicas se utilizó el cociente de riesgos (CR) y para las variables continuas se usó la diferencia de medias ponderadas (DMP), ambos con sus intervalos de confianza del 95\%. Como método estadístico se utilizaron,

respectivamente, los métodos de Mantel-Haenszel y de la varianza inversa. Para el metanálisis se utilizó el modelo de efectos fijos excepto cuando se encontró heterogeneidad significativa; en ese caso, se utilizó el modelo de efectos aleatorios.

FOREST PLOTS

\section{Anestesia tópica vs. retrobulbar. Dolor durante la cirugía}

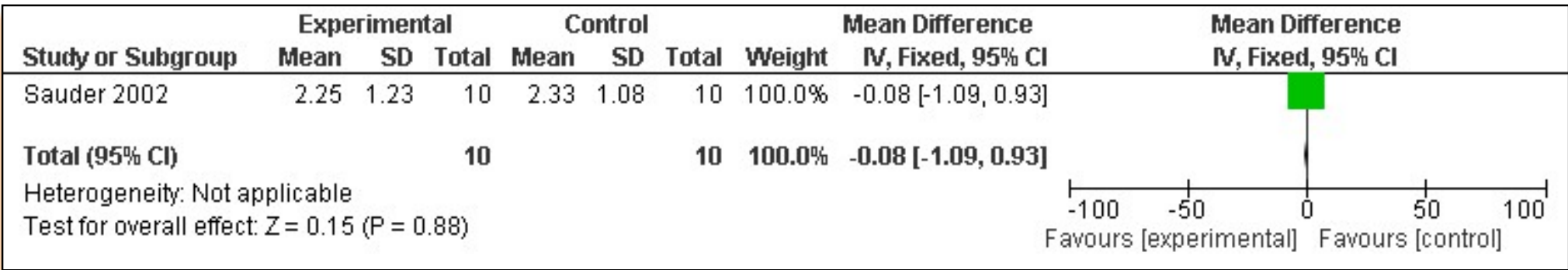

\section{Anestesia tópica vs. retrobulbar. Hemorragia subconjuntival}

\begin{tabular}{|c|c|c|c|c|c|c|c|c|}
\hline \multirow[b]{2}{*}{ Study or Subgroup } & \multicolumn{2}{|c|}{ Experimental } & \multicolumn{2}{|c|}{ Control } & \multirow[b]{2}{*}{ Weight } & \multirow{2}{*}{$\begin{array}{c}\text { Risk Ratio } \\
\text { M-H, Fixed, 95\% Cl }\end{array}$} & \multirow{2}{*}{\multicolumn{2}{|c|}{$\begin{array}{c}\text { Risk Ratio } \\
\text { M-H, Fixed, 95\% Cl }\end{array}$}} \\
\hline & Events & Total & Events & Total & & & & \\
\hline Zabriskie & 0 & 18 & 6 & 18 & $100.0 \%$ & $0.08[0.00,1.27]$ & 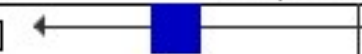 & \\
\hline Total $(95 \% \mathrm{Cl})$ & & 18 & & 18 & $100.0 \%$ & $0.08[0.00,1.27]$ & & \\
\hline Total events & 0 & & 6 & & & & & \\
\hline $\begin{array}{l}\text { Heterogeneity: Not a } \\
\text { Test for overall effect }\end{array}$ & $\begin{array}{l}\text { plicable } \\
Z=1.79(\end{array}$ & $=0.07$ & & & & & \begin{tabular}{cc|}
0.01 & 0.1 \\
Favours [experimentall]
\end{tabular} & $\begin{array}{c}10 \\
100 \\
\text { Favours [control] }\end{array}$ \\
\hline
\end{tabular}




\section{Anestesia tópica vs. retrobulbar.Quemosis}

\begin{tabular}{|c|c|c|c|c|c|c|c|c|c|}
\hline \multirow[b]{2}{*}{ Study or Subgroup } & \multicolumn{2}{|c|}{ Experimental } & \multicolumn{2}{|c|}{ Control } & \multicolumn{2}{|r|}{ Risk Ratio } & \multirow{2}{*}{\multicolumn{3}{|c|}{$\begin{array}{c}\text { Risk Ratio } \\
\text { N, Fixed, 95\% Cl }\end{array}$}} \\
\hline & Events & Total & Events & Total & Weight & 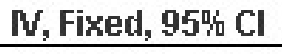 & & & \\
\hline Zabriskie & 0 & 18 & 12 & 18 & $100.0 \%$ & $0.04[0.00,0.63]$ & & & \\
\hline Total $(95 \%$ Cl) & & 18 & & 18 & $100.0 \%$ & $0.04[0.00,0.63]$ & & & \\
\hline Total events & 0 & & 12 & & & & & & \\
\hline $\begin{array}{l}\text { Heterogeneity: Not a } \\
\text { Test for overall effec }\end{array}$ & $\begin{array}{l}\text { plicable } \\
Z=2.29\end{array}$ & $=0.02$ & & & & & $\begin{array}{rc} & 0.01 \\
= & 0.1 \\
\text { avours [experimental] }\end{array}$ & 1] Favours & $\begin{array}{l}10{ }^{1} 100 \\
\text { [control] }\end{array}$ \\
\hline
\end{tabular}

\section{Anestesia tópica vs. retrobulbar. Hematoma parpebral}

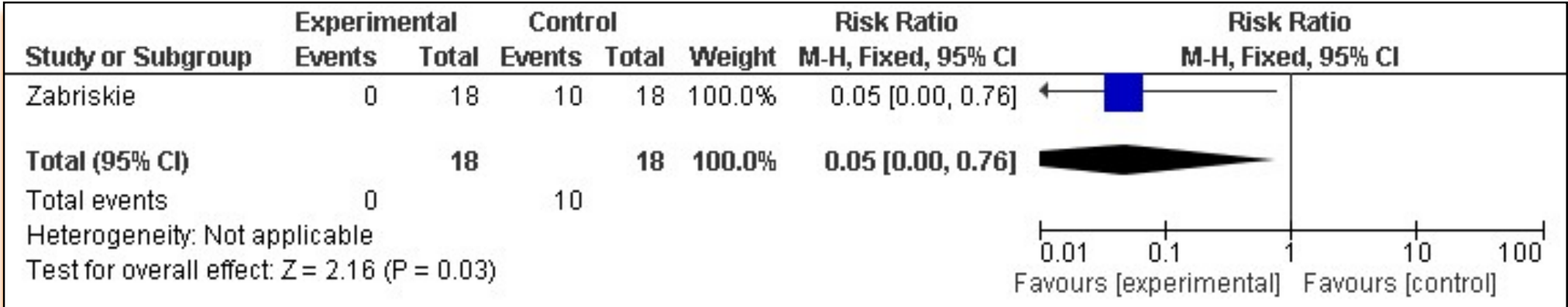




\subsection{Anestesia tópica respecto a anestesia subtenoniana}

Question: Should Topical anaesthesia vs sub-Tenon's anaesthesia be used for open angle glaucoma surgery?

\section{Bibliography:}

Carrillo MM, Buys YM, Faingold D, Trope GE. Prospective study comparing lidocaine $2 \%$ jelly versus sub-Tenon's anaesthesia for trabeculectomy surgery. Br J Ophthalmol. 2004; 88(8): 1004-7.

\begin{tabular}{|c|c|c|c|c|c|c|c|c|c|c|c|c|}
\hline \multicolumn{7}{|c|}{ Quality assessment } & \multicolumn{2}{|c|}{ No of patients } & \multicolumn{2}{|c|}{ Effect } & \multirow[t]{2}{*}{ Quality } & \multirow[t]{2}{*}{ Importance } \\
\hline $\begin{array}{c}\text { No of } \\
\text { studies }\end{array}$ & Design & Risk of bias & Inconsistency & Indirectness & Imprecision & $\begin{array}{c}\text { Other } \\
\text { considerations }\end{array}$ & $\begin{array}{c}\text { Topical } \\
\text { anaesthesia }\end{array}$ & $\begin{array}{c}\text { Sub- } \\
\text { Tenon's } \\
\text { anaesthesia }\end{array}$ & $\begin{array}{l}\text { Relative } \\
(95 \% \mathrm{Cl})\end{array}$ & l) Absolute & & \\
\hline \multicolumn{13}{|c|}{ Pain during surgery (better indicated by lower values) } \\
\hline 1 & $\begin{array}{l}\text { randomised } \\
\text { trials }\end{array}$ & $\begin{array}{l}\text { very } \\
\text { serious }{ }^{1}\end{array}$ & $\begin{array}{l}\text { no serious } \\
\text { inconsistency }\end{array}$ & $\begin{array}{l}\text { no serious } \\
\text { indirectness }\end{array}$ & serious $^{2}$ & none & 30 & 29 & I & $\begin{array}{c}\text { MD 1.00 } \\
\text { higher } \\
(6.38 \\
\text { lower to } \\
8.37 \\
\text { higher) }\end{array}$ & $\begin{array}{l}\text { पिएव } \\
\text { VERY } \\
\text { LOW }\end{array}$ & CRITICAL \\
\hline \multicolumn{13}{|c|}{ Adverse events } \\
\hline 1 & $\begin{array}{l}\text { randomised } \\
\text { trials }\end{array}$ & $\begin{array}{l}\text { very } \\
\text { serious }{ }^{1}\end{array}$ & \begin{tabular}{|l|} 
no serious \\
inconsistency
\end{tabular} & $\begin{array}{l}\text { no serious } \\
\text { indirectness }\end{array}$ & \begin{tabular}{|l|} 
no serious \\
imprecision
\end{tabular} & none & $\begin{array}{l}0 / 30 \\
(0 \%)\end{array}$ & $\begin{array}{l}0 / 29 \\
(0 \%)\end{array}$ & $\begin{array}{c}\text { not } \\
\text { pooled }\end{array}$ & \begin{tabular}{c|} 
not \\
pooled
\end{tabular} & $\begin{array}{l}\text { पिमा } \\
\text { LOW }\end{array}$ & \\
\hline \multicolumn{13}{|c|}{ Probability of complete success } \\
\hline 1 & $\begin{array}{l}\text { observational } \\
\text { studies }\end{array}$ & $\begin{array}{l}\text { no serious } \\
\text { risk of bias }\end{array}$ & $\left|\begin{array}{l}\text { no serious } \\
\text { inconsistency }\end{array}\right|$ & \begin{tabular}{|l|} 
no serious \\
indirectness
\end{tabular} & \begin{tabular}{|l|} 
no serious \\
imprecision
\end{tabular} & none & - & - & - & - & $\begin{array}{l}\text { पिमा } \\
\text { LOW }\end{array}$ & CRITICAL \\
\hline
\end{tabular}




\begin{tabular}{|c|c|c|c|c|c|c|c|c|c|c|c|c|}
\hline & $\begin{array}{l}\text { observationa } \\
\text { studies }\end{array}$ & $\begin{array}{l}\text { nno serious } \\
\text { risk of bias }\end{array}$ & \begin{tabular}{|l} 
no serious \\
inconsistency
\end{tabular} & \begin{tabular}{|l|} 
no serious \\
indirectness
\end{tabular} & \begin{tabular}{|l|} 
no serious \\
imprecision
\end{tabular} & none & - & - & \begin{tabular}{c|} 
not \\
pooled
\end{tabular} & $\begin{array}{c}\text { not } \\
\text { pooled }\end{array}$ & $\begin{array}{l}\text { पिए। } \\
\text { LOW }\end{array}$ & CRITICAL \\
\hline & \multicolumn{12}{|c|}{ Additional anesthesia } \\
\hline & $\begin{array}{l}\text { randomised } \\
\text { trials }\end{array}$ & serious $^{1}$ & \begin{tabular}{|l} 
no serious \\
inconsistency
\end{tabular} & \begin{tabular}{|l|} 
no serious \\
indirectness
\end{tabular} & \begin{tabular}{|l} 
very \\
serious $^{3}$
\end{tabular} & none & $\begin{array}{l}0 / 30 \\
(0 \%)\end{array}$ & $\begin{array}{c}4 / 29 \\
(13.8 \%)\end{array}$ & \begin{tabular}{|c|}
$\mathrm{RR}$ \\
0.11 \\
$(0.01$ \\
to \\
$1.91)$
\end{tabular} & \begin{tabular}{|c}
123 \\
fewer \\
per \\
1.000 \\
(from \\
137 \\
fewer to \\
126 \\
more)
\end{tabular} & $\begin{array}{l}\text { पिमा } \\
\text { VERY } \\
\text { LOW }\end{array}$ & CRITICAL \\
\hline
\end{tabular}

${ }^{1}$ High risk of performance and detection bias/attrition bias unclear

${ }_{2}$ Interval confidence $95 \%$ include potential benefit and potential harm/low number of events.

${ }^{3}$ No explanation was provided. 


\section{MÉTODOS}

Como medida del efecto, para las variables dicotómicas se utilizó el cociente de riesgos (CR) y para las variables continuas se usó la diferencia de medias ponderadas (DMP), ambos con sus intervalos de confianza del 95\%. Como método estadístico se utilizaron, respectivamente, los métodos de Mantel-Haenszel y de la varianza inversa. Para el metanálisis se utilizó el modelo de efectos fijos, excepto cuando se encontró heterogeneidad significativa; en ese caso, se utilizó el modelo de efectos aleatorios.

\section{FOREST PLOTS}

\section{Dolor durante la cirugía}

\begin{tabular}{|c|c|c|c|c|c|c|c|c|c|}
\hline \multirow[b]{2}{*}{ Study or Subgroup } & \multicolumn{3}{|c|}{ Experimental } & \multicolumn{3}{|c|}{ Control } & \multicolumn{2}{|r|}{ Mean Difference } & \multirow{2}{*}{$\begin{array}{l}\text { Mean Difference } \\
\mathrm{N}, \text { Fixed, } 95 \% \mathrm{Cl}\end{array}$} \\
\hline & Mean & SD & Total & Mean & SD & Total & Weight & $\mathrm{N}$, Fixed, $95^{\circ} \mathrm{Cl}$ & \\
\hline Carillo 2004 & 19.3 & 12.4 & 30 & 18.3 & 16.2 & 29 & $100.0 \%$ & $1.00[-6.38,8.38]$ & \\
\hline Total $\left(95^{\circ} \mathrm{Cl}\right)$ & & & 30 & & & 29 & $100.0 \%$ & $1.00[-6.38,8.38]$ & \\
\hline $\begin{array}{l}\text { Heterogeneity: Not } \\
\text { Test for overall effec }\end{array}$ & $\begin{array}{l}\text { plicable } \\
Z=0.27\end{array}$ & & & & & & & & 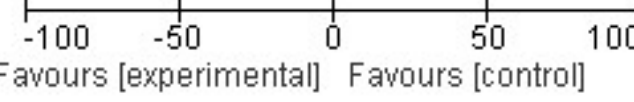 \\
\hline
\end{tabular}

\section{Uso adicional de anestesia durante la cirugía}

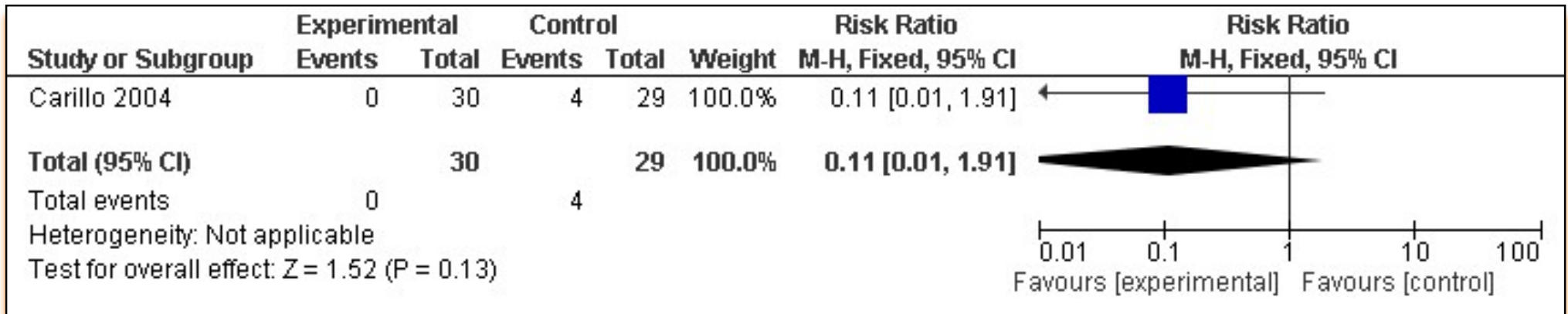


4.5.3 Anestesia tópica respecto a anestesia peribulbar

Question: Should Topic anaesthesia vs Peribulbar be used for open angle glaucoma surgery?

Bibliography:

Paletta RA, Paletta V, Pereira da silva A, Montezi F, Montezi P, Chaoubah. Anesthésie topique versus anesthésie péribulbaire dans la sclérectomie profonde non perforante. J Fr. Ophtalmol. 2007; 30(8): 825-829.

\begin{tabular}{|c|c|c|c|c|c|c|c|c|c|c|c|c|}
\hline \multicolumn{7}{|c|}{ Quality assessment } & \multicolumn{2}{|c|}{ No of patients } & \multicolumn{2}{|r|}{ Effect } & \multirow{2}{*}{ Quality } & \multirow{2}{*}{ Importance } \\
\hline $\begin{array}{l}\text { No of } \\
\text { studies }\end{array}$ & Design & $\begin{array}{c}\text { Risk of } \\
\text { bias }\end{array}$ & Inconsistency & Indirectness & Imprecision & \begin{tabular}{|c|} 
Other \\
considerations
\end{tabular} & \begin{tabular}{c|} 
Topic \\
anaesthesia
\end{tabular} & Peribulbar & \begin{tabular}{|c|} 
Relative \\
$(95 \%$ \\
Cl)
\end{tabular} & Absolute & & \\
\hline \multicolumn{13}{|c|}{ Pain 15 after surgery (measured with: VAs 10 points; better indicated by lower values) } \\
\hline 1 & $\begin{array}{l}\text { randomised } \\
\text { trials }\end{array}$ & $\begin{array}{l}\text { very } \\
\text { serious }\end{array}$ & $\begin{array}{l}\text { no serious } \\
\text { inconsistency }\end{array}$ & $\begin{array}{l}\text { no serious } \\
\text { indirectness }\end{array}$ & serious $^{2}$ & none & 36 & 33 & - & \begin{tabular}{|c|} 
MD 0.71 \\
lower $(1.23$ \\
lower to \\
0.19 \\
higher)
\end{tabular} & \begin{tabular}{|l|} 
पिएव \\
VERY \\
LOW
\end{tabular} & CRITICAL \\
\hline \multicolumn{13}{|c|}{ Pain 24 hours after surgery (better indicated by lower values) } \\
\hline 1 & \begin{tabular}{|l} 
randomised \\
trials
\end{tabular} & $\begin{array}{l}\text { very } \\
\text { serious }\end{array}$ & \begin{tabular}{|l|l} 
no serious \\
inconsistency
\end{tabular} & \begin{tabular}{|l|} 
no serious \\
indirectness
\end{tabular} & serious $^{2}$ & none & 36 & 33 & 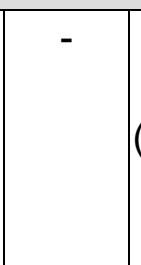 & \begin{tabular}{|c|} 
MD 0.38 \\
higher \\
(0.46 lower \\
to 1.22 \\
higher $)$
\end{tabular} & \begin{tabular}{|l|} 
पिएव \\
VERY \\
LOW
\end{tabular} & \\
\hline
\end{tabular}

${ }^{1}$ High risk of performance and detection bias. Selection and attrition bias unclear.

${ }^{2}$ Interval confidence $95 \%$ include potential benefit and potential harm. 


\section{MÉTODOS}

Como medida del efecto, para las variables dicotómicas se utilizó el cociente de riesgos (CR) y para las variables continuas se usó la diferencia de medias ponderadas (DMP), ambos con sus intervalos de confianza del 95\%. Como método estadístico se utilizaron, respectivamente, los métodos de Mantel-Haenszel y de la varianza inversa. Para el metanálisis se utilizó el modelo de efectos fijos, excepto cuando se encontró heterogeneidad significativa; en ese caso, se utilizó el modelo de efectos aleatorios.

\section{FOREST PLOTS}

\section{Anestesia tópica vs. anestesia peribulbar. Dolor 15 minutos después de la cirugía}

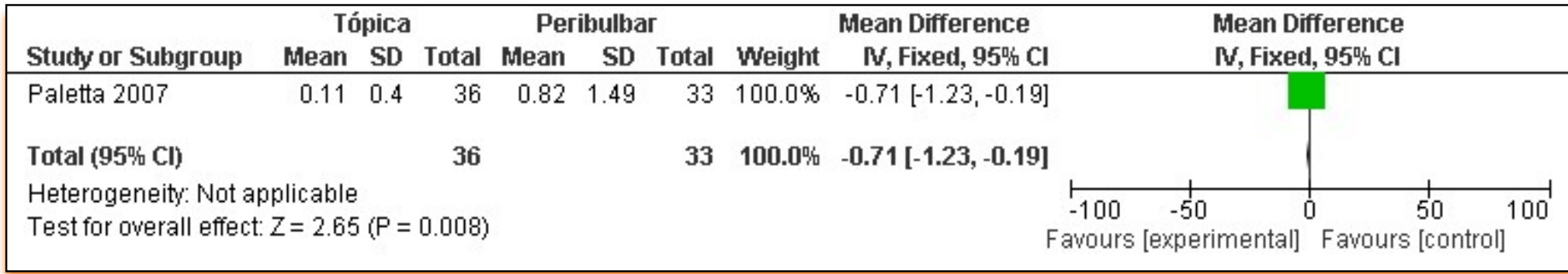

\section{Anestesia tópica vs. anestesia peribulbar. Dolor 24 horas después de la cirugía}

\begin{tabular}{|c|c|c|c|c|c|c|c|c|c|}
\hline \multirow[b]{2}{*}{ Stucty or Subgroup } & \multicolumn{3}{|c|}{ Tópica } & \multicolumn{3}{|c|}{ Peribulbar } & \multicolumn{2}{|r|}{ Mean Difference } & \multirow{2}{*}{$\begin{array}{l}\text { Mean Difference } \\
\mathrm{N}, \text { Fixed, } 95 \% \mathrm{Cl}\end{array}$} \\
\hline & Mean & SD & Total & Mean & SD & Total & Weight & $\mathrm{N}$, Fixed, 95听 $\mathrm{Cl}$ & \\
\hline Paletta 2007 & 2.83 & 1.34 & 36 & 2.45 & 2.09 & 33 & $100.0 \%$ & $0.38[-0.46,1.22]$ & \\
\hline Total (95\% Cl) & & & 36 & & & 33 & $100.0 \%$ & $0.38[-0.46,1.22]$ & \\
\hline $\begin{array}{l}\text { Heterogeneity: Not } \\
\text { Test for overall effec }\end{array}$ & $\begin{array}{l}\text { plicable } \\
Z=0.89\end{array}$ & & & & & & & & $\begin{array}{ccccc}-100 & -50 & 0 & 50 & 100 \\
\text { Favours [experimental] } & \text { Favours [control] }\end{array}$ \\
\hline
\end{tabular}


4.5.4 Anestesia de contacto vs. otras tecnicas anestésicas

Question: Should contac anaesthesia vs peribulbar anaesthesia be used for trabeculectomy?

Bibliography:

Pablo LE, Pérez-Oliván S, Ferreras A, Polo V, Gómez ML, Honrubia FM. Contact versus peribulbar anaesthesia in trabeculectomy: a prospective randomized clinical study. Acta Ophthalmol Scand. 2003 Oct; 81(5): 486-90.

\begin{tabular}{|c|c|c|c|c|c|c|c|c|c|c|c|c|}
\hline \multicolumn{7}{|c|}{ Quality assessment } & \multicolumn{2}{|c|}{ No of patients } & \multicolumn{2}{|c|}{ Effect } & \multirow[t]{2}{*}{ Quality } & \multirow[t]{2}{*}{ Importance } \\
\hline $\begin{array}{l}\text { No of } \\
\text { studies }\end{array}$ & Design & $\begin{array}{c}\text { Risk of } \\
\text { bias }\end{array}$ & Inconsistency & Indirectness & Imprecision & $\begin{array}{c}\text { Other } \\
\text { considerations }\end{array}$ & $\begin{array}{c}\text { Contac } \\
\text { anaesthesia }\end{array}$ & $\begin{array}{c}\text { Peribulbar } \\
\text { anaesthesia }\end{array}$ & $\begin{array}{c}\text { Relative } \\
(95 \% \\
\text { CI) }\end{array}$ & Absolute & & \\
\hline \multicolumn{13}{|c|}{ Intraocular pressure (follow-up 3 months; better indicated by lower values) } \\
\hline 1 & $\begin{array}{l}\text { randomise } \\
\text { trials }\end{array}$ & $\begin{array}{l}\text { d very } \\
\text { serious }^{1}\end{array}$ & $\begin{array}{l}\text { no serious } \\
\text { 1inconsistency }\end{array}$ & $\begin{array}{l}\text { no serious } \\
\text { yndirectness }\end{array}$ & serious $^{2}$ & none & 50 & 50 & - & $\begin{array}{c}\text { MD 0.4 } \\
\text { higher } \\
(1.08 \\
\text { lower to } \\
1.88 \\
\text { higher }\end{array}$ & $\begin{array}{l}\text { पिमा } \\
\text { VERY } \\
\text { LOW }\end{array}$ & CRITICAL \\
\hline
\end{tabular}

Pain during the administration of the anaesthetic agent (follow-up 1 days; measured with: 5-point scale; ${ }^{3}$ better indicated by lower values)

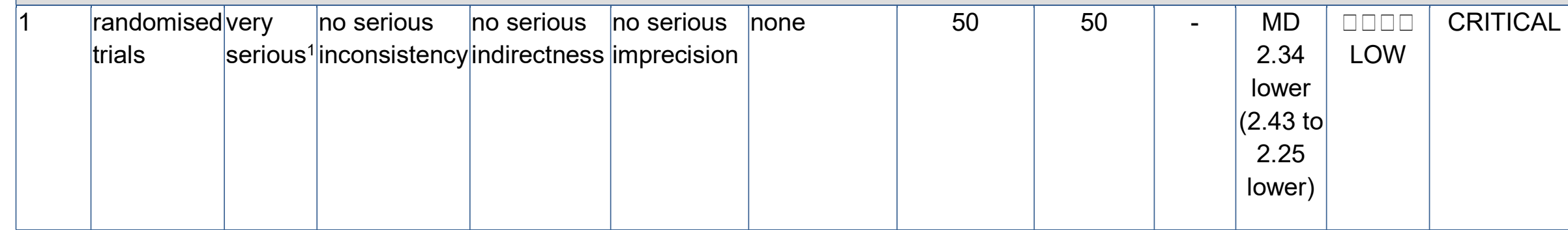

Subjetive surgeon stress assessment (follow-up 3 months; measured with: 4-point scale; better indicated by lower values) 


\begin{tabular}{|c|c|c|c|c|c|c|c|c|c|c|c|}
\hline 1 & $\begin{array}{l}\text { randomised serious } 1 \\
\text { trials }\end{array}$ & $\begin{array}{l}1 \text { no serious } \\
\text { inconsistency }\end{array}$ & \begin{tabular}{|l} 
no serious \\
yindirectness
\end{tabular} & serious $^{2}$ & none & 50 & 50 & - & \begin{tabular}{|c|}
$\mathrm{MD}$ \\
0.06 \\
higher \\
$(0.1$ \\
lower to \\
0.22 \\
higher $)$
\end{tabular} & $\begin{array}{l}\text { पिमा } \\
\text { LOW }\end{array}$ & IMPORTANT \\
\hline \multicolumn{12}{|c|}{ Chemosis/conjuntival haemorrage (follow-up 1 months) } \\
\hline 1 & $\begin{array}{l}\text { randomised serious } 1 \\
\text { trials }\end{array}$ & $\begin{array}{l}\text { no serious } \\
\text { inconsistency }\end{array}$ & $\begin{array}{l}\text { no serious } \\
\text { indirectness }\end{array}$ & serious $^{2}$ & none & $\begin{array}{l}0 / 50 \\
(0 \%)\end{array}$ & $\begin{array}{l}6 / 50 \\
(12 \%)\end{array}$ & \begin{tabular}{|c|} 
RR \\
$0.07(0$ \\
to \\
$1.24)$
\end{tabular} & \begin{tabular}{|c|}
112 \\
fewer \\
per \\
1.000 \\
(from \\
120 \\
fewer \\
to 29 \\
more)
\end{tabular} & $\begin{array}{l}\text { पिपा } \\
\text { LOW }\end{array}$ & CRITICAL \\
\hline \multicolumn{12}{|c|}{ Total complications (follow-up 1 months) } \\
\hline 1 & $\begin{array}{l}\text { randomised serious }{ }^{1} \\
\text { trials }\end{array}$ & $\begin{array}{l}1 \text { no serious } \\
\text { inconsistency }\end{array}$ & $\begin{array}{l}\text { no serious } \\
\text { yindirectness }\end{array}$ & serious $^{2}$ & none & $\begin{array}{l}11 / 50 \\
(22 \%)\end{array}$ & $\begin{array}{l}11 / 50 \\
(22 \%)\end{array}$ & \begin{tabular}{|c|}
$R R$ \\
1.00 \\
$(0.39$ \\
to \\
$2.58)$
\end{tabular} & $\begin{array}{c}0 \text { fewer } \\
\text { per } \\
1.000 \\
\text { (from } \\
134 \\
\text { fewer } \\
\text { to } 348 \\
\text { more) }\end{array}$ & $\begin{array}{l}\text { LOप } \\
\text { LOW }\end{array}$ & CRITICAL \\
\hline
\end{tabular}

${ }^{1}$ High risk of performance bias, detection bias unclear. The study use not standardized pain scale with different cathegories using different categories. Data analysis: the variable is analyzed as continuous one.

Interval confidence $95 \%$ include potential benefit and potential harm/low number of events. 
${ }^{3} 0=$ no pain, $1=$ mild discomfort, $2=$ mild pain, $3=$ moderate pain, 4 = severe pain

Question: Should contact anaesthesia vs topica anaesthesia be used for trabeculectomy?

Bibliography:

Pablo LE, Pérez-Oliván S, Ferreras A, Larrosa JM, Gómez ML, Honrubia FM.Topical versus contact anaesthesia in conventional trabeculectomy. Prospective randomised study.Arch Soc Esp Oftalmol. 2003; 75(5): 251-256.

\begin{tabular}{|c|c|c|c|c|c|c|c|c|c|c|c|c|}
\hline \multicolumn{7}{|c|}{ Quality assessment } & \multicolumn{2}{|c|}{ No of patients } & \multicolumn{2}{|c|}{ Effect } & \multirow{2}{*}{ Quality } & \multirow{2}{*}{ Importance } \\
\hline $\begin{array}{l}\text { No of } \\
\text { studies }\end{array}$ & Design & $\begin{array}{c}\text { Risk of } \\
\text { bias }\end{array}$ & Inconsistency & Indirectness & Imprecision & $\begin{array}{c}\text { Other } \\
\text { considerations }\end{array}$ & $\begin{array}{c}\text { Contact } \\
\text { anaesthesia }\end{array}$ & \begin{tabular}{|c|} 
Topica \\
anaesthesia
\end{tabular} & $\begin{array}{l}\text { Relative } \\
(95 \% \mathrm{Cl})\end{array}$ & Absolute & & \\
\hline
\end{tabular}

Pain during the administration of the anaesthetic agent (follow-up 1 days; measured with: 5-point scale; better indicated by lower values)

\begin{tabular}{|c|c|c|c|c|c|c|c|c|c|c|}
\hline 1 & $\begin{array}{l}\text { randomised } \\
\text { trials } \\
\text { tery } \\
\text { serious }{ }^{1}\end{array}$ & $\begin{array}{l}\text { no serious } \\
1 \text { inconsistency }\end{array}$ & $\begin{array}{l}\text { no serious } \\
\text { indirectness }\end{array}$ & serious $^{2}$ & none & 25 & 25 & - & $\begin{array}{c}\text { MD } 0.04 \\
\text { higher } \\
(0.15 \\
\text { lower to } \\
0.23 \\
\text { higher })\end{array}$ & $\begin{array}{l}\text { पिमा } \\
\text { VERY } \\
\text { LOW }\end{array}$ \\
\hline
\end{tabular}

Pain during surgery (follow-up 1 days; measured with: 5-point scale; better indicated by lower values)

\begin{tabular}{|c|c|c|c|c|c|c|c|c|c|c|}
\hline 1 & $\begin{array}{l}\text { randomised } \\
\begin{array}{ll}\text { very } \\
\text { trials } & \text { serious }\end{array}\end{array}$ & $\begin{array}{l}\text { no serious } \\
1 \text { inconsistency }\end{array}$ & $\begin{array}{l}\text { no serious } \\
\text { indirectness }\end{array}$ & $\begin{array}{l}\text { no serious } \\
\text { imprecision }\end{array}$ & none & 25 & 25 & - & $\begin{array}{c}\text { MD } 1.36 \\
\text { lower } \\
(1.91 \text { to } \\
0.81 \\
\text { lower) }\end{array}$ & LOW \\
\hline \multicolumn{11}{|c|}{ Pain during inmediatly after surgery (follow-up 1 days; measured with: 5-point scale; better indicated by lower values) } \\
\hline 1 & randomised very & no serious & no serious & serious $^{2}$ & none & 25 & 25 & - & MD 0.68 & $\square \square \square \square$ \\
\hline
\end{tabular}




\begin{tabular}{|c|c|c|c|c|c|c|c|c|c|c|c|c|}
\hline & trials & serious $^{1}$ & inconsistency & indirectness & & & & & & \begin{tabular}{|c|} 
lower \\
$(1.06$ to \\
0.3 \\
lower $)$
\end{tabular} & $\begin{array}{l}\text { VERY } \\
\text { LOW }\end{array}$ & \\
\hline \multicolumn{13}{|c|}{ Pain 24 hours after surgery (copy) (follow-up 1 days; measured with: 5-point scale; better indicated by lower values) } \\
\hline 1 & $\begin{array}{l}\text { randomisec } \\
\text { trials }\end{array}$ & $\begin{array}{l}\text { dvery } \\
\text { serious }{ }^{1}\end{array}$ & $\begin{array}{l}\text { no serious } \\
\text { inconsistency }\end{array}$ & $\begin{array}{l}\text { no serious } \\
\text { indirectness }\end{array}$ & serious ${ }^{2}$ & none & 25 & 25 & - & \begin{tabular}{|c} 
MD 0.28 \\
lower \\
$(0.52$ to \\
0.04 \\
lower $)$
\end{tabular} & $\begin{array}{l}\text { पिएव } \\
\text { VERY } \\
\text { LOW }\end{array}$ & \\
\hline \multicolumn{13}{|c|}{ Total complications } \\
\hline 1 & $\begin{array}{l}\text { randomisec } \\
\text { trials }\end{array}$ & d serious ${ }^{1}$ & $\begin{array}{l}\text { no serious } \\
\text { inconsistency }\end{array}$ & $\begin{array}{l}\text { no serious } \\
\text { indirectness }\end{array}$ & serious $^{2}$ & none & $\begin{array}{c}3 / 25 \\
(12 \%)\end{array}$ & $\begin{array}{c}5 / 25 \\
(20 \%)\end{array}$ & $\begin{array}{c}\mathrm{RR} \\
0.55 \\
(0.12 \text { to } \\
2.58)\end{array}$ & \begin{tabular}{|c|c}
90 fewer \\
per 1.000 \\
(from \\
176 \\
fewer to \\
316 \\
more)
\end{tabular} & $\begin{array}{l}\text { Luप } \\
\text { LOW }\end{array}$ & CRITICAL \\
\hline
\end{tabular}

High risk of performance bias, selection and detection bias unclear. The study use not standardized pain scale with different cathegories using different categories. Data analysis: pain variable is analyzed as continuous one.

2Interval confidence $95 \%$ include potential benefit and potential harm/low number of events. 
Como medida del efecto, para las variables dicotómicas se utilizó el cociente de riesgos (CR) y para las variables continuas se usó la diferencia de medias ponderadas (DMP), ambos con sus intervalos de confianza del 95\%. Como método estadístico se utilizaron respectivamente, los métodos de Mantel-Haenszel y de la varianza inversa. Para el metanálisis se utilizó el modelo de efectos fijos, excepto cuando se encontró heterogeneidad significativa; en ese caso, se utilizó el modelo de efectos aleatorios.

\section{FOREST PLOTS}

Anestesia de contacto vs. anestesia peribulbar. Dolor durante la administración del agente anestésico

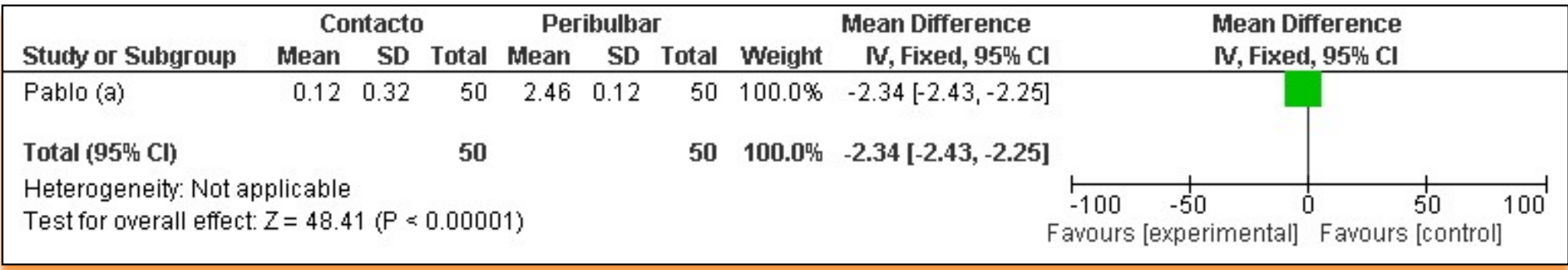

Anestesia de contacto vs. anestesia peribulbar. PIO a los 3 meses.

\begin{tabular}{|c|c|c|c|c|c|c|c|c|c|}
\hline \multirow[b]{2}{*}{ Study or Subgroup } & \multicolumn{3}{|c|}{ Experimental } & \multicolumn{3}{|c|}{ Control } & \multicolumn{2}{|r|}{ Mean Difference } & \multirow{2}{*}{$\begin{array}{l}\text { Mean Difference } \\
\mathrm{N}, \text { Fixed, } 95^{\circ} \mathrm{c} \mathrm{Cl}\end{array}$} \\
\hline & Mean & SD & Total & Mean & SD & Total & Weight & $\mathrm{N}$, Fixed, $95^{\text {听 }} \mathrm{Cl}$ & \\
\hline Pablo (a) & 16.5 & 2.65 & 50 & 16.1 & 4.65 & 50 & $100.0 \%$ & $0.40[-1.08,1.88]$ & \\
\hline Total $(95 \%$ Cl) & & & 50 & & & 50 & $100.0 \%$ & $0.40[-1.08,1.88]$ & \\
\hline $\begin{array}{l}\text { Heterogeneity: Not a } \\
\text { Test for overall effect }\end{array}$ & $\begin{array}{l}\text { plicable } \\
Z=0.53\end{array}$ & & & & & & & & 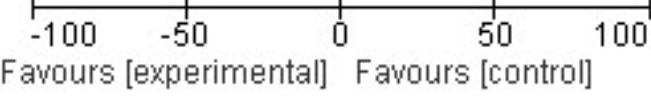 \\
\hline
\end{tabular}


Anestesia de contacto vs. anestesia peribulbar. Quimosis/hemorragia conjuntival

\begin{tabular}{|c|c|c|c|c|c|c|c|c|}
\hline \multirow[b]{2}{*}{ Study or Subgroup } & \multicolumn{2}{|c|}{ Experimental } & \multicolumn{2}{|c|}{ Control } & \multirow[b]{2}{*}{ Weight } & \multirow{2}{*}{$\begin{array}{c}\text { Odds Ratio } \\
\text { M-H, Fixed, 95\% } \mathrm{Cl} \\
\end{array}$} & \multirow{2}{*}{\multicolumn{2}{|c|}{$\begin{array}{c}\text { Odds Ratio } \\
\text { M-H, Fixed, 95\% } \mathrm{Cl}\end{array}$}} \\
\hline & Events & Total & Events & Total & & & & \\
\hline Pablo (a) & 0 & 50 & 6 & 50 & $100.0 \%$ & $0.07[0.00,1.24]$ & ] & \\
\hline Total $(95 \%$ 政 & & 50 & & 50 & $100.0 \%$ & $0.07[0.00,1.24]$ & & \\
\hline Total events & 0 & & 6 & & & & & \\
\hline \multicolumn{3}{|c|}{$\begin{array}{l}\text { Heterogeneity: Not applicable } \\
\text { Test for overall effect: } Z=1.82(P=0.07)\end{array}$} & & & & & $\begin{array}{cc}0.01 & 0.1 \\
\text { Favours [experimental] }\end{array}$ & $\begin{array}{c}10 \\
\text { Favours [control] }\end{array}$ \\
\hline
\end{tabular}

Anestesia de contacto vs. anestesia peribulbar. Otros eventos adversos (como hifema, fugas de la ampolla, cámara anterior superficial, desprendimiento coroidal o complicaciones que hayan requerido reintervención quirúrgica)

\begin{tabular}{|c|c|c|c|c|c|c|c|}
\hline \multirow[b]{2}{*}{ Study or Subgroup } & \multicolumn{2}{|c|}{ Experimental } & \multicolumn{2}{|c|}{ Control } & \multirow[b]{2}{*}{ Weight } & \multirow{2}{*}{$\begin{array}{c}\text { Odds Ratio } \\
\text { M-H, Fixed, 95吅 C }\end{array}$} & \multirow{2}{*}{ 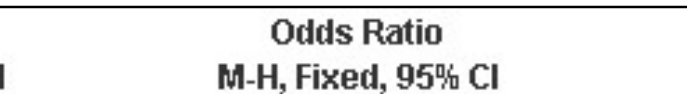 } \\
\hline & Events & Total & Events & Total & & & \\
\hline Pablo (a) & 11 & 50 & 11 & 50 & $100.0 \%$ & $1.00[0.39,2.58]$ & ]] \\
\hline Total $(95 \% \mathrm{Cl})$ & & 50 & & 50 & $100.0 \%$ & $1.00[0.39,2.58]$ & \\
\hline Total events & 11 & & 11 & & & & \\
\hline $\begin{array}{l}\text { Heterogeneity: Not a } \\
\text { Test for overall effec }\end{array}$ & $\begin{array}{l}\text { plicable } \\
Z=0.00\end{array}$ & $=1.00$ & & & & & $\begin{array}{cccc}0.01 & 0.1 & 1 & 10 \\
\text { Favours [experimental] } & \text { Favours [control] }\end{array}$ \\
\hline
\end{tabular}


4.5.5. Anestesia subtenoniana vs. anestesia retrobulbar

Question: Should Sub-Tenon's Anesthesia vs retrobulbar anesthesia be used for open angle glaucoma surgery? Bibliography:

Buys YM, Trope GE. Prospective study of sub-Tenon's versus retrobulbar anesthesia for inpatient and day-surgery trabeculectomy Ophthalmology. 1993; 100(10): 1585-9.

\begin{tabular}{|c|c|c|c|c|c|c|c|c|c|c|c|c|}
\hline \multicolumn{7}{|c|}{ Quality assessment } & \multicolumn{2}{|c|}{ No of patients } & \multicolumn{2}{|c|}{ Effect } & \multirow{2}{*}{ Quality } & \multirow{2}{*}{ Importance } \\
\hline \begin{tabular}{|l|} 
No of \\
studies
\end{tabular} & Design & $\begin{array}{c}\text { Risk of } \\
\text { bias }\end{array}$ & Inconsistency & Indirectness & Imprecision & $\begin{array}{c}\text { Other } \\
\text { considerations }\end{array}$ & \begin{tabular}{|c|} 
Sub- \\
Tenon's \\
Anesthesia
\end{tabular} & $\begin{array}{l}\text { Retrobulbar } \\
\text { anesthesia }\end{array}$ & $\begin{array}{l}\text { Relative } \\
(95 \% \mathrm{Cl})\end{array}$ & Absolute & & \\
\hline \multicolumn{13}{|c|}{ Intraoperative Disconfort (follow-up 1 days; measured with: 5 point scale; better indicated by lower values) } \\
\hline 1 & $\begin{array}{l}\text { randomised } \\
\text { trials }\end{array}$ & $\begin{array}{l}\text { very } \\
\text { serious }\end{array}$ & $\begin{array}{l}\text { no serious } \\
\text { inconsistency }\end{array}$ & $\begin{array}{l}\text { no serious } \\
\text { indirectness }\end{array}$ & serious $^{2}$ & none & 22 & 17 & - & \begin{tabular}{|c|} 
MD 0.1 \\
higher \\
$(0.6$ lower \\
to 0.4 \\
higher $)$
\end{tabular} & \begin{tabular}{|l|} 
पिएव \\
VERY \\
LOW
\end{tabular} & CRITICAL \\
\hline \multicolumn{13}{|c|}{ Post-operative disconfort -24 hoursafter surgery (follow-up 1 days; measured with: 5 point scale; better indicated by lower values) } \\
\hline 1 & $\begin{array}{l}\text { randomised } \\
\text { trials }\end{array}$ & $\begin{array}{l}\mathrm{d} \text { very } \\
\text { serious }\end{array}$ & $\begin{array}{l}\text { no serious } \\
\text { inconsistency }\end{array}$ & $\begin{array}{l}\text { no serious } \\
\text { indirectness }\end{array}$ & serious $^{2}$ & none & 22 & 17 & - & \begin{tabular}{|c|} 
MD 0.6 \\
lower (0.8 \\
lower to \\
0.6 \\
higher)
\end{tabular} & \begin{tabular}{|l|} 
पिमा \\
VERY \\
LOW
\end{tabular} & CRITICAL \\
\hline \multicolumn{13}{|c|}{ Postoperative analgesia use } \\
\hline 1 & randomised & d very & no serious & serious $^{3}$ & serious $^{2}$ & none & $7 / 22$ & $12 / 17$ & $\begin{array}{l}\mathrm{RR} \\
0.45\end{array}$ & \begin{tabular}{|l|}
388 fewer \\
per 1.000
\end{tabular} & \begin{tabular}{|l|} 
पिप्र \\
VERY
\end{tabular} & \\
\hline
\end{tabular}




\begin{tabular}{|c|c|c|c|c|c|c|c|c|c|c|c|c|}
\hline & trials & serious $^{1}$ & inconsistency & & & & $(31.8 \%)$ & $(70.6 \%)$ & \begin{tabular}{|c|}
$(0.23$ to \\
$0.89)$
\end{tabular} & \begin{tabular}{|c|} 
(from 78 \\
fewer to \\
544 \\
fewer)
\end{tabular} & LOW & \\
\hline \multicolumn{13}{|c|}{ Hemorragia subconjuntival } \\
\hline 1 & \begin{tabular}{|l|} 
randomised \\
trials
\end{tabular} & $\begin{array}{l}\text { very } \\
\text { serious }\end{array}$ & \begin{tabular}{|l|} 
no serious \\
inconsistency
\end{tabular} & \begin{tabular}{|l} 
no serious \\
indirectness
\end{tabular} & serious $^{2}$ & none & $\begin{array}{c}2 / 22 \\
(9.1 \%)\end{array}$ & $\begin{array}{c}1 / 17 \\
(5.9 \%)\end{array}$ & \begin{tabular}{|c|} 
RR \\
1.55 \\
$(0.15$ to \\
$15.66)$
\end{tabular} & \begin{tabular}{|c|}
32 more \\
per 1.000 \\
(from 50 \\
fewer to \\
862 more)
\end{tabular} & $\begin{array}{l}\text { पिपर } \\
\text { VERY } \\
\text { LOW }\end{array}$ & CRITICAL \\
\hline \multicolumn{13}{|c|}{ Retrobulbar hemorrhage } \\
\hline 1 & \begin{tabular}{|l|} 
randomised \\
trials
\end{tabular} & \begin{tabular}{|l|} 
very \\
serious
\end{tabular} & \begin{tabular}{|l|} 
no serious \\
inconsistency
\end{tabular} & $\begin{array}{l}\text { no serious } \\
\text { indirectness }\end{array}$ & serious $^{2}$ & none & $\begin{array}{l}0 / 22 \\
(0 \%)\end{array}$ & $\begin{array}{c}1 / 17 \\
(5.9 \%)\end{array}$ & \begin{tabular}{|c|} 
RR \\
0.26 \\
$(0.01$ to \\
$6.03)$
\end{tabular} & $\begin{array}{c}44 \text { fewer } \\
\text { per } 1.000 \\
\text { (from } 58 \\
\text { fewer to } \\
296 \text { more) }\end{array}$ & \begin{tabular}{|l|} 
पिमा \\
VERY \\
LOW
\end{tabular} & CRITICAL \\
\hline \multicolumn{13}{|c|}{ Hyphema } \\
\hline 1 & \begin{tabular}{|l|} 
randomised \\
trials
\end{tabular} & \begin{tabular}{|l|} 
very \\
serious
\end{tabular} & $\begin{array}{l}\text { no serious } \\
\text { inconsistency }\end{array}$ & $\begin{array}{l}\text { no serious } \\
\text { indirectness }\end{array}$ & serious $^{2}$ & none & $\begin{array}{l}0 / 22 \\
(0 \%)\end{array}$ & $\begin{array}{c}1 / 17 \\
(5.9 \%)\end{array}$ & \begin{tabular}{|c|} 
RR \\
0.26 \\
$(0.01$ to \\
$6.03)$
\end{tabular} & $\begin{array}{c}44 \text { fewer } \\
\text { per } 1.000 \\
\text { (from } 58 \\
\text { fewer to } \\
296 \text { more) }\end{array}$ & \begin{tabular}{|l|} 
पि口प \\
VERY \\
LOW
\end{tabular} & CRITICAL \\
\hline
\end{tabular}

${ }^{1}$ High risk of performance and detection bias. Allocation concealment unclear.

2 Interval confidence $95 \%$ include potential benefit and potential harm/low number of events.

${ }^{3}$ Postoperative analgesia use, indirect measure of pain after sugery. 


\section{MÉTODOS}

Como medida del efecto, para las variables dicotómicas se utilizó el cociente de riesgos (CR) y para las variables continuas se usó la diferencia de medias ponderadas (DMP), ambos con sus intervalos de confianza del 95\%. Como método estadístico se utilizaron, respectivamente, los métodos de Mantel-Haenszel y de la varianza inversa. Para el metanálisis se utilizó el modelo de efectos fijos, excepto cuando se encontró heterogeneidad significativa; en ese caso, se utilizó el modelo de efectos aleatorios.

\section{FOREST PLOTS}

Anestesia subtenoniana respecto a anestesia retrobulbar. Uso de analgesia post-operatoria

\begin{tabular}{|c|c|c|c|c|c|c|c|c|}
\hline \multirow[b]{2}{*}{ Study or Subgroup } & \multicolumn{2}{|c|}{ Experimental } & \multicolumn{2}{|c|}{ Control } & \multirow[b]{2}{*}{ Weight } & \multirow{2}{*}{$\begin{array}{c}\text { Risk Ratio } \\
\text { M-H, Fixed, 95听 Cl }\end{array}$} & \multirow{2}{*}{\multicolumn{2}{|c|}{$\begin{array}{c}\text { Risk Ratio } \\
\text { M-H, Fixed, 95\% } \mathrm{Cl}\end{array}$}} \\
\hline & Events & Total & Events & Total & & & & \\
\hline Buys 1993 & 7 & 22 & 12 & 17 & $100.0 \%$ & $0.45[0.23,0.89]$ & & \\
\hline Total $(95 \%$ Cl) & & 22 & & 17 & $100.0 \%$ & $0.45[0.23,0.89]$ & & \\
\hline Total events & 7 & & 12 & & & & & \\
\hline \multicolumn{3}{|c|}{$\begin{array}{l}\text { Heterogeneity: Not applicable } \\
\text { Test for overall effect: } Z=2.28(P=0.02)\end{array}$} & & & & & $\begin{array}{cc}0.01 & 0.1 \\
\text { Favours [experimental] }\end{array}$ & $\begin{array}{c}10 \\
10100 \\
\text { Favours [control] }\end{array}$ \\
\hline
\end{tabular}

Anestesia subtenoniana respecto a anestesia retrobulbar. Hemorragia subconjuntival

\begin{tabular}{|c|c|c|c|c|c|c|c|c|}
\hline \multirow[b]{2}{*}{ Study or Subgroup } & \multicolumn{2}{|c|}{ Experimental } & \multicolumn{2}{|c|}{ Control } & \multirow[b]{2}{*}{ Weight } & \multirow{2}{*}{$\begin{array}{c}\text { Risk Ratio } \\
\text { M-H, Fixed, 95呪 Cl }\end{array}$} & \multirow{2}{*}{\multicolumn{2}{|c|}{$\begin{array}{c}\text { Risk Ratio } \\
\text { M-H, Fixed, } 95 \% \text { Cl }\end{array}$}} \\
\hline & Events & Total & Events & Total & & & & \\
\hline Buys 1993 & 2 & 22 & 1 & 17 & $100.0 \%$ & $1.55[0.15,15.66]$ & & \\
\hline Total $(95 \% \mathrm{Cl})$ & & 22 & & 17 & $100.0 \%$ & $1.55[0.15,15.66]$ & & \\
\hline Total events & 2 & & 1 & & & & & \\
\hline \multicolumn{3}{|c|}{$\begin{array}{l}\text { Heterogeneity: Not applicable } \\
\text { Test for overall effect: } Z=0.37(P=0.71)\end{array}$} & & & & & $\begin{array}{c}0.01 \quad 0.1 \\
\text { Favours [experimental] }\end{array}$ & $1 \underbrace{10} 100$ \\
\hline
\end{tabular}




\section{Anestesia subtenoniana respecto a anestesia retrobulbar. Otros eventos adversos}

\section{Hemorragia retrobulbar}

\begin{tabular}{|c|c|c|c|c|c|c|c|c|}
\hline \multirow[b]{2}{*}{ Stucty or Subgroup } & \multicolumn{2}{|c|}{ Experimental } & \multicolumn{2}{|c|}{ Control } & \multirow[b]{2}{*}{ Weight } & \multirow{2}{*}{$\begin{array}{l}\text { Risk Ratio } \\
\text { M-H, Fixed, 95\% Cl }\end{array}$} & \multirow{2}{*}{\multicolumn{2}{|c|}{$\begin{array}{c}\text { Risk Ratio } \\
\text { M-H, Fixed, 95\% Cl }\end{array}$}} \\
\hline & Events & Total & Events & Total & & & & \\
\hline Buys 1993 & 0 & 22 & 1 & 17 & $100.0 \%$ & $0.26[0.01,6.03]$ & ] & \\
\hline Total $(95 \% \mathrm{Cl}$ ) & & 22 & & 17 & $100.0 \%$ & $0.26[0.01,6.03]$ & & \\
\hline Total events & 0 & & 1 & & & & & \\
\hline $\begin{array}{l}\text { Heterogeneity: Not a } \\
\text { Test for overall effect }\end{array}$ & $\begin{array}{l}\text { plicable } \\
Z=0.84(\end{array}$ & $=0.40$ & & & & & $\begin{array}{cc}0.01 & 0.1 \\
\text { Favours [experimental] }\end{array}$ & $\begin{array}{c}10 \\
100 \\
\text { Favours [control] }\end{array}$ \\
\hline
\end{tabular}

Hifema

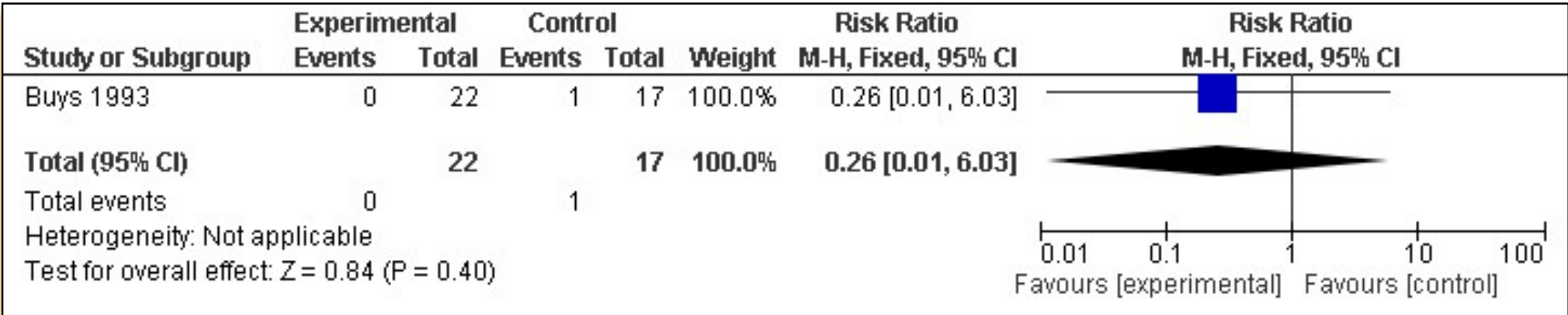


Abrasión corneal

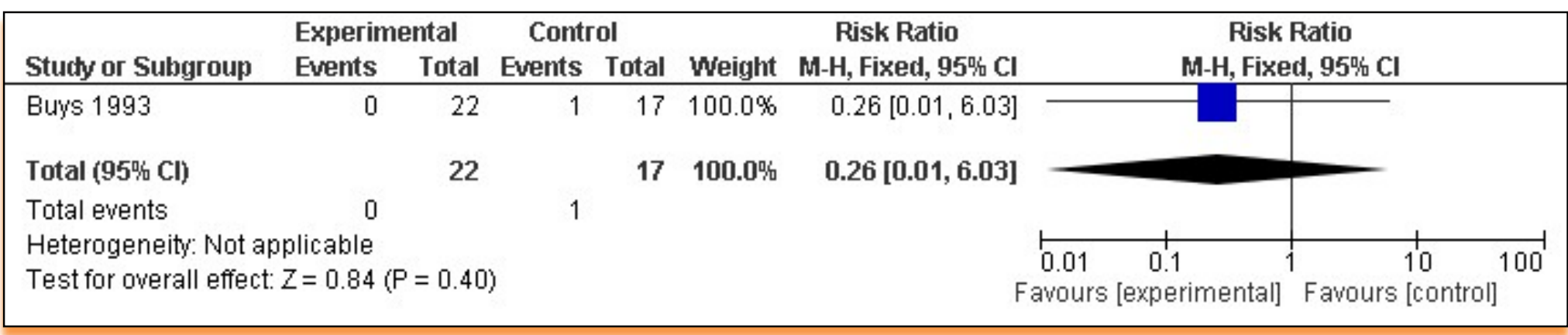




\section{Referencias}

1. Balshem $H$, Helfand $M$, Schunemann HJ, Oxman AD, Kunz R, Brozek J, et al. GRADE guidelines: 3. Rating the quality of evidence. J Clin Epidemiol. 2011;64(4):401-6.

2. Guyatt $G H$, Oxman AD, Kunz R, Vist GE, Falck-Ytter $Y$, Schunemann HJ. What is "quality of evidence" and why is it important to clinicians? BMJ. 2008; 336(7651): 995-8.

3. Guyatt GH, Oxman AD, Vist GE, Kunz R, Falck-Ytter Y, Alonso-Coello P, et al. GRADE: an emerging consensus on rating quality of evidence and strength of recommendations. BMJ. 2008;336(7650):924-6.

4. Andrews JC, Schunemann HJ, Oxman AD, Pottie K, Meerpohl JJ, Coello PA, et al. GRADE guidelines: 15. Going from evidence to recommendation-determinants of a recommendation's direction and strength. J Clin Epidemiol. 2013;66(7):726-35.

5. Alonso-Coello P, Rigau D, Sanabria AJ, Plaza V, Miravitlles M, Martínez L. Quality and strength: the GRADE system for formulating recommendations in clinical practice guidelines. Arch Bronconeumol. 2013;49(6):261-7.

6. Alonso-Coello P, Rigau D, Solà I, Martínez-García L. La formulacion de recomendaciones en salud: el sistema GRADE. Med Clin (Barc). 2013;140(8):366-73. 